Florida International University FIU Digital Commons

\title{
Evaluating Changes to Natural Variability on a Warming Globe in CMIP5 Models
}

Heather Vazquez

Florida International University, hvazq002@fiu.edu

DOI: $10.25148 /$ etd.FIDC006899

Follow this and additional works at: https://digitalcommons.fiu.edu/etd

Part of the Atmospheric Sciences Commons, Climate Commons, and the Meteorology Commons

\section{Recommended Citation}

Vazquez, Heather, "Evaluating Changes to Natural Variability on a Warming Globe in CMIP5 Models" (2018). FIU Electronic Theses and Dissertations. 3737.

https://digitalcommons.fiu.edu/etd/3737 


\title{
FLORIDA INTERNATIONAL UNIVERSITY
}

Miami, Florida

\section{EVALUATING CHANGES TO NATURAL VARIABILITY ON A WARMING GLOBE IN CMIP5 MODELS}

\author{
A dissertation submitted in partial fulfillment of \\ the requirements for the degree of \\ DOCTOR OF PHILOSOPHY \\ in \\ GEOSCIENCES \\ by \\ Heather Vazquez
}

2018 
To: $\quad$ Dean Michel R. Heithaus

College of Arts, Sciences and Education

This dissertation, written by Heather Vazquez, and entitled Evaluating Changes to Natural Variability on a Warming Globe in CMIP5 Models, having been approved in respect to style and intellectual content, is referred to you for judgment.

We have read this dissertation and recommend that it be approved.

Ben Kirtman

Joel Trexler

Hugh Willoughby

Ping Zhu

Robert Burgman, Major Professor

Date of Defense: June 22, 2018

The dissertation of Heather Vazquez is approved.

Dean Michael R. Heithaus

College of Arts, Science and Education

Andres G. Gil

Vice President for Research and Economic Development and Dean of the University Graduate School

Florida International University, 2018 
(C) Copyright 2018 by Heather Vazquez

All rights reserved. 


\section{ACKNOWLEDGMENTS}

First and foremost, I would like to give my sincerest thanks to my $\mathrm{PhD}$ advisor Dr. Robert Burgman for his continuous support and guidance over the last 5 years. I consider myself incredibly blessed to have had the opportunity to work with him throughout my academic journey. His invaluable support, guidance, and encouragement have helped shape me into the scientist I am today. I am also grateful for my committee members, Drs. Hugh Willoughby, Benjamin Kirtman, Ping Zhu, and Joel Trexler who have shared their immense knowledge and insightful comments throughout the years, helping improve the quality of my work and propel my understanding of science and other topics of interest.

I would also like to thank my former and fellow graduate students from FIU, Dr. Kathleen Fallon, Israel Gonzalez, Javiera Hernandez, Jeremy Katz, and Dr. Yongxian Pei. Their friendship has been invaluable and has provided many hours of much needed laughter throughout this academic adventure. Not to mention their encouragement and insightfulness in all things academic, weather and coding. There is no doubt I have gained remarkable lifelong colleagues.

Thank you to my amazing family, (immediate and extended), my wonderful future husband Andrew, soul sister Dianjeni, and Sunday Walker crew for their love and tremendous support throughout my time as a professional student. The many pep talks and "pull yourself together" moments allowed me to conquer my fears and get it done. Last and certainly not least, I must thank God for his undeniable favor throughout each step in this journey. His unending grace has allowed me to successfully complete this work and gave me the courage to pursue what seemed like the impossible. (Joshua 1:5-9). 


\title{
ABSTRACT OF THE DISSERTATION \\ EVALUATING CHANGES TO NATURAL VARIABILITY ON A WARMING \\ GLOBE IN CMIP5 MODELS
}

\author{
by \\ Heather Vazquez \\ Florida International University, 2018 \\ Miami, Florida

\section{Professor Robert Burgman, Major Professor}

Global mean surface temperatures (GMST) warmed in the early 20th century, experienced a mid-century lull, and warmed again steadily until 1997. Observations at the turn of the 21 st century have revealed another period of quiescent warming of GMSTs from 1998 to 2012, thus prompting the notion of a global warming "hiatus". The warming hiatus occurred concurrently with steadily increasing atmospheric greenhouse gas concentrations, sea level rise, and retreating arctic sea ice. The occurrence of the warming hiatus suggests that natural variability continues to be a sizable contributor to modern climate change and implies that energy is rearranged or changed within the climate system. Much of the scientific research conducted over the last decade has attempted to identify which modes of natural variability may be contributing to the GMST signal in the presence of anthropogenic warming. Many of these studies concluded that natural variability, operating in the global oceans were the largest contributors to GMST. What remains unclear is how oceanic variability and its contribution to GMST may change on a warmer globe as greenhouse gas concentrations continue to rise. 
Our research includes diagnostic analyses of the available observational surface temperature estimates and novel state-of-the-art climate model experiments from the fifth phase of the Coupled Model Intercomparison Project (CMIP5). Our analyses seek to understand how the natural modes of variability within the ocean will change under different warming scenarios. Utilizing simulations forced with observed pre-industrial and historical greenhouse gas emissions in combination with several future warming simulations, we quantify the probability of similar "hiatus-like" periods occurring on a warmer globe. To that end employ various metrics and detrending techniques including EOF decomposition, running climatologies, along with linear and nonlinear trends to elucidate how natural variability changes over time. We also examine the changing influence of natural modes of variability with respect to the anthropogenic radiative forcing over different regions on the globe. Results suggest that natural variability for much of the global oceans decreases as the radiative forcing increases in the future warming scenarios. 


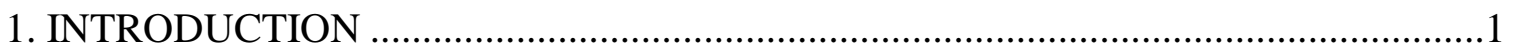

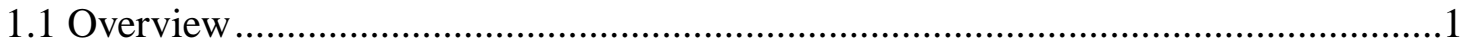

1.2 Motivation................................................................................................

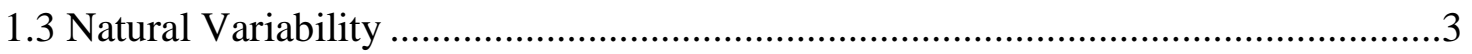

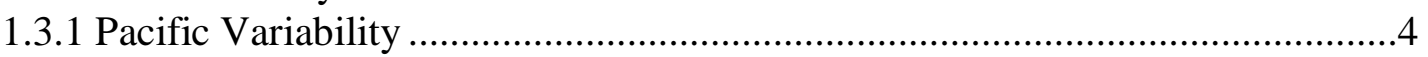

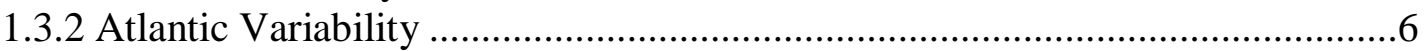

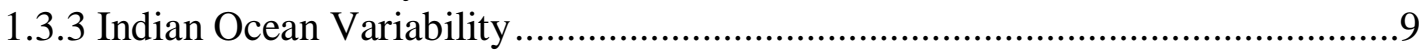

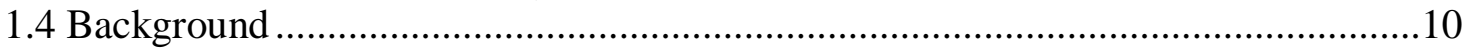

1.5 Hypothesis..................................................................................................

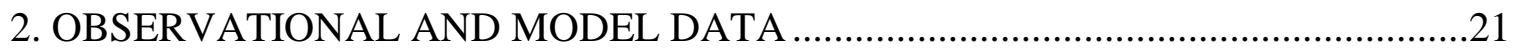

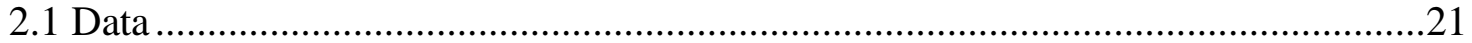

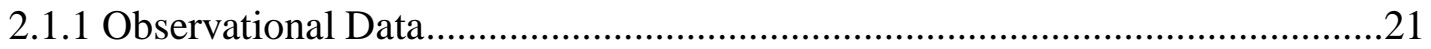

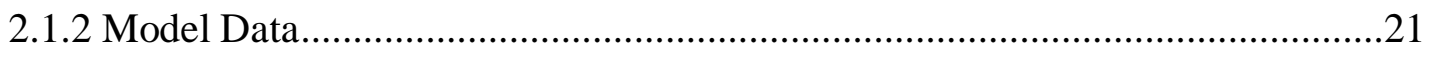

3. QUATIFYING THE PROBABILITY OF REDUCED PERIODS OF WARMING....26

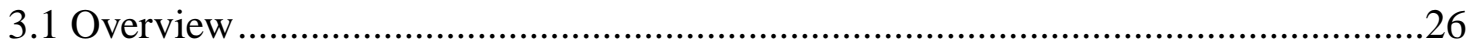

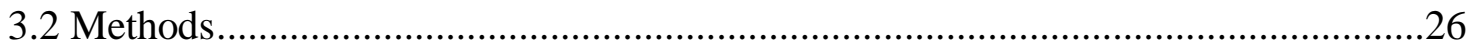

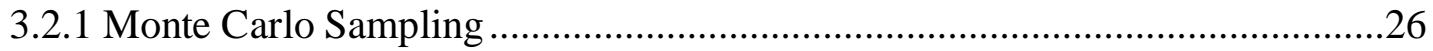

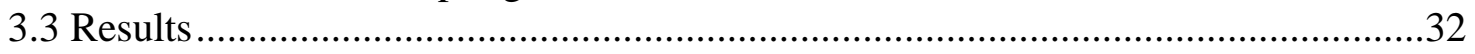

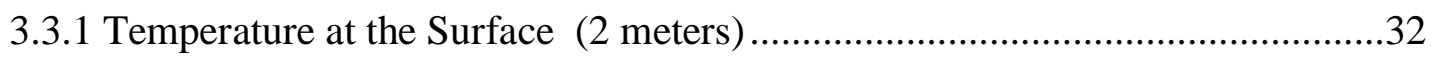

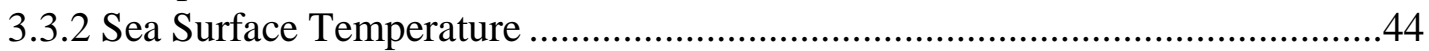

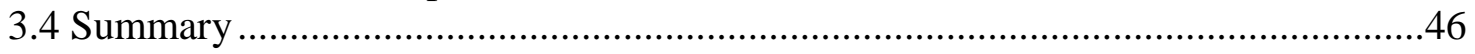

4. RESPONSE OF VARIANCE IN THE SEA SURFACE TEMPERATURE AS A RESULT OF INCREASES IN RADIATIVE FORCING ……....................................70

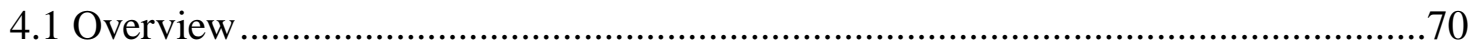

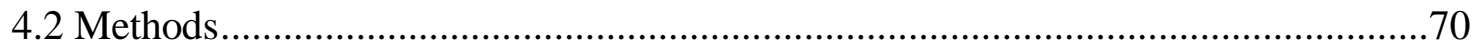

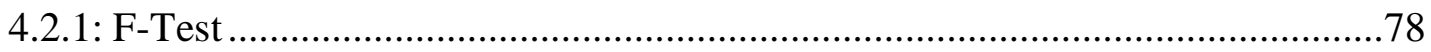

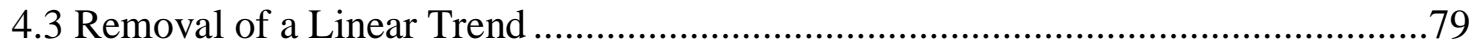

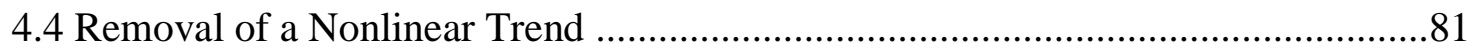

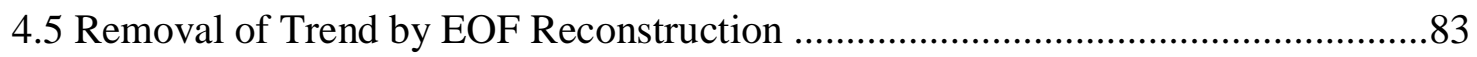

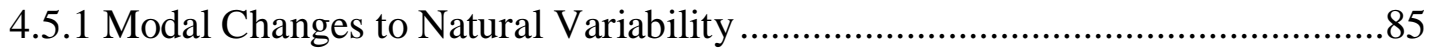

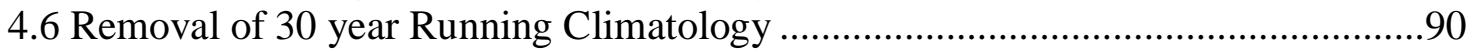

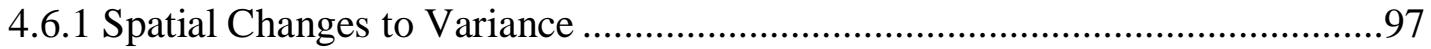

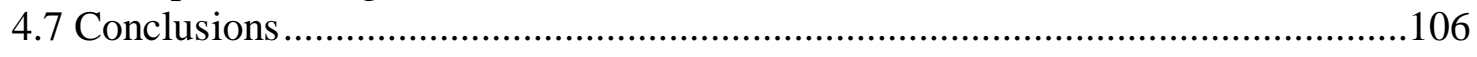

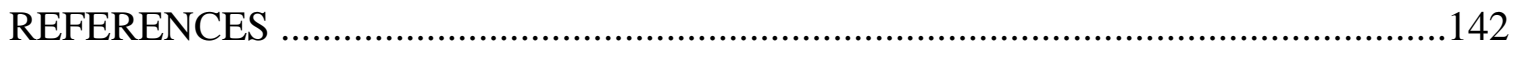

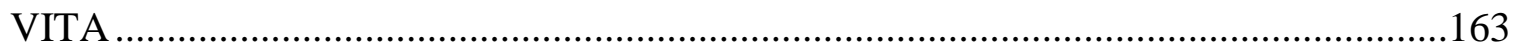




\section{LIST OF FIGURES}

FIGURE

PAGE

Figure 1.1: Taken from the IPCC report (2013), this figure is the observed global, annually averaged combined land and ocean surface temperature anomaly $\left({ }^{\circ} \mathrm{C}\right)$ between 1850-2012.

Figure 2.1: Time series of global mean surface temperature anomalies for 18 CMIP5 models. Shown are the time series' for the individual models under the Historical experiment (grey) between 1861-2005 and the four future warming experiments RCP2.6 (dark blue), RCP4.5 (red), RCP6.0 (orange), RCP8.5 (dark green) from 20062100. Also depicted are the multi-model averages for each experiment (thick lines).......24

Figure 3.1: Histogram of the Monte Carlo sampling 10,000 times over 15-year trends from the observed HadCRUT4v global mean surface temperature time series from 1850-2017. The red line depicts the IPCC calculated trend over the hiatus period (1998-2012) of $0.05^{\circ} \mathrm{C}$ per decade.

Figure 3.2: Observed annually averaged HadCRUT4 global mean surface temperature anomaly (black line) with the linearly detrended HadCRUT4 time series (blue line) and the nonlinearly detrended HadCRUT4 time series (orange line) from 1850-2017.....51

Figure 3.3: Autocorrelation Plot of the first 100 lags for a) observed GMST of HadCRUTv4 b) linearly detrended HadCRUT4v GMST between 1850-2017.

Figure 3.4: Time series of the linearly detrended annually averaged HadCRUT4 global mean surface temperature anomaly from 1850-2017 (blue curve) and one iteration from the AR1 modeled HadCRUT4 time series (chosen by the author; orange curve)

Figure 3.5: Box and whisker plots of the Monte Carlo sampled 15-year trends over 10,000 times from the HadCRUT4 GMST between 1850-2017 and the last 100 years for 18 CMIP5 models for the PiControl experiment which experience forcing at 280 ppm throughout the entire simulation. The green line denotes the IPCC calculated trend of $0.05^{\circ} \mathrm{C}$ per decade over the hiatus period from 1998-2012.

Figure 3.6: Box and whisker plots of the Monte Carlo sampled 15-year trends over 10,000 times from the HadCRUT4 GMST between 1850-2017 and 18 CMIP5 models for the Historical experiment from 1861-2005. The green line denotes the IPCC calculated trend of $0.05^{\circ} \mathrm{C}$ per decade over the hiatus period from 1998-2012

Figure 3.7: Box and whisker plots of the Monte Carlo sampled 15-year trends over 10,000 times from the AR1 simulated HadCRUT4 data between 1850-2017 and 18 AR1 simulated Historical models from 1861-2005. The green line denotes the 
detrended IPCC calculated trend of $0.002^{\circ} \mathrm{C}$ per decade over the hiatus period from $1998-2012$

Figure 3.8: Box and whisker plots of the Monte Carlo sampled 15-year trends over 10,000 times for 18 CMIP5 models under the RCP2.6 experiment (2006-2100) in red and the RCP8.5 experiment (2006-2100) in blue. The green line denotes the IPCC calculated trend of $0.05^{\circ} \mathrm{C}$ per decade over the hiatus period from 1998-2012

Figure 3.9: Box and whisker plots of the Monte Carlo sampled 15-year trends over 10,000 times for 18 linearly detrended CMIP5 models under the RCP2.6 experiment (2006-2100) in red and the RCP8.5 experiment (2006-2100) in blue. The green line denotes the linearly detrended IPCC calculated trend of $0.002^{\circ} \mathrm{C}$ per decade over the hiatus period from 1998-2012

Figure 3.10: Box and whisker plots of the Monte Carlo sampled 15-year trends over 10,000 times for 18 linearly detrended models under the RCP2.6 experiment. Red boxes are the Monte Carlo sampled 15-year trends calculated over the modeled years, 2006-2050 in each model member and blue boxes are the 15-year trends sampled over the modeled years 2051-2100 in each member. The green line denotes the linearly detrended IPCC calculated trend of $0.002^{\circ} \mathrm{C}$ per decade over the hiatus period from 1998-2012

Figure 3.11: Box and whisker plots of the Monte Carlo sampled 15-year trends over 10,000 times for 18 linearly detrended models under the RCP8.5 experiment. Red boxes are the Monte Carlo sampled 15-year trends calculated over the modeled years, 2006-2050 in each model member and blue boxes are the 15-year trends sampled over the modeled years 2051-2100 in each member. The green line denotes the linearly detrended IPCC calculated trend of $0.002^{\circ} \mathrm{C}$ per decade over the hiatus period from 1998-2012

Figure 3.12: Scatter plots of the model variance versus interquartile range of the Monte Carlo sampled 15-year trends over 10,000 times before linearly detrending for 18 model members under the a) PiControl (last 100 years in each model), b) Historical (1861-2005), d) RCP2.6 (2006-2100) and d) RCP8.5 (2006-2100) experiment

Figure 3.13: Distribution of the Monte Carlo sampled 15-year trends over 10,000 times over the a) observed HadCRUT4 time series (1850-2017) and for the multimodel GMST time series concatenated for the full record of each model member for experiments b) PiControl, c) Historical, d) RCP2.6, e) RCP4.5, f) RCP6.0, g) RCP8.5. The red line depicts the trend calculated over the recent hiatus (1998-2012) in HadCRUT4. Combining the 18-member ensemble gives us 1800 simulated years to sample over the last 100 years in each PiControl experiment, 2610 modeled years in the Historical experiment from 1861-2005, and 1710 modeled years for all four RCP experiments between 2006-2100. 
Figure 3.14: Distribution of the Monte Carlo sampled 15-year trends over 10,000 times over the first 50 years (1900-1950) for the a) observed HadCRUT4 time series and for the multi-model GMST time series concatenated with the first 50 years of each model member for CMIP5 experiments b) PiControl, c) Historical, d) RCP2.6, e) RCP4.5, f) RCP6.0, g) RCP8.5. The red line depicts the trend calculated over the recent hiatus (1998-2012) in HadCRUT4. Combining the 18-member ensemble gives us 900 simulated years to sample over the first years in each PiControl experiment and the Historical experiment from 1900-1950, and 792 modeled years for all four RCP experiments between 2006-2050

Figure 3.15: Distribution of the Monte Carlo sampled 15-year trends over 10,000 times over the last 50 years (1951-2017) over the a) observed HadCRUT4 time series and for the multi-model GMST time series concatenated with the last 50 years of each model member for CMIP5 experiments b) PiControl, c) Historical, d) RCP2.6, e) RCP4.5, f) RCP6.0, g) RCP8.5. The red line depicts the trend calculated over the recent hiatus (1998-2012) in HadCRUT4. Combining the 18-member ensemble gives us 900 simulated years to sample over the last 50 years in each PiControl experiment, 972 simulated years for the Historical experiment from 1951-2005, and 900 modeled years for all four RCP experiments between 2051-2100

Figure 3.16: Box and whiskers plot of the Monte Carlo sampled 15-year trends over 10,000 times for the multi-model linearly detrended time series concatenated using the full GMST for the PiControl, Historical, RCP2.6, RCP4.5, RCP6.0, and RCP8.5. Combining the 18-member ensemble over the full record results in 1800 simulated years to sample over the last 100 years in each PiControl experiment, 2610 modeled years in the Historical experiment from 1861-2005, and 1710 modeled years for all four RCP experiments between 2006-2100Type chapter title (level 1)

Figure 3.17: Box and whisker plots of the Monte Carlo sampled 15-year trends over 10,000 times from the multi-model linearly detrended time series concatenated with a) the first 50 years of each ensemble member and b) the last 50 years of each ensemble member for the PiControl, Historical, RCP2.6, RCP4.5, RCP6.0, and RCP8.5 experiments. Combining the 18 -member ensemble over the first years produces 900 simulated years to sample in each PiControl experiment, 972 simulated years for the Historical experiment from 1900-1950, and 900 modeled years for all four RCP experiments between 2006-2050. While combining over the last half of each model member produces 900 simulated years to sample over the last 50 years in each PiControl experiment, 972 simulated years for the Historical experiment from 1951-2005, and 900 modeled years for all four RCP experiments between 2051-2100 ...66

Figure 3.18: Normalized histogram of the Monte Carlo sampled 15-year trends over 10,000 times for the linearly detrended full record of the multi-model time series for the PiControl, Historical, RCP2.6, RCP4.5, RCP6.0, and RCP8.5. Combining the 18member ensemble results in 1800 simulated years to sample over the last 100 years in each PiControl experiment, 2610 simulated years in the Historical experiment from 
1861-2005, and 1710 modeled years for all four RCP experiments between 2006-2100. The black line depicts the trend calculated over the recent hiatus (1998-2012) in HadCRUT4

Figure 3.19: Box and whiskers plot of the Monte Carlo sampled 15-year trends over 10,000 times for the multi-model linearly detrended time series concatenated using the full global mean sea surface temperature for the PiControl, Historical, RCP2.6, RCP4.5, RCP6.0, and RCP8.5. Combining the 18-member ensemble over the full record results in 1800 simulated years to sample over the last 100 years in each PiControl experiment, 2610 modeled years in the Historical experiment from 18612005, and 1710 modeled years for all four RCP experiments between 2006-2100

Figure 3.20: Box and whisker plots of the Monte Carlo sampled 15-year trends over 10,000 times from the multi-model linearly detrended global mean sea surface temperature time series concatenated with a) the first 50 years of each ensemble member and b) the last 50 years of each ensemble member for the PiControl, Historical, RCP2.6, RCP4.5, RCP6.0, and RCP8.5 experiments. Combining the 18 -member ensemble over the first 50 years produces 900 simulated years to sample in each PiControl experiment, 972 simulated years for the Historical experiment from 1900-1950, and 900 modeled years for all four RCP experiments between 2006-2050. While combining over the last half of each model member produces 900 simulated years to sample over the last 50 years in each PiControl experiment, 972 simulated years for the Historical experiment from 1951-2005, and 900 modeled years for all four RCP experiments between 2051-2100

Figure 4.1:Principle Components for the first 20 EOFs in GISS-E2-H under the Historical experiment (1861-2005). The trend is observed in the leading mode (EOF 1)

Figure 4.2: The (left) three leading EOFs with variance explained (in percent) of ERSSTv4 with their associated PCs of annual mean SST over 1901-2017 and (right) the three leading EOFs of ERSSTv4 after the removal of the first EOF and associated PCs

Figure 4.3: Observed annually averaged ERSSTv4 global mean sea surface temperature anomaly (black line) with the linearly detrended ERSSTv4 time series (blue line), nonlinearly detrended ERSSTv4 time series using a least-squares quadratic trend (orange line), removal of the EOF containing the largest trend (yellow line), and removing a 30-year running climatology (purple line) from 1854-2017.....

Figure 4.4: Box and whisker plots of the annually averaged multi-model ensemble GMSST before any detrending method is applied. The multi-model ensembleconsists of 19 ensemble members each detrended from the last 100 years in the PiControl run, from 1861-2005 in the Historical run, and from 2006-2100 in RCP2.6, RCP4.5, 
RCP6.0, RCP8.5. The multi-model ensemble includes a total of 1800 years for the PiControl run, 2610 years in the Historical and 1710 years for all warming scenarios.....

Figure 4.5: Box and whisker plots of the annually averaged multi-model ensemble GMSST after removing a least-squares linear trend for each individual ensemble member before concatenating. The multi-model ensemble consists of 19 ensemble members each detrended from the last 100 years in the PiControl run, from 1861-2005 in the Historical run, and from 2006-2100 in RCP2.6, RCP4.5, RCP6.0, RCP8.5. The multi-model ensemble includes a total of 1800 years for the PiControl run, 2610 years in the Historical and 1710 years for all warming scenarios

Figure 4.6: Box and whisker plots of the annually averaged multi-model ensemble GMSST after removing a least-squares quadratic trend for each individual ensemble member before concatenating. The multi-model ensemble consists of 19 ensemble members each detrended from the last 100 years in the PiControl run, from 1861-2005 in the Historical run, and from 2006-2100 in RCP2.6, RCP4.5, RCP6.0, RCP8.5. The multi-model ensemble includes a total of 1800 years for the PiControl run, 2610 years in the Historical and 1710 years for all warming scenarios

Figure 4.7: Box and whisker plots of the Monte Carlo sampled 15-year trends over the multi-model ensemble for the last 100 years in each PiControl model (1800 total simulated years), Historical from 1861-2005 (2610 years), and from 2006-2100 for RCP2.6, RCP4.5, RCP6.0, an RCP8.5 (1710 years for all warming scenarios) after removing a least squares quadratic trend from the individual ensemble members before concatenation

Figure 4.8: Box and whisker plots of the annually averaged multi-model ensemble GMSST alongside the multi-model GMSST from the EOF reconstruction with the largest trend removed. Both multi-model ensembles consist of 19 ensemble members each from the last 100 years in the PiControl run, from 1861-2005 in the Historical run, and from 2006-2100 in RCP2.6, RCP4.5, RCP6.0, and RCP8.5. The multi-model ensembles include a total of 1800 years for the PiControl run, 2610 years in the Historical and 1710 years for all warming scenarios

Figure 4.9: Box and whisker plots of the annually averaged multi-model GMSST from EOF reconstruction after the EOF with the largest trend is removed for the individual members before concatenating. The multi-model ensemble consists of 19 ensemble members each detrended from the last 100 years in the PiControl run, from 1861-2005 in the Historical run, and from 2006-2100 in RCP2.6, RCP4.5, RCP6.0, RCP8.5. The multi-model ensemble includes a total of 1800 years for the PiControl run, 2610 years in the Historical and 1710 years for all warming scenarios ..120

Figure 4.10: Taylor Diagram where each number on the diagram corresponds to an ensemble member and the colors denote the CMIP5 experiment the ensemble 
member is under. The radial distance from the origin to model member represents the ratio of the CMIP5 experiment to the observed ERSSTv4 standard deviation. The standard deviation is calculated using the explained variance for the respective EOF modes being compared. The azimuthal positions give the weighted centered pattern correlation for the CMIP5 first two EOF modes compared to the observed ERSSTv4 first two EOF modes

Figure 4.11: Taylor Diagram where each number on the diagram corresponds to an ensemble member and the colors denote the CMIP5 experiment the ensemble member is under. The radial distance from the origin to model member represents the ratio of the CMIP5 experiment to the observed ERSSTv4 standard deviation. The standard deviation is calculated using the global variance for the respective EOF modes being compared. The azimuthal positions give the weighted centered pattern correlation for the CMIP5 first two EOF modes compared to the observed ERSSTv4 first two EOF modes

Figure 4.12: Spatial pattern of the second EOF for ERSSTv4 for the time period between 1850-2017 compared to the spatial pattern of the second EOF for 19 ensemble members under RCP8.5 radiative forcing from 2006-2100

Figure 4.13: Scatter plot of linear trend per decade calculated using the trend found in the first EOF vs the global variance calculated in the second EOF for a) the individual model members and $b$ ) the multi-model ensemble average

Figure 4.14: Taken from the IPCC report (2013); Standard deviation in CMIP5 multimodel ensembles of sea surface temperature variability over the eastern equatorial Pacific Ocean (Nino3 region: $5^{\circ} \mathrm{S}-5^{\circ} \mathrm{N}, 150^{\circ} \mathrm{W}-90^{\circ} \mathrm{W}$ ), a measure of El Nino amplitude, for the pre-industrial (PI) control and 20th century (20C) simulations, and 21 st century projections using RCP4.5 and RCP8.5. Thirty-one models are used for the ensemble average. Open circles indicate multi-model ensemble means, and $t$ he red cross symbol is the observed standard deviation for January 1870 - December 2011 obtained from HadISSTv1. The linear trend and climatological mean of seasonal cycle have been removed. Box-whisker plots show the 16th, 25th, 50th, 75th, and 84th percentiles

Figure 4.15: Box and whisker plots of the annually averaged multi-model ensemble GMSST with 30-year running climatology removed. The multi-model ensemble consists of 19 ensemble members each detrended from the last 100 years in the PiControl run, from 1861-2005 in the Historical run, and from 2006-2100 in RCP2.6, RCP4.5, RCP6.0, RCP8.5. The multi-model ensemble includes a total of 1260 years for the PiControl run, 2070 years in the Historical and 1170 years for all warming scenarios. 
Figure 4.16: Box and whisker plots of the annually averaged multi-model ensemble GMSST with 30 year running climatology removed for the PiControl (1260 years), Historical (2070 years), a Historical greenhouse gas only run (1885 year), and RCP2.6, RCP4.5, RCP6.0, RCP8.5 (1170 years for all warming scenarios)

Figure 4.17: Scatter plot of linear trend per decade vs variance of each model after removing 30 year running mean for the individual model members on the left and the multi-model ensemble average on the right

Figure 4.18: Scatter plot of the multi-model ensemble average linear trend per decade calculated from the raw SST data in the a) Atlantic Ocean b) Pacific Ocean c) Indian Ocean d) Southern Ocean e) Artic Ocean vs variance calculated in each basin for 19 models for PiControl, Historical, RCP2.6, RCP4.5, RCP6.0, and RCP8.5

Figure 4.19: Scatter plot of the multi-model ensemble average linear trend per decade vs variance for 19 models for PiControl, Historical, RCP2.6, RCP4.5, RCP6.0, and RCP8.5 by a) latitude $5 \mathrm{~S}$ to $5 \mathrm{~N} \mathrm{~b}$ ) latitude $10 \mathrm{~S}$ to $10 \mathrm{~N} \mathrm{c}$ ) latitude $15 \mathrm{~S}$ to $15 \mathrm{~N}$ d) latitude $30 \mathrm{~S}$ to $30 \mathrm{~N}$ e) latitude $45 \mathrm{~S}$ to $45 \mathrm{~N}$ f) latitude $60 \mathrm{~S}$ to $60 \mathrm{~N}$.

Figure 4.20: Scatter plot of ensemble variance vs sea surface temperature gradient for 19 models for PiControl, Historical, RCP2.6, RCP4.5, RCP6.0, and RCP8.5 by latitude a) $0-20 \mathrm{~N} \mathrm{~b}) 0-45 \mathrm{~N} \mathrm{c}) 0-60 \mathrm{~N} \mathrm{~d}) 0: 80 \mathrm{~N} \mathrm{e}) 20 \mathrm{~N}-45 \mathrm{~N} \mathrm{f}) 20 \mathrm{~N}-60 \mathrm{~N}$

Figure 4.21: Scatter plot of ensemble variance vs sea surface temperature gradient for 19 models for PiControl, Historical, RCP2.6, RCP4.5, RCP6.0, and RCP8.5 by latitude a) $0-20 \mathrm{~S}$ b) $0-45 \mathrm{~S}$ c) $0-50 \mathrm{~S}$ d) $0: 60 \mathrm{~S}$ e) $20 \mathrm{~S}-45 \mathrm{~S} \mathrm{f}) 20 \mathrm{~S}-60 \mathrm{~S}$

Figure 4.22: Ratio of two variances of SSTA to the Historical run in the Arctic between the a) RCP26 simulation, b) RCP45 simulation, c) RCP60 simulation, d) RCP85 simulation

Figure 4.23: Ratio of two variances of SSTA to the Historical run in the Antarctic between the a) RCP26 simulation, b) RCP45 simulation, c) RCP60 simulation, d) RCP85 simulation

Figure 4.24: Ratio of two variances of SSTA to the Historical run from $60 \mathrm{~S}-60 \mathrm{~N}$ in the a) RCP26 simulation, b) RCP45 simulation, c) RCP60 simulation, d) RCP85 simulation. Blue shading denotes decreases in variance and Red indicated increases in variance

Figure 4.25: Variance difference by RCP scenario from Historical scenario for CCSM4 (contoured). Red denotes an increase in variance and Blue denotes a decrease in variance. Hatching indicates statistically significant areas at $90 \%$ significance level 
Figure 4.26: Percentage of ensemble members predicting an increase in variance that is statistically significant $(\mathrm{p}<0.10)$ from a) RCP26-Historical b) RCP45Historical, c) RCP60-Historical, and d) RCP85-Historical

Figure 4.27: Similar to Figure 4.25 but denoting the percent of ensemble members predicting a decrease in variance that is statistically significant $(\mathrm{p}<0.10)$ from a) RCP26-Historical b) RCP45-Historical, c) RCP60-Historical, and d) RCP85-Historical....

Figure 4.28: Variance difference for CCSM4 on the left column for a) RCP26Historical c) RCP45-Historical, e) RCP60-Historical, and g) RCP85-Historical and CESM1-CAM5 on the right column for b) RCP26-Historical d) RCP45Historical, f) RCP60-Historical, and h) RCP85-Historical. Blue indicates decreases in variance and Red denotes increases in variance

Figure 4.29: Variance difference for GFDL-CM3 is on the left column for a) RCP26Historical d) RCP45-Historical, g) RCP60-Historical, and j) RCP85-Historical. The middle column is the variance difference for GFDL-ESM2G for b) RCP26-Historical, e) RCP45-Historical, h) RCP60-Historical, and k) RCP85-Historical and GFDLESM2M on the right column for c) RCP26-Historical f) RCP45-Historical, i) RCP60Historical, and 1) RCP85-Historical. Blue indicates decreases in variance and Red denotes increases in variance 


\section{LIST OF ABBREVATIONS AND ACRONYMS}

\begin{tabular}{|c|c|}
\hline AMO & Atlantic Multidecadal Oscillation \\
\hline AMOC & Atlantic Meridional Overturning Circulation \\
\hline AMV & Atlantic Multidecadal Variability \\
\hline AR1 & First Order Autoregressive Process \\
\hline CMIP3 & Third Phase of the Coupled Model Intercomparison Project \\
\hline CMIP5 & Fifth Phase of the Coupled Model Intercomparison Project \\
\hline ENSO & El Niño Southern Oscillation \\
\hline $\mathrm{EOF}$ & Empirical Orthogonal Function \\
\hline ESM & Earth System Model \\
\hline GCM & Global Climate Model \\
\hline GFDL & Geophysical Fluid Dynamics Laboratory \\
\hline GHG & Greenhouse Gases \\
\hline GMST & Global Mean Surface Temperature \\
\hline GMST & Global Mean Surface Temperature Anomaly \\
\hline GMSST & Global Mean Sea Surface Temperature \\
\hline ICOADS & International Comprehensive Ocean-Atmosphere \\
\hline IOD & Indian Ocean Dipole \\
\hline IPCC & Intergovernmental Panel on Climate Change \\
\hline IQR & Interquartile Range \\
\hline ITCZ & Intertropical Convergence Zone \\
\hline NOAA & National Oceanic and Atmospheric Association \\
\hline
\end{tabular}




$\begin{array}{ll}\text { NCAR } & \text { National Center for Atmospheric Research } \\ \text { PC } & \text { Principal Component } \\ \text { PDO } & \text { Pacific Decadal Oscillation } \\ \text { PDV } & \text { Pacific Decadal Variability } \\ \text { PiControl } & \text { Preindustrial Control Run } \\ \text { RCP } & \text { Representative Concentration Pathways } \\ \text { SLP } & \text { Sea Level Pressure } \\ \text { SST } & \text { Sea Surface Temperature } \\ \text { SSTA } & \text { Sea Surface Temperature Anomaly } \\ \text { TAS } & \text { Air Temperature at the Surface (2 meters) }\end{array}$




\section{CHAPTER 1: INTRODUCTION}

\subsection{Overview}

This dissertation focuses on better understanding the likelihood of periods of reduced warming superimposed on long term warming in the future and how natural variability in the climate system will change on a warmer globe and the mechanisms and modes affected by changes to natural variability. The first section of this chapter explains the motivation for this study. Section 2 gives a brief review of several understood modes of natural variability followed by a section detailing the background and motivation of this study and the structure of this dissertation.

\subsection{Motivation}

Climate change has risen in recent decades to be the top environmental threat for future generations. By definition, climate is considered to be the long-term average of daily weather variations and conditions over several decades. The climate system of the Earth is comprised of oceans, land surface, an atmosphere, and a cryosphere. Manifestations of climate change have been observed by an increase in global land and sea surface temperatures (SST), increased water vapor in the lower troposphere, decreases in snow and ice cover, and through a steady rise in sea level by thermal expansion as a result from warming ocean temperatures and glacial ice melt, to name a few (IPCC, 2007). While there is evidence modern climate change is influenced by anthropogenic factors, natural variability still plays an important role in the variability of 
the global mean surface temperature (GMST) (Meehl et al, 2011; Trenberth and Fasullo, 2013; Kosaka and Xie, 2013).

Periods of accelerated and stagnant warming have been observed over the last century in the GMST (Figure 1.1). These periods of reduced warming are concurrent with steadily increasing greenhouse gas concentrations (GHG), sea level rise, and retreating sea ice (IPCC, 2007). In Figure 1.1, the most recent period of accelerated warming can be observed during the mid-1970s, where GMSTs climbed steadily until an unexpected period of stagnant warming was observed at the turn of the $21^{\text {st }}$ century. This period of reduced warming was known as the "Hiatus" period. Further examination of the GMST record reveals there was a similar period of stagnant warming from the mid-1940s until the mid-1970s. The climate is expected to warm over the next century (IPCC, 2013) and is common for decadal periods with little to no positive warming trend to be superimposed on centennial scale warming (Easterling and Wehner, 2009). These periods of reduced and accelerated warming in the GMST further suggest natural variability is a sizable contributor to modern climate change.

Much of modern climate change is natural resulting from both internal processes and external forcing mechanisms. External forcing mechanisms are not influenced by the climate system. Instead, these mechanisms subject the climate system to changes. They include fluctuations in the solar cycle, variations in orbital and axial parameters of the Earth, and volcanic eruptions. One example is the eruption of Mount Pinatubo in 1991. The eruption was captured by modern satellites and observing systems. Vast amounts of aerosols and gases were released into the stratosphere, significantly lowering global surface temperatures for several years (IPCC 2013). 
Internal forcing mechanisms include oceanic and atmospheric modes of variability within the climate system. These modes can be strongly coupled, resulting in a complex relationship between forcings and responses. For example, the significantly large heat capacity of the ocean causes a delay to thermal forcing. Thus, geographic variations in temperature exist as a response. In spite of this coupling, the climate system is extremely chaotic and exceedingly sensitive to change. Therefore, a slight alteration to an aspect of the system in a particular location will affect a different parameter (e.g., precipitation, temperature, etc.) in another location. The effects from an alteration in the climate system are known as teleconnections. These teleconnections are predicted to shift over particular regions with strong observed precipitation teleconnections (IPCC, 2013).

While there is evidence of anthropogenic forcing affecting much of modern climate change, (IPCC, 2007; Foster and Rahmstorf, 2011) natural variability influences accelerated warming and cooling periods observed in the GMST (IPCC 2007, 2013; Easterling and Wehner, 2009; Meehl et al., 2011; Kosaka and Xie 2013; England et al., 2014; Watanabe et al., 2014). Natural variability can manifest as internal mechanisms operating within the global oceans and atmosphere. These internal mechanisms can vary on seasonal to multi-decadal timescales.

\subsection{Natural Variability}

Typically natural climatological variations are classified by their observed timescales but can also be classified as different spatial patterns (Ghil, 2002). There has been much literature linking natural oceanic variability to changes in air temperatures at the surface (Meehl et al, 2011; Trenberth and Fasullo, 2013; Kosaka and Xie, 2013), 
persistent drought in North America (Enfield et al., 2001; McCabe et al., 2004; Burgman et al, 2010; Burgman and Jang, 2015), Atlantic hurricane intensity and frequency (Knight et al., 2006, Grossman and Klotzbach, 2009) and changes to large-scale atmospheric circulations and low-level clouds over the Eastern Pacific Ocean (Burgman et al., 2008; Clement et al., 2009).

For this study we are interested in looking at natural oceanic variability operating on interannual to multi-decadal timescales. Climatological modes of oceanic variability can be found on decadal and interannual timescales in the Atlantic, Pacific, and Indian Oceans. On decadal timescales, modes include Atlantic Multidecadal Variability (AMV), Pacific Decadal Variability (PDV) and the Indian Ocean Dipole (IOD) (Wu et al., 2003; Xie and Carton, 2004; Deser et al., 2010; Han et al., 2014; Steinman et al., 2015) while the El Niño Southern Oscillation (ENSO) and Tropical Atlantic Variability operate on an interannual timescale (Enfield and Nunez, 2000; Marshall et al., 2001; Sheinbaum 2003).

\subsubsection{Pacific Variability}

Over the Pacific Ocean, the Pacific Decadal Oscillation (PDO) is identified as a distinct triangular shaped SST anomaly pattern along the equator stretching into the central Pacific and a curved area of SST anomalies extending into the tropics and midlatitudes (Mantua and Hare, 2002; Sarachik and Vimont, 2003; Wu et al., 2003; Minobe et al., 2004). An elliptical region of opposite signed SST anomalies in the northern central Pacific surrounds the abovementioned-curved area along the western coast of the United States. An index has been developed by Mantua et al., (1997) calculating the leading Empirical Orthogonal Function (EOF) of monthly SST anomalies in the Pacific 
above 20 degrees north latitude. Despite the index being calculated in the north Pacific, there is an indication similar amplitudes of climate variability also exist in the southern hemisphere (Garreaud and Battisti, 1999). The oscillation period for the PDO has been observed to be between 20-30 years. There is much debate on whether the mechanisms forcing this mode are produced by purely tropical processes (Kirtman 1997; Knutson and Manabe, 1998; Jin, 2001; Wu et al., 2003; Burgman et al., 2008b) or by tropicalextratropical interactions (Gu and Philander, 1997; Kirtman and Schopf, 1998; Kleeman et al., 1999; Schneider et al., 2002; Burgman et al., 2008a; Dommenget and Latif, 2008; Clement et al., 2009) through either oceanic wave and circulation dynamics or atmosphere and ocean teleconnections.

On interannual timescales, ENSO is defined as a strongly coupled mode identified by SST anomalies in the tropical Pacific Ocean and fluctuations in tropical sea level pressure (SLP) between the western and eastern Pacific (Sarachik and Vimont, 2003; Wang et al., 2012). Normal conditions found in the tropical Pacific include cool equatorial waters in the central and eastern Pacific and a warm pool of equatorial waters in the western Pacific (Sheinbaum, 2003). Observed ENSO anomalies oscillate between warm and cool SSTs in this region (Sheinbaum, 2003; Sarachik and Vimont, 2003; Wang et al., 2012). The oscillation period is interannual between 2-7 years. An important aspect of ENSO to remember is, the coupled ocean-atmosphere oscillation includes the El Niño component corresponding to the ocean and the Southern Oscillation representing the atmosphere (Wang et al., 2012). The Southern Oscillation is characterized by an interannual seesaw in tropical SLP between the western and eastern Pacific, consisting of a weakening and strengthening of the easterly trade winds over the tropical Pacific (Wang 
et al., 2012). The fluctuations in SLP are a feedback response to the variations in SST. Ultimately, the changes in SLP and SST are different features (i.e., atmospheric and oceanic respectively) of the same event.

Bjerknes (1969) explains this coupling as a positive feedback between the ocean and atmosphere with easterly winds as a result of zonal SST and pressure gradients across the basin. Dynamical processes in the ocean establish an equatorial cold tongue in the eastern Pacific driven by an eastward sloping thermocline below that provides deep cold ocean temperatures at the surface though upwelling. During warm ENSO events, the easterly winds slacken by a reduction in zonal SST gradients and the thermocline slope relaxes over the eastern Pacific as a result of warmer SSTs. For cool ENSO events, easterly winds are anomalously strong resulting in a steeper sloping thermocline that produces stronger upwelling and cooler SSTs in the eastern Pacific. While the characteristics of ENSO can be easily modeled (Zebiak and Cane, 1987), it is unclear if ENSO is a stochastically driven system or is self-sustained (Bjerknes, 1969; Neelin et al., 1998; Yeh and Kirtman, 2004; Wang, et al., 2012). Understanding how ENSO is driven is useful in identifying the relationship of forcings between modes of variability in the Pacific and their predictability (Kirtman and Schopf, 1998).

\subsubsection{Atlantic Variability}

Variability over the tropical Atlantic is known to influence regional climates over the African and South American continents (Moura and Shukla, 1981; Folland et al., 1986; Nobre and Shukla, 1996; Giannini et al., 2004). The tropical Atlantic region is remotely forced either by the tropical Pacific (Latif and Barnett, 1994; Enfield and 
Mayer, 1997; Klein et al., 1999) combined with local air-sea interactions (Chiang et al., 2002; Huang et al., 2002) or forcing through the extra-tropics and strength of the trade winds (Nobre and Shukla, 1996). An inter-hemispheric mode of SST variability resides over the northern tropical and southern subtropical Atlantic resulting in a dipole-like structure or meridional mode (Moura and Shukla, 1981; Nobre and Shukla, 1996; Chang et al., 1997). There is however no evidence of a significantly coupled dipole mode on interannual or decadal timescales (Enfield and Mayer, 1997; Dommenget and Latif, 2000). Instead, the mode is simply a cross-equatorial SST gradient coinciding with the seasonal meridional displacement of the intertropical convergence zone (ITCZ) (Nobre and Shukla, 1996; Ruiz-Barradas et al., 2000). The dipole mode is a result of low frequency changes in evaporation and is decoupled from variations in the Atlantic thermocline (Carton et al., 1996).

Along the eastern equatorial Atlantic a coupled mode of variability comparable to the Pacific ENSO emerges. The Atlantic coupled mode is often referred to as the Atlantic Niño. The physical mechanisms responsible for the ENSO-like mode in the Atlantic are similar to the dynamical processes found to occur in the equatorial Pacific (Zebiak, 1993; Carton and Huang, 1994; Carton et al., 1996). However dissimilarity in basin size results in shorter duration of warm and cool events and a less robust signal compared to the Pacific. The Atlantic Niño mode is not self-sustaining but shows interannual oscillation favoring a two to four year period (Zebiak, 1993). Despite the vast difference in basin size, displacement of land masses, and lack of a western warm pool linked to convection in the Atlantic, regression analysis shows three components of the Bjerkenes feedback found in the Pacific exist in the Atlantic (Keenyside and Latif, 2007). The 
positive feedback results primarily in peak SST variability in boreal summer. Another peak in variation has been found through satellite and in situ observations to occur in November-December (Okumura and Xie, 2006). A relationship seems to exist between both tropical modes on short interannual and decadal timescales and is associated with shifts in ITCZ locations (Servain et al., 1999)

In the North Atlantic, oscillating warm and cold SSTs fluctuate on average of 4060 years (Delworth et al., 1993; Kushnir, 1994; Schlesinger and Ramankutty, 1994; Goldenburg, 2001; Alexander et al., 2014). The low frequency SST pattern may be a response to high frequency forcings in the atmosphere (Marshall et al., 2001; Clement et al., 2015). However, a close relationship is suggested to exist between changes in Atlantic hurricane intensity and frequency, changes in the hyrdoclimate over the Brazilian rainfall and Sahel desert (Knight et al., 2006), the phase change of North Atlantic SST variability and changes in the intensity of the thermohaline circulation (Delworth et al., 1993; Knight et al., 2005, Grossman and Klotzbach, 2009). Intensity changes of the meridional overturning circulation are driven by density anomalies found in the sinking region located in the North Atlantic Ocean (Delworth et al., 1993). An overall weakening in the overturning circulation produces substantial cooling over the North Atlantic while warming SSTs are associated with a strengthening in the circulation (Delworth and Man, 2000; Zhang and Delworth, 2005). As SSTs warm by anthropogenic forcing, an influx of melted fresh water may weaken the circulation and induce cooling temperatures over the Atlantic. 


\subsubsection{Indian Ocean Variability}

Similar to the Pacific and Atlantic Oceans, the Indian Ocean experiences several modes of variability across the basin. Interannual modes include a basin-wide mode with a warming or cooling pattern associated with changes in cloud cover induced by ENSO (Klein et al., 1999) and sustained by ocean dynamics and local air-sea interactions beyond termination of ENSO events ( $D u$ et al., 2009). A subtropical dipole mode (Behera and Yamagata, 2001) generated by air-sea interactions along the African subcontinent, through atmospheric teleconnections outside the Indian Ocean, and by the Antarctic circumpolar wave (White and Peterson, 1996; Morioki et al., 2012; Morioki et al., 2013) results in positive phases represented by warm SST anomalies in the in the southwestern Indian Ocean and cold anomalies in the eastern Indian ocean (Han et al, 2014). Another dipole structure operates on both an interannual and decadal timescales and is known as the Indian Ocean Dipole (IOD). Varying on interannual timescales, the IOD is self-sustained through strongly coupled ocean-atmosphere dynamics (Webster et al., 1999) resulting in Bjerknes-type feedbacks between equatorial winds and zonal SST gradients (Han et al, 2014). A positive phase of the IOD is denoted by cold SST anomalies in the eastern tropical Indian Ocean and warm SST anomalies along the western tropical edge of the basin (Saji et al., 1999).

Overall, little is known about variability over the Indian Ocean compared to the Atlantic and Pacific basins. Nevertheless, a study done by Krishnamurthy and Krishnamurthy (2016) suggests decadal and interannual components of the IOD to be influenced by wind patterns over the Pacific linked to the PDO and ENSO. The IOD has also been linked to rainfall along the region, monsoon rainfall variability over the Indian 
and Asian peninsula on interannual timescales, and interdecadal modulations of the monsoon related to ENSO (Behera et al. 1999; Ashok et al., 2001; Ashok et al., 2003; Ashok et al., 2004; Yamagata et al., 2004).

\subsection{Background}

The Earth's energy budget is a delicate balance between the incoming radiation from the sun and outgoing longwave radiation from the surface of the Earth, along with convective energy fluxes derived by the surface (Wild et al., 2013; IPCC, 2013). Currently, there is a positive global energy imbalance at the top of the atmosphere of about $0.5-1 \mathrm{~W} / \mathrm{m}^{2}$ (Watts per meter squared) (Trenberth, 2009; Trenberth and Fasullo, 2013; IPCC, 2013). The imbalance should bring about continual warming as seen in past decades as an excess of energy adds heat that becomes absorbed by the land or ocean surface, causing either warming to the surface or melting of snow and ice. With the lack of observed surface heating at the turn of the $21^{\text {st }}$ century denoted by the GMST record (Figure 1.1), Trenberth (2009) suggests a simple rearrangement of energy within the climate system is taking place and the added energy could be traced by changes in the atmosphere, ocean, cloud cover, ice sheets, and aerosols. Two schools of thought attempt to explicate the cause of observed pauses in accelerated warming in the GMST despite a positive imbalance: i) changes in radiative forcing due to changes in stratospheric water vapor (Solomon et al., 2010) and aerosols (Solomon et al., 2011; Kauffman et al., 2011) and ii) changes in oceanic variability (Meehl et al., 2011; Kosaka and Xie 2013).

Radiative forcing of stratospheric water vapor dominates the longwave forcing which can influence temperature changes in the stratosphere and troposphere. Solomon et 
al., (2010) suggest a quiescent period of warming may be a result in a decrease of stratospheric water vapor concentrations after the year 2000. The decrease in water vapor in the stratosphere has been positively correlated with increases in SST in the western warm pool located in the tropical Pacific (Rosenlof and Reid, 2008). The increase in SST in turn affects water vapor entry values and cold point temperatures closely related to rising air. The rising air in active convection transports the water vapor from the troposphere into the stratosphere. Decreases in stratospheric water vapor act to warm the stratosphere but cool the troposphere (Solomon et al., 2010). Despite an expected increase of GHGs, after the year 2000, observed rates of warming in the troposphere might have decreased due to a reduction in stratospheric water vapor.

Another important feature in the stratosphere effecting radiative forcing is the background stratospheric aerosol layer, thought to consist of carbonyl sulfide from volcanic eruptions or anthropogenic sulfur largely because of increases in coal consumption (Solomon et al., 2011). The increase in sulfur emissions also slows the increase in radiative forcing associated with rising GHG concentrations (Kauffman et al., 2011). While the radiative forcing of stratospheric water vapor and sulfur may offset warming, the latter constituent is short-lived in the atmosphere and the two do not account fully for the observed pause in warming. Another factor must be stronger or working in tandem with the effects of radiative forcing for models to fully capture the slowdown in warming.

Alternatively, modes of natural oceanic variability have also been found to influence the observed changes in air temperatures at the surface. Observations and climate models have discovered a sequestration of the excess heat is located in the deep 
ocean below 700 meters (Meehl et al., 2011; Balmaseda et al., 2013; Guemas et al., 2013; Trenberth and Fasullo, 2013; Chen and Tung, 2014; Drijfhout et al., 2014). Overall, deep global oceanic temperatures are warming despite notably cooler temperature anomalies recently observed at the surface. Despite global oceanic warming, each basin experiences different processes leading to warming in the deep ocean. For the North Atlantic in the winter, deep convection weakens, resulting in less cold surface waters subducted which indirectly induces a warming effect at the subsurface and in the deep Atlantic ocean (Meehl et al., 2011).

For the Pacific Ocean, the deposition of heat into the deep ocean may be a result of cold phased anomalies of the PDO where cold SSTs directly cool the air above by removing heat from the atmosphere. The heat is then subducted below into the deep ocean. The cooling temperatures of the tropical Pacific may be associated with prepondering cold ENSO events which has also been observed during the first decade of the $21^{\text {st }}$ century (Meehl et al., 2011; Kosaka and Xie 2013). Also, a substantial acceleration of the Pacific trade winds induces increased equatorial upwelling of cooler SSTs to the surface in the central and eastern Pacific may be the culprit for a slowdown in warming (England et al., 2014).

While natural variability seems to play a role in a slow down in warming, it may also be connected to periods of accelerated warming observed in the temperature record. To further suggest natural variability is a significant contributor to climate change during periods of accelerated warming, the State of the climate report by the National Oceanic and Atmospheric Association (NOAA) has declared 2015 to be one of the warmest years on record. The warming air temperatures may be a result of one of the strongest El Niño 
events recorded. Also, the waters of the North Pacific along the west coast of the United States, where the PDO is calculated, experienced an anomalous warm pool of SSTs that may have been associated with a shift to warmer PDO anomalies during 2015. With the possibility of shifting into a warm phase of the oscillation, there is indication the climate may be entering into another period of accelerated warming.

With anthropogenic forcing projected to increase throughout the next century, it has been indeterminate as to when a full anthropogenic signal will emerge from under the signal of internal forcings. Despite our physical understanding, the largest amount of anthropogenic warming is expected in the polar latitudes. Several studies have shown the earliest emergence of a warming signal is currently occurring first during summer months in lower latitude countries (Mahlstein et al., 2011; Hawkins and Sutton, 2012). The warming in lower latitudes is because the mid-latitudes receive high amounts of atmospheric and temperature variability, in turn causing a delay in the emergence of a warming signal outside of the tropics (Mahlstein et al., 2011). What will happen to the frequency and amplitude of oceanic variability as GHG concentrations increase? There is much discussion as to how the structure of ascribed modes of oceanic variability will respond to warmer GMSTs.

Overall not much research has been done for multidecadal modes of variability. However, the Intergovernmental Panel on Climate Change's (IPCC) fourth assessment report (2013) suggests changes to AMV behavior are unlikely to occur as the mean state of the climate changes by anthropogenic forcing while fluctuations of AMV are likely to continue strongly influencing regional climates. For the Pacific, Furtado et al., (2011) found decadal variability may not exhibit any spatial or temporal changes under larger 
amounts of anthropogenic warming. The lack of changes to decadal variability could be the result of questionable disassociations by how individual models capture the dynamics associated between oceanic modes of variability.

For interannual variability, the IOD is very likely to remain active with unchanged SST variability relative to any given mean state (Cai et al., 2009) despite a reduction in thermocline depth due to changes in zonal gradients (Zheng et al., 2010; IPCC, 2013). The change in thermocline feedback will possibly decrease ENSOs modulation on the IOD (Saji et al., 2006; Zheng et al., 2010). In the Atlantic, a persistent warming trend is already being observed with warming weaker in the North than in the South Atlantic along with a weakening the equatorial cold tongue in the Atlantic Niño region (Tokinaga and Xie, 2011) causing changes in precipitation and atmospheric circulation patterns associated with shifts in the ITCZ (IPCC, 2013). In the tropical Pacific there are many studies suggesting how variability will change on a warmer globe.

Theoretically and through analyses, as the surface warms, the equatorial zonal SST gradient weakens along with a slackening of surface easterly winds resulting in an overall reduction in intensity of the zonal overturning air across the Pacific known as the Walker Circulation (Vecchi et al., 2006, Zhang and Song, 2006). The "Weaker Walker" mechanism is associated with a warm El Niño-like oceanic structure and can be manifested by changes in the global hydroclimate (Knutson and Manabe, 1995; Held and Soden, 2006) or by atmospheric feedback mechanisms (Betts and Ridgeway, 1989; Ramanthan and Collins, 1991; Meehl and Washington, 1996; DiNezio et al., 2009). One particular feedback mechanism hypothesizes an "Atmospheric thermostat" response (Ramanathan and Collins, 1991). Additional warming in the western Pacific results in an 
increase of high cirrus clouds, shielding the surface from insolation resulting in less warming. In the eastern Pacific the cloud shielding effect would be less efficient because of cooler temperatures though upwelling and would instead warm; creating a decrease in easterly winds and an El Niño-like response. Even under quadrupled carbon dioxide simulations the spatial pattern for the tropical Pacific may look like a warm phase of ENSO yet changes in amplitude and frequency depend on how a particular model represents ENSO forcings (Merryfield, 2006; Yeh and Kirtman, 2007).

In contrast, using an ocean model with simplified atmospheric processes, one argument suggests ocean thermodynamic processes produce an "ocean thermostat" response where the oceanic structure takes on a cooler phase of ENSO to moderate increased anthropogenic warming (Clement et al., 1996; Cane et al., 1997). By the ocean thermostat mechanism, as heating increases in the tropics the zonal SST gradient between the eastern and western Pacific strengthens and increases surface easterly winds. The intensification of the atmospheric circulation induces shoaling of the eastern Pacific thermocline generating cooler upwelling from the ocean mixed layer opposing the heating at the surface.

A study attempting to reconcile the two paradigms using both mechanisms, the ocean thermostat and weaker walker circulation, found an El Niño-like pattern may emerge yet not as robust as a consequence of the cooling effects derived by the thermodynamic mechanism originating from the ocean (Vecchi et al., 2008). Considering the mean state becomes more El Niño-like, interannual variability may result in statistically stronger cold events associated with ocean dynamics (Timmerman et al., 1999). However, using an ensemble of complex coupled models, the most likely scenario 
would result in no large amplitude changes towards either warm or cold mean ENSO conditions (Collins, 2005). The lack in ENSO amplitude change occurs because models predict both an amplification of the thermocline response as a result of ocean dynamics along with a weakening of the walker circulation together with responses in the hydroclimate to external forcing (DiNezio et al., 2010).

Therefore, to understand how natural variability will respond to warming global temperatures it is best to look at how observed changes have occurred to these modes since the onset of monotonic warming. One challenge facing researchers is the paucity of observational data in understanding decadal phenomena. Our current instrumental record dates back roughly over a century, documenting two full oscillations between warm and cool phases of AMV and three complete oscillations of PDV. Therefore it is beneficial to examine paleo-proxy based observations from tree rings, ice core data, corals, and various other sources to help identify and analyze multidecadal signals. For example, studies using multi-proxy data and specifically paleo-proxy data from tree rings indicate decadal scale reversals of variability over the Pacific (D'Arrigo et al., 1999; Biondi et al., 2001), Atlantic (Delworth and Mann, 2000; Kilbourne et al., 2008) and Indian Oceans (Zinke et al., 2004; Abram et al., 2007) have occurred at least throughout the last three to four centuries.

Another way to supplement the sparse observed instrumental record is through climate models. Climate models are a valuable resource to researchers with longer integrations of 1000 years or more and at higher spatial resolutions than some observations afford. Data added to climate models are coupled by known complex, physical and dynamical observed processes along with anthropogenic forcings. Models 
vary significantly from observations in representing precipitation and drought patterns, thermohaline circulation strength, and contain biases in SST (Wang et al., 2014), and represent coupled atmospheric-ocean phenomenon differently than observations or even other models (Barnett et al., 1996). The variation between the modeled and observed climate is a result of a combination of errors in simulating external forcing, model response, and internal climate variability (Fyfe et al., 2013). Given these differences, the models do represent internal variability and thus offer valuable insight into the potential changes in natural variability in the future. Moreover, modes of natural variability are spatially related in some ways (Mantua et al., 1997; Mantua and Hare, 2002; Sarachik and Vimont, 2003; Minobe et al., 2004) through direct interactions and atmospheric forcings (Klein et al., 1999; Enfield and Nunez, 2000; Zhang and Delworth, 2007; Hetzinger et al., 2011) helping influence rainfall and drought (Mcabe et al., 2004; Zhang and Delworth, 2006) in the northern hemisphere. Despite possible linkages, climate models seem to keep these phenomena independent (Park and Latif, 2010) which helps to fully understand the mechanisms taking place under the effects of natural variability.

\subsection{Hypothesis}

A leading question is how climate models predict modern anthropogenic climate change but failed to simulate the most recent observed pause in warming. Is it possible to have periods of cooling superimposed on a long-term warming trend? Preliminary analysis of temperature trends in the observational data, CMIP3, and CMIP5 simulations, point to natural variability as a possible source for inconsistencies between the simulated and observed trends of the early $21^{\text {st }}$ century (IPCC, 2013). In spite of many 
improvements to climate models, there remains a large amount of uncertainty in how well models will forecast future climate change. The uncertainty in forecasting the future climate is in part a result of the several different possible emission projections provided by the IPCC along with each climate models response to natural variability on global and regional scales (Deser et al., 2012).

Further examination of model simulations from phase three of Coupled Model Intercomparison Project (CMIP3) produce statistically significant periods of "cooling" or no trend within long term warming (Easterling and Wehner, 2009). The fifth phase of CMIP (CMIP5) provides a framework for coordinated climate change experimentation and promises to yield new insights about the climate system and the processes responsible for the observed climate variability (Taylor et al., 2012). Several recent studies attempt to quantify the probably of warming hiatus' under CMIP5 and found 10 year quiescent periods are likely under present day and accelerated warming conditions (Roberts et al., 2015; Schurer et al., 2015). While, the detection of shorter periods of quiescent warming is expected when compared to the IPCC defined 15-year period, other studies suggest there is no statistical evidence for these "hiatus-like" trends to be unusual in the observed (Rahmstorf et al., 2017) or in a warmer future modeled climate (Li and Baker, 2016). The focus of this dissertation is to understand how periods of quiescent warming change between modeled scenarios.

The hypothesis put forward here is the most recent observed plateau in warming found in GMSTs is not an unusual occurrence and is driven predominantly by natural variability in the global oceans operating together with anthropogenic forcing and is not captured fully in many of the current coupled models. How we will experience future 
periods of stagnant warming (or even a period of apparent accelerated warming) on a warmer globe in the future remains to be seen. Our hypothesis is a threshold in the climate system must exist at some warmer temperature in the future where the signal of natural variability in the GMST will no longer be superimposed on a long-term warming trend thus decreasing natural variability and its impact throughout climate system. Understanding the physical processes driving these phenomena will lead to better understanding and allow for development and betterment of future coupled model simulations. The ultimate goal of this research is to use statistical analysis and advanced modeling experiments to assess the climate under different greenhouse forcing scenarios and evaluate the changes in natural variability as surface temperatures warm.

Objectives of this dissertation:

1) Quantify the probability of observed periods of quiescent warming superimposed in long-term warming in the GMST using simple statistical techniques

2) Examine coupled model output from the CMIP5 archive to identify the response of natural variability to the overall changes under different simulated warming scenarios

3) Statistically assess significance in changes to variability in CMIP5 scenarios

To address the first objective of this analysis a simple statistical analysis is performed. The second and third components of the analysis are more phenomenological in order to identify the physical processes responsible for shifts in the GMSTs in the CMIP5 simulations. We see aggressive warming projections lead to decreases in natural variability. This is a key question to answer in order to help improve model response and 
sensitivity to simulated internal climate fluctuations. In addition to examining the spatial structure of the phenomena (e.g., surface winds and ocean heat content) using correlation analysis and identifying consistencies with the observation data, I will determine how the physical processes are affected by increasing radiative forcing.

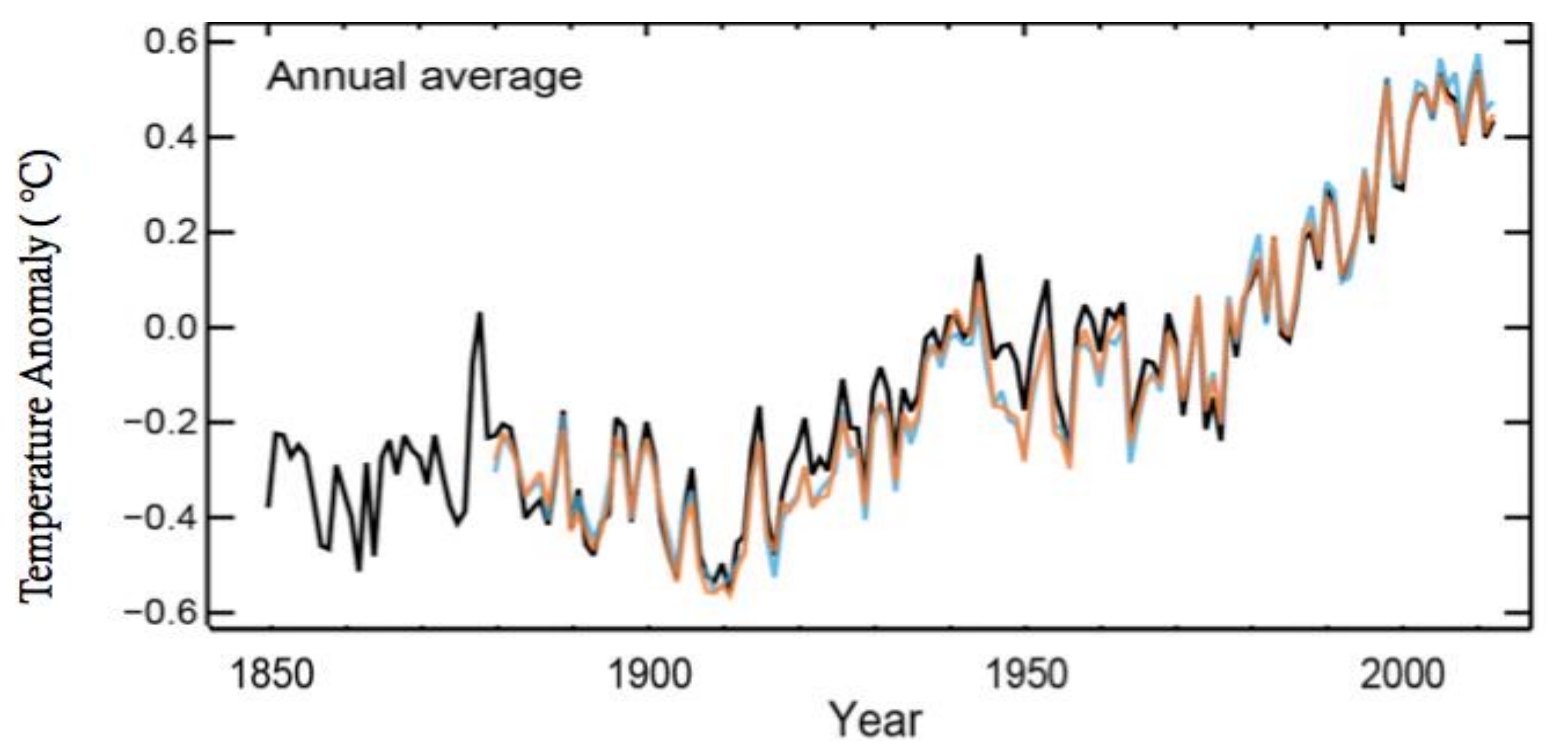

Figure 1.1: Taken from the IPCC report (2013), this figure is the observed global annually averaged combined land and ocean surface temperature anomaly $\left({ }^{\circ} \mathrm{C}\right)$ between 1850-2012. 


\section{CHAPTER 2: OBSERVATIONAL AND MODEL DATA}

\subsection{Data}

\subsubsection{Observational data}

To examine global temperature anomalies, observational datasets from the Hadley Centre's Climate Research Unit HadCRUT4 and ERSSTv4 from Smith and Reynolds (Smith et al., 2008; Morice et al., 2012; Huang et al., 2015) were used for this study. The HadCRUT4 dataset is considered to have restricted spatial coverage, as the data do not employ any form of spatial infilling on gaps, allowing for grid box anomalies to be traced back to the observational records (Morice et al., 2012). The dataset uses a 5-degree horizontal grid of the global historical temperature anomalies with a monthly climatology calculated with respect to the 1961-1990 reference period. The HadCRUT4 dataset is comprised of a blend of land-surface temperature data from CRUTEM4 and SST data from the HadSST3 dataset.

The ERSST datasets are comprised of global monthly sea surface temperatures on a 2-degree horizontal grid with data obtained by the International Comprehensive OceanAtmosphere (ICOADS); a dataset of marine surface data using statistical methods to fill any gaps. Analysis begins from January 1854 to present with monthly anomalies computed with respect to a 1971-2000 monthly climatology.

\subsubsection{Model data}

To examine natural variability, we analyzed SSTs using climate simulations included in CMIP5 which employs several century timescale experiments providing a 
valuable source of climate data to supplement the relatively sparse observational record (Taylor et al., 2012). Analysis of temperature trends in the observational data and CMIP5 simulations, point to natural variability as a possible source for inconsistencies between the simulated and observed trends of the early $21^{\text {st }}$ century (IPCC, 2013). For our analysis, we use annual SST data from a total of 19 ensemble members from the CMIP5 archive (Table 2.1). We used only the first ensemble member (r1i1p1) for each model. For the statistical analysis, a subset of 18 of the models was also used to also evaluate annual air temperature at the surface (TAS) alongside annual SST data.

We evaluated six different types of simulation experiments found within the CMIP5 multi-model archive. Experiments include: (1) a multi-century control run that holds radiative forcing constant at pre-industrial levels of 280 parts per million (ppm) throughout the entire simulation. These simulations are useful because they provide information on internal noise in the climate system. (2) Historical runs that cover the last 150 years from 1850 to 2005 and forced with observed changes in atmospheric composition; forcing includes both natural internal variability, solar, and volcanic forcings along with additional anthropogenic sources. (3) Four different century scale future projections out to 2100 that are numbered according to the estimated peak of radiative forcing in Watts per meter squared $\left(\mathrm{Wm}^{-2}\right)$ throughout the century relative to the pre-industrial value. These simulations are known as Representative Concentration Pathways (RCP) (Moss et al., 2010; van Vuuren et al., 2011).

The RCP simulations give several possible scenarios of GMST change formulated by several assumed prospective mitigation strategies (Figure 2.1). They emphasize forcing projections realized under more than one underlying socioeconomic scenarios. 
Essentially they are representative of a combination of technological development, policy, energy, and land-use changes in the literature needed to achieve and maintain the amount of radiative forcing levels targeted (Masui et al., 2011; Riahi et al., 2011; Thompson et al., 2011; Van Vuuren et al., 2011). We looked at all four different RCP experiments: RCP2.6, RCP4.5, RCP6.0, and RCP8.5 as they vary between the most conservative and liberal amounts of projected radiative forcing to occur during the $22^{\text {nd }}$ century. For example, the pathway for RCP2.6 is to peak with a maximum radiative forcing of $3.0 \mathrm{Wm}^{-2}$ close to mid-century and decline to $2.6 \mathrm{Wm}^{-2}\left(\sim 490 \mathrm{ppm}\right.$ of $\mathrm{CO}_{2}$ equivalent) by 2100 . RCP4.5 ( $\sim 650 \mathrm{ppm}$ of $\mathrm{CO}_{2}$ equivalent) and RCP6.0 ( $\sim 50 \mathrm{ppm}$ of $\mathrm{CO}_{2}$ equivalent) representing medium-low and medium-high projections, respectively, follow a pathway that stabilize without overshooting after reaching their corresponding projected radiative forcing, while the RCP8.5 represents the highest forcing projected to reach a level of radiative forcing of $8.5 \mathrm{Wm}^{-2}\left(\sim 1370 \mathrm{ppm}\right.$ of $\mathrm{CO}_{2}$ equivalent) at 2100 by rising steadily throughout the next century. Temperature anomalies for each simulation in Figure 2.1 follow the projected radiative forcing throughout their respective simulations. 


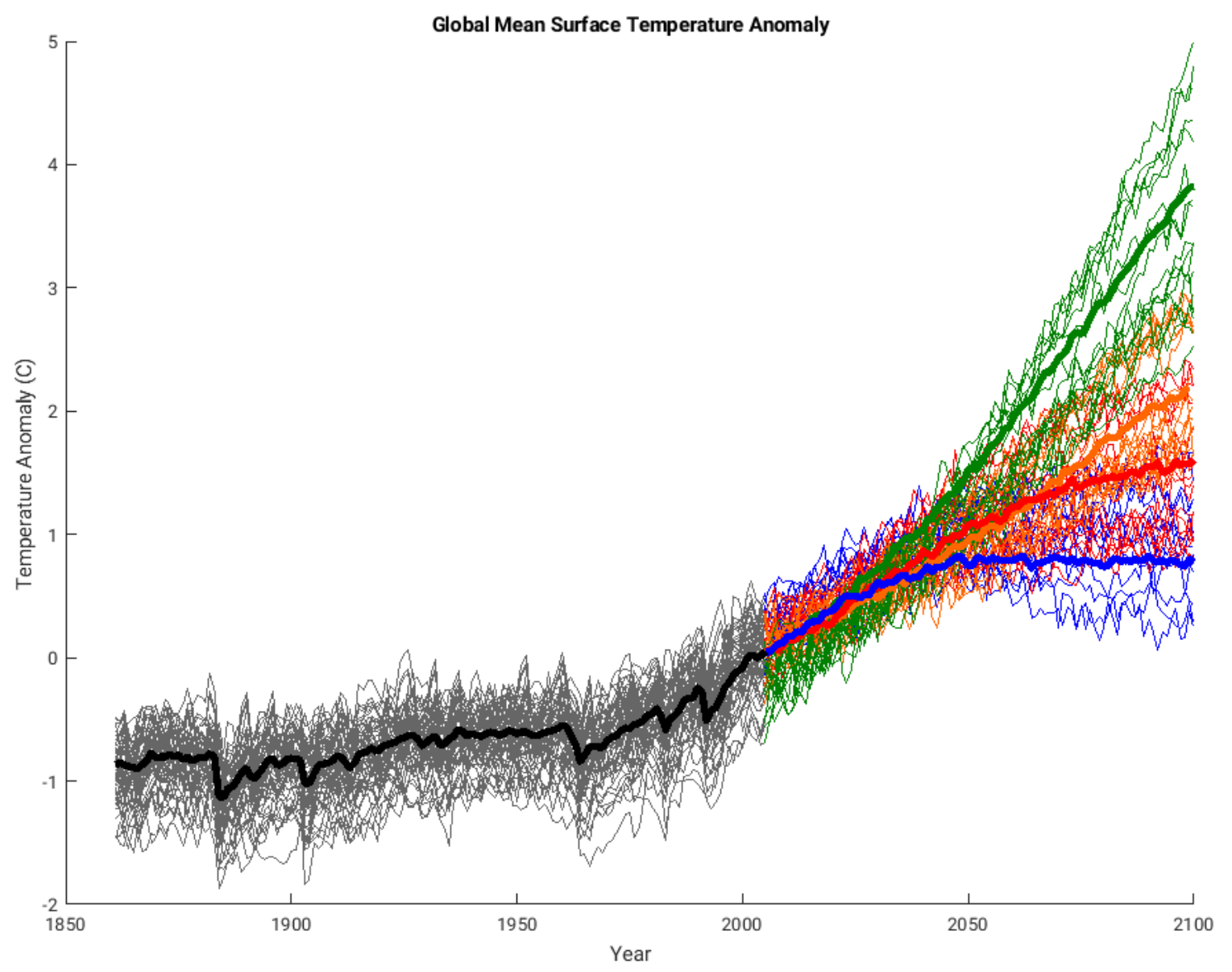

Figure 2.1: Time series of global mean surface temperature anomalies for 18 CMIP5 models. Shown are the time series' for the individual models under the Historical experiment (grey) between 1861-2005 and the four future warming experiments RCP2.6 (dark blue), RCP4.5 (red), RCP6.0 (orange), RCP8.5 (dark green) from 2006-2100. Also depicted are the multi-model averages for each experiment (thick lines). 


\begin{tabular}{|l|l|}
\hline \multicolumn{1}{|c|}{ Model Name } & \multicolumn{1}{c|}{ Modeling Institute } \\
\hline $\begin{array}{l}\text { BCC-CSM1.1-m } \\
\text { BCC-CSM1.1 }\end{array}$ & Beijing Climate Center, China Meteorological Administration, China \\
\hline CCSM4 & NCAR, USA \\
\hline CESM1-CAM5 & $\begin{array}{l}\text { NSF, Department of Energy, and National Center for Atmospheric } \\
\text { Research, USA }\end{array}$ \\
\hline FIO-ESM & The First Intitude of Oceanography, Japan \\
\hline $\begin{array}{l}\text { GFDL-CM3 } \\
\text { GFDL-ESM2G } \\
\text { GFDL-ESM2M }\end{array}$ & NOAA Geophysical Fluid Dynamics Laboratory, USA \\
\hline $\begin{array}{l}\text { GISS-E2-H } \\
\text { GISS-E2-R }\end{array}$ & NASA Goddard Intstitute for Space Studies, USA \\
\hline HadGEM2-AO & $\begin{array}{l}\text { National Institute of Meteorological Resears/ Korea Meteorological } \\
\text { Administration, Korea }\end{array}$ \\
\hline HadGEM2-ES & Met Office Hadley Centre, UK \\
\hline IPSL-CM5A-LR & Institut Pierre-Simon Laplace, France \\
\hline $\begin{array}{l}\text { MIROC5 } \\
\text { MIROC-ESM } \\
\text { MIROC-ESM-CHEM }\end{array}$ & $\begin{array}{l}\text { The University of Tokyo, National Institute for Environmental Studies, } \\
\text { and Japan Agency for Marine-Earth Science and Technology, Japan }\end{array}$ \\
\hline MRI-CGCM3 & Meteorlogical Research Institute, Japan \\
\hline $\begin{array}{l}\text { NorESM1-M } \\
\text { NorESM1-ME }\end{array}$ & Norwegian Climate Centre, Norway \\
\hline
\end{tabular}

Table 2.1: 19 coupled climate models from the CMIP5 archive that provided the first ensemble member (r1i1p1) for the PiControl, Historical, RCP2.6, RCP4.5, RCP6.0, and RCP8.5 experiments. 


\section{CHAPTER 3: QUANTIFIYING THE PROBABILTY OF REDUCED PERIODS OF WARMING}

\subsection{Overview}

Here, we evaluate how natural variability in global mean surface temperatures will be affected as the amount of well-mixed greenhouse gases continues to increase in the atmosphere. By analyzing the global mean surface air temperature and sea surface temperatures under several different scenarios, we hope to elucidate the potential changes in global surface temperatures and determine if and when quiescent periods of warming like the aforementioned "warming hiatus" may cease to occur. Our approach includes a statistical analysis of the global mean surface air (measured at 2 meters) and sea surface temperature time series under 6 different radiative forcing experiments. The analysis includes an examination of the likelihood of similar periods of quiescent warming to the warming hiatus and an analysis of changes in variance of the global mean surface temperatures.

\subsection{Methods}

\subsubsection{Monte Carlo Sampling}

To quantify the probability of a period of stagnant warming as observed at the turn of the $21^{\text {st }}$ century, a statistical analysis of annually averaged global mean surface temperature (GMST) was performed on the observational record from which the warming hiatus mentioned in the recent IPCC report was calculated. We then performed 
the same analysis on 18 of the individual CMIP5 models from Table 2.1 for a period of steady (time dependent) radiative forcing at preindustrial levels $(\sim 280 \mathrm{ppm})$, or the preindustrial control (PiControl) experiment in addition to a simulation with observed estimates of the transient radiative forcing and external forcings (e.g. solar variability and volcanic eruptions) over the past 150 years, or the Historical simulations. To understand how future anthropogenic warming may affect natural variability in the GMST time series, we also examined the probability of another warming hiatus in four experimental simulations with differing suppositions about how society will respond to observed increases in greenhouse gases (GHG) and climate change. These include the RCP2.6, RCP4.5, RCP6.0, and RCP8.5 experiments mentioned in Chapter 2. To better capture the natural variability in the presence of persistent increases in GHGs, we also incorporate different methodologies for removing the warming trends observed in the GMST in the Historical and future warming scenarios. A resampling method known as Monte Carlo sampling was then applied to the observational and CMIP5 model output to help determine how natural multi-decadal shifts change as the radiative forcing changes within the simulations. The Monte Carlo sampling technique uses repeated random sampling with replacement from the population or from a synthetically produced time series to compute the statistic of interest. Monte Carlo sampling methods store the statistic of interest from the sampled data and a probability distribution is then calculated. The distribution reveals the likelihood of the chosen statistic, in this case the decadal trend associated with stagnant warming in the GMST, occurring for the period of interest. Following the IPCC's example, we resampled 15-year "chunks" from the GMST time series and calculated the linear trends for 10,000 iterations over the observational and 
modeled datasets before and after detrending. Recall the 15-year sample coincides with the duration of the most recent reduced period of quiescent warming observed from 1998 to 2012, known as the "hiatus". Rahmstorf et al., 2017 used a similar Monte Carlo approach to assess the significance of trend changes in the observational record and found that a low amplitude trend period as observed is to be expected and argued that no significant trend change has been observed since the acceleration in warming in the 1970s. Note, their analysis only simulated the global surface temperature from 1972-2014 and linearly detrended using a baseline period from 1972-2000. Here we sample over the entire observed HadCRUT4 period from 1850 to 2017.

Utilizing the Monte Carlo resampling technique for 15-year trends within the observed HadCRUT4 record yields a large positive 15-year trend [-0.5 to 0.15$]$ compared to the IPCC calculated trend of 0.05 degrees Celsius $\left({ }^{\circ} \mathrm{C}\right)$ per decade. The median 15 -year trend from the sampled distribution for the HadCRUT4 time series is calculated at 0.078 ${ }^{\circ} \mathrm{C}$ per decade (Figure 3.1). The probability distribution function of the Monte Carlo sampling of the observed GMST in Figure 3.1 does not resemble a normal statistical Gaussian bell curve where the calculated sampled trends fall within one standard deviation of the mean. Instead, the probability distribution is negatively skewed at -0.491 with the median of the sampled 15-year trends higher than the observed hiatus trend highlighted by the red line. The skewness here tells us that any deviations from the median are more likely to be negative (Bancos et al., 2011). With only 168 years of observed data, and considering the paucity of data prior to the 1950 s, there are simply not enough data to sample confidently. To supplement the lack of available observed data for 
our analysis we created a synthetic time series modeled to have similar characteristics to the observed record.

There is much debate on the stationarity of the GMST and the type of trend imparted on the time series by radiative forcing. A stationary process is a stochastic process in which its statistical properties such as its mean and variance do not change over time. Kauffman et al., (2010 and 2013) suggest that it is possible to model GMST as a trend stationary process. The use of linear least squares regression in the removal of the observed trend has been used in several previous studies to calculate the global sea level trend over the past century (Gornitz et al., 1982), to estimate the ENSO signal in global surface temperatures (Wigley, 2000; Santer et al., 2001; Trenberth et al., 2002), to isolate long term trend patterns in global sea surface temperature (Barbosa and Anderson, 2009), to test the independence of surface air temperature to sea surface temperature (Comrie and McCabe, 2012), and calculate the AMO time series (Kerr, 2000) as well as its relationship to rainfall and riverflow patterns over North America (Enfield et al 2001). When calculated globally, natural temperature fluctuations superimposed on a linear anthropogenic trend appear to be more significant and easier to detect (Lenhurtz and Bunde, 2009). In spite of this, some studies suggest the removal of a linear trend is inadequate because global temperatures appearing to have a stochastic trend associated with variability around a nonlinear deterministic trend (Estrada and Perron, 2016; Lai and Yoon, 2018). Huang et al., 1998 and Wu et al., 2007 also argue the trend can be represented as an intrinsically determined monotonic curve, having at most one extremum within a given time span. To determine which detrending method is most 
appropriate for our analysis, we plotted the linearly and nonlinearly detrended HadCRUT4 global mean surface temperature anomaly (GMSTA) together with the observed time series (Figure 3.2). Here, we demonstrate and confirm the difficulty in separating natural variability from the deterministic trend imparted on the global surface temperature as both the linearly (blue line) and nonlinearly (orange line) detrended time series' display similar characteristics to one another. From Figure 3.2, it is inconclusive as to what trend removal technique is best for analyzing changes to natural variability. Because of the simplicity and the efficient use of data under linear least square regression, we choose that method for the purposes of our investigation in this chapter.

Some studies have suggested that GMST can be modeled as a first order Autoregressive (AR1) process (Frankze, 2012; Vyushin et al., 2012; Østvand et al., 2014; Rypdal et al., 2015). An AR1 process (Hasselman, 1976) is a well-known random process that simplistically represents the myriad stochastic processes in the climate system, as it expresses a time series as a linear function of its past values. An AR1 process is defined numerically as:

$$
X_{t}=\alpha_{1} X_{t-1}+\varepsilon_{t}
$$

Where $X_{t}$ is the condition at the current state, $X_{t-1}$ is the condition from the previous state, $\varepsilon_{t}$ is some random Gaussian white noise not correlated to the current state, and $\alpha_{1}$, which is a constant autoregressive coefficient. In this case, $\alpha_{1}$ is known as the lagone autocorrelation coefficient. The autocorrelation tells us how well correlated the current value is to the value from the previous time step. For a time series $X_{1}, X_{2} \ldots X_{\mathrm{N}}$ the lag- $k$ autocorrelation can be calculated as 


$$
\alpha_{k}=\frac{\operatorname{Cov}\left(X_{t}, X_{t-k}\right)}{\operatorname{Var}\left(X_{t}\right)}
$$

Where

$$
\operatorname{Cov}\left(X_{t}, X_{t-k}\right)=\frac{1}{N-1} \sum_{t=k+1}^{n}\left(X_{t}-\bar{X}\right)\left(X_{t-1}-\bar{X}\right)
$$

And

$$
\operatorname{Var}\left(X_{t}\right)=\frac{1}{N-1} \sum_{t=1}^{n}\left(X_{t}-\bar{X}\right)^{2}
$$

Note that $\bar{X}$ is the mean of $X_{t}$ in both equations 3.3 and 3.4. Since we are looking at the GMST as an AR1 process we are only concerned with $\alpha_{k}$ calculated at $t=1$. With an AR1 process assuming stationary values throughout the time series, the autocorrelation must be less than 1. For the observed and linearly detrended HadCRUT4 record, we tested the autocorrelation function and plotted a sample autocorrelation plot for the first 100 lags (Figure 3.3). For the original time series in the top panel of Figure 3.3 we see a strong correlation at lag 1 by 0.898 and declining slowly until the correlation becomes negative at $t=67$ and increases as a negative autocorrelation. A decreasing autocorrelation pattern indicates a strong autocorrelation stipulating a process of high predictability if modeled correctly. For the linearly detrended time series in the bottom panel of Figure 3.3, the lag-one autocorrelation is 0.750 and also displays a slowly declining autocorrelation. However the autocorrelation becomes negative earlier at lag $t=27$ and another negative trending lag at $t=68$. The decreasing autocorrelation confirms an AR1 process is a good fit for our analysis on the GMST. To comply with the 
stationarity constraint of the AR1 process we model the linearly detrended HadCRUT4 time series.

The simple AR1 model will help to estimate the evolution of natural variability in the GMST by creating multiple synthetic simulations of the observed record. In turn, elucidating the likelihood of similar multi-decadal shifts for thousands of simulated years. Figure 3.4 is an example of how well the AR1 time series captures the key features of the observed detrended GMST from the HadCRUT dataset. The blue curve is the linearly detrended HadCRUT time series and the orange curve is one replication (chosen by the author) from the AR1 model on the observed record. It is evident that the AR1 modeled GMST is capable of reproducing the characteristics of the detrended observed time series throughout the entire record. For our analysis we have created 10,000 synthetic records and sampled 15-year trends over these periods.

\subsection{Results}

\subsubsection{Temperature at the Surface ( 2 meters)}

We begin comparing the overall distribution of 15 -year trends between the 18 individual model members for temperature at the surface ( 2 meters) to the 15 -year trends found in Monte Carlo sampled observation and AR1 modeled HadCRUT4 record. The comparisons between the distribution of 15-year trends from the observed and synthetically modeled time series are to see if the probability of a warming trend is akin to a trend observed over the hiatus period. To better directly compare the probability distribution functions for the individual models, Box and whisker plots were created. In 
Figure 3.5, the box encompasses the interquartile range (IQR), explaining $50 \%$ of the data while the median 15-year trend for each model is denoted as a red line in the box. The whiskers represent the extreme highest and lowest numbers and anything beyond (1.5 x IQR) the whiskers of the boxplots are considered outliers. Figure 3.5 shows the sampled 15-year trends over the modeled PiControl experiment for 18 CMIP5 models compared with the 15-year trends sampled over the observed HadCRUT4 time series before linearly detrending. For the PiControl simulations, we resampled over the last 100 years in each ensemble member to avoid sampling over any possible model spin up. As previously mentioned, the observed HadCRUT4 15-year trends display a negatively skewed distribution (-0.491) and the median 15-year trend of $0.078{ }^{\circ} \mathrm{C}$ per decade. For the 18 individual CMIP5 ensemble members, the median 15-year trends all fall below the IPCC calculated hiatus trend of $0.05^{\circ} \mathrm{C}$ per decade from 1998-2012 (indicated by the green line). Also note, for all 18 PiControl models, the probability of 15 -year periods with little to no warming is high as $78 \%$ of the ensemble members underestimate natural variability with a smaller IQR compared to the observed 15-year trends. Moreover, only $22 \%$ of the ensemble members display the IPCC calculated hiatus trend within their upper quartile while the remaining ensemble members have the IPCC hiatus trend within their IQR. The capture of the hiatus trend of quiescent warming is not surprising considering for the PiControl simulation the radiative forcing is held steady at preindustrial levels of $280 \mathrm{ppm}$ for the entire simulation. In the absence of transient radiative forcing, any persistent trend in the time series would likely be indicative of a more systematic error in the model leading the coupled model's climate to "drift". 
Figure 3.6 shows the sampled 15 -year trends over the time series of 18 ensemble members' Historical experiment compared with the observed 15-year trends in HadCRUT4 before linearly detrending and synthetically modeling. The green line denotes the IPCC calculated hiatus trend at $0.05^{\circ} \mathrm{C}$ per decade. Here, all ensemble members display a positive median 15-year trend with the exception of NorESM1-M displaying a negative median 15-year trend. While all of the ensemble members display positive median 15-year trends, GISS-E2-H and IPSL-CM5A-LR display larger median 15-year trends than the observed HadCRUT4 at $0.089^{\circ} \mathrm{C}$ and $0.087^{\circ} \mathrm{C}$ per decade, respectively. GISS-E2-H is the only model under the Historical simulation to display the IPCC calculated hiatus trend outside of its IQR. Next, we linearly detrended the data to remove the anthropogenically-forced signal from the time series and re-tested the probability of a 15-year hiatus for the AR1 simulated time series for each Historical experiment and compared them to the AR1 modeled HadCRUT4 15-year trends (Figure 3.7). Note the calculated IPCC hiatus trend of $0.05^{\circ} \mathrm{C}$ was calculated in the presence of transient forcing as a result of the observed increases in well-mixed GHGs during the $20^{\text {th }}$ century. If the observed data were detrended using a linear least squares method, the Hiatus trend would be reduced to $0.002{ }^{\circ} \mathrm{C}$ per decade and is represented by the green line in Figure 3.7. Here, 10 ensemble members display a negative median 15-year trend following the AR1 modeled HadCRUT4 median 15-year trend. The distribution of the AR1 synthetically derived observational time series now has a median 15 year trend of $0.031{ }^{\circ} \mathrm{C}$ per decade while still negatively skewed. Also, all 18 synthetically modeled distributions contain the calculated detrended IPCC trend over the calculated over the hiatus period well within their IQRs. Simulating a hiatus-like trend in the synthetically 
modeled time series further indicates that decadal cooling within the original record is likely and not an unexpected event. Note only $33 \%$ of ensemble members display a median 15-year trend warmer than the value for the observed detrended hiatus period, further suggesting that the warming hiatus was neither, unique or unexpected over the Historical period.

Next, we resampled the GMST for the original and linearly detrended RCP2.6 and RCP8.5 experiments. We chose the RCP2.6 and RCP8.5 scenarios for this study because they represent the most conservative and liberal radiative forcing projections of the four RCP scenarios, respectively. The comparison between the two RCP scenarios was designed to give us an indication of the range of the possible variations in 15-year trends among the RCP scenarios. Figure 3.8 shows the distributions of the 15-year GMST trends sampled from the full record of the simulation over 2006-2100 for the RCP2.6 (red) and RCP8.5 (blue) scenarios before linearly detrending. With the trend associated with the radiative forcing still in the data, RCP8.5 exhibits no hiatus-like trends compared to RCP2.6. For RCP2.6, all model members contain the hiatus trend of $0.05{ }^{\circ} \mathrm{C}$ within either their IQR or within the tail of the distribution. We also note larger tails for the individual models RCP2.6 compared to their RCP8.5 simulation. Overall, examining Figure 3.8 we find it difficult to compare the 15-year trends between these two scenarios. Therefore, Figure 3.9 presents the distributions of the 15-year GMST trends sampled from 20062100 of the linearly detrended record for the RCP2.6 (red) and RCP8.5 (blue) scenarios. As with the detrended observed and Historical experiments, both RCP experiments have median values of 15-year trends near zero and well within the IQRs for all models; 
consistent with the null hypothesis where periods of quiescent warming are an unlikely event in the future given a large increase in the radiative forcing in the RCP8.5 simulations. Compared to the Historical experiment which saw 10 members exhibiting negative median 15-year trends, 39\% of the ensemble members for RCP2.6 display negative values for the median 15 -year trends while $33 \%$ of the ensemble members from RCP8.5 result in a negative median 15-year trend. For the Monte Carlo sampling of the RCP simulations, larger variance in GMST trends within the interquartile range from RCP2.6 to RCP8.5 is seen for $61 \%$ of the model members. They display a decrease in the range of 15-year trends within the IQR to their respective RCP8.5 run. The largest single reduction in variance between the two experiments among member models is found in GFDL-ESM2M by $38.22 \%$. For the $61 \%$ of ensemble members mentioned above, the drop in variance between their RCP2.6 and RCP8.5 IQR are significant at the 95\% level of a two-tailed F-Test. While 13 members display a decrease in variance, the box and whisker plots in Figure 3.9 indicate an increase in variability of GMST trends in 28\% of the ensemble members for RCP8.5 scenarios, with the largest increase in variance noted in BCC-CSM1.1-m (a 37.7\% increase). The increases in variance are also found to be statistically significant from a two-tailed F-test. With $61 \%$ of ensemble members displaying a decrease in the range of 15 -year trends with increased future GHGs may suggest that the signal for internal variability will become overwhelmed over time particularly in the RCP8.5 simulations where the larger amplitude of forcing may begin to dominate. The result of internal variability being overwhelmed in RCP8.5 is consistent with the results of Li and Baker (2016), who performed a statistical meaningful test and found hiatus-like 15-year trends for 38 individual model members are likely to continue 
under RCP4.5 and are less likely to occur in RCP85. We note that Li and Baker did not incorporate the RCP2.6 or RCP6.0 simulations in their study. Previous studies have indicated this may already be taking place in the tropics where the signal for anthropogenic climate change is argued to overwhelm the signal of natural variability decades sooner compared to mid and polar latitudes (Mahlstein et al., 2011; Hawkins and Sutton, 2012). It is important to note that different coupled models simulate natural variability differently (Tebaldi and Knutti., 2007; Hawkins and Sutton., 2009; Deser et al., 2012). Therefore, differences in the mechanisms responsible for natural variability may also be the reason for the large variability between the RCP experiments.

To elucidate the variability among the two scenarios we next subset the data by dividing the simulation period in half and resampled using the same Monte Carlo sampling procedure over both portions of the century within each model. The analysis between the first and latter half of the modeled future allows us to evaluate how the 15year trends change from the first half (2006-2050) to the latter half (2051-2100) of both RCP simulations where there is a notable differences in the amplitude of radiative forcing. Figure 3.10 shows the results of Monte Carlo resampling using the RCP2.6 GMST for both halves of the modeled century. The boxplot results for the first half are denoted in red and those for the latter half in blue. Variability captured by the IQR drops in the latter part of the record for $67 \%$ of the ensemble members. The largest decrease in variance in the latter half of the record is $79.7 \%$ in HadGEM2-ES. When tested for statistical significance the largest decrease in the IQR in the last 50 years is statistically significant at a 95\% significance level from a two-tailed F-test. 
Figure 3.11 shows the same calculation applied to the 15-year trends of GMST under RCP8.5 radiative forcing. Similar results were not found for RCP8.5 as in RCP2.6 with respect to the drop in variance during the latter half of the century. Only $44 \%$ of ensemble members show a decrease in variability in RCP8.5 in the latter portion of the record. However, the IQRs in the RCP8.5 15-year trend calculations are smaller for approximately $72 \%$ of the ensemble members than their IQRs for RCP2.6 in the first half of the record. The largest reduction in variance between the first and second portion of the RCP8.5 century is noted in IPSL-CM5A-LR by 63.7\%. Again, the largest decrease in variance in statistically significant at a $95 \%$ significance level. To identify the possible cause in the large ranges in size of the IQRs among experiments and individual models, we calculated the correlation between the IQR and variance within each model. Figure 3.12 is a scatter plot of the correlations for the PiControl, Historical, RCP2.6 and RCP8.5 experiments. The PiControl and Historical experiments have a weak positive correlation of $r=0.48$ and $r=0.45$ between the amount of variance within the models to the size of the IQR of the 15-year trend, respectively. Both RCP2.6 and RCP8.5 have a moderate positive correlation of $\mathrm{r}=0.73$ and $\mathrm{r}=0.67$, respectively. All correlations but the Historical experiment are statistically significant. While there is a correlation between the amount of variance each model contains to the range in possible 15-year trends, it is also possible the internal mechanisms that may be driving the large increase in 15-year variability within RCP8.5 may be a result of the simplicity of the analysis but is out of the scope of this chapter and will be analyzed more in depth in the Chapter 4 of this dissertation. 
Next, we looked at the 18-member multi-model statistics. Here we have included all four future warming scenarios to compare how variability changes within simulations with steady (time independent, PiControl) forcing to experiments that contain transient forcing with differing amplitudes. The incorporation of the multi-model analysis stems from several studies that suggest that individual model errors are compensated for when assessed over multiple models (Haegorden et al., 2005; Palmer, 2005; Tebaldi and Knutti, 2007; Reichler and Kim., 2008; Weigel., et al, 2008). Multi-model ensembling is different than single-model ensembling. The latter is primarily used to parse out uncertainties and biases within a particular model that arise from the models physics and parameterization schemes. These uncertainties are addressed by running ensemble forecasts from different initial conditions. The multi-model ensemble combines model simulations from structurally different models where one or more initial conditions are available from each model (Tebaldi and Knutti, 2007). Multi-model ensembling has been found to be skillful in sub-seasonal to seasonal (Krishnamurti et al., 1999; Yun et al. 2003; Palmer, 2004; Palmer et al. 2005; Weisheimer et al., 2009) and recently in decadal predictions (van Oldenborg et al., 2012; Smith et al., 2013). To calculate the multi-model statistics we combined the individual detrended annual GMST for each of the six experiments through concatenation and resampled the 15-year trends for 10,000 iterations over the multi-model time series. Combining the 18-member ensemble gives us 1800 simulated years to sample over for the PiControl, 2610 for the Historical, and 1710 for all four RCP experiments. 
Figure 3.13 shows the probability distribution of 15 -year trends over the multimodel concatenated GMST time series for each of the six simulations. Included in the plots is a red line highlighting the calculated trend for the observed Hiatus of $0.05{ }^{\circ} \mathrm{C}$ mentioned in the IPCC report and estimated from 1998 to 2012 in the observed HadCRUT4 time series. For the distributions of the PiControl, Historical, and RCP2.6, the HadCRUT4 calculated trend falls well within one standard deviation of the mean of the distribution, indicating the likelihood of continued periods of reduced warming within these experiments. However, as the radiative forcing increases in RCP4.5, RCP6.0, RCP8.5 scenarios, the distribution begins to shift towards warmer 15-year trends. Thus the calculated 15-year trend for the hiatus period begins to shift toward the tail of these distributions as the radiative forcing increases, further suggesting that quiescent periods like the recent "hiatus" in the GMST may become increasingly unlikely to occur as the concentrations of well-mixed GHGs increase in the future.

Next we wanted to compare the probability of 15 -year trends over only the first 50 years of ensemble members (Figure 3.14 ) to the probability of a 15-year trend in the last 50 years of each ensemble member (Figure 3.15). When sampling over the first half of the observed HadCRUT4 times series (1900-1950), the median 15-year trend is found to be $0.002^{\circ} \mathrm{C}$, analogous to the 15 -year trend calculated over the detrended hiatus period (1998-2012). Before sampling over the multi-model time series, sub-setted GMSTs were concatenated using the first and last 50 years for each ensemble member respectively. In Figure 3.14, over the first half of the record similar results are found when compared to Figure 3.13 with respect to the PiControl and Historical. For the first half of the century RCP2.6, RCP4.5, and RCP6.0 display peak 15-year trends warmer than the observed 
hiatus trend while "hiatus-like" trends become completely diminished as the amplified forcing increases in RCP8.5. Interestingly, when looking only over the first 50 years, the distribution for the Historical experiment displays a normal Gaussian distribution when compared to the full record of the Historical simulation, which exhibits a more leptokurtic distribution. The difference in kurtosis may a result of the possibility of less outlier's in the earlier part of the record, as most of the amplified forcing does not emerge in the Historical time series until the latter half of the century (Figure 2.1). Another interesting note is the large positive skewness for RCP8.5 (0.782) over the first 50 years and RCP2.6 with the second largest positively skewed distribution (0.406).

Figure 3.15 shows the distribution of 15 -year trends over the concatenated GMST of the last 50 years from 18 ensemble members. The 15 -year trends sampled over the latter half of the observed HadCRUT4 times series (1951-2017), are again found to be comparable to the 15-year trend calculated over the detrended hiatus period (1998-2012) along with a strong negatively skewed distribution at -0.796 . Over the latter half of the record, all six CMIP5 experiments display normal Gaussian distributions with a kurtosis of 3. The probability of a trend comparable to the hiatus is still likely over the latter half in the PiControl experiment with a higher probability of negative 15 -year trends in RCP2.6 and a higher probability of positive 15-year trends in the Historical, RCP4.5, RCP6.0 and RCP8.5. A hiatus-like trend is not unexpected in the in the latter half of the PiControl simulation, as the radiative forcing does not change over time. For the Historical period, a 15-year trend equivalent to the recent warming hiatus is also highly probable. Again, as the concentration of GHGs increase in the atmosphere, the analysis 
indicates that a hiatus-like trend becomes increasingly less likely at the end of the $22^{\text {nd }}$ century in RCP6.0 and very unlikely to occur under RCP8.5 concentrations.

Figure 3.16 compares the probability distributions of 15 -year trends for the six CMIP5 experiments after removing a linear trend in the form of a boxplot. For the multimodel analysis, the median 15-year trend for each of the CMIP5 experiments fall closer to zero than many of the individual members as seen in previous figures. Sampling over the entire record results only in RCP2.6 to exhibit negative 15-year trends with a median 15-year trend of $-0.010{ }^{\circ} \mathrm{C}$ per decade. The size of the IQR increases from the PiControl to the Historical scenario by $46.4 \%$ along with an increase in the extremes. The increase found may be a result of the addition of observed changes to solar and volcanic forcing added in the Historical experiment or possibly the modeled internal mechanisms driven by natural variability operating in the oceans. From the Historical to the RCP experiments, the size of the IQR decreases by $10.3 \%$ to $\mathrm{RCP} 2.6,24.0 \%$ to $\mathrm{RCP} 4.5$, $39.1 \%$ to RCP6.0 and $17.2 \%$ to RCP8.5. The increase in IQR between RCP6.0 and RCP8.5 is found to be significant at the $95 \%$ level of a two-tailed F-Test. In fact, all changes in variability between the multi-model CMIP5 experiments are significant with the exception for the increase in variability found in the IQR between RCP4.5 and RCP8.5. Despite the increase in IQR from RCP4.5 and RCP6.0 to RCP8.5, there is still an overall decrease in variability noted within the extremes and IQR from RCP8.5 to both the Historical and RCP2.6 experiments.

The same concatenation method was used for the first half of each individual ensemble GMST before sampling over the multi-model GMST. Figure 3.17a shows the 
15-year trends for the first half of each ensemble member. Again, from the PiControl to the Historical there is a notable increase in both the extremes and IQR. From the Historical to RCP2.6 there is a $4.8 \%$ increase followed by a drop in both the extremes and IQR to RCP4.5 and RCP6.0, followed by an increase again in extremes and IQR for the RCP8.5 simulations. However, for all future scenarios, the median 15-year trends are comparably cooler than the Historical experiment. The multi-model analysis for the last 50 years of each simulation (Figure $3.17 \mathrm{~b}$ ) shows a more prominent increase in the IQR and extremes between the steadily forced PiControl experiment to the Historical experiment and a 55.8\% drop in the IQR from Historical to the RCP2.6 experiment. From the Historical to RCP4.5, RCP6.0, and RCP8.5 there is a $44.7 \%, 44.4 \%$, and $40.2 \%$ drop in variability respectively. It is important to note that for the full records, the relative increase in RCP8.5 is only found between RCP4.5 and RCP6.0, overall RCP8.5 still displays smaller variability than the Historical. When the distributions for the full record are compared directly in the form of a histogram (Figure 3.18), the spread of 15-year trends for all six CMIP5 experiments is roughly the same interval between -0.4 to $0.4{ }^{\circ} \mathrm{C}$ per decade. We calculated the probability distributions here to better compare directly as each PiControl and RCP experiment had a fewer number of sampling points compared to Historical record. Overall, the six distributions display nearly normal distributions with a narrowing in the distribution of 15 -year periods for the full and both sub-setted periods (not shown) from the Historical experiment compared to the other five CMIP5 experiments. A decrease in the distribution suggests a decrease in natural variability characterized in this case as the probability of a large positive or negative 15-year trend in GMST, is likely as the total amount of GHG increase. The results from the time series 
analysis presented here are consistent with previous studies (Roberts et al., 2015; Schurer et al., 2015; Li and Baker, 2016; Rahmnstorf et al., 2017) that the possibility of a 15-year cooling period is to be expected despite the overall decrease in variability as forcing increases.

\subsubsection{Sea Surface Temperature}

While the IPCC report focused on surface air temperature for the computation of the hiatus, the dominant modes of variability driving the surface air temperature over timescales longer than a year are driven by variability in sea surface temperature (SST). Many studies have confirmed that for much of the globe, natural variability is driven by SSTs (Deser et al., 2010; Kosaka and Xie, 2013; Trenberth et al., 2014). Here, we ran the Monte Carlo sampling method using global annual mean SST to determine if the changes in variability seen in the previous section are also seen in the global oceans.

In Figure 3.19, we have again plotted the distribution of 15-year trends using a box and whisker plot over the entire linearly detrended multi-model concatenated global mean sea surface temperature (GMSST) time series for each of the six simulations. Using GMSST we incorporated all 19 CMIP5 models presented in Table 2.1. From the PiControl to Historical experiment there is an increase in variability in both the IQR by $51.9 \%$ and the extremes. The increased variability with amplified forcing in the Historical simulation was also captured in surface temperature. Comparing the Historical experiment to the four RCP experiments there is a steady decrease in variability with increased anthropogenic forcing by $1.7 \%$ to RCP2.6, $10.4 \%$ to RCP4.5, and $20.8 \%$ to RCP6.0. A $23.9 \%$ increase in SST variability is shown from the Historical to RCP8.5 
experiment in the IQR. For the RCP8.5 simulation the variability increases substantially by 56.5\% from RCP6.0 and displays the largest variability among all of the future warming scenarios. The increase in IQR from RCP6.0 to RCP8.5 may be a consequence of removing the linear trend in an experiment that takes the shape of a nonlinear warming scenario. By removing such a robust signal what may be left over is random high frequency noise increasing the variability of 15 -year trends. The increase in IQR found in RC8.5 was not the result in the detrended RCP8.5 ensemble of temperature at the surface as the overall variability was reduced when compared to the Historical and RCP2.6 (Figure 3.16). Therefore, the increase found in RCP8.5 may also be an indication of the possible slow changes to variability to SST associated with the oceanic heat capacity and also the large amounts of sea ice added to the ocean from rapid melting with increased radiative forcing.

To better understand the discrepancy between the air and sea surface temperature results for RCP8.5 and the role of detrending. We next look at the distribution in the first and latter half of the century (similar to Figure 3.17) to see if there are any similarities to the results found in the previous section. In Figure 3.20a there is an overall decrease in the IQRs to the four RCP simulations from the Historical experiment with the exception of RCP 2.6 with an increase by $1.2 \%$. The extremes between the four future scenarios are similar (in the first 50 years) with the exception of RCP4.5, which displays the smallest overall distribution to anthropogenically-forced experiments. In Figure 3.20b, the distributions of 15 -year trends in the four future warming scenarios are comparable to one another with deviations noted in their extremes and all four RCP experiments decreasing in the IQR from the Historical. Also, overall the distributions for the five 
experiments with amplified forcing the boxplots are smaller than when randomly sampled over the entire record. When comparing the CMIP5 experiments from their first half of the record to the latter half, all boxplots show an increase in IQR from the first half to the latter half of the century with the exception of RCP2.6 and RCP8.5 which decrease by $22.5 \%$ and $1.6 \%$, respectively. The decrease seen in the IQR for the latter half of the record is in concordance with the results from the previous section and indicates that on a warmer globe natural variability is expected to decrease less in the oceans while the hiatus-like trends are expected to occur with the continual addition of GHGs.

\subsection{Summary}

To summarize, in this chapter, Monte Carlo sampling was first used on the HadCRUT4 surface temperature record to determine the likelihood of reduced periods of warming super imposed within a long term warming trend. Given the last observed quiescent period lasted roughly 15 years from 1998 to 2012, this was the basis for using a 15-year trend in our analysis. When sampling over the observed GMST, the probability distribution of 15-year trends did not resemble a normal statistical Gaussian bell curve where the calculated sampled trends fall within one standard deviation of the mean. Instead, the probability distribution is negatively skewed at -0.491 with the median of the sampled 15-year trends higher than the calculated trend over the observed hiatus period. The higher median 15-year trends may result in the rejection of the null hypothesis where periods of quiescent warming are not an unlikely event under the influence of increased anthropogenic forcing. However, with a relatively limited amount of observed 
temperature data we synthetically modeled the observed record 10,000 times as an AR1 process and resampled the data again 10,000 times to elucidate the likely hood of hiatuslike trends in the future. When the distribution of 15-year trends over the AR1 modeled data was compared to distributions of 18 linearly detrended models under the CMIP5 Historical experiment it is found that superimposed reduced periods of warming in the surface temperature are still likely even in the presence of increased GHGs; confirming the results from previous studies (Easterling and Wehner, 2009; Roberts et al., 2015; Schurer et al., 2015; Li and Baker et al., 2016; Rahmnstorf et al.2017).

Next, a 15-year trend sample was taken from linearly detrended RCP2.6 and RCP8.5 warming experiments to give us a range of possible 15 -year trends between the most liberal and conservative future warming possibilities. $61 \%$ of the ensemble members displayed a decrease in variability with increased future GHGs from RCP2.6 to RCP8.5; confirming the possibility for the signal of internal variability becoming overwhelmed over time as amplified forcing begins to dominate. We further tested this theory by sub-setting the two RCP simulations by the first half of the record for each model and the latter half of the record. When comparing both halves for two future warming scenarios for temperature at the surface, a statistically significant drop in variability in the latter half of the record was exhibited for $67 \%$ of the model members experiencing RCP2.6 radiative forcing and $44 \%$ of model members under the RCP8.5 experiment. The increase in variability over the last part of the next century in RCP8.5 was surprising and may be a result of the different simulated mechanisms responsible for natural variability within the individual models. 
Subsequently, we looked at the 18-member models multi-model statistics for all six CMIP5 experiments. The inclusion for all four future warming scenarios helps compare how variability changes within simulations that contain transient forcing with differing amplitudes. The analysis of the multi-model statistics is useful in eliminating possible differences of simulated variability among the individual model members. The results of analysis on the multi-model time series confirms an overall decrease in variability noted within both the probability distributions with and without the linear trend removed. With the trend still within the modeled data, hiatus-like trends become less likely as the amount of radiative forcing increases by scenario. For the linearly detrended distributions, the extremes and IQR within the boxplots from RCP8.5 to both the Historical and RCP2.6 experiments also display a decrease. However, a statistically significant increase was also found in the IQR between RCP4.5 and RCP6.0 to RCP8.5. When comparing the sub-set of the multi-model ensemble between the first half of each individual to the multi-model ensemble of the latter half of the record in each ensemble, a similar result emerges with an increase in overall variability in RCP8.5 from RCP6.0. However evaluating the frequency of hiatus-like events between the multi-model ensembles for the CMIP5 experiments, the likelihood of a 15-year cooling period is found to decrease as the concentration of well-mixed GHGs increases.

With natural variability found to be driven by SSTs we chose to extend our analysis to resampling over GMSST. In the analysis of SSTs we resampled over a multimodel time series comprised of 19 model members for global annually averaged SST. The results were analogous to the surface temperature analysis with the probability of a hiatus-like trends found to be not unusual and expected to continue under aggressive 
anthropogenic forcing however become less likely as natural variability decreases in the future with the exception of RCP8.5 which saw an increase in variability compared to the other CMIP5 experiments and when analysis variability within surface air temperature. As previously argued, removing a linear trend may in fact be a too simplistic approach (Huang et al., 1998; Wu et al., 2007; Estrada and Perron, 2016; Lai and Yoon, 2018) for the statistical analysis and be the reason an increase in variability noted in RCP8.5. In Chapter 4, we take a more phenomenological approach and utilize more complex methodologies for trend removal to examine dominant modes of natural variability in the ocean within individual model members during both halves of the century to see if a particular mode of natural variability is dominating and to examine if and how the characteristics of the variance is going to change. 


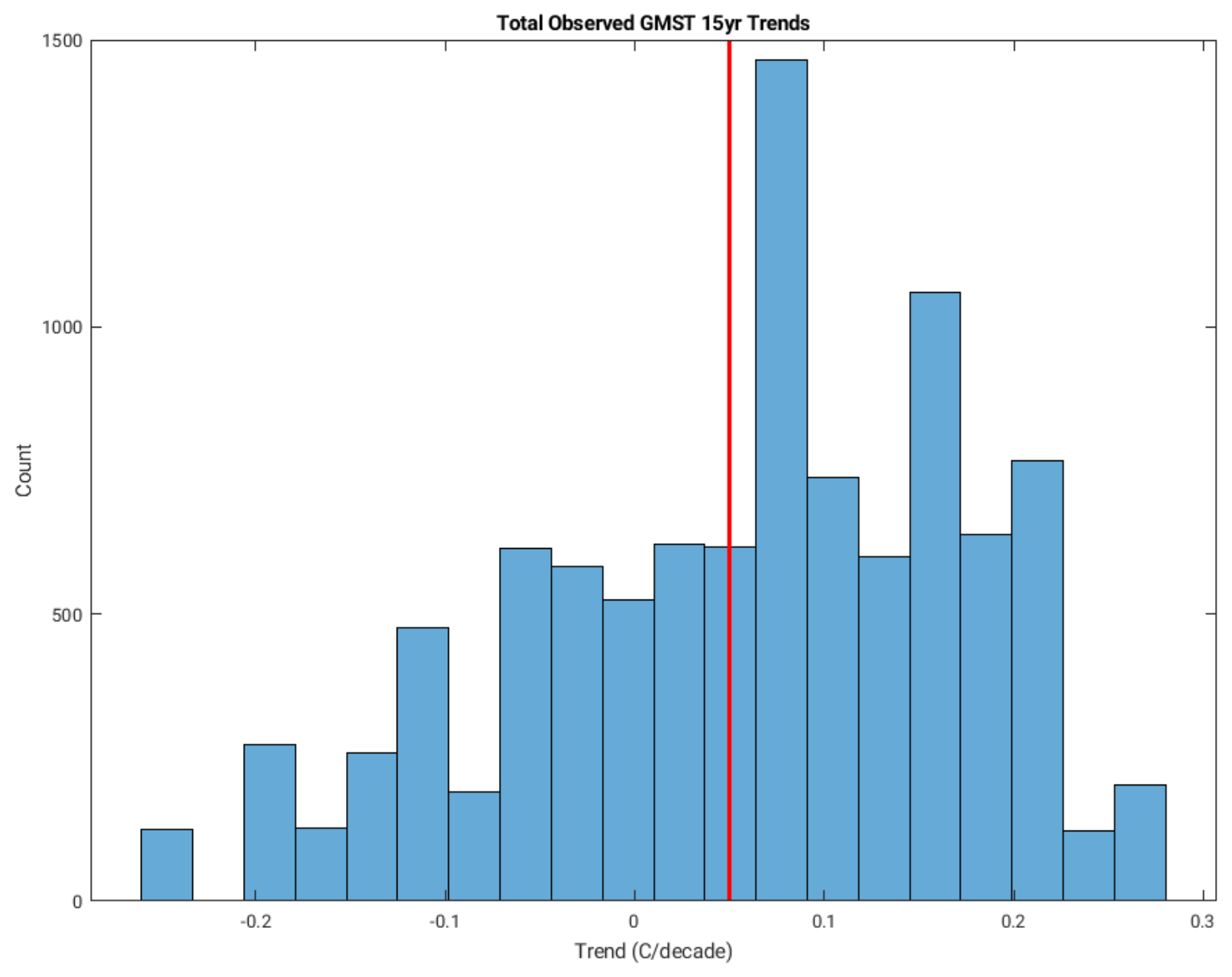

Figure 3.1: Histogram of the Monte Carlo sampling 10,000 times over 15-year trends from the observed HadCRUT4v global mean surface temperature time series from 18502017. The red line depicts the IPCC calculated trend over the hiatus period (1998-2012) of $0.05^{\circ} \mathrm{C}$ per decade. 


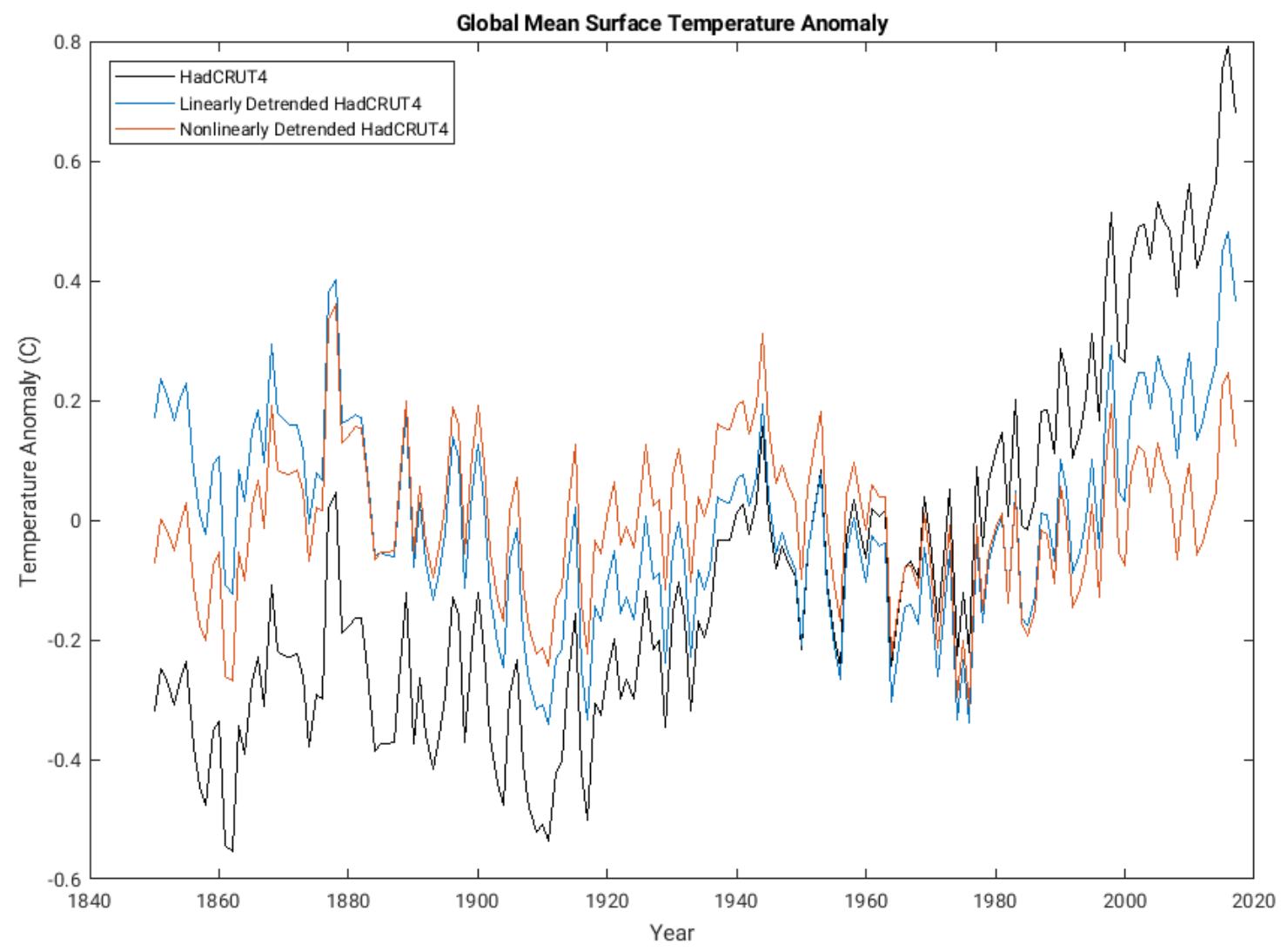

Figure 3.2: Observed annually averaged HadCRUT4 global mean surface temperature anomaly (black line) with the linearly detrended HadCRUT4 time series (blue line) and the nonlinearly detrended HadCRUT4 time series (orange line) from 1850-2017. 

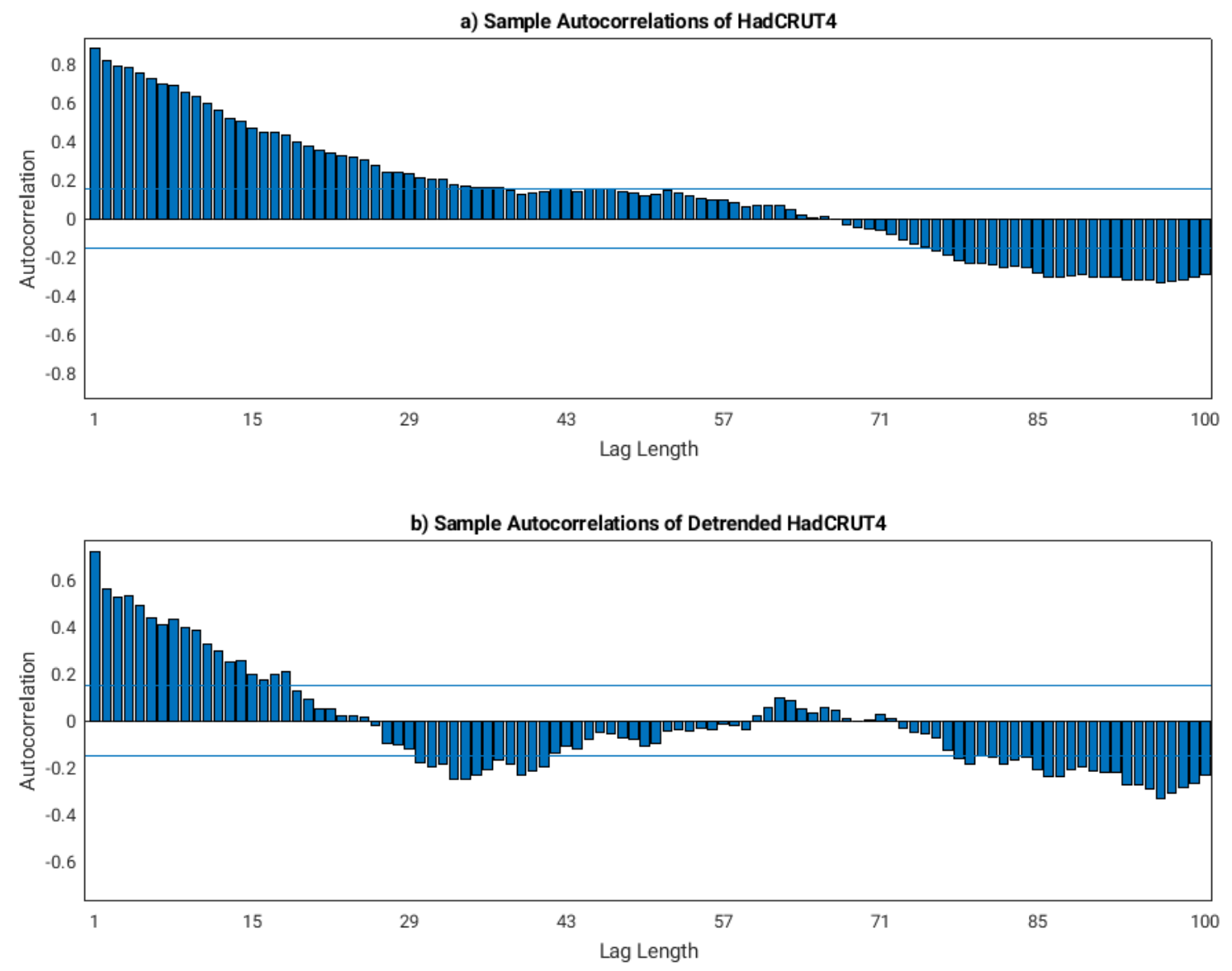

Figure 3.3: Autocorrelation Plot of the first 100 lags for a) observed GMST of HadCRUTv4 b) linearly detrended HadCRUT4v GMST between 1850-2017. 


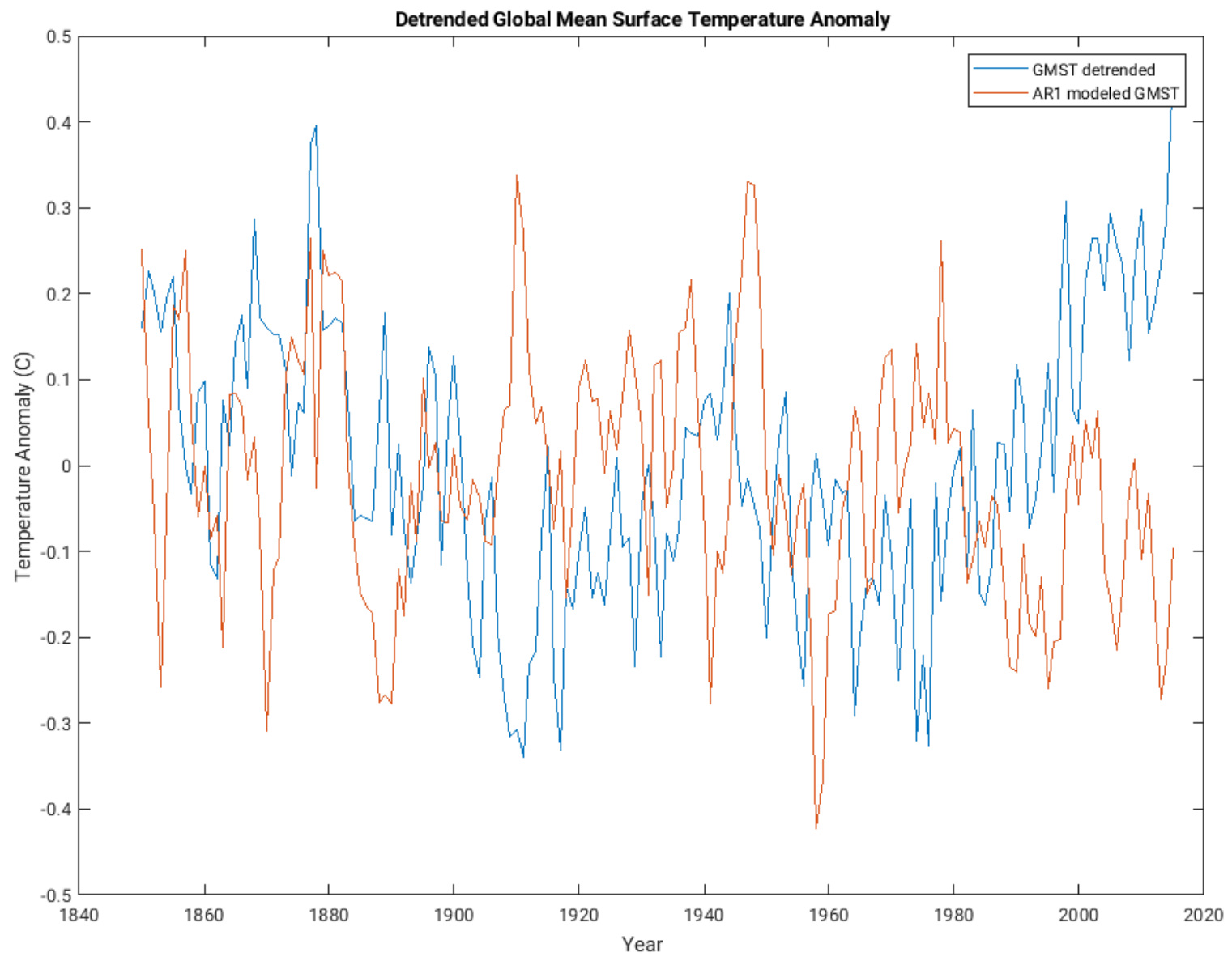

Figure 3.4: Time series of the linearly detrended annually averaged HadCRUT4 global mean surface temperature anomaly from 1850-2017 (blue curve) and one iteration from the AR1 modelled HadCRUT4 time series (chosen by the author; orange curve). 


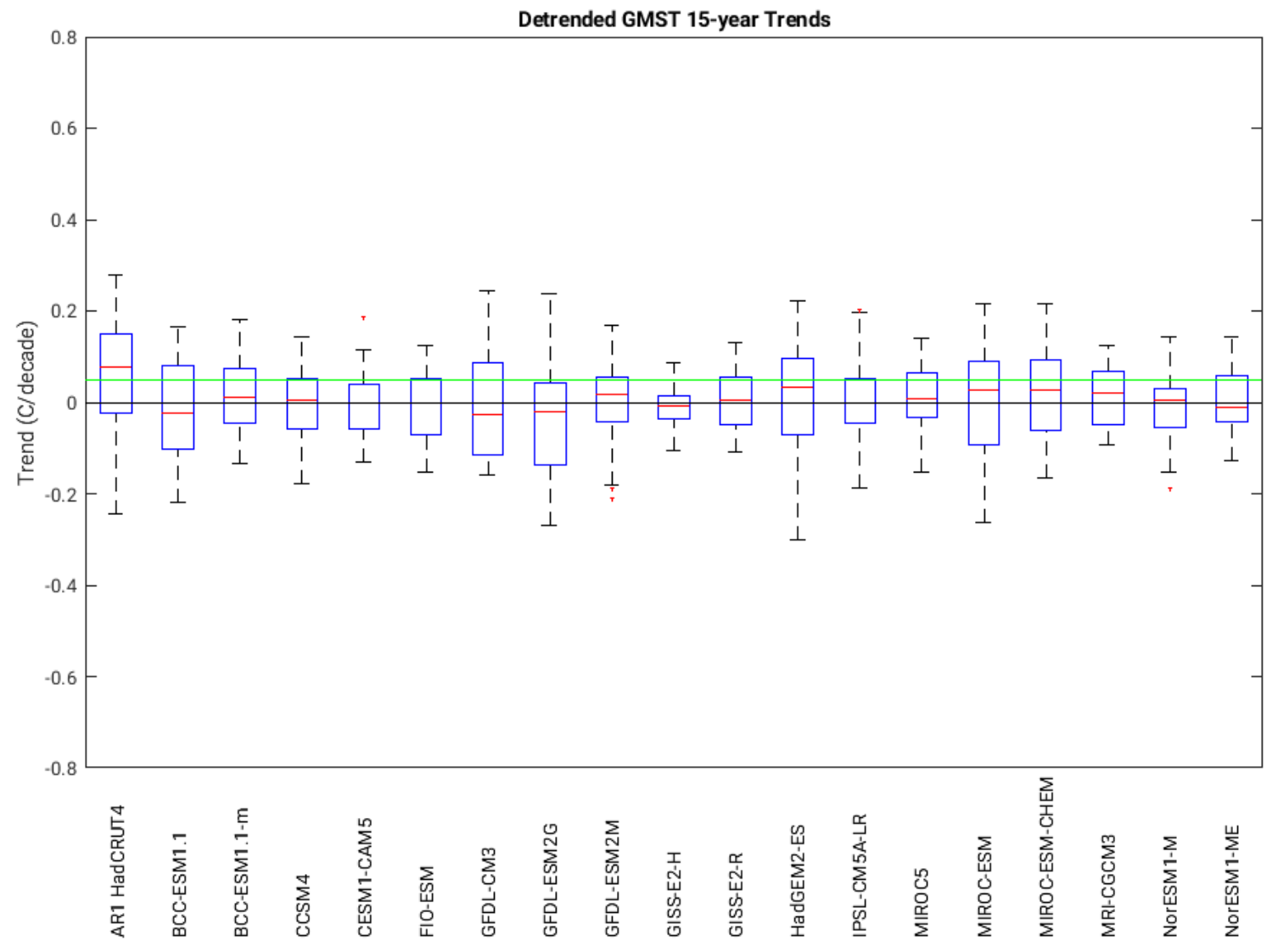

Figure 3.5: Box and whisker plots of the Monte Carlo sampled 15-year trends over 10,000 times from the HadCRUT4 GMST between 1850-2017 and the last 100 years for 18 CMIP5 models for the PiControl experiment which experience forcing at $280 \mathrm{ppm}$ throughout the entire simulation. The green line denotes the IPCC calculated trend of $0.05^{\circ} \mathrm{C}$ per decade over the hiatus period from 1998-2012. 


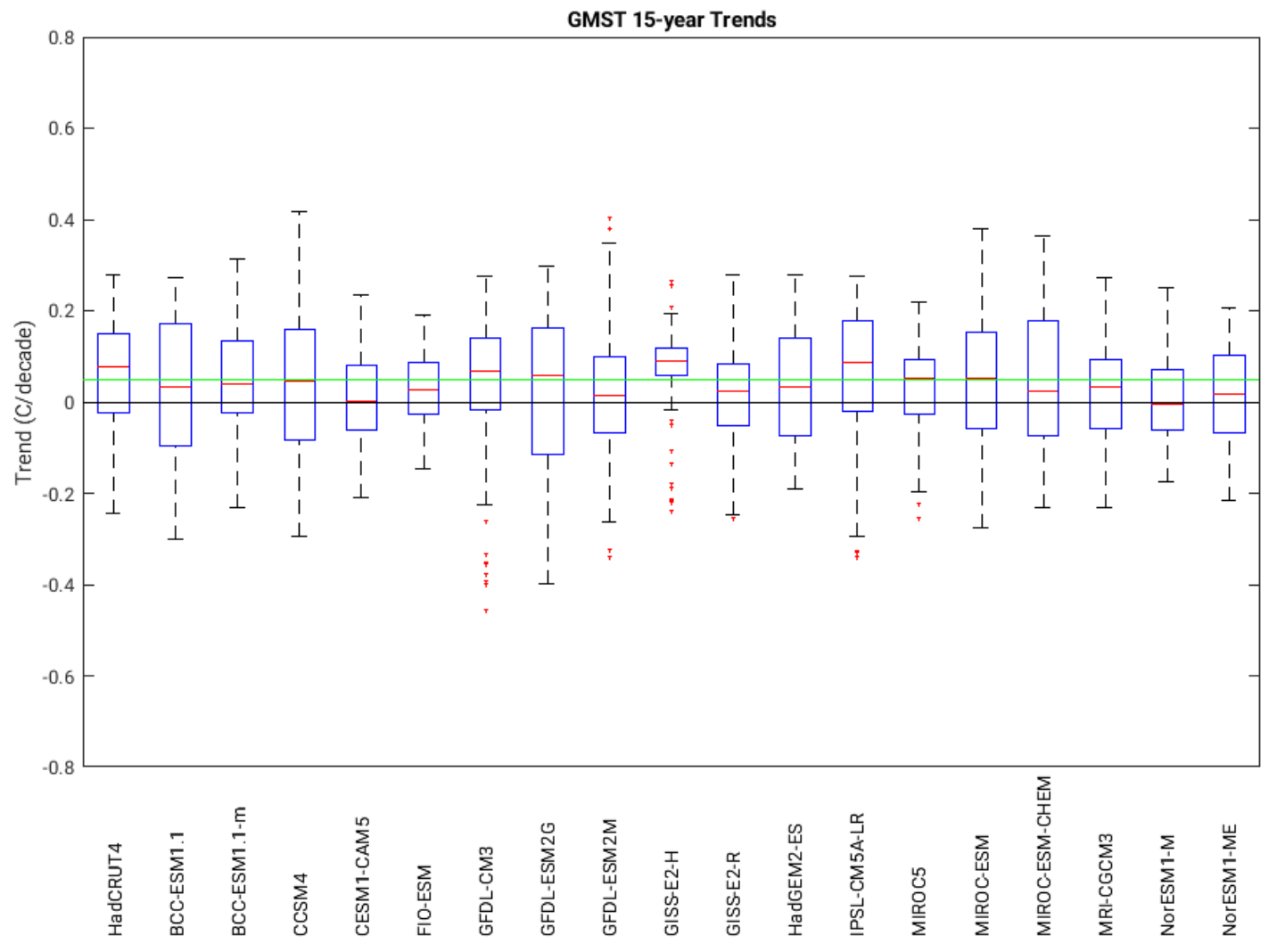

Figure 3.6: Box and whisker plots of the Monte Carlo sampled 15-year trends over 10,000 times from the HadCRUT4 GMST between 1850-2017 and 18 CMIP5 models for the Historical experiment from 1861-2005. The green line denotes the IPCC calculated trend of $0.05^{\circ} \mathrm{C}$ per decade over the hiatus period from 1998-2012. 


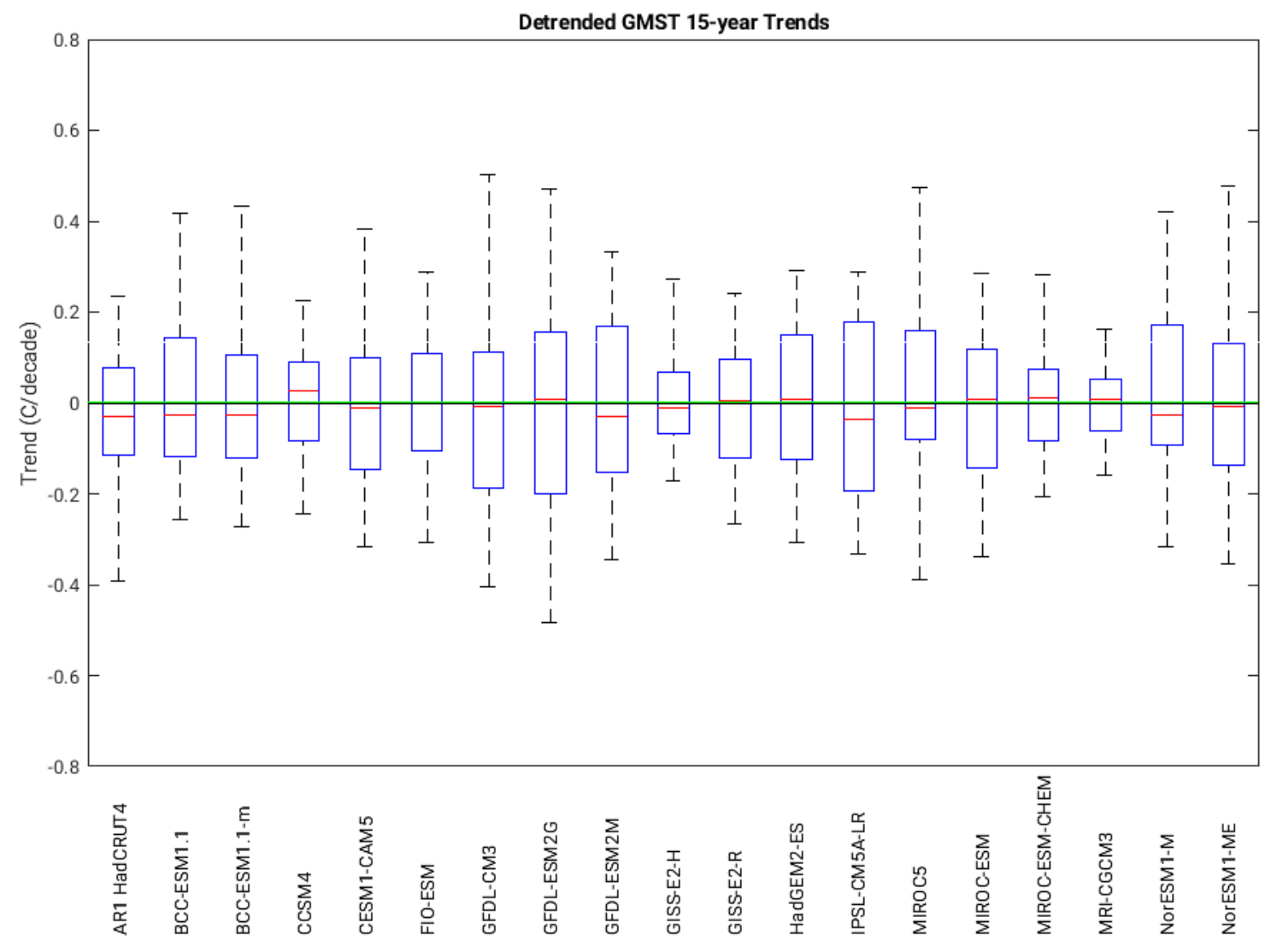

Figure 3.7: Box and whisker plots of the Monte Carlo sampled 15-year trends over 10,000 times from the AR1 simulated HadCRUT4 data between 1850-2017 and 18 AR1 simulated Historical models from 1861-2005. The green line denotes the detrended IPCC calculated trend of $0.002^{\circ} \mathrm{C}$ per decade over the hiatus period from 1998-2012. 


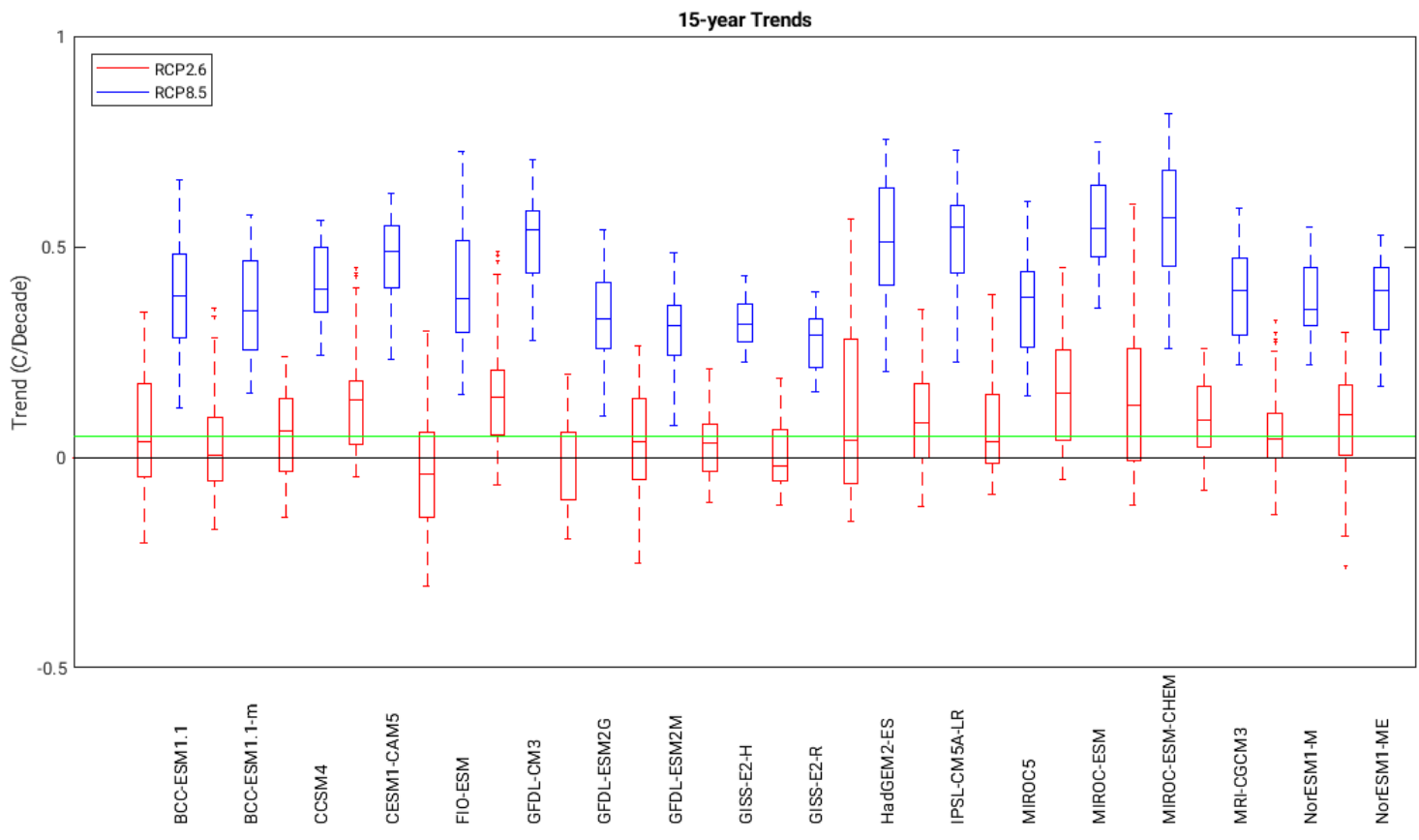

Figure 3.8: Box and whisker plots of the Monte Carlo sampled 15-year trends over 10,000 times for 18 CMIP5 models under the RCP2.6 experiment (2006-2100) in red and the RCP8.5 experiment (2006-2100) in blue. The green line denotes the IPCC calculated trend of $0.05^{\circ} \mathrm{C}$ per decade over the hiatus period from 1998-2012. 


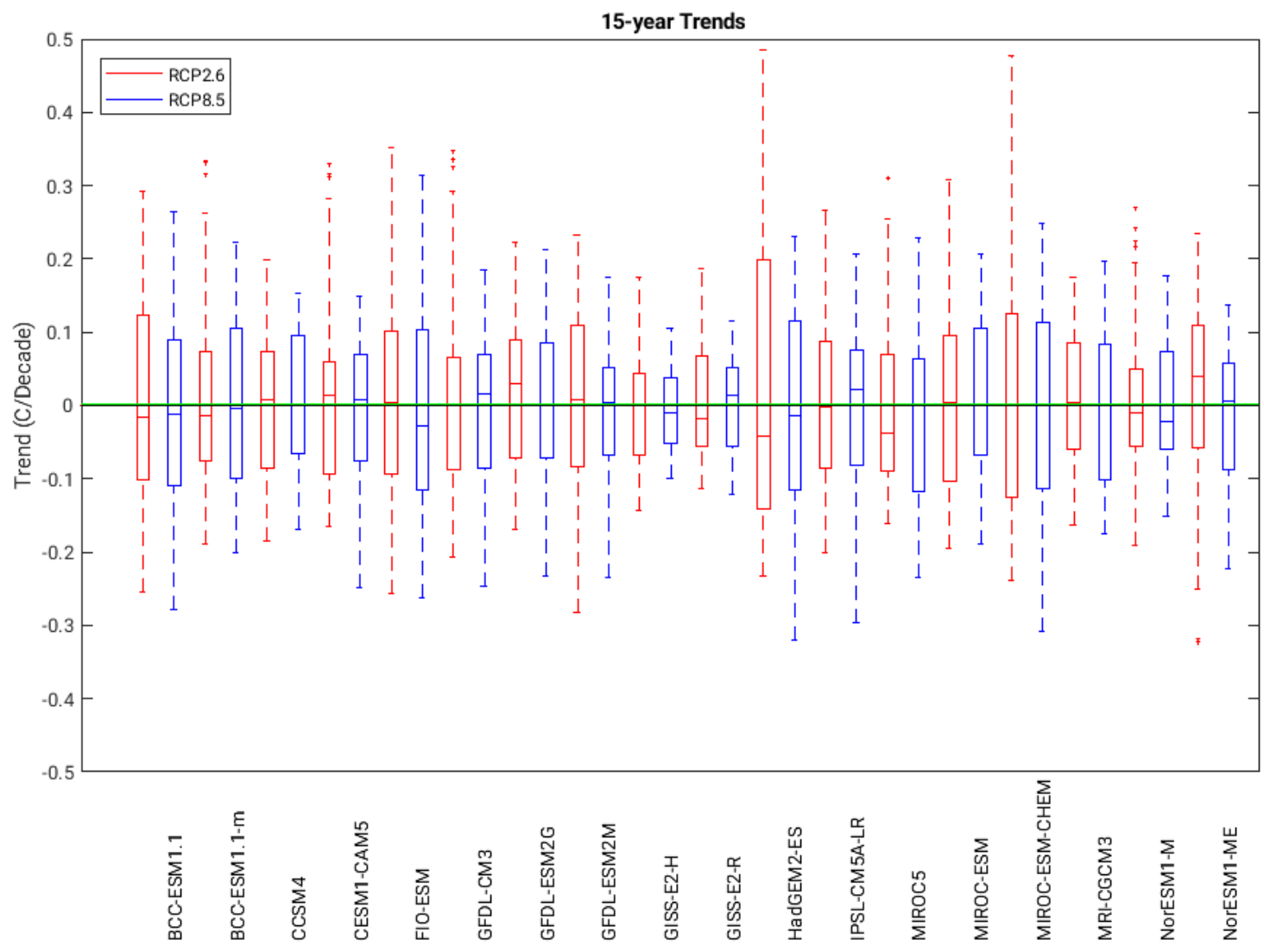

Figure 3.9: Box and whisker plots of the Monte Carlo sampled 15-year trends over 10,000 times for 18 linearly detrended CMIP5 models under the RCP2.6 experiment (2006-2100) in red and the RCP8.5 experiment (2006-2100) in blue. The green line denotes the linearly detrended IPCC calculated trend of $0.002^{\circ} \mathrm{C}$ per decade over the hiatus period from 1998-2012. 


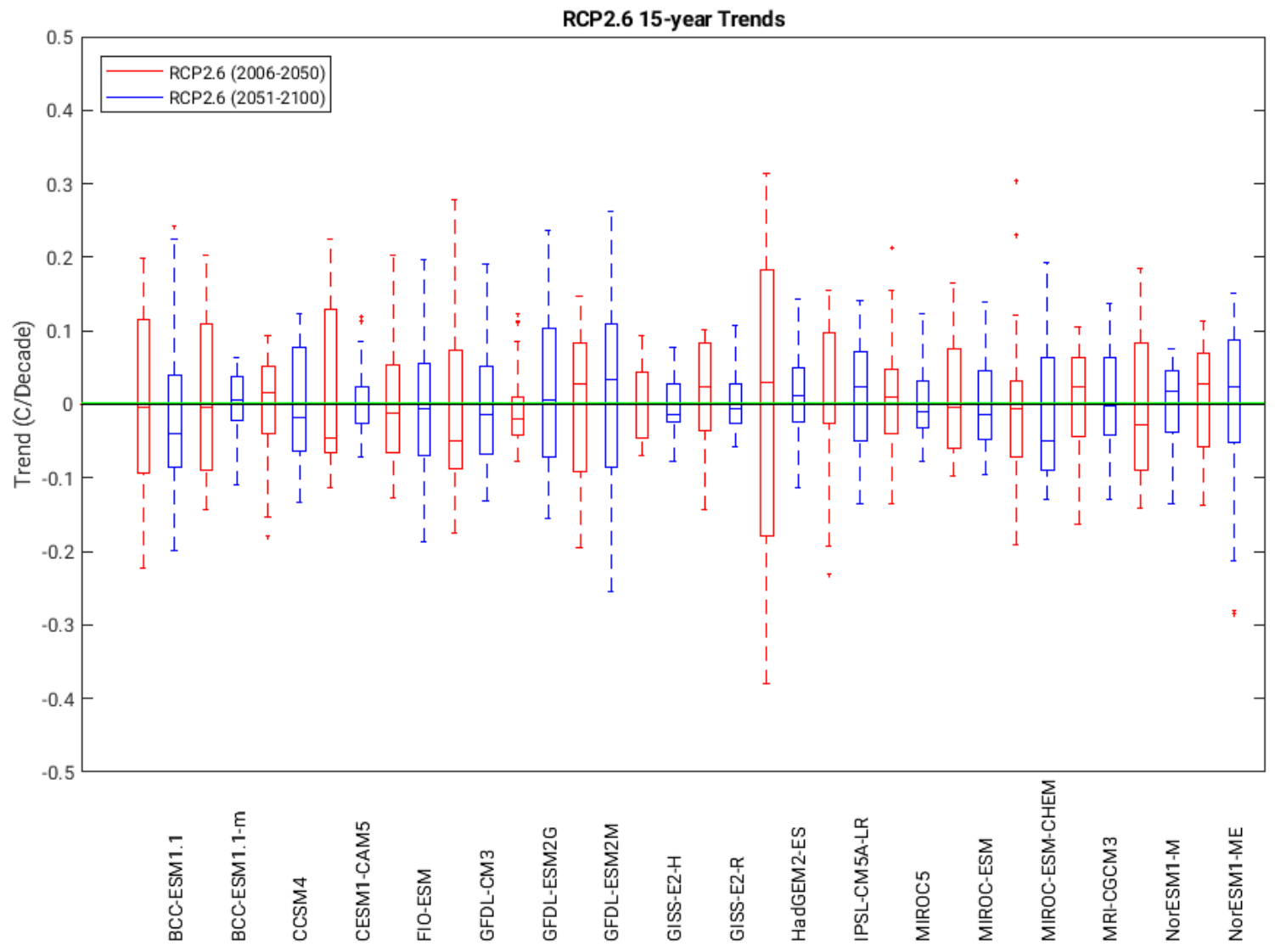

Figure 3.10: Box and whisker plots of the Monte Carlo sampled 15-year trends over 10,000 times for 18 linearly detrended models under the RCP2.6 experiment. Red boxes are the Monte Carlo sampled 15-year trends calculated over the modeled years, 20062050 in each model member and blue boxes are the 15-year trends sampled over the modeled years 2051-2100 in each member. The green line denotes the linearly detrended IPCC calculated trend of $0.002^{\circ} \mathrm{C}$ per decade over the hiatus period from 1998-2012. 


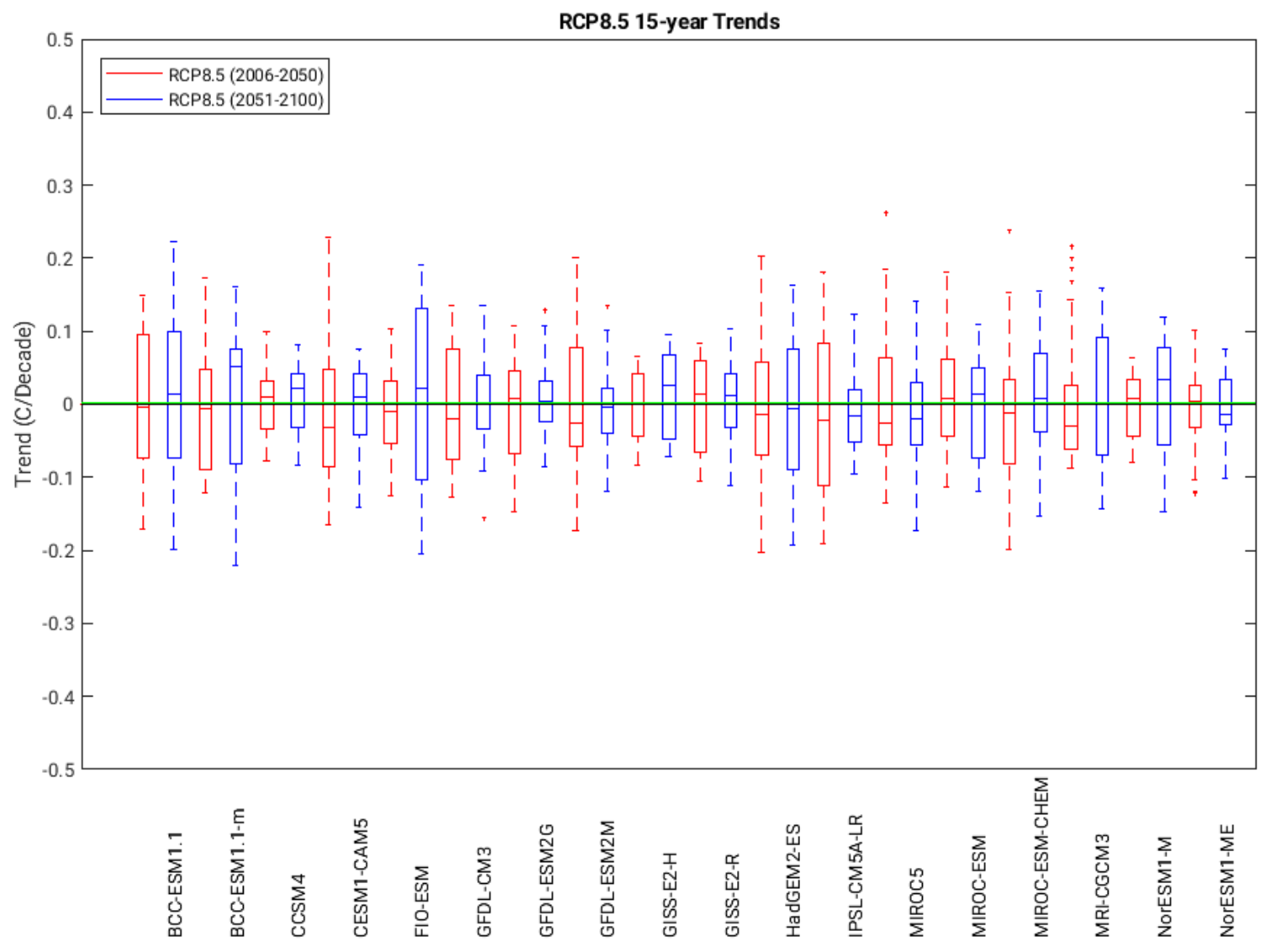

Figure 3.11: Box and whisker plots of the Monte Carlo sampled 15-year trends over 10,000 times for 18 linearly detrended models under the RCP8.5 experiment. Red boxes are the Monte Carlo sampled 15-year trends calculated over the modeled years, 20062050 in each model member and blue boxes are the 15-year trends sampled over the modeled years 2051-2100 in each member. The green line denotes the linearly detrended IPCC calculated trend of $0.002^{\circ} \mathrm{C}$ per decade over the hiatus period from 1998-2012. 

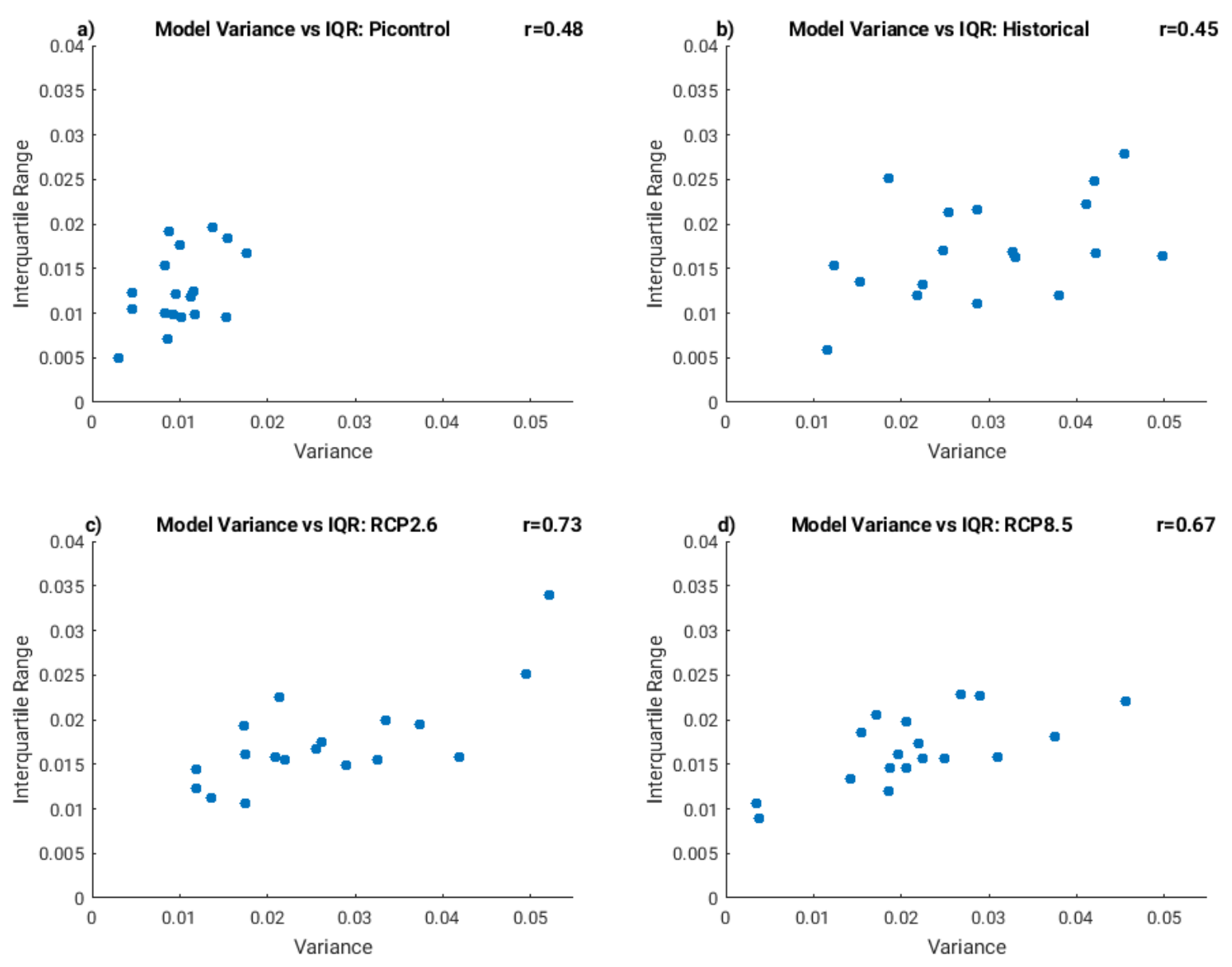

Figure 3.12: Scatter plots of the model variance versus interquartile range of the Monte Carlo sampled 15-year trends over 10,000 times before linearly detrending for 18 model members under the a) PiControl (last 100 years in each model), b) Historical (18612005), d) RCP2.6 (2006-2100) and d) RCP8.5 (2006-2100) experiment. 

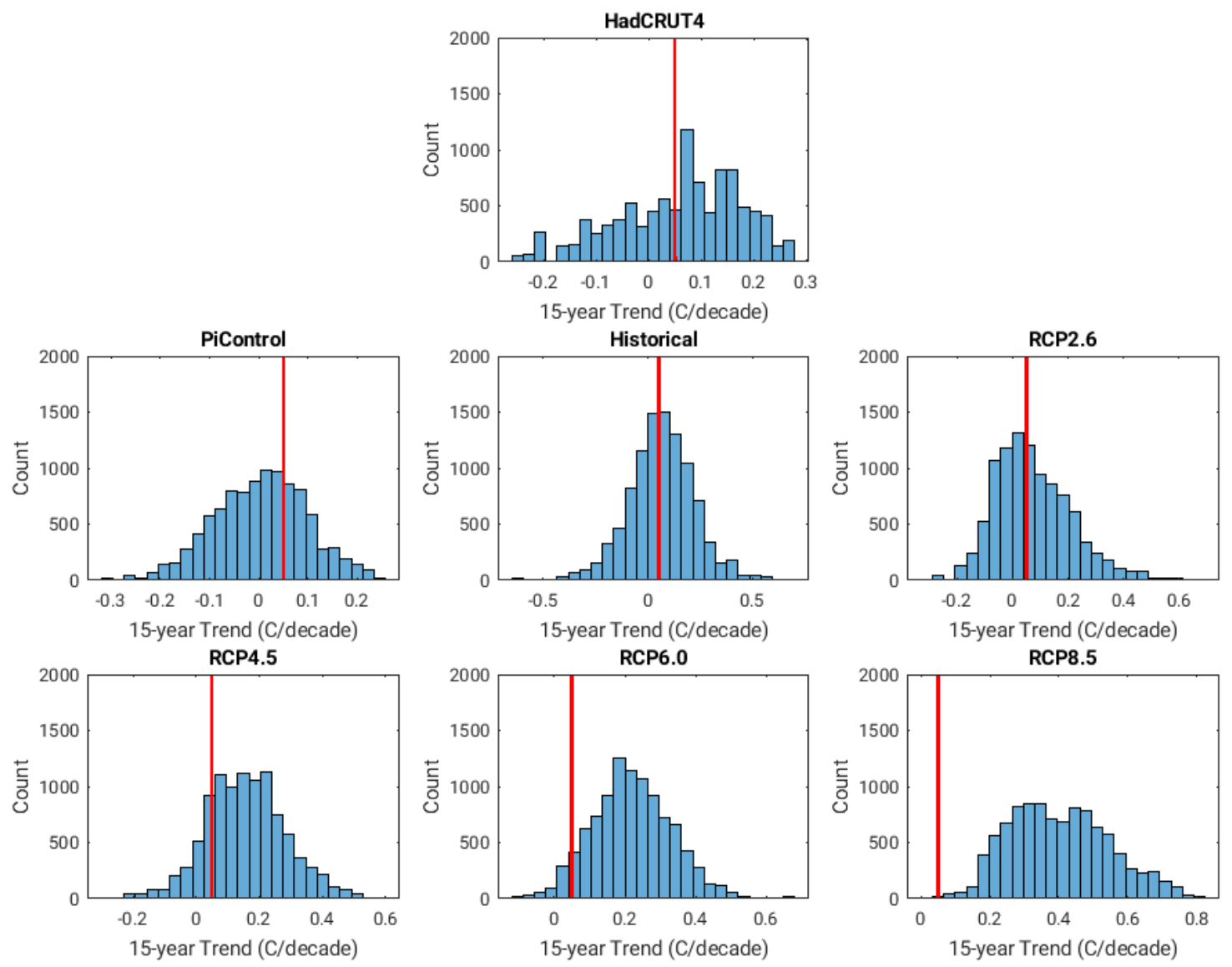

Figure 3.13: Distribution of the Monte Carlo sampled 15-year trends over 10,000 times over the a) observed HadCRUT4 time series (1850-2017) and for the multi-model GMST time series concatenated for the full record of each model member for experiments $b$ ) PiControl, c) Historical, d) RCP2.6, e) RCP4.5, f) RCP6.0, g) RCP8.5. The red line depicts the trend calculated over the recent hiatus (1998-2012) in HadCRUT4. Combining the 18-member ensemble gives us 1800 simulated years to sample over the last 100 years in each PiControl experiment, 2610 modeled years in the Historical experiment from 1861-2005, and 1710 modeled years for all four RCP experiments between 2006-2100. 

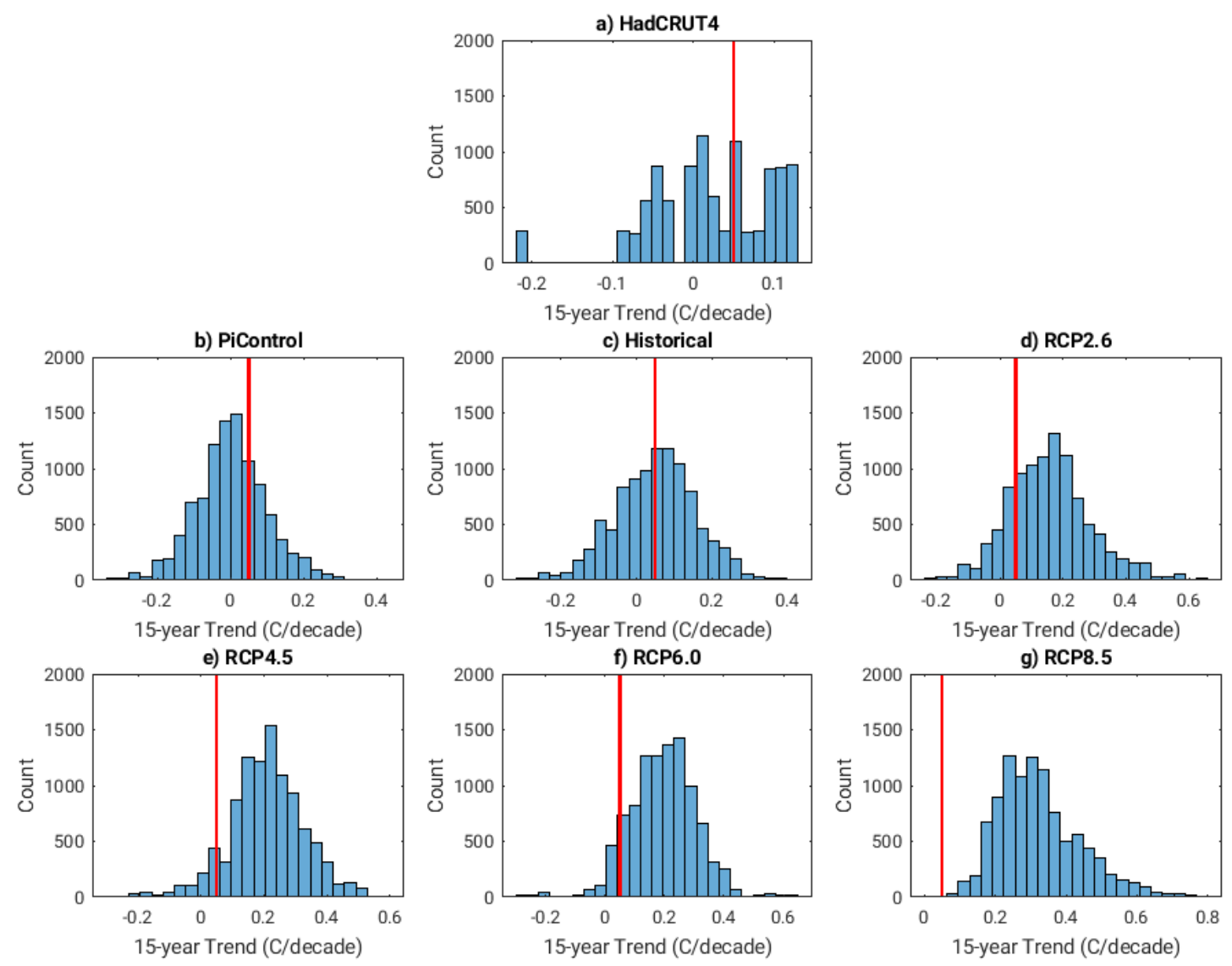

Figure 3.14: Distribution of the Monte Carlo sampled 15-year trends over 10,000 times over the first 50 years (1900-1950) for the a) observed HadCRUT4 time series and for the multi-model GMST time series concatenated with the first 50 years of each model member for CMIP5 experiments b) PiControl, c) Historical, d) RCP2.6, e) RCP4.5, f) RCP6.0, g) RCP8.5. The red line depicts the trend calculated over the recent hiatus (1998-2012) in HadCRUT4. Combining the 18-member ensemble gives us 900 simulated years to sample over the first years in each PiControl experiment and the Historical experiment from 1900-1950, and 792 modeled years for all four RCP experiments between 2006-2050. 

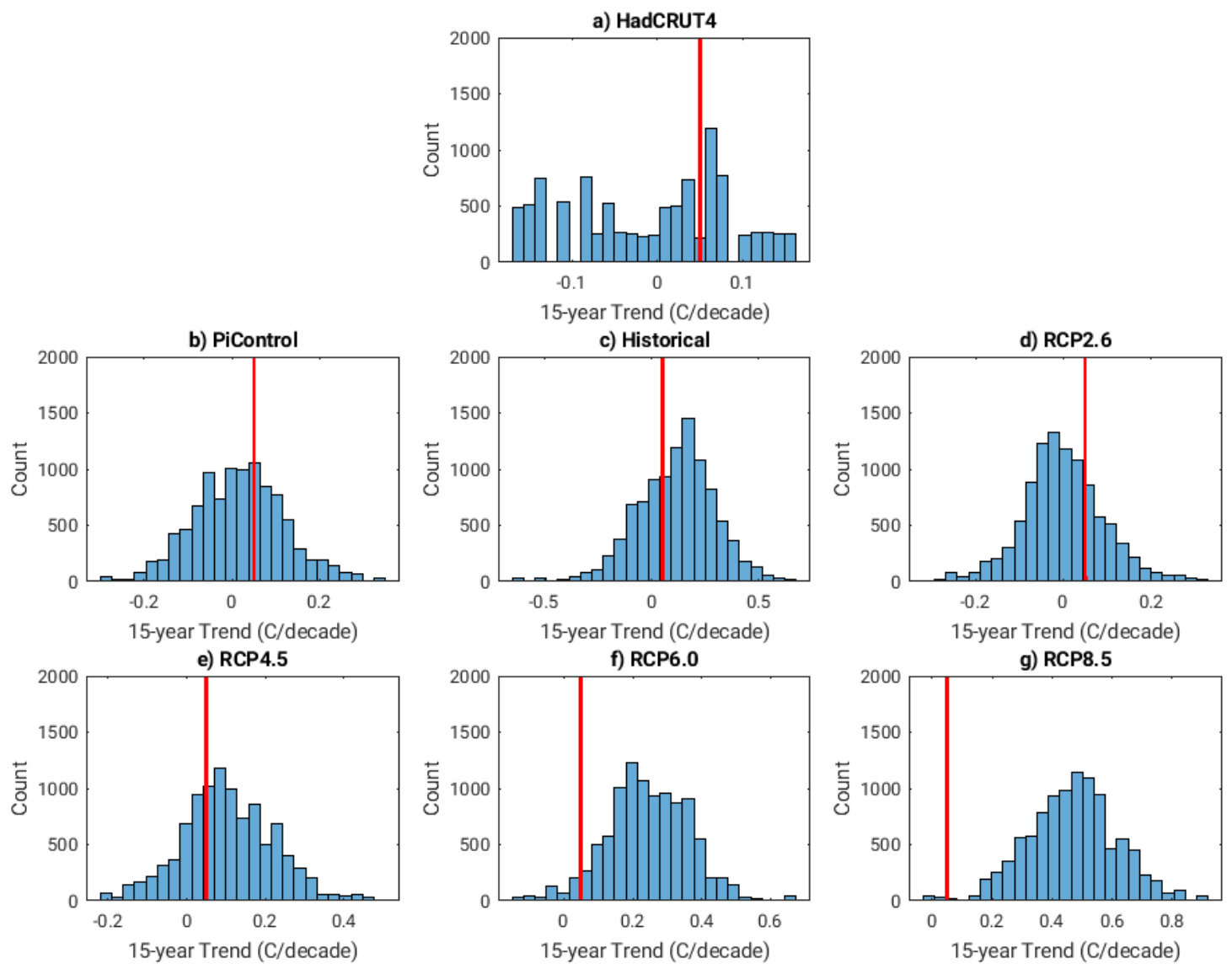

Figure 3.15: Distribution of the Monte Carlo sampled 15-year trends over 10,000 times over the last 50 years (1951-2017) over the a) observed HadCRUT4 time series and for the multi-model GMST time series concatenated with the last 50 years of each model member for CMIP5 experiments b) PiControl, c) Historical, d) RCP2.6, e) RCP4.5, f) RCP6.0, g) RCP8.5. The red line depicts the trend calculated over the recent hiatus (1998-2012) in HadCRUT4. Combining the 18-member ensemble gives us 900 simulated years to sample over the last 50 years in each PiControl experiment, 972 simulated years for the Historical experiment from 1951-2005, and 900 modeled years for all four RCP experiments between 2051-2100. 


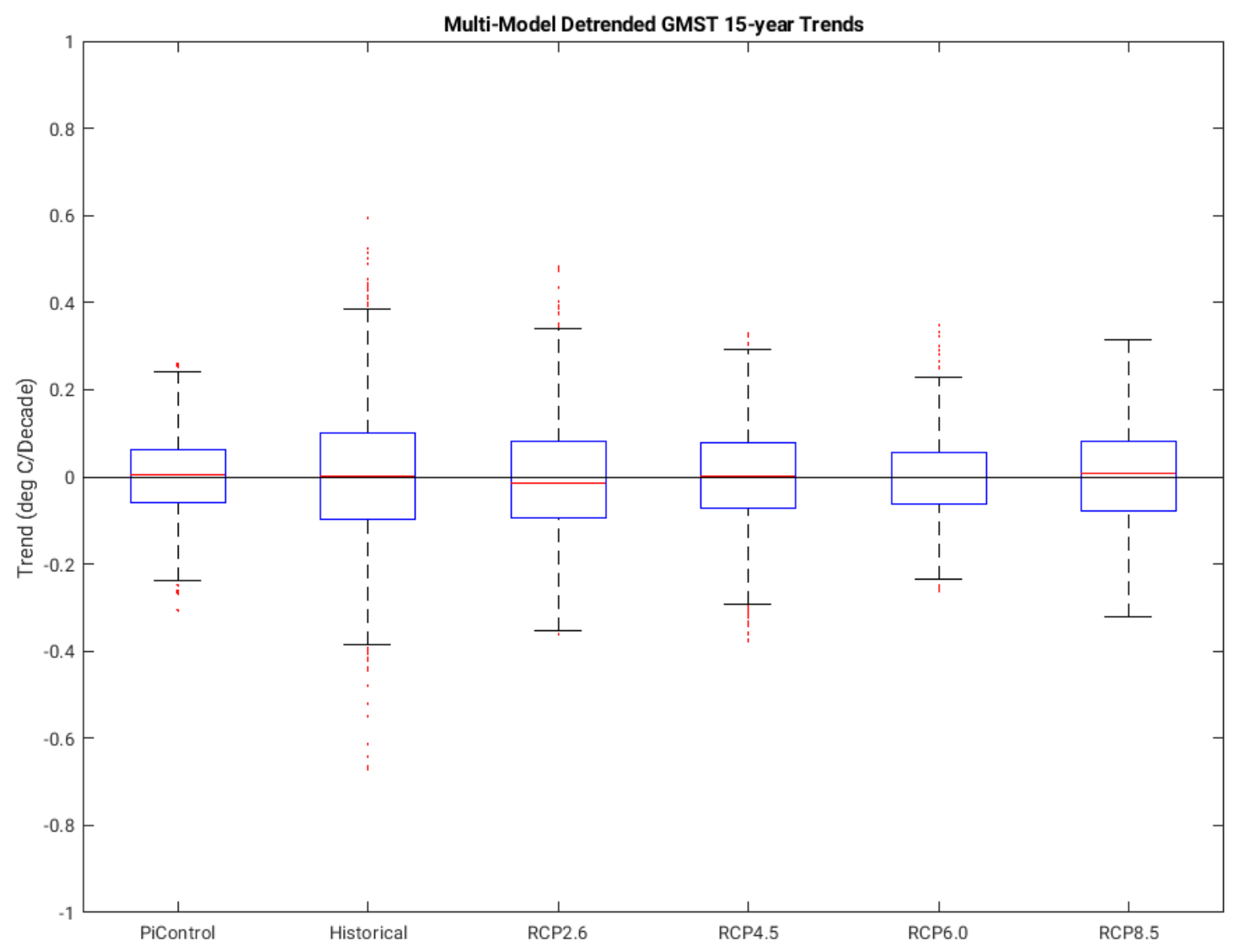

Figure 3.16: Box and whiskers plot of the Monte Carlo sampled 15-year trends over 10,000 times for the multi-model linearly detrended time series concatenated using the full GMST for the PiControl, Historical, RCP2.6, RCP4.5, RCP6.0, and RCP8.5. Combining the 18-member ensemble over the full record results in 1800 simulated years to sample over the last 100 years in each PiControl experiment, 2610 modeled years in the Historical experiment from 1861-2005, and 1710 modeled years for all four RCP experiments between 2006-2100. 


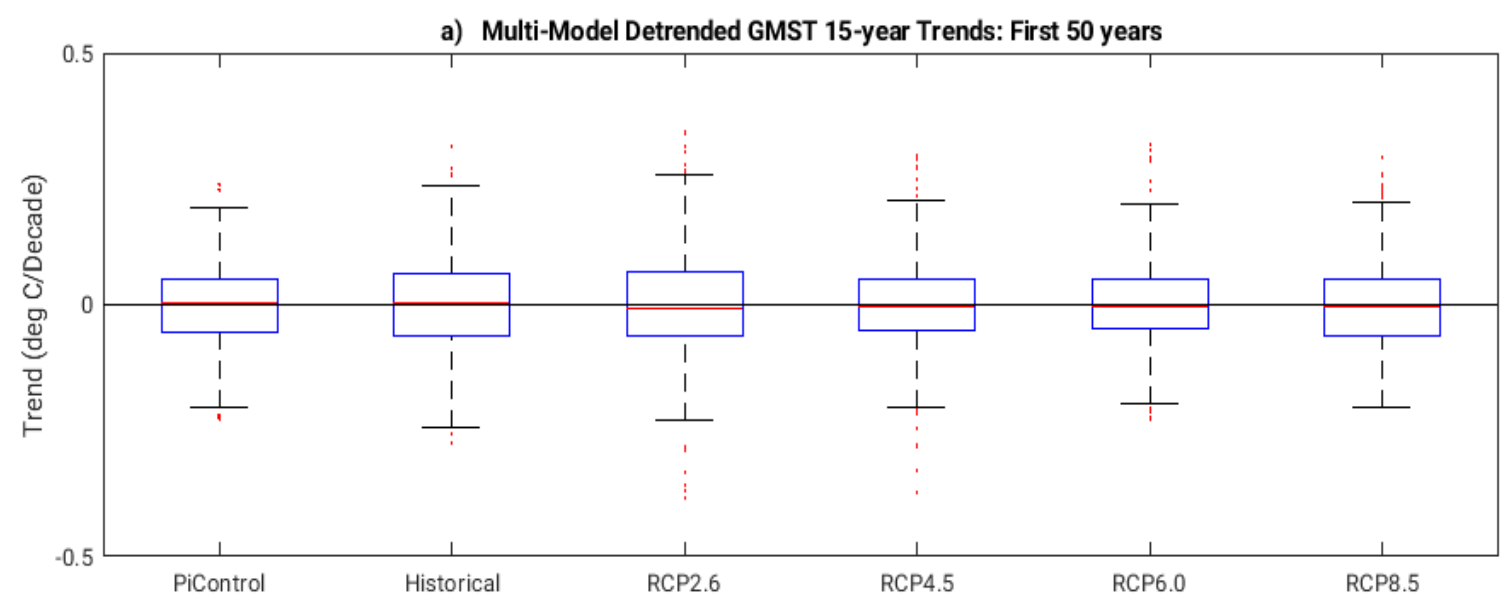

b) Multi-Model Detrended GMST 15-year Trends: Last 50 years

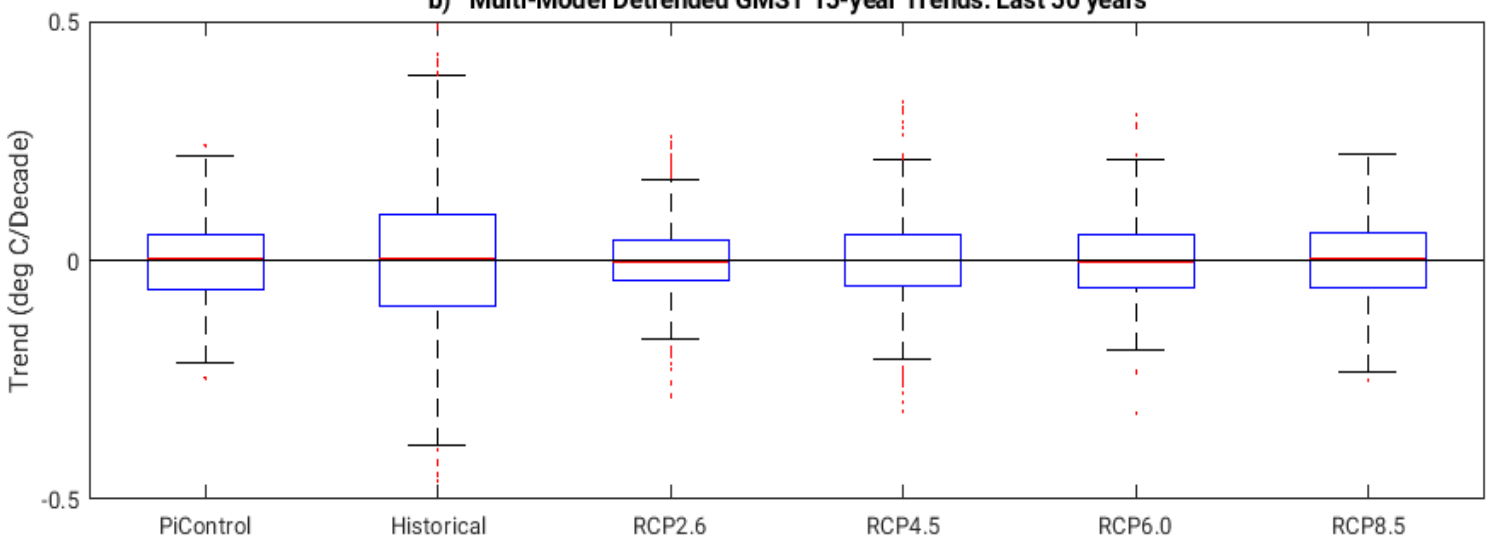

Figure 3.17: Box and whisker plots of the Monte Carlo sampled 15-year trends over 10,000 times from the multi-model linearly detrended time series concatenated with a) the first 50 years of each ensemble member and b) the last 50 years of each ensemble member for the PiControl, Historical, RCP2.6, RCP4.5, RCP6.0, and RCP8.5 experiments. Combining the 18-member ensemble over the first years produces 900 simulated years to sample in each PiControl experiment, 972 simulated years for the Historical experiment from 1900-1950, and 900 modeled years for all four RCP experiments between 2006-2050. While combining over the last half of each model member produces 900 simulated years to sample over the last 50 years in each PiControl experiment, 972 simulated years for the Historical experiment from 1951-2005, and 900 modeled years for all four RCP experiments between 2051-2100. 


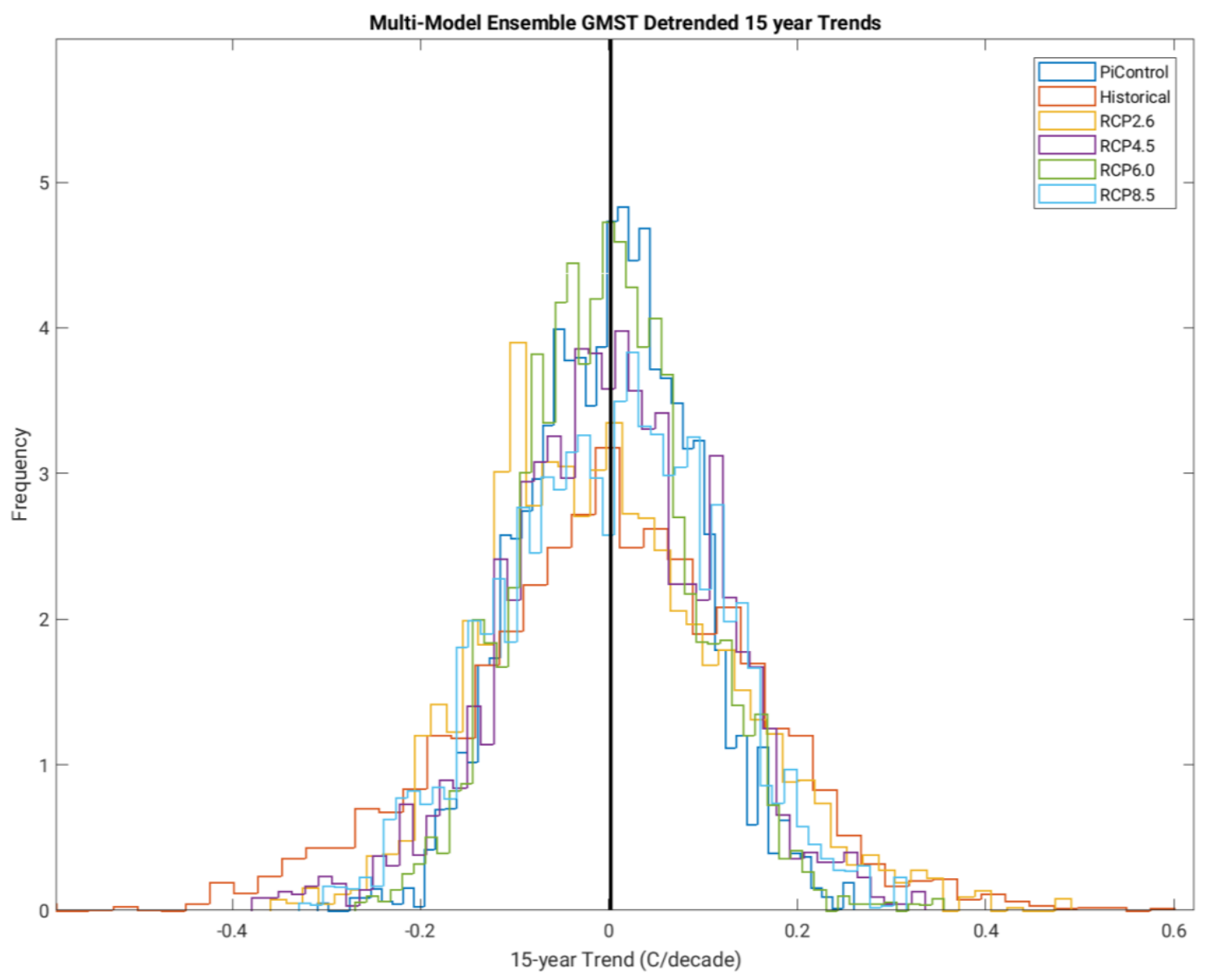

Figure 3.18: Normalized histogram of the Monte Carlo sampled 15-year trends over 10,000 times for the linearly detrended full record of the multi-model time series for the PiControl, Historical, RCP2.6, RCP4.5, RCP6.0, and RCP8.5. Combining the 18-member ensemble results in 1800 simulated years to sample over the last 100 years in each PiControl experiment, 2610 simulated years in the Historical experiment from 18612005, and 1710 modeled years for all four RCP experiments between 2006-2100. The black line depicts the trend calculated over the recent hiatus (1998-2012) in HadCRUT4. 


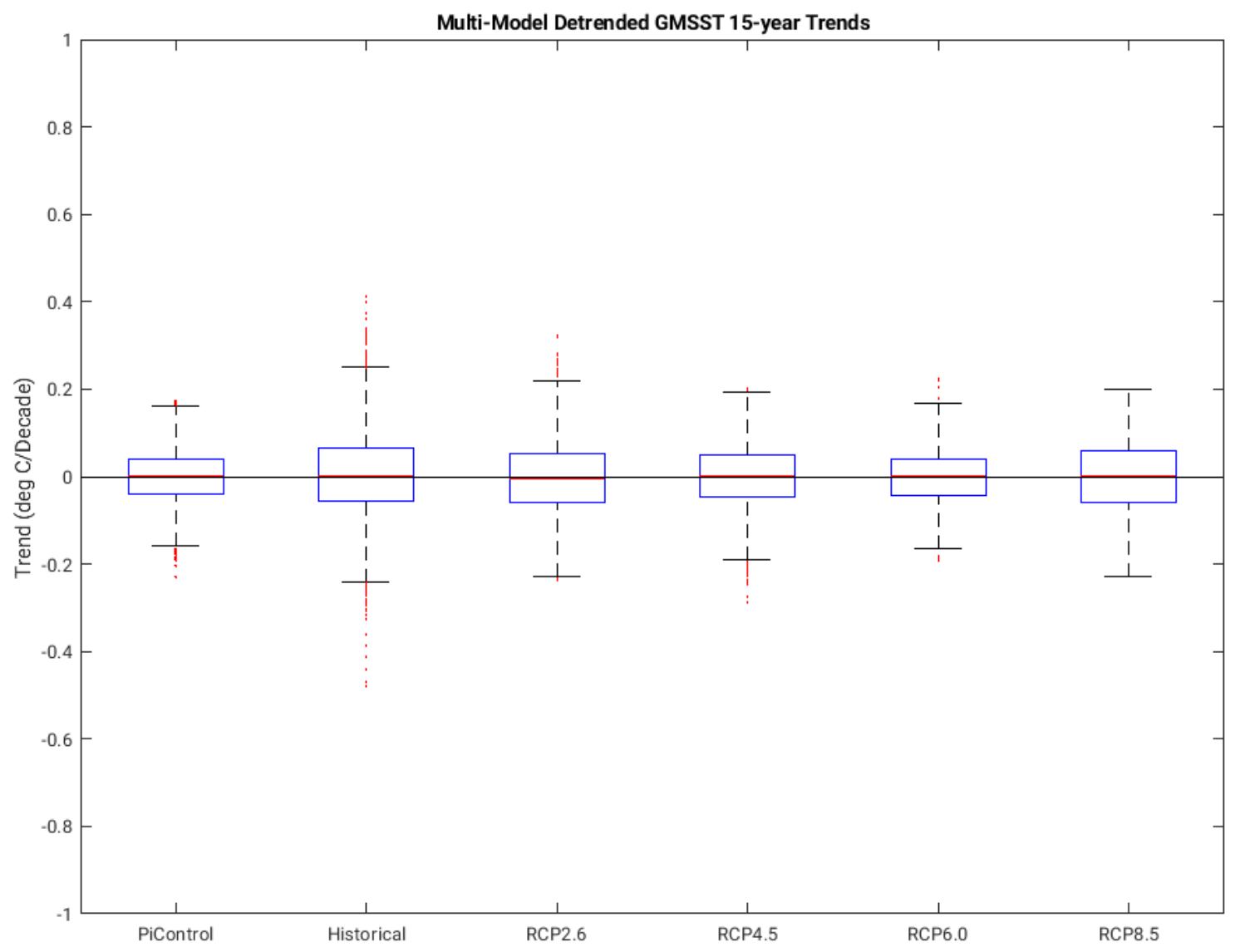

Figure 3.19: Box and whiskers plot of the Monte Carlo sampled 15-year trends over 10,000 times for the multi-model linearly detrended time series concatenated using the full global mean sea surface temperature for the PiControl, Historical, RCP2.6, RCP4.5, RCP6.0, and RCP8.5. Combining the 18-member ensemble over the full record results in 1800 simulated years to sample over the last 100 years in each PiControl experiment, 2610 modeled years in the Historical experiment from 1861-2005, and 1710 modeled years for all four RCP experiments between 2006-2100. 


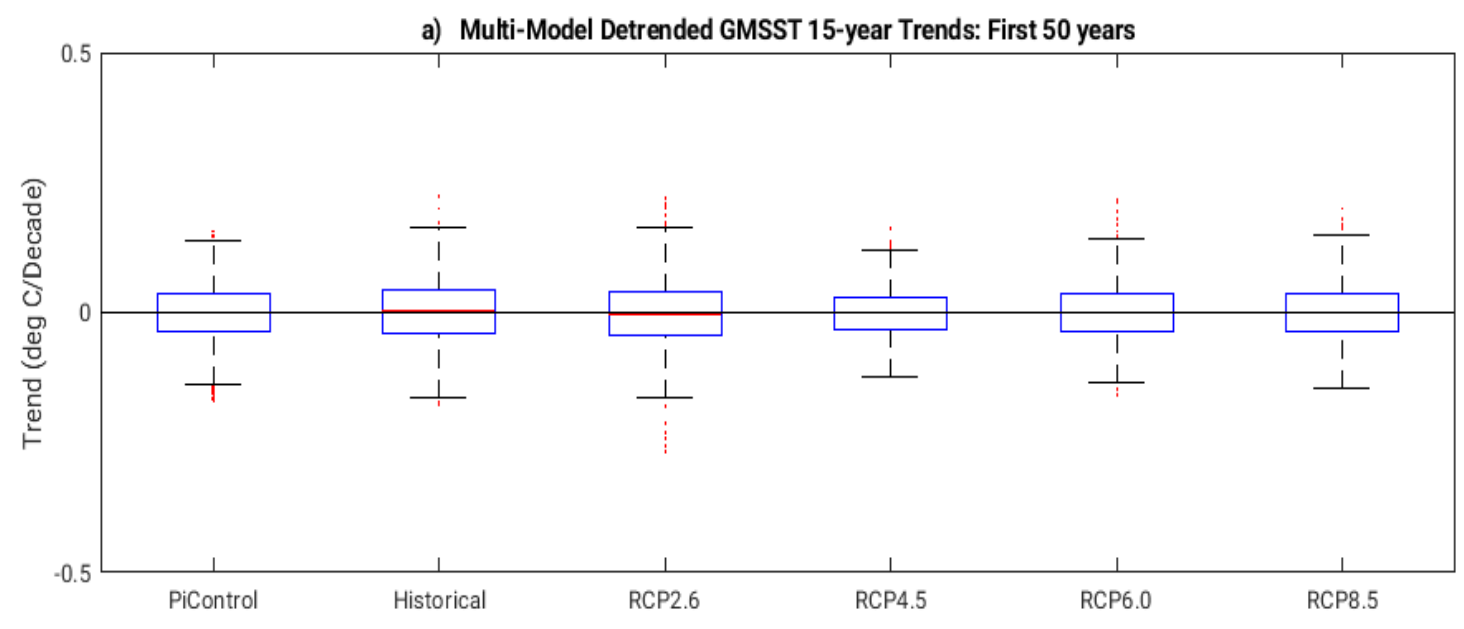

b) Multi-Model Detrended GMSST 15-year Trends: Last 50 years

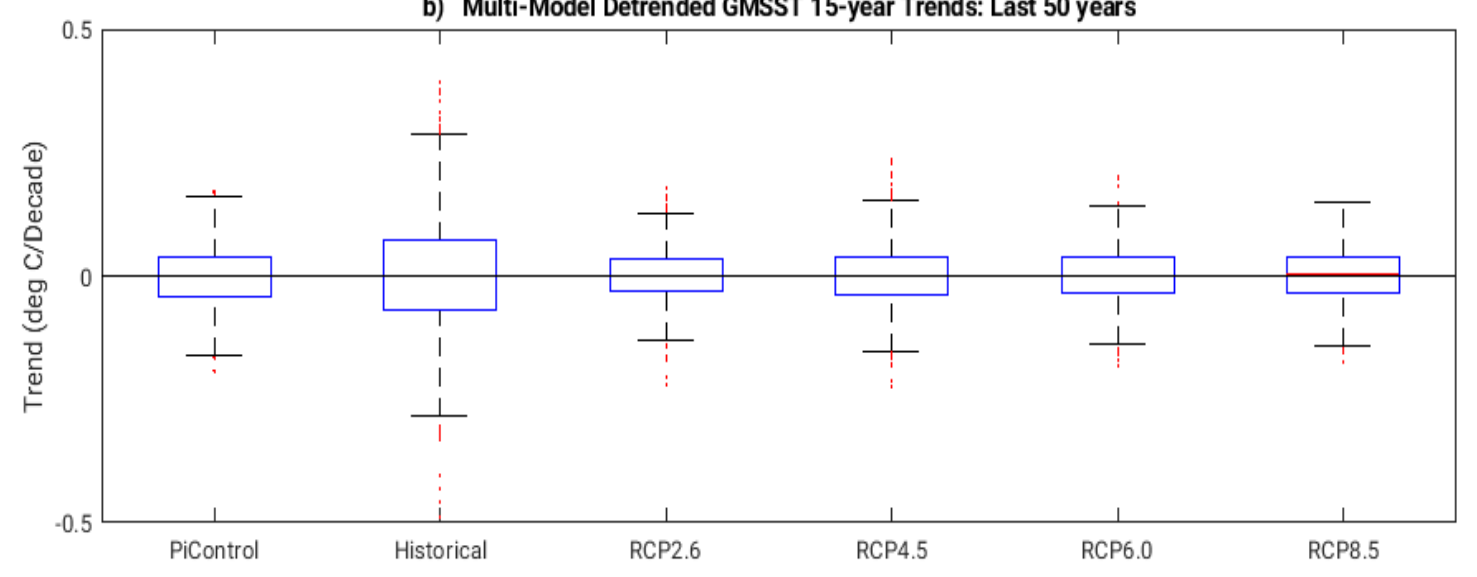

Figure 3.20: Box and whisker plots of the Monte Carlo sampled 15-year trends over 10,000 times from the multi-model linearly detrended global mean sea surface temperature time series concatenated with a) the first 50 years of each ensemble member and b) the last 50 years of each ensemble member for the PiControl, Historical, RCP2.6, RCP4.5, RCP6.0, and RCP8.5 experiments. Combining the 18-member ensemble over the first 50 years produces 900 simulated years to sample in each PiControl experiment, 972 simulated years for the Historical experiment from 1900-1950, and 900 modeled years for all four RCP experiments between 2006-2050. While combining over the last half of each model member produces 900 simulated years to sample over the last 50 years in each PiControl experiment, 972 simulated years for the Historical experiment from 1951-2005, and 900 modeled years for all four RCP experiments between 2051-2100. 


\section{CHAPTER 4: RESPONSE OF VARIANCE IN THE SEA SURFACE \\ TEMPERATURE AS A RESULT TO INCREASES IN RADIATIVE FOCING}

\subsection{Overview}

As we demonstrated in Chapter 3, the probability for a period of reduced warming comparable to the recent hiatus is not uncommon even on a warmer globe. Our analysis showed that natural variability might become less prominent in warmer scenarios. We also showed increased variance in the RCP8.5 scenario pointing to an as yet unidentified issue in the analysis. We think it lies in the simplicity of the analysis and thus we shift to more complex methodologies for trend removal. In this chapter, we employ various metrics and techniques including EOF decomposition, running climatologies, along with linear and nonlinear detrending methods to elucidate how and where natural variability changes over time. We also examine the changes to natural variability over different regions by running our analysis by basin, hemisphere, along the tropics, and different latitude belts. It remains unclear if the decrease in variance is simply by chance or if the changes are statistically significant. We test the significance of the variance change among scenarios by means of hypothesis testing.

\subsection{Methods}

In this chapter we continue to incorporate all four of the $\mathrm{RCP}$ future warming simulations in our analysis to better understand how characteristics of variability will change globally under different warming scenarios. To estimate changes to natural 
variability throughout the modeled climate, we utilize linear and various nonlinear detrending methods for our analysis. The various detrending methods include removing a least-squares linear and nonlinear quadratic trend, along with nonlinearly detrending by performing a traditional empirical orthogonal function (EOF) analysis on the multi-model ensemble comprised of 19 CMIP5 models with all six experiments available. We focus solely on analyzing sea surface temperature for this chapter.

EOF analysis has become one of the most commonly used statistical tools in climate science for identifying the spatial patterns of variability and their variation over time, and gives a measure of the importance of each pattern (Björnsson and Venegas, 1997). EOF analysis is similar to principal component analysis and is beneficial in climate science as it allows for a reduction in the number of variables within the original data without compromising the explained variance of the data. There are various ways to perform an EOF analysis (von Storch and Zwiers, 2002; Hannachi, 2004; Wilks et al., 2006; Hannachi et al., 2007) but for the purposes of this study we looked at annually averaged global SST anomalies. We begin by formatting a gridded data set of SST:

$$
X(t, s)=\sum_{k=1}^{M} C_{k}(t) u_{k}(s)
$$

Where $X(t, s)$ represents a space-time field of SST $(X)$ at time $t$ and the spatial position as $s$. For $i=1, \ldots, n$ and $j=1, \ldots, p$, the data can then be represented by a data matrix where at discrete time $t_{i}$ and grid point $s j$, SST is denoted as $x_{i j}$ and the data matrix of SST can be represented by: 


$$
X=\left(x_{1}, x_{2} \ldots, x_{n}\right)^{T}=\left(\begin{array}{ccc}
x_{11} & \cdots & x_{1 p} \\
x_{21} & \cdots & x_{2 p} \\
\vdots & \vdots & \vdots \\
x_{n 1} & \cdots & x_{n p}
\end{array}\right)
$$

The map is represented as $\left(x_{1}, x_{2} \ldots, x_{n}\right)^{T}$ where each row indicates the SST at position $x_{j}$ at all times and each column indicates the SST at time $t_{i}$ for all positions. At the $i$ 'th grid point, we represent the time average of the field as $\bar{x}_{i}$, which is calculated by:

$$
\bar{x}_{i}=\frac{1}{n} \sum_{k}^{n} x_{k i}
$$

From here, the climatology of the field is expressed as

$$
\overline{\mathbf{x}}=\left(\bar{x}_{1}, \bar{x}_{2} \ldots, \bar{x}_{p}\right)
$$

To describe variability, we must calculate the SSTs from the climatology. The departure in the SST from the climatology results in an anomaly field, which is defined by $t$ and $s$ as:

$$
x_{t s}^{\prime}=x_{t s}-\overline{\mathbf{x}}_{s}
$$

The anomaly field can also be expressed in matrix form as:

$$
X^{\prime}=X-\mathbf{1}_{n} \overline{\mathbf{x}}
$$

Where $\mathbf{1}_{n}=(1,1, \ldots, 1)^{T}$ is a vector column of ones with length $n$. Once the anomalies are calculated, we weigh the data by the cosine of its latitude. The weighting is calculated at each grid point to avoid any misinterpretations that may result from the non-uniformity of the data being distributed over the Earth's surface. We assign $\theta_{k}$ as the latitude of the k'th grid point, $k=1,2, \ldots p$, and $D_{\theta}$ as the diagonal matrix: 


$$
D_{\theta}=\operatorname{Diag}\left[\cos \theta_{1}, \cos \theta_{2}, \ldots \cos \theta_{p}\right]
$$

Next we calculate the weighted anomaly matrix:

$$
X^{\prime}{ }_{w}=D_{\theta} X^{\prime}
$$

Once the data is weighted, we can formulate and compute the EOFs by first defining a simple covariance matrix of the anomaly field:

$$
C=\frac{1}{n} X^{\prime}{ }^{T}{ }^{\prime}{ }_{w}
$$

The covariance matrix can then be decomposed via eigenalysis by:

$$
\begin{gathered}
C e_{i}=\lambda_{i} e_{i} \\
C \boldsymbol{E}=\boldsymbol{E} \Lambda
\end{gathered}
$$

Where $\boldsymbol{E}$ is a matrix with eigenvectors $e_{i}$ as its columns and $\Lambda$ is similar to an identity matrix comprised of eigenvalues $\lambda_{i}$ however, along its diagonal and surrounded by zeros. The diagonalisble nature of the transformed covariance matrix results in linearly uncorrelated combinations known as EOFs, or spatial patterns. After the eigenvalues and corresponding eigenvectors are sorted in decreasing order, the $k^{\prime}$ th EOF is simply the $k^{\prime}$ th eigenvector $e_{k}$ of $C$ and $\lambda_{k}$ is the eigenvalue corresponding to the $k^{\prime}$ th EOF which gives a measure of the explained variance by $e_{k}$ where $k=1,2 \ldots p$ or the number of spatial patterns. The measure of explained variance is usually expressed as a percentage:

$$
\frac{100 \lambda_{k}}{\sum_{k=1}^{p} \lambda_{k}} \%
$$


The projection of the anomaly field onto the $k^{\prime}$ th EOF results in the $k^{\prime}$ th principal component (PC):

$$
c_{k}(t)=\sum_{s=1}^{n} x^{\prime}(t, s) e_{k}(s)
$$

The PCs are variations in time, which are orthogonal in time with no concurrent temporal correlations between any two principal components and EOFs, the spatial pattern of variability, are orthogonal in space with no spatial correlations between any two EOFs. The original data can then be reconstructed from the PCs and EOFs as a function of time and space:

$$
X^{\prime}(t, s)=\sum_{k=1}^{M} c_{k}(t) e_{k}(s)
$$

From equation 4.14 we see the reconstruction for the full SST anomaly (SSTA) fields, which include all possible EOFs, would explain $100 \%$ of the variance found in the data and result in recreating the entire SSTA field. Several researchers have utilized the fact that for fields like SST, nearly all of the variance of the full field can be explained by using only the first few leading EOF modes (Smith et al., 1996; Mantua et al., 1997; Meyers et al., 1999; Saji et al., 1999; Yeh and Kirtman, 2004; Barbosa and Andersen, 2009; Deser et al., 2009). For the purposes of the analysis and this chapter we need to remove the warming trend in the SSTA field, which is often found in either the first or second EOF modes, along with describing the largest amount of variance and retaining all other modes of variability. Therefore, our analysis involves reconstruction of global SSTA utilizing all EOFs with the exception of the mode containing the trend. Note that for the observed data and model simulations, the EOF containing the trend may not be 
consistent across all models and is not typically described by a strictly linear principal component. The advantage of EOF analysis is because of its orthogonality constraint, allowing the data to be represented in terms of spatial modes that are uncorrelated and where EOF modes and their PCs are not linearly dependent. Most of the large-scale variability is found in lower order EOFs resulting in high amplitude spatially coherent structures. Disadvantages of the analysis also lie within the orthogonality constraint. The constraint given by the orthogonality can lead to the possible difficulty in the physical interpretation of modes associated with the complexity of the individual spatial structures. Also, if the degrees of freedom are too small in the time series or if the eigenvalues are similar, spatial features can be mixed between EOFs resulting in an overlap of natural variability modes. Another disadvantage of detrending by an EOF decomposition method is, the identification of the trend EOF may not be consistent across models resulting in a trend signal that may be split between multiple PCs. Barbosa and Anderson, 2009 attempt to isolate a long-term trend in SST and eliminate the possibility of trends over multiple PCs using a trend-EOF method from Hannachi, 2007 that retains the relevant spatial aspect of EOF techniques but considers a nonlinear ranking modification to traditional EOF analysis. While the trend-EOF technique is useful for analyzing low frequency over maximum variance patterns, it is not particularly useful on datasets longer than the satellite SST data era.

As stated above, our analysis here involves decomposing SSTA and removing the EOF that contains the largest trend, allowing us to isolate the signal of natural variability within the data in the form of a nonlinearly detrending method. We have regridded the 
data to a $1^{\circ} \mathrm{X} 1^{\circ}$ degree grid. Figure 4.1 is an example of the first $20 \mathrm{PCs}$ of the Historical experiment for the GISS-E2-H model. The example in Figure 4.1 shows a scenario where the first mode of variability contains the largest trend. For the individual Historical and RCP model members overall, the largest trend is commonly found in the leading mode. The exception to this tendency is the PiControl experiment, where the models have persistent (time dependent) radiative forcing and the models have been spun over centuries to a point where model drift is minimal. When trends are found in the leading mode of the PiControl simulation this may be a result of systematic problems in the model or issues on how the particular model was spun up. For this reason, we ran the EOF analysis over the last 100 years of each model's PiControl run. For the transient forcing simulations (Historical-RCP8.5) we reconstructed the global SSTA fields using all EOFs with the exception of the one containing the trend. Fundamentally we are able to examine how variability changes and identify which modes of variability are most affected by the anthropogenic forcing. Figure 4.2 is an example of what happens to the overall variance before and after removing the leading EOF mode from the ERSSTv4 dataset. The left panel shows the three leading EOFs of ERSSTv4 with their associated PCs of annual mean SST over the full record while the right panel displays the three leading EOFs of ERSSTv4 after the removal of the first three EOFs and their associated PCs. We see before the trend removal the leading mode displaying a global trend pattern throughout much of the global oceans and explaining $41 \%$ of the variance. The second EOF explains $16 \%$ of the variance and exhibits Pacific variability predominantly in the form of an ENSO-like spatial pattern denoted by the board triangular region along the equatorial Pacific. A weak Atlantic Ocean component is also noted in the second EOF 
pattern. The PC for the second EOF indicates the pattern varies inter-annually. The third EOF (explaining $4.2 \%$ of the variance) has strong spatial variance in the North Atlantic indicative of the AMO along with strong spatial variance in the North Pacific. Weaker, opposite signed variance is seen along the southern portion for these two oceans along with the southern Indian Ocean. The associated PC for these patterns of variability is notably decadal with considerable interannual variations. After removing the leading mode, the second EOF mode in the left panel now becomes the leading mode (right panel) explaining $27 \%$ of the variance. After removing the trend we plotted the PCs associated with these modes above the PCs before removing the trend and we see these are identical. With the residual variance after the removal of the trend being redistributed, we can compare how the spatial correlation and variance change between realizations.

We also analyze climate variability in the global oceans by removing a leastsquares linear trend to compare with the results from Chapter 3 using surface air temperature, a least-squares quadratic trend, and a running 30-year climatology from the global annual SSTs. Removing a running average helps smooth the time-series by isolating decadal modes and removing noise from smaller temporal scaled variability such as ENSO. Also, by removing the running mean the characteristics of anthropogenic forcings are untouched. The use of various nonlinear detrending methods again demonstrates the difficulty in determining the most efficient detrending method most appropriate for analyzing the GMSST. Figure 4.3 is an example of the various detrending methods mentioned above, on the ERSSTv4 global mean sea surface temperature anomaly (black line). The linearly detrended ERSSTv4 (blue line) shifts the observed 
record and displays periods of decadal warming and cooling. The removal of a leastsquares quadratic trend (orange line) and the removal of a 30-year running climatology (purple line) produce time series' that are comparable to one another and display more interannual variability throughout the record. The removal of the EOF containing the trend (yellow line) as a form of nonlinearly detrending removes any decadal variations within the time series.

\subsubsection{Hypothesis Testing: F-test}

To determine the significance of the variances changes found in this chapter, we performed an F-test on the six CMIP5 forcing experiments for the 19 individual ensemble members. An F-test is a form of hypothesis testing used to identify if two population variances are equal.

$$
\mathrm{H}_{0}: \sigma_{x}^{2}=\sigma_{y}^{2}
$$

Equation 4.15 is known as the null hypothesis where we assume two sample variances are equal. In this chapter, the F-test is applied to identify if the global distribution of variance between scenarios originates from two different sources. This hypothesis is known to follow an F-distribution. The F-distribution is similar to the student T-distribution with probability determined by known degrees of freedom. The Ftest differs in asymmetry and in characterizing the degrees of freedom by a fraction. The F-test is useful determining if a group of variables are jointly significant while a student T-test only determines if one variable is statistically significant. Here we calculated the 
variance difference for each of the individual ensemble members for the four RCP scenarios with respect to each respective ensemble members' Historical experiment. The variance difference and F-Test are calculated after removing a 30-year running climatology for each ensemble member.

The next step is to test the significance of the variance change. To determine all possible statistical values, the ratio of the two sample variances is calculated to determine the F-statistic.

$$
\mathrm{F}=\frac{S_{X}^{2}}{S_{Y}^{2}}
$$

Where $S_{X}^{2}$ in our study is the sample variance from one ensemble member's RCP scenario and $S_{Y}^{2}$ is the sample variance from the same ensemble member's Historical experiment. Here we calculated the F-statistic for each RCP scenario. The F-statistic is also known as the p-value; which is the probability the two sample variances calculated could happen by chance. Once the p-value has been calculated, the test is performed at a $90 \%$ significance level in conjunction with critical values for the specified significance level, which is used to accept or reject a null hypothesis. If the p-value is less than the significance level then the results are significant and we reject the null hypothesis. The reverse is true if the p-value is greater than the significance level then the results are not significant and therefore we cannot reject the null hypothesis.

\subsection{Removal of a Liner Trend}

To begin, we wanted to see how and where variability is going to change. We first plotted the multi-model ensemble annual global mean sea surface temperature (GMSST) 
as a box plot (Figure 4.4). For the multi-model ensemble we have 1900 simulated years after concatenating the last 100 years for each PiControl run, 2755 simulated years for the Historical experiment with each individual member ran between 1861-2005, and 1805 years for each of the RCP scenarios with each model simulated between 2006-2100. Here, the box encompasses the interquartile range (IQR), explaining $50 \%$ of the data while the median for each boxplot is denoted as a line in the box. The whiskers represent the extreme highest and lowest values found within the data. The extremes in the boxplots presented in this chapter include any outliers in the data or values greater then 1.5 X IQR. The PiControl experiment has the overall smallest distribution in temperature compared to the other five experiments with amplified forcing. The small distribution for the PiControl experiment is likely a result of the $\mathrm{CO}_{2}$ forcing levels at $280 \mathrm{ppm}$ observed during 1850 and kept steady throughout the entire run. From the PiControl to the Historical experiment, where anthropogenic forcing from GHGs are introduced, the distribution in temperature anomaly increases in both the IQR and the extremes. The median for the Historical experiment is comparable to the median of the PiControl run at $-0.0014{ }^{\circ} \mathrm{C}$ and $-0.079{ }^{\circ} \mathrm{C}$ respectively. Thereafter, from the Historical to the four RCP experiments, the overall boxplot distribution and medians for each future experiment increases with increasing amplified forcing. The increase in IQR and extremes is evidence the trend is overwhelming the signal by increasing the radiative forcing though experiments. For this reason, we want to remove the trend so we can better determine the changes to natural variability in the future as the globe continues warming.

We first remove a simple least-squares linear trend similar to the analysis in Chapter 3, from each individual model and concatenate to create the multi-model 
ensemble to see how the variability changes within the GMSST (Figure 4.5). Looking at the boxplot, all of the medians, denoted by the line within the IQR, stay closely situated near zero. The largest median is exhibited by RCP2.6 at $0.0316^{\circ} \mathrm{C}$. Again the PiControl displays a smaller overall distribution compared to the experiments with amplified forcing. From the PiControl to the Historical simulation there is an increase in the IQR by 38.7\%. Among the five CMIP5 experiments that experience increased anthropogenic forcing, the IQRs increase from the Historical simulation by $18.8 \%, 2.41 \%$, and $18.0 \%$ to RCP2.6, RCP4.5, and RCP8.5 respectively and decrease by $19.4 \%$ to RCP6.0. All of the distributions display a near normal distribution with a kurtosis close to 3 . The extremes display the largest amount of variability with an overall decrease from the Historical to the four RCP scenarios along with the Historical and RCP6.0 revealing a slight negatively skewed distribution. The results in Figure 4.5 are analogous to the results from the previous chapter, which saw an overall decrease in variability (within the extremes) as the transient forcing as a result of increased GHGs with the exception of RCP8.5, which displayed an unidentified increase in variability from RCP4.5 and RCP6.0.

\subsection{Removal of Nonlinear Trend}

Next, we nonlinearly detrend the individual ensemble members by removing a least-squares quadratic trend from the data. Removing a quadratic trend is similar to removing a linear trend with the exception that the data is assumed to follow an exponential curve. Figure 4.6 shows the boxplot for the multi-model ensemble GMSST for all six experiments after a quadratic nonlinear trend is removed from the individual members before concatenating the multi-model ensemble. Overall the medians for all six 
scenarios are once again centered near zero. From the PiControl to Historical experiment there is a $49.9 \%$ increase in the IQRs along with an increase denoted within the extremes. However, from the Historical to the four warmer RCP scenarios a reduction is found in the IQR ranging from $20.3 \%$ to $29.3 \%$. Among the four RCP scenarios, there is an expansion in the IQR and extremes from RCP4.5 and RCP6.0 and a reduction from RCP2.6 to RCP8.5 found in the whiskers that is comparable to the distributions after removing a linear trend. With similar distributions found for the linearly and nonlinearly detrended GMSST, we resampled 15-year trends over the nonlinearly detrended GMSST using the Monte Carlo resampling method in Chapter 3 to see if the distribution and results from Chapter 3 are analogous when a nonlinear trend to the data.

Figure 4.7 is the boxplot of the distribution of sampled 15-year trends over the nonlinearly detrended data. The results are similar to removing a least-squares linear trend with a all of the medians for each of the six experiments near zero; confirming as the anthropogenic forcing increases, quiescent periods of warming lasting over a 15-year period is still expected to occur. Among the distributions there is a large increase in the IQR by $60.4 \%$ from the PiControl to the Historical experiment and an overall drop from the Historical experiment to the four RCP scenarios with a gradual decrease in the IQR from $\mathrm{RCP} 2.6, \mathrm{RCP} 4.5$, and $\mathrm{RCP} 6.0$ by $31.5 \%, 38.2 \%$, and $43.8 \%$ respectively. From the Historical to RCP8.5 an overall decrease by $31.8 \%$ is exhibited with an increase of $21.9 \%$ from RCP6.0 to RCP8.5. The decrease in variability from Historical to the four RCP scenarios is statistically significant at a $95 \%$ confidence interval from an F-test on the GMSST. The result in Figure 4.7 verifies our findings in Chapter 3 where even under the assumption the GMSST experiences a deterministic trend with nonlinearity, it was found 
that overall, the variability of 15 -year trends is expected to decrease with increases in the amplified forcing from GHGs.

\subsection{Removal of Trend by EOF Reconstruction}

Following the methods above via EOF reconstruction, we next decompose annual SSTA's. Figure 4.8 shows the annually averaged multi-model ensemble of GMSST for each of the six CMIP5 experiments alongside each of the experiments respective averaged multi-model ensemble GMSST after removing the individual ensemble members' mode containing the largest trend by EOF reconstruction. All experiments with amplified forcing contained the largest trend in the leading mode for all 19 individual ensemble members. Several studies have used a similar approach to remove the leading EOF mode (Cane et al., 1997; Enfield and Nunez, 1999; Lawrence et al., 2004) however their focus was primarily on the influence of ENSO globally and how to differentiate between the low frequency variability related to ENSO and the long-term trend. Here we are only concerned with removing the leading mode as a form of nonlinearly detrending the time series. Looking at the boxplots, PiControl and Historical simulations have a median centered close to zero before removing the leading EOF. With the trend still included, all future RCP scenarios display higher medians in conjunction to their forcing constraints along with the warmer temperatures experienced in these scenarios; also the same results as in Figure 4.4. Once the EOF containing the trend is removed, variability

decreases within the Historical experiment and all four RCP experiments. The overall decrease in variability noted within the distributions after detrending suggests CMIP5 models have variability and forcing coupled together. It is commonly thought the models 
keep the two entities separate. The large drop in variability after removing the leading mode may also be a result to the overall design of EOF reconstruction. EOF reconstruction is not commonly performed without the leading mode and the restructuring of SSTA after removing the largest amount of variability may not be representative of natural variability.

Figure 4.9 shows the boxplots of only the reconstructed multi-model ensemble annual GMSST after the EOF containing the trend has been removed for individual members. Comparing only the distributions that have been detrended allows us to better identify the changes in variability between experiments once the amplified forcing is removed. Compared to Figure 4.4 when only looking at boxplots with the leading EOF removed, a long tail (leptokurtic) distribution emerges. Heavy or long tail distributions represent stochasticity, which is common for the climate system. The heavy tail distributions confirm our confidence in the models' simulation of the climate. As radiative forcing increases from the PiControl to the Historical, variability increases within the IQR by $28.7 \%$ along with increases found in the extremes. From the Historical to RCP2.6 the variability within the IQR increases by $11.1 \%$ while there is a decrease in the extremes. The larger IQR may be associated with the forcing becoming steady again in the latter half of the run; indicating an increase in natural variability by the end of the $22^{\text {nd }}$ century for the RCP2.6 scenario. For the remaining three RCP scenarios, there is a large drop in IQR from the Historical to RCP4.5, RCP6.0, and RCP8.5 by $57.0 \%, 62.1 \%$, and $49.8 \%$ respectively. RCP4.5 displays the largest positively skewed distribution at 3.34 and largest leptokurtic distribution at 20.8. Moreover, all CMIP5 experiments that experience increased radiative forcing display a leptokurtic distribution with the 
exception of RCP8.5, which exhibits a near normal distribution. For RCP6.0 and RCP8.5 the variability within the extremes is significantly smaller than the other CMIP5 experiments. Looking more closely at only those two scenarios, RCP8.5 increases in variability by $32.4 \%$ in the IQR as well as within the extremes from RCP6.0. Note that RCP8.5 displays the largest negatively skewed distribution at -1.06 . The increase in variability from RCP6.0 to RCP8.5 is consistent with the other detrending methods thus far in our analysis. It is becoming increasing likely there is something occurring within the simulated climate as a result of the vast increase in radiative forcing by the end of the next century imposed in RCP8.5 that may be increasing the overall variability within the climate system. Understanding and identifying the increase in variability will be the focus for the rest of this analysis.

\subsubsection{Modal Changes to Natural Variability}

After analyzing the changes to natural variability within the GMSST, next we want to understand and identify changes to the first few modes of variability by utilizing EOF decomposition. To identify any changes in the variance we plotted a Taylor diagram. Taylor diagrams provide a way of graphically summarizing how closely simulated patterns match observations (Taylor, 2001). All variables must be on the same grid, therefore the observed ERSSTv4 data and all CMIP5 models were regridded to a $1^{\circ}$ $\mathrm{X} 1^{\circ}$ grid. Figure 4.10 is a comparison to the first two EOFs for each ensemble member to the leading EOF pattern of ERSSTv4 before removing the trend. Each number on the diagram corresponds to an ensemble member and the colors denote the CMIP5 experiment each model member is under. The radial distance from the origin to each 
number represents the ratio of standard deviation from the CMIP5 experiment to the observed ERSSTv4. The standard deviation is calculated using the explained variance for the respective EOF modes being compared. The azimuthal positions give the weighted centered pattern correlation for the CMIP5 models' first two EOF modes compared to the first two EOF modes from the observed ERSSTv4. For the leading EOF, most model members are fairly well correlated with the observed leading mode as most correlation coefficients are found to be near 0.6. The variance explained by the first EOF in ERSSTv4 is $31.5 \%$. However, as the amplified forcing increases by experiment, the amount of the variance explained increases. The PiControl underestimates the amount of variance explained while the Historical and most model members under the RCP2.6 scenario do a better job by comparison. For the second EOF, the correlation for the PiControl drops below 0.1 with the exception of six individual model members. For higher forced experiments, RCP4.5, RCP6.0 and RCP8.5, the correlation coefficient increases closely to 0.7 on average. The variance explained by the second EOF in ERSSTv4 is $14.3 \%$. Overall, the variance explained decreases with increases in the amplified forcing by experiment.

While the variance explained decreases in the second EOF with increased radiative forcing, this does not necessarily indicate the changes and amplitude of the total variance within a particular mode as the radiative forcing increases. The percent only conveys how much of that variance is explained in a particular mode compared to the amount of variance found in other modes. Figure 4.11 is again a comparison of the first two EOF modes for each ensemble member to the first two EOFs of ERSSTv4. Each number on the diagram corresponds to an ensemble member and the colors denote the 
CMIP5 experiment each model member is under. The azimuthal positions give the weighted centered pattern correlation for the CMIP5 first two EOF modes compared to the observed ERSSTv4's first two EOF modes. However, the radial distance from the origin to each number is now represented by the ratio of total variance in each EOF mode calculated using the global variance found within the respective EOF modes by the CMIP5 experiments to the observed ERSSTv4; not the amount of variance explained by each pattern. For the leading EOF, most model members are poorly correlated with the observed leading mode as most correlation coefficients are found to be below 0.6 with the exception of RCP8.5, which displays an average 0.6 correlation coefficient across all 19 ensemble members. Overall, the Historical experiment is better correlated to the observed record compared to the other CMIP5 experiments. The higher correlation to he observed record by the Historical experiment is expected as a result of the construction of the Historical experiment, incorporating observed natural and anthropogenic greenhouse gas concentrations throughout its simulation. Interestingly, the next highest correlated scenario is RCP8.5. In terms of the variance change, as the amplified forcing increases by experiment, the variance for the leading mode appears to increase with the increases in radiative forcing. The higher radiative forcing results in more ensemble members with increased variance globally in the future by experiment. The increase in variance globally is especially noted by RCP8.5 with all ensemble members exhibiting greater variance than the observed.

For the second EOF, the correlation for the PiControl drops below 0.1 with the exception of BCC-CSM1.1-m, FIO-ESM, GFDL-ESM2M, and HadGEM2-ES. For higher forced experiments, RCP4.5, RCP6.0 and RCP8.5, the correlation coefficient 
increases closely to 0.7 on average. Overall, there is a mixture of models and CMIP5 scenarios that display a reduction and increase in variance in the second EOF pattern. For the PiControl, Historical, and RCP2.6 a reduction in the variance is displayed while for RCP4.5, RCP6.0, and RCP8.5, there is an increase in variance. The increase in variance with increased radiative forcing found in the second EOF indicates the possible increases in ENSO variability on a warmer globe as the second EOF is defined by an ENSO-like spatial pattern. We confirm the increase in ENSO variability by plotting the spatial pattern of the second EOF for the 19 ensemble members under the RCP8.5 experiment (Figure 4.12). The results of the spatial pattern in the second EOF are similar for all CMIP5 experiments that include transient forcing within their simulation (not shown). The spatial patterns within the EOFs only indicate where spatially, the variance is located.

When the global variance in the second EOF is compared to the calculated linear trend from each models leading EOF mode we see the increase in the variance associated with ENSO increase from the PiControl to RCP8.5 (Figure 4.13). One model in particular, MIROC5, displays the highest calculated variance in RCP6.0 and RCP8.5 compared to the other ensemble members. When calculating the average with MIROC5 removed (not shown) there is still a steady increase in the variance from PiControl to RCP8.5 as the trend per decade increases with radiative forcing from 0.063 to 0.352 , respectively for the multi-model average. The increase in variance with amount of GHG by experiment confirms the increasing variance to ENSO on a warmer globe as noted in Figure 4.12. 
Investigating future changes to ENSO intensity with respect to the changes in the tropical mean conditions in the next century has yielded high uncertainty associated with model dependency (Guilyardi et al., 2012; Kim and Yu, 2012; Stevenson 2012). Figure 4.14 was taken from the IPCC report (2013) and is a measure of the amplitude of El Niño calculated using the NINO3 index $\left(5^{\circ} \mathrm{S}-5^{\circ} \mathrm{N}, 150^{\circ} \mathrm{W}-90^{\circ} \mathrm{W}\right)$ across a 31 multi-model ensemble for the PiControl, a $20^{\text {th }}$ century (20C; simulation similar to the Historical), RCP4.5, and RCP8.5. Here there are little changes to the amplitude of El Niño with increased radiative forcing. However, the IPCC report asserts high confidence that ENSO will very likely remain the dominant mode of interannual variability in the coming century. An increase in ENSO variance globally, as noted here, supports the increase of global impacts imparted by ENSO. These changes in ENSO, along with the likelihood of increased moisture availability are expected to be a large influence on a regional scale. An increase in ENSO variance with radiative forcing along with increased moisture availability in the atmosphere on a warming globe may result in the intensification of ENSO-induced rainfall variability (Seager et al., 2012). Increased moisture availability is also expected to strengthen the intensity of precipitation in the global monsoon systems while the monsoon circulation is thought to weaken in the next century (Hsu et al., 2012, 2013; Kitoh et al., 2013) and in turn the mean tropical circulation (Held and Soden, 2006; Vecchi and Soden, 2007; DiNezio et al., 2009). With ENSO variance increasing shown in the previous figures, the general monsoon circulation may in fact intensify if ENSO variance increases on a warmer globe. Overall, the results shown in Figure 4.11 and Figure 4.14 suggest variance may increase on a warmer globe, however not as much as expected. The less than expected variance increase may be a result of the ENSO variance 
changing less (Figure 4.10) and may be linked to the thermodynamic constraints imposed globally on the response of the hydrologic cycle to increased anthropogenic forcing (Held and Soden, 2006) or possibly a result of the competing ocean and atmospheric dynamics responsible for changes to variability in the tropics (Vecchi et al., 2008; DiNezio et al., 2010).

\subsection{Removal of 30 year Running Climatology}

In the previous sections we showed that removing a nonlinear trend by a simple decomposition and reconstruction to 19 models under each CMIP5 scenario and by removing a linear and quadratic nonlinear trend shows (consistently across all detrending methods) a decrease in variability when the deterministic trend is removed from the amplified forcing scenarios; with the exception of RCP8.5, which displays a small increase in variability from RCP6.0 for each method. From here we decided a simpler and more straightforward way to examine changes to variability is to remove a running 30-year climatology from regridded annual SSTs from the same 19 ensemble members to isolate decadal variability. Removing a running mean suppresses high frequency noise within the signal while retaining the mean within the data. Removing a 30-year running climatology also helps determine any low frequency oscillations hidden by stochastic processes. Looking at Figure 4.15, boxplots of the ensemble GMSST display long-tailed distributions consistent with linear and nonlinear detrending methods in the previous sections. There is an increase in the IQR from the PiControl to the Historical experiment by $24.9 \%$ where anthropogenic forcing is added. The increase in the IQR is consistent with the other detrending methods previously used and may be a result of the addition of 
anthropogenic forcing in the Historical simulation or perhaps more plausibly, the addition of external forcings simulated in the Historical simulation such as volcanic eruptions. The Historical experiment also displays a slight negatively skewed distribution by -0.293 . In the recent observed record, the eruption of Mount Pinatubo in 1991 increased aerosols and gases in the atmosphere changing the amount of radiative forcing in the atmosphere resulting in a decrease in solar heating at the surface, producing global cooling in surface temperatures (Stowe et al., 1992; Robock and Mao, 1993; Minnis et al., 1993; McCormick et al., 1995; Parker et al., 1996; Soden et al., 2002). Of the RCP experiments, $\mathrm{RCP} 4.5$ experiences a more positive skewness at 0.245 towards positive anomalies. Overall, the distributions are near normal. From the Historical to the four future RCP scenarios, a drop in the IQR is observed by $17.8 \%, 20.5 \%, 22.6 \%$, and $14.6 \%$ to RCP2.6, RCP4.5, RCP6.0, and RCP8.5 respectively. An increase to RCP8.5 is once again noted from RCP4.5 and RCP6.0 and a decrease from RCP2.6 and the Historical experiment. Once more, only the increase from the PiControl and Historical, along with the decrease from the Historical to the four future warming experiments is statistically significant at a $95 \%$ significance level.

Within the variability found in the extremes, there is a drop from the Historical simulation to future experiments however, there doesn't seem to be any significant variations among the four RCPs. Even when we compare these six experiments to a 13 multi-member ensemble of a Historical experiment forced only with GHGs do we still observe a drop in variability from the Historical to Historical GHG only run (Figure 4.16). Again, from Historical GHG only to the RCP experiments there is minimal variation among the overall distribution for these highly forced experiments. The nominal 
changes to the IQRs may also indicate a decrease in variability in the future; however, the characteristics of natural variability may stay unchanged on a warmer globe as indicated by the IPCC report.

To investigate the small difference in variability we next created a scatter plot by calculating the linear (least squares) trend per decade on the raw annual SST data for each model and compared the trend to the variance of the data after removing the 30 -year mean (Figure 4.17). Figure 4.17 displays the large spread in variance among the individual ensemble members. For each experiment the trend increases with the increase in the anthropogenic forcing by experiment. The increase in variance found as a result to increased transient forcing by scenario is similar to the results shown in Figure 4.4 To get a better estimate of the change in variance by scenario, the ensemble average was calculated for each experiment (Figure 4.17b). Again, there is an increase in variance from the PiControl (0.131) to the Historical experiment (0.146). A small decrease in variance is observed from the Historical to RCP2.6 to 0.140. However, RCP2.6 rests close to the Historical run in terms of the trend, which is expected as RCP2.6 this experiment is close to the Historical regarding the amount of radiative forcing imposed within the simulation throughout the next century. The variance continues to decrease among the four future RCP scenarios marginally until RCP8.5 where an increase in variance is denoted. The increase in variance exhibited in RCP8.5 is synonymous to the increases in variance observed with calculating the GMSST among the various detrending methods.

With the various increases and decreases that take place among the CMIP5 experiments found to occur in the box and whiskers and scatter plots, we next masked out 
the five largest ocean basins, the Atlantic, Pacific, Indian, Southern and Artic Oceans. We calculated the linear (least squares) trend per decade for the raw annual SST data for each basin and plotted against the variance of the data after removing the 30-year mean in each basin to see if we can isolate where variability changes the most. The ensemble average of the linear trend versus the variance for the five ocean basins is shown in Figure 4.18. For all ocean basins there is an increase in variance from the PiControl to the Historical run with the largest increase in variance found in the Pacific Ocean by 0.013. From the Historical run to the RCP runs a decrease in variance occurs for all basins except for the Artic Ocean. For the Artic Ocean, the variance increases steadily with amplified forcing; confirming that melting sea ice is to be expected on a warmer globe and will be expected to add variance. The addition of variance in the Artic may also be result in the variance increase noted from RCP6.0 to RCP8.5 in the GMSST discussed in previous sections and is also noted by the variance maps. The Atlantic Ocean alternates between increasing and decreasing variance between experiments from the Historical to RCP8.5. The Indian Ocean overall has the smallest amount of variance when compared to the other ocean basins and displays a marginal increase in variance from RCP2.6 to RCP8.5. The Pacific and Southern Oceans exhibit a decrease in variance from the Historical to the future experiments and continues decreasing with the increase in radiative forcing by scenario until RCP8.5 where there is an increase in variance. The increase in variance in RCP8.5 may be the result of the increases in variance noted in the boxplots from previous sections.

We looked at variance changes by ocean basin and next we look closer into where the variance may be increasing by latitude belt. We calculated the multi-model 
ensemble variance on the SSTA after removing a 30-year running climatology and linear (least squares) trend per decade on the annually averaged raw SST by latitude belts $5 \mathrm{~S}$ $5 \mathrm{~N}, 10 \mathrm{~S}-10 \mathrm{~N}, 15 \mathrm{~S}-15 \mathrm{~N}, 30 \mathrm{~S}-30 \mathrm{~N}, 45 \mathrm{~S}-45 \mathrm{~N}$, and $60 \mathrm{~S}-60 \mathrm{~N}$ for the individual ensemble members and plotted the average linear trend against the average variance for the 19 ensemble members (Figure 4.19). Globally, the linear trend increases over all latitude belts as the amplified forcing increases by scenario. The increase in the linear trend with GHGs is consistent with the results in previous sections. The scatterplot displays an increase in variance from the steadily forced run to runs with increases radiative forcing for each latitude belt. The increase in variance coinciding with increases in well-mixed GHGs is also verified in results from previous sections. The largest overall variance is found in the tropics between $5 \mathrm{~S}$ and $5 \mathrm{~N}$ latitudes. The tropics also experience the largest increase in variance by 0.35 between the PiControl and Historical and the largest decrease of 0.016 in variance from the Historical to RCP2.6. Overall, the tropics also exhibit the largest variance decrease between latitude belts as noted between latitude 5 and 10 degrees latitude. Variance along the tropics is dominated by interannual variability (Battisti and Hirst, 1989; Carton and Cao, 1996; Mantua et al., 1997; Saji et al., 1999; Ruiz-Barradas et al., 2000; Huang and Kinter, 2002). The variance overall continues to decrease into higher latitude belts until the mid-latitudes (45S and $45 \mathrm{~N}$ ) where an increase in variance is noted between latitudes 45 and 60 degrees. The increase in variance again may be a result to the addition of melted ice near the polar regions that is likely to occur on a warmer globe or possibly changes to atmospheric modes of variability that occur in higher latitudes. 
Next we look at how the SST gradient will change on a warmer globe when compared to changes in variance. In modeling studies, tropical SSTs are found to be a minimal driver in the expansion and contraction of the tropical atmospheric belt (Mitas and Clement, 2005; Johanson and Fu, 2009; Lu et al., 2009, Allen et al., 2014). The tropical atmospheric belt has been found to have-widened since the satellite era $(\mathrm{Hu}$ and Fu, 2007; Lu et al., 2007; Seidel et al., 2008). However, it is unclear on the full response to the meridional SST gradient from increased GHGs. Here we calculated the SST gradient in Kelvin and variance for each individual ensemble member between latitude belts $0-20 \mathrm{~N}, 0-45 \mathrm{~N}, 0-60 \mathrm{~N}, 0-80 \mathrm{~N}, 20 \mathrm{~N}-45 \mathrm{~N}$, and $20 \mathrm{~N}-60 \mathrm{~N}$ and averaged to plot the multi-model ensemble. Figure 4.20 is a scatter plot of the SST gradient in the northern hemisphere compared to the variance within these latitude belts for all six CMIP5 experiments. Overall, variance is seen to increase between the PiControl experiment and experiments with increased radiative forcing for all latitude belts plotted. The variance also increases with latitude. Once amplified forcing is added to each experiment, the overall change in variance and SST gradient between experiments is too similar to denote in the scatterplot. As a result, the small changes in SST gradient and variance between scenarios it is hard to identify any verifiable correlation between the changes in variance and SST gradient. An increase in the SST gradient is seen between PiControl to the Historical run for all six latitude belts. The smallest increase in variance is along the tropics between the equator and $20 \mathrm{~N}$ by 0.07 Kelvin while the largest increase in variance is found between the equator and $80 \mathrm{~N}$ by 0.16 Kelvin. In the northern hemisphere, the tropics between the equator and 20N, displays the smallest overall SST gradient for all scenarios. The tropics also exhibit an increase in the SST gradient with increased 
radiative forcing from the Historical to RCP8.5 by 0.21 Kelvin. Between the equator and $80 \mathrm{~N}$ there is a large increase in SST gradient by 1.1 Kelvin from the Historical to RCP85. The increase in variance may be a result to cooler temperatures from melting ice. SST gradients calculated between the equator and $45 \mathrm{~N}$ and $20 \mathrm{~N}$ to $45 \mathrm{~N}$ display a steady decrease from the Historical to RCP8.5. For SST gradients calculated to $60 \mathrm{~N}$ there is an overall increase from Historical to RCP8.5 with a small decrease noted at RCP6.0. The changes to SST gradient may be a result of the differences between the individual models future projected warming and their effect on melting ice.

To see how the latitudinal SST gradient change with variance change in the southern hemisphere we calculated the SST gradient for each model member's respective belts between $0-20 \mathrm{~S}, 0-45 \mathrm{~S}, 0-50 \mathrm{~S}, 0-60 \mathrm{~S}, 20 \mathrm{~S}-45 \mathrm{~S}$, and 20S-60S averaged over for the multi-model ensemble (Figure 4.21). Overall, the SST gradient increases with latitude. The largest increase in SST gradient from PiControl to RCP8.5 is between the equator and $60 \mathrm{~S}$ by 1.49 Kelvin. Generally, the SST gradient steadily increases from the PiControl run to RCP8.5 for all latitude belts except for $45 \mathrm{~S}$ where there is a drop in RCP6.0 and then an increase again to RCP8.5. Interestingly, in the northern hemisphere this latitude experiences a steady decrease in SST gradient. The increases in SST gradient in the southern hemisphere across the tropics and mid-latitudes confirms the warm get warmer and cold gets colder argument where with an increase in variance there are equal probabilities for warm and cold extremes (IPCC, 2013). 


\subsubsection{Spatial changes to variance}

To understand the unexpected increases in variance between these future scenarios, we plotted the ratio of two variances for the multi-model ensemble. We first calculated the ratio of variance for each individual model between the PiControl to

scenarios with transient forcing, i.e. $\frac{\operatorname{Var}(\text { Historical or } R C P)}{\operatorname{Var}(\text { PiControl })}$ and then the ratio between the Historical to the four RCP experiments, $\left(\frac{\operatorname{Var}(R C P)}{\operatorname{Var}(\text { Historical })}\right)$ followed by averaging over the ratios for the multi-model ensemble. It was not necessary to standardize before averaging as the variance ratios between the different scenarios were calculated for the individual models first and all models use the same physics when ran for each scenario. We chose to compare the ratios to help identify where natural variability changes by increasing the amplified forcing from a steady forced state. A ratio of 1 corresponds to no change in variance while values $>1(<1)$, result in an increase (reduction) of variance between the experiments. When calculated globally, changes to variance in the Polar Regions are exceptionally large and mask changes to variance in the tropical and mid-latitudes. The large variance in the Polar Regions is expected from our physical understanding because of the current, rapidly melting ice in these regions. The increase in variance verifies the increases in variance found in the scatter plots of the changes in variance with increased radiative forcing by basin and latitude belts.

To evaluate the changes found in the Polar Regions, we plotted the ratio of variance over the Artic (Figure 4.22) and Antarctic (4.23) between the Historical and four RCP future experiments. We see in RCP2.6 two areas of increasing variance near the Greenland and Norwegian Seas and also along the Chukchi Sea. As the radiative forcing 
increases from RCP2.6 to RCP8.5 the variance increases over $450 \%$ in the regions mentioned and expand unanimously throughout the Artic Ocean in addition to an extension into the Baffin Bay. The large increase in variance found along the Arctic may be the explanation for the large increases in variance found in Figure 4.13. Sea ice in the Arctic exhibits interannual variability tied to local surface air temperatures and wind anomalies that can be driven by large-scale atmospheric circulation patterns (Prinsenberg et al., 1997; Deser et al., 2000). With Arctic sea ice rapidly declining (Zhang and Walsh, 2006; Stroeve et al., 2007; Cosimo et al., 2008) and an ice-free Arctic Ocean projected to occur during the summer (at the very least) by the end of the next century (Stroeve et al., 2007; Wang and Overland, 2009; Overland et al., 2011), the increased amount of open water in the Arctic, along with the reduction in albedo would have significant effects on the atmospheric circulation in the high latitudes (Rahmstorf, 1999; Johannessen et al., 2004; Singarayer et al., 2006; Deser et al., 2010; Liu et al., 2012). The result of these effects may give rise to new mechanisms of variability in the atmosphere explaining the increased variance found in RCP8.5.

Along the Antarctic (Figure 4.23), the increase in variance extending into the Weddell Sea and southern Indian Ocean also increases with the addition of GHGs by each RCP experiment. Similar results were found when the variance was plotted with respect to the PiControl simulation (not shown). While the Arctic Ocean has displayed an extensive loss to sea ice since the 1970s, the Antarctic has experienced a statistically significant increase in sea extent when averaged along the continent (Zwally et al., 2002; Cavalieri and Parkinson, 2008; Cosimo and Nishio, 2008). The increase in sea ice extent has been linked to the reduction in stratospheric ozone and oceanic mechanisms in the 
Southern Ocean (Zhang 2007; Turner et al., 2009). It is unclear how the sea ice extent will change under increased greenhouse gas concentrations. However a modeling study by Smith et al., 2012 suggests the projected ozone recovery will mitigate the loss of Antarctic sea ice extent expected to occur with increased GHG concentrations in the next century.

To remove the influence of the variance changes derived by the Artic and Antarctic, we plotted the variance maps for latitudes between $60 \mathrm{~S}$ and $60 \mathrm{~N}$. Figure 4.24 shows the variance ratios calculated from the RCP experiments with respect to the Historical experiment between $60 \mathrm{~S}$ and $60 \mathrm{~N}$. Here we have plotted any value below one in blue to denote a reduction in variance and red for any value larger than one for an increase in variance. A broad area of reduced variance is found along the southern portion of the Atlantic, Indian, and Pacific Oceans. Overall, a reduction in variance is more prominent along the tropical oceans. Areas with a noted reduction of variance are also found along the western tropical Pacific for all four panels. The largest area of reduced variance is exhibited for RCP4.5 along the equatorial Atlantic, Indian oceans, and along the western Pacific while RCP2.6 displays most of the reduced variance along the off-equatorial region near the western warm pool in the Pacific. An area of increasing variance is noted along the northern Atlantic Ocean in RCP8.5 corresponding to the region where sea ice is melting near the Artic.

Figure 4.25 is an example of a point-wise F-test plotted for only one ensemble member, CCSM4. Contours plotted are the difference in variance between each RCP experiment and CCSM4's Historical experiment. Red contours indicate an increase in variance over the next century while blue contours are a decrease in variance. The 
hatched areas denote statistically significant regions with p-values calculated below the significance level of 0.10 . Once the F-test was calculated for each individual model member we separated the variance change from Historical to each RCP by variance decreasing in the RCP scenarios and variance increasing. Separating the variance by increasing vs decreasing areas, allows us to better compare statistically significant variance change globally.

Figure 4.26 is a robustness plot showing the percent of ensemble of members displaying a statistically significant increase in variance over the next century. The variance change was calculated for each RCP scenario from the Historical experiment. Here the variance is not normalized as the variance change and statistical significance tests were calculated for the individual ensemble members before averaging. The red color indicates areas of expected increases in variance and the heavier shading indicates a higher number of ensemble members with a statically significant increase in variance at a $90 \%$ significance level. For all four RCP scenarios over $80 \%$ of the ensemble members agree on increasing variance in the Artic Ocean. The agreement among ensemble members was also noted in the variance ratio maps. Good agreement for a variance increase also occurs along the Antarctic region among the ensemble members. In the northern hemisphere all four RCP scenarios also show agreement among ensemble members for an increase in variance along the North Atlantic. The North Atlantic region is also associated with the location of the Atlantic Meridional Overturning Circulation (AMOC) and extending out across the extra-tropical Atlantic. The extension across the extra-tropical Atlantic is spatially similar to the Atlantic Meridional Mode (Servain et al., 1999; Xie and Carton, 2004; Chiang and Vimont, 2004). There is fair agreement among 
the ensemble members for a variance increase in the Sea of Okhotsk. Other areas of variance increases found in all four RCP scenarios are along the eastern tropical Pacific synonymous to the signature of ENSO and the tropical eastern portion of the Indian Ocean. Another signature of increasing variability along the northern Pacific looks similar to the Pacific Meridional Mode (Chiang and Vimont, 2004). In the southern Pacific, RCP2.6 displays the largest increase in variance that extends from the western tropical Pacific. An increase in variance is also seen for all four RCPs in the southern Indian Ocean for all four RCP scenarios. A smaller percentage of ensemble members agree on several areas of increasing variance along the tropical Indian and Pacific oceans with RCP4.5 displaying the largest increases in variance in these regions. Interestingly, the statistically significant variance increase in RCP4.5 has a similar spatial distribution to ENSO. In RCP4.5, an increase in variance along the equatorial Pacific is noted where the Central Pacific El Niño is located. However, the increase in variance is consistent through all scenarios. RCP4.5 also displays the largest increase throughout the tropical Indian Ocean synonymous with Indian Ocean warming throughout the basin. The increases in variance along the eastern portion of the Indian and Pacific oceans could be associated with changes to Bjerkenes feedbacks or may be a result of changes to ENSO modulation in the Indian Ocean noted in the increase in variance over the Central Pacific. Chu et al., 2014 found under RCP4.5 radiative forcing, enhanced air-sea coupling over the Indo-Pacific region, in response to El Niño activity, may result in favorable conditions for a positive IOD phase deriving cooler temperatures over the eastern Indian, which are associated with enhanced Bjerkenes feedbacks. 
The percent of ensemble members predicting a statistically significant decrease in variance is shown in Figure 4.27. Figure 4.27 is similar to Figure 4.26 in calculation except the blue shade denotes the percent of ensemble members with a statistically significant decrease in variance globally. Again, the darker shade indicates the higher percent of ensemble members agreeing on the variance decrease. For a decrease in variance, only RCP2.6 and RCP4.5 show some agreement in a small area of variance decrease in the Artic. There is again good agreement along the Antarctic and North Atlantic Ocean for all four RCP scenarios. Modeling studies found the differences in the spread of sea ice extent along the Antarctic were linked to zonal wind speed and cloud fractions (Holland and Kwok, 2012; Mahlstein et al., 2013). Variance decreases are also noted along the tropics and sub-tropics in the Pacific, Atlantic, and Indian oceans in all four RCP scenarios. A decrease in variance is calculated to occur within a large swath in the tropical regions among the RCP4.5 and RCP8.5 scenarios. More notably, for all four RCP scenarios a decrease in variance is seen along the Northwestern Pacific, Indian, and Atlantic Oceans. The decrease in variance may be associated with the changes in the warm ocean currents along adjacent continents, such as the Gulf Stream and Kurishio current. Also, the location of the western warm pool region in the Pacific is where a large area of convection lies and is strongly coupled with ENSO oscillations. The decrease in variance in the western warm pool may be a result of the complex cloud feedbacks (Ramanathan and Collins, 1991; DiNezio et al., 2009). Along the Indian Ocean, the change in variance is comparable to the spatial characteristics of the IOD as well the observed increase in warming throughout the basin. 
Looking at Figures 4.26 and Figure 4.27 the percent of ensemble members showing increases and decreases in variance are similar in terms of spatial structure. The only question now is what will the sign of the variance change actually be in these regions on a warmer globe? Tebaldi et al., 2011 argue the sign of the change may not be relevant if observationally the change is related to internal variability. However, they also state the model agreement is only meaningful if the change is statistically significant; which in our case is, as we are testing for statistical significance. Moreover, their study does not take into account the mechanisms within the individual models that are responsible for their future behaviors. Therefore, we wanted to take a closer look at what is happening to the variance change among the individual ensemble members to see if we can find any differences in the sign of variance change among ensemble members from the same modeling agency. Figure 4.28 shows a difference in the variance change between the National Center for Atmospheric Research's (NCAR) CCSM4 and CESM1CAM5 models and Figure 4.29 shows the difference in variance change between the Geophysical Fluid Dynamics Laboratory's (GFDL) physical GFDL-CM3 model and their two Earth system models, GFDL-ESM2G and GFDL-ESM2M respectively. Interestingly, for both modeling agencies the canonical physical coupled climate models results in the opposite sign regarding the variance change compared to each modeling agencies respective earth systems model (ESM). General Climate Models (GCM) simulate the physical climate using four separate components simultaneously, an atmospheric, ocean, land, and ice model. These four components are then ran through a coupler that exchanges the fluxes between each component and current state information as the model progresses in time. ESM's include the same major components used in 
GCMs but are more advanced by incorporating biogeochemistry, land use, and an interactive carbon cycle.

In Figure 4.28, we compare the variance change for all four RCP scenarios between CCSM4 and CESM1-CAM5. Both NCAR models have an increase in variance in the Artic and Antarctic region as seen in the variance ratio maps as well as the robustness plots of the statically significant variance changes. However, the largest changes in variance are seen in the tropical Pacific. For CCSM4 a decrease in variance is seen in the tropical Pacific region while CESM1-CAM5 shows an increase in variance. Both variance changes look similar to ENSO. The difference in sign can be seen for all four RCPs between these two models. For CESM1-CAM5 the increases in variance are broader in the southern hemisphere then the decreases in variance in CCSM4. Both CCSM4 and CESM1-CAM5 are essentially physical GCMs (Neale et al., 2010; Gent et al., 2011; Meehl et al., 2012) but they differ in their forcing within the atmospheric component. When looking at Table 4.1 of included forcings documented in the IPCC report, the Historical experiments for these two models are forced almost identically with the exception of aerosol forcings. CCSM4 uses prescribed concentrations of each aerosol component while CESM1-CAM5 uses concentrations of forcing agents that are calculated interactively from the prescribed emission as the model marches forward in time. Another notable difference in forcing agents between these two models is that CESM1-CAM5 includes cloud albedo effect and cloud lifetime effect while CCSM4 only includes the direct effect of clouds. A special study conducted by Meehl et al., 2013 compared the two models and found that CESM1-CAM5 is more responsive to changes in external forcings. These responses result in slower heat uptake from the oceans causing 
greater warming of the surface air temperature. The inclusion of direct and indirect effect of clouds results in additional warming over the northern hemisphere oceans (Meehl et al., 2013) and may be the reason for the difference in sign between these two models.

We also compare the variance changes for GFDL's three models, CM3, ESM2G, and ESM2M (Figure 4.29). Where CM3 is their canonical physics model and ESM2G and ESM2M are their two ESM models. All three models show an increase in variance along the Artic region for all four RCP scenarios. For all three models there is a dipole of variance change found in the North Atlantic for the various RCP scenarios. Small increases in variance along the equatorial Indian Ocean are seen in GFDL-CM3 similar to the increases in variance found in the variance ratio maps. Along the Antarctic coast, GFDL-CM3 displays the largest decreases in variance in the Weddell Sea for all RCP experiments. The largest difference in the variance change among the three models exists along the Pacific. In the Pacific region, CM3 exhibits an overall increase in variance along the tropical Pacific in RCP4.5 and extending into the southern Pacific Ocean. The ESM2M model displays a robust decrease in variance along Pacific Ocean for all four RCP experiments. The ESM2G model displays a larger decrease in variance along the North Pacific in the PDO-like horseshoe pattern for each future scenario and is followed by an increase in variance on the equatorial Pacific in RCP8.5. The differences in the changes to variance are likely because of the individual biases to climate sensitivities and feedback mechanisms simulated by the individual models. For GFDL's ESMs, the main difference between these two models is the physical ocean component (Dunne et al 2012). GFDL-ESM2G is run with an independently developed isopycnal model and GFDL-ESM2M uses a pressure-based vertical coordinate system that is used along the 
developmental path. The difference in ocean configuration results in GFDL-ESM2M with stronger ENSO variability and may be the result of the robust decrease in variance along equatorial Pacific in Figure 4.29. When comparing the forcing agents in Table 4.1 both ESMs are forced identically with GHG and aerosol forcing agents included via prescribed concentrations while forcing agents in GFDL-CM3 are calculated interactively for most GHG and aerosol components. Note any cloud forcings are only prescribed in GFDL-CM3 and not included in either GFDL-ESM2G or GFDL-ESM2M.

\subsection{Conclusions}

In this chapter, we began by looking at how natural variability will change in the future as the globe continues to warm. We began by analyzing the data through various linear and nonlinear detrending methods. We included a technique involving the use of a traditional EOF decomposition, where we reconstructed the data after removing the leading EOF mode containing the largest trend for the individual ensemble members. We also removed a 30-year running climatology to elucidate how and where natural variability changes over time. We also examined globally the changes to natural variability by running our analysis by basin, hemisphere, along the tropics, and different latitude belts. We tested the decreases and increases in variance using a point-wise F-test.

We saw that by plotting the annual multi-model ensemble GMSST the trend overwhelms the temperature signal as the amplified forcing and global surface temperature increases. Previous studies confirm that currently the signal of amplified forcing is beginning to emerge in lower latitudes masking the signal of natural variability (Mahlstein et al., 2011; Hawkins and Sutton., 2012). The first method we used to remove 
the trend from the GMSST was detrended using linear least squares regression similar to the detrending method in Chapter 3 and found the largest amount of variability within the extremes. We discovered an overall decrease within the extremes occurred between the Historical to the four RCP scenarios. These results are analogous to the results from Chapter 3, where an overall decrease in variability (within the extremes) was found to occur as the transient forcing associated with increased GHGs, with the exception of RCP8.5, which displayed an unidentified increase in variability from RCP4.5 and RCP6.0.

Taking a look at the shape of the four RCP scenarios after removing a linear trend would result in high variability. When comparing the shape of the GMSST is it evident that the trend is not fully linear for any of the amplified forcing scenarios; suggesting that removing a linear trend may have added noise to our boxplot distributions. Therefore, we next tried to remove a least squares quadratic trend for the individual ensemble members. When looking at the averaged multi-model ensemble GMSST we see an increase in variability from the PiControl run to the Historical experiment and a small steady reduction from the Historical to the RCPs with the exception for RCP6.0 which observes an increase in variability. With similar distributions found for the linearly and nonlinearly detrended GMSST, we resampled 15-year trends over the nonlinearly detrended GMSST using the Monte Carlo method in Chapter 3. The result of the statistical analysis on the nonlinearly detrended GMSST verifies our findings in Chapter 3 where even under the assumption the GMSST experiences a deterministic trend with nonlinearity, it was found that overall variability is expected to decrease with increases in amplified forcing from GHGs. 
Next we used a unique EOF decomposition method to nonlinearly detrend the data. We did detrended by removing the EOF mode containing the largest trend. For all experiments that were run with amplified forcing, the leading mode for each ensemble member was found to contain the trend. We then decompose each member individually and remove the leading mode. The EOF decomposition and removal of the leading EOF was followed by reconstruction of the dataset with all EOF modes remaining after removing the largest trend. We found for the multi-model ensemble GMSST there is a strong drop in variability found within the IQR in RCP6.0 and RCP8.5 when the trend is removed. We then used Taylor Diagrams to graphically compare the first two EOF modes to the observed ERSSTv4 EOF modes and saw that that the leading mode increases in variance explained as the transient anthropogenic forcing increases by experience. Followed by a decrease in the variance explained for the second EOF. The spatial pattern for the second EOF describes the variability associated with ENSO. When calculated the actual variance within a full 3 dimensional field of the second EOF we see the variance actually increases with increased radiative forcing when compared to the second EOF of the observed ERSSTv4 record. The increase in variance within the full fields suggests the variance may increase on a warmer globe, however not as much as expected as the variance associated with ENSO may be changing less. The increase in variance associated with ENSO has global impacts that include effects on the overall circulation on the global monsoon as well as increases precipitation in many areas. The increase in precipitation is also an effect of the increased moisture availability in a warmer atmosphere. 
Next we decided to simply remove a running 30-year climatology for the individual ensemble members. Removing a 30-year running climatology allows us to detrend the data by removing higher frequency noise but still retain the mean of the data. We found in the multi-modeled ensemble GMSST there again was a jump in variability between the PiControl and Historical runs and then a drop from the Historical to the four RCP experiments. However, among the future experiments there isn't much variation in the variability. The marginal changes to variability in the box and whiskers plot was similar when we compared the experiment to a multi-model ensemble of a Historical GHG only simulation. The lack of variation among the RCPs suggests overall the characteristics of variability may not change on a warmer globe. When looking at the variance by ocean basin when compared the linear trend per decade by basin, this is confirmed by a steady increase in variance in the Artic Ocean by forcing scenario and alternating variance increases and decreases in the Atlantic and Indian Oceans by forcing scenario.

In contradiction to the variance maps there is a smaller decrease in variance from the PiControl to the future RCP scenarios than from the Historical run to the RCP experiments in the Southern Ocean. Next we calculated the variance changes by latitude belts and found the largest increase in variance is in the tropics between $5 \mathrm{~S}$ and $5 \mathrm{~N}$ of the equator. Secondary increases in variance are between the equator and $80 \mathrm{~N}$ where there is expected ice melt as the surface temperatures continue to warm. The largest decrease in variance is found in the mid-latitudes at $45 \mathrm{~N}$. Changes to SST gradients result in similar increases in variance in the tropics and between the equator and $80 \mathrm{~N}$. The largest decreases in SST gradients with variance are found when calculated to $45 \mathrm{~N}$. In the 
southern hemisphere overall the SST gradient increases with latitude and only some decreases are found between CMIP5 modeling experiments.

We plotted variance maps depicting the percent ratio of variance change from both the PiControl and Historical run to the RCP experiments. The largest variance increase is found along the Artic, Antarctic, and North Atlantic Oceans with secondary smaller increases in variance along the eastern Indian and central equatorial Pacific Oceans. Increases in variance seem to be heavily influenced by the melting sea ice in the northern hemisphere; with secondary increases in variance located along the tropical region; possibly a result of increases in ENSO variability. We then tested the statistical significance of the variance changes by the individual ensemble members and found the variance changes are statistically significant. However, the individual ensemble members disagree on the sign of the variance change. Statistically significant variance increases are found in the Southern Ocean along Antarctica, the North Atlantic, the eastern tropical Pacific in RCP4.5 and the Indian Ocean. Statistically significant decreases in variance are also seen along the same regions for all four RCP scenarios with larger areas of a reduction in variance along the equatorial and western warm pool region in the Pacific.

The difference in sign may be a result in the physical mechanisms between the canonical GCMs and ESMs. It was found for NCAR's models to exhibit similar variance changes along the Pacific with a difference in sign. The difference in the sign of the variance change was also noted for GFDL's three working models however not as robust. When comparing the forcing agents added between the GCM and ESM models, differences in the changes in variance may be a result of the addition of cloud radiative effects. When cloud radiative effects are added in the CESM1-CAM5 model, the variance 
was shown to decrease in the tropical Pacific while increasing in GFDL-CM3. Within ESM's the changes in variance may be a result in the oceanic dynamics taking place within the model. Pressure-based vertical coordinates result in a robust decrease in variability along the tropical Pacific.

By simply looking over the different metrics and techniques there seems to be a clear consensus among them on a steady decrease in variability as the amplified forcing increases by experiment with the exception of RCP8.5 which sees an increase in the IQR and decrease in the extremes. The agreement among methods clarifies that natural variability will continue to influence SST on a warmer globe. The changes in variance are found to be a result of melting ice in the Artic and changes to variability in the tropical oceans. 

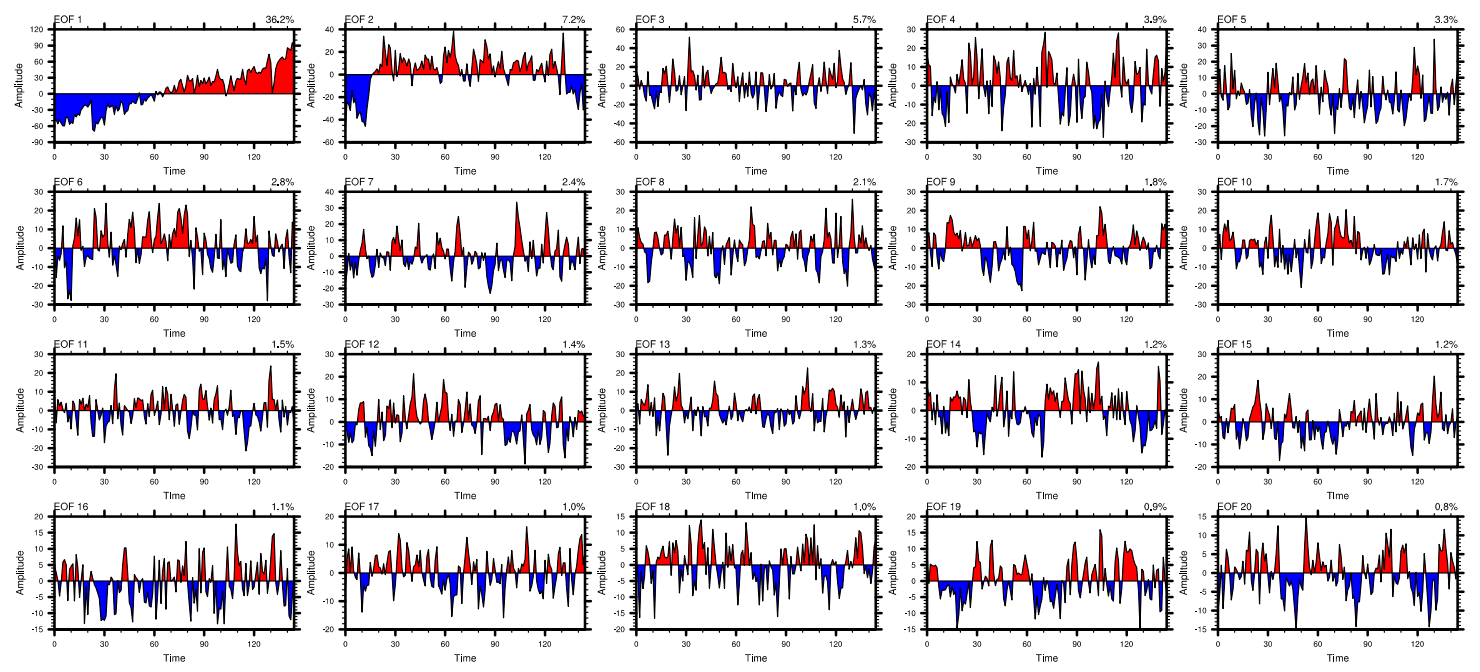

Figure 4.1:Principle Components for the first 20 EOFs in GISS-E2-H under the Historical experiment (1861-2005). The trend is observed in the leading mode (EOF 1). 

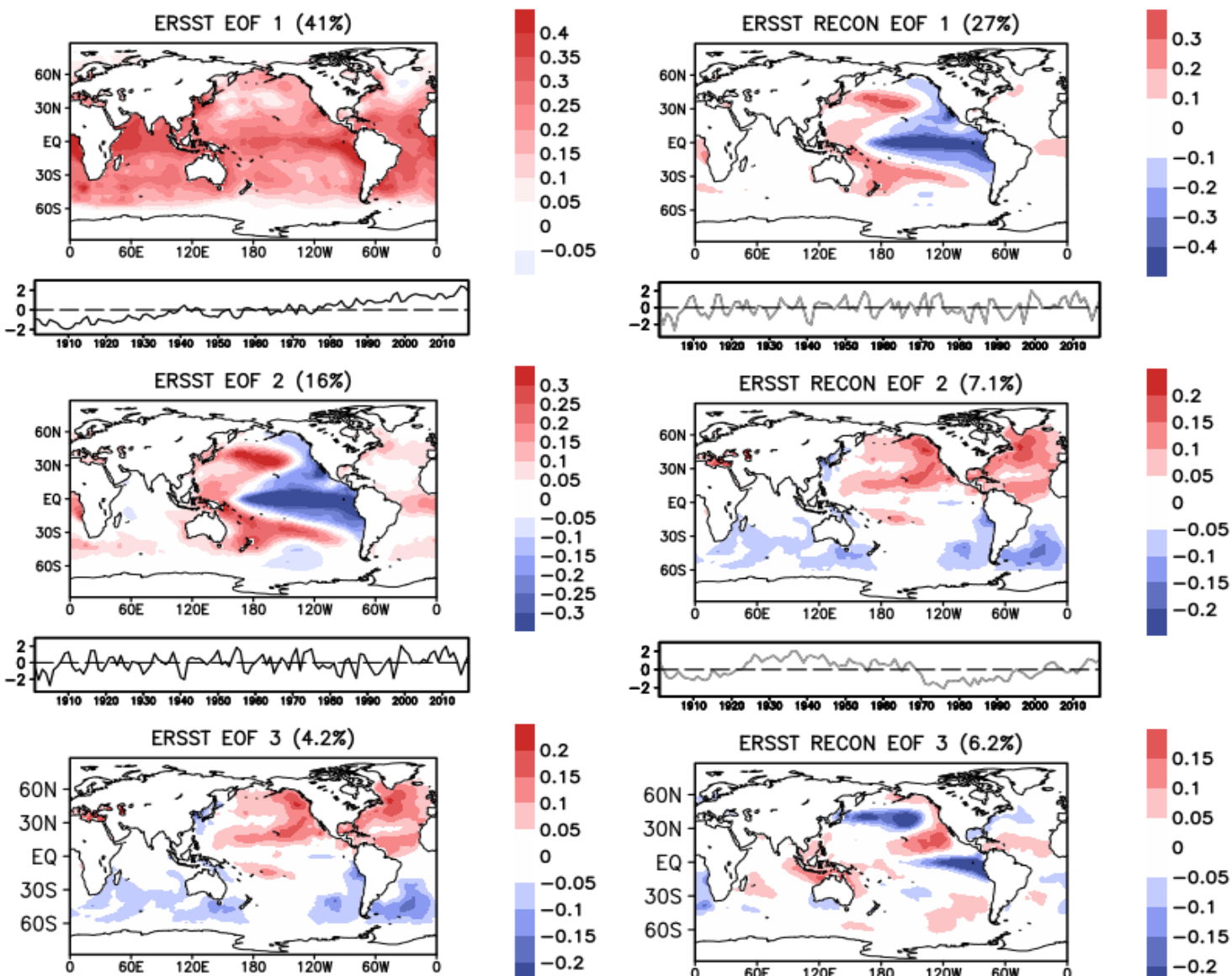

2.
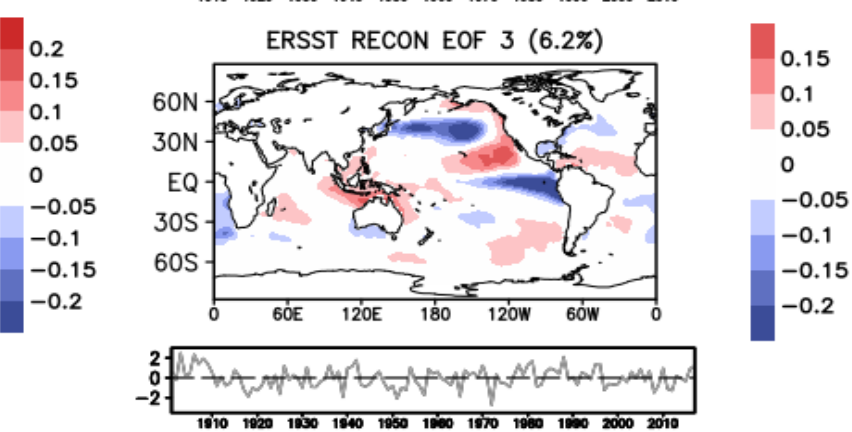

Figure 4.2: The (left) three leading EOFs with variance explained (in percent) of ERSSTv4 with their associated PCs of annual mean SST over 1901-2017 and (right) the three leading EOFs of ERSSTv4 after the removal of the first EOF and associated PCs. 


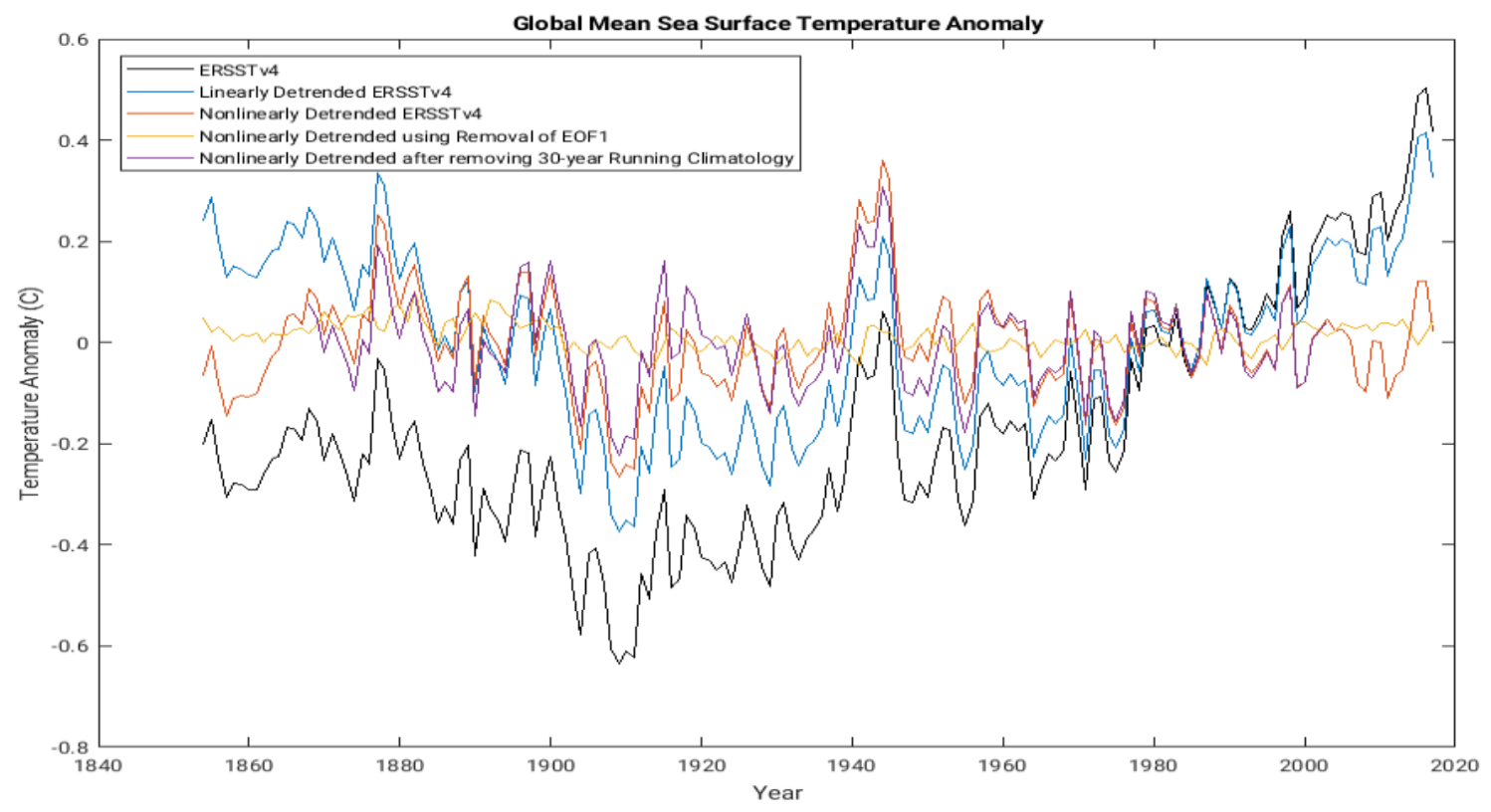

Figure 4.3: Observed annually averaged ERSSTv4 global mean sea surface temperature anomaly (black line) with the linearly detrended ERSSTv4 time series (blue line), nonlinearly detrended ERSSTv4 time series using a least-squares quadratic trend (orange line), removal of the EOF containing the largest trend (yellow line), and removing a 30year running climatology (purple line) from 1854-2017. 


\section{Annual Ensemble GMSST}

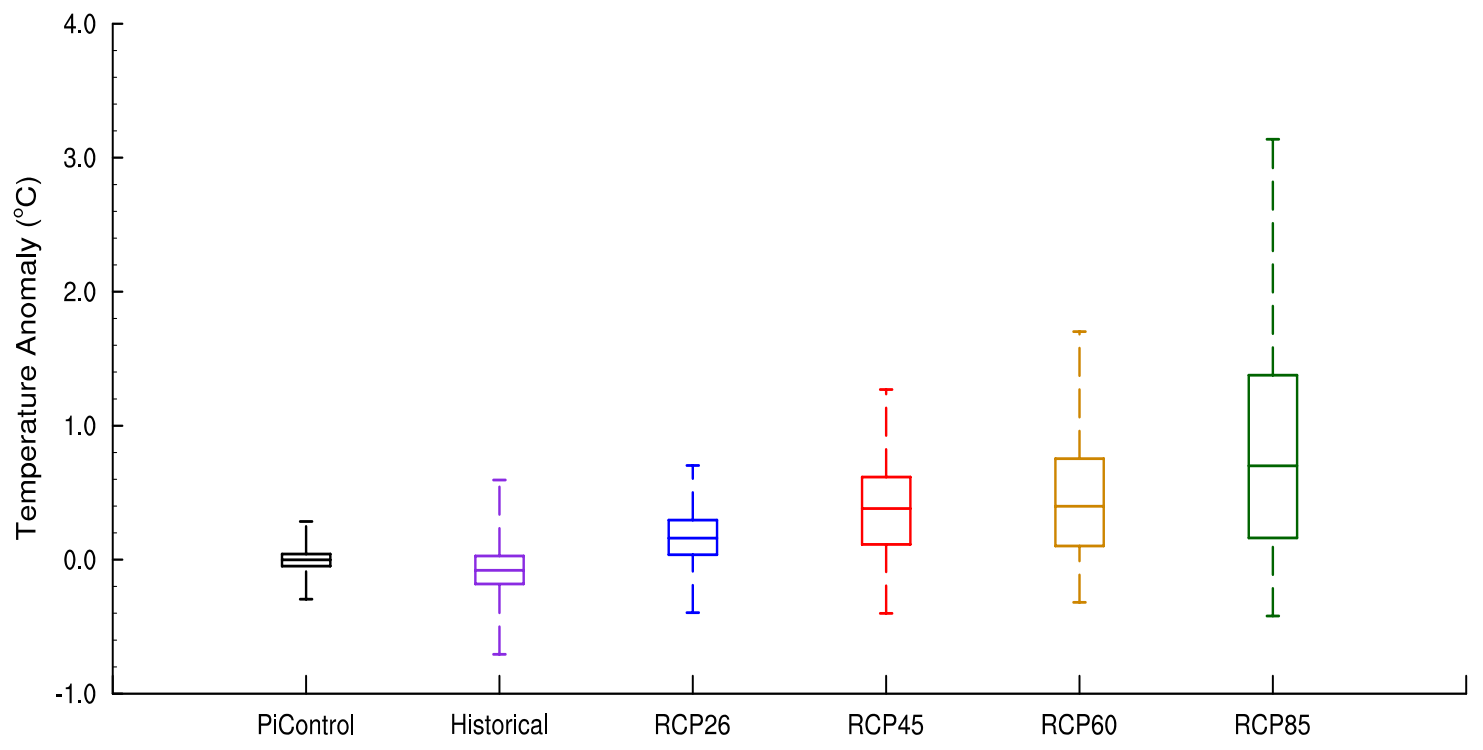

Figure 4.4: Box and whisker plots of the annually averaged multi-model ensemble GMSST before any detrending method is applied. The multi-model ensemble consists of 19 ensemble members each detrended from the last 100 years in the PiControl run, from 1861-2005 in the Historical run, and from 2006-2100 in RCP2.6, RCP4.5, RCP6.0, RCP8.5. The multi-model ensemble includes a total of 1800 years for the PiControl run, 2610 years in the Historical and 1710 years for all warming scenarios. 
Annual Ensemble GMSST with Linear Trend Removed

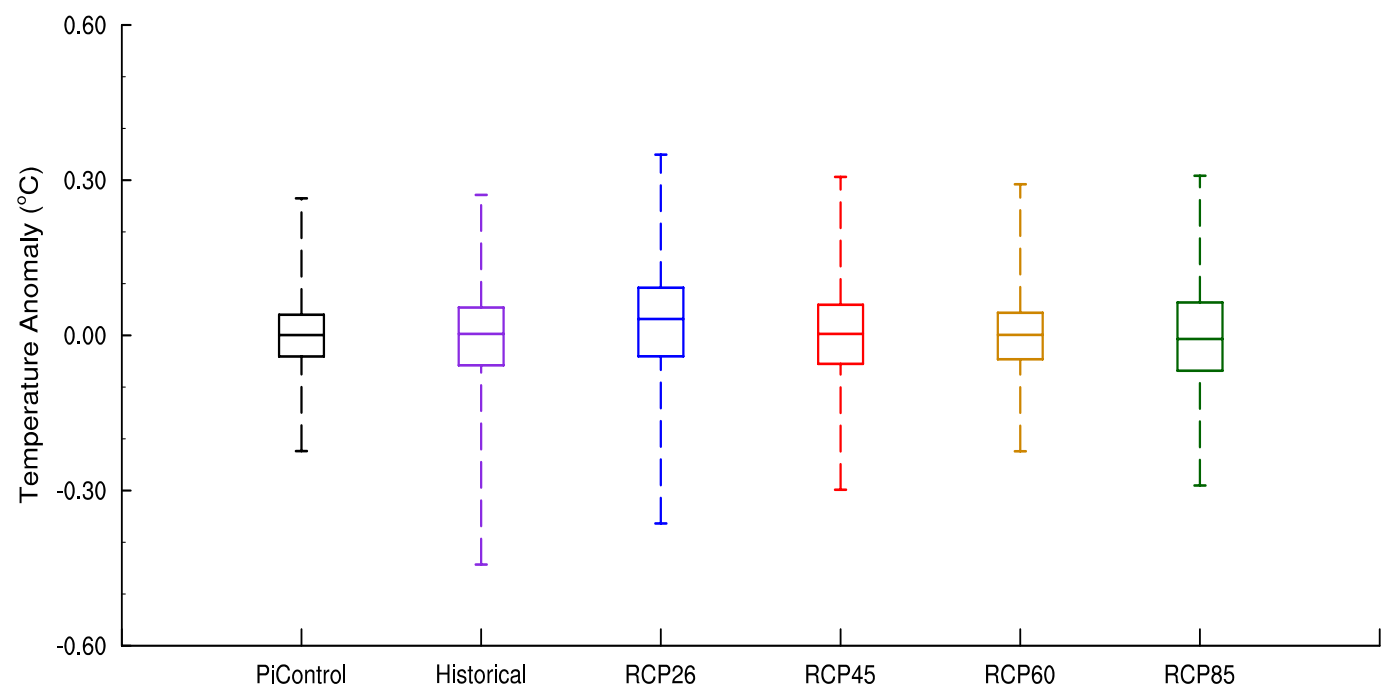

Figure 4.5: Box and whisker plots of the annually averaged multi-model ensemble GMSST after removing a least-squares linear trend for each individual ensemble member before concatenating. The multi-model ensemble consists of 19 ensemble members each detrended from the last 100 years in the PiControl run, from 1861-2005 in the Historical run, and from 2006-2100 in RCP2.6, RCP4.5, RCP6.0, RCP8.5. The multi-model ensemble includes a total of 1800 years for the PiControl run, 2610 years in the Historical and 1710 years for all warming scenarios. 


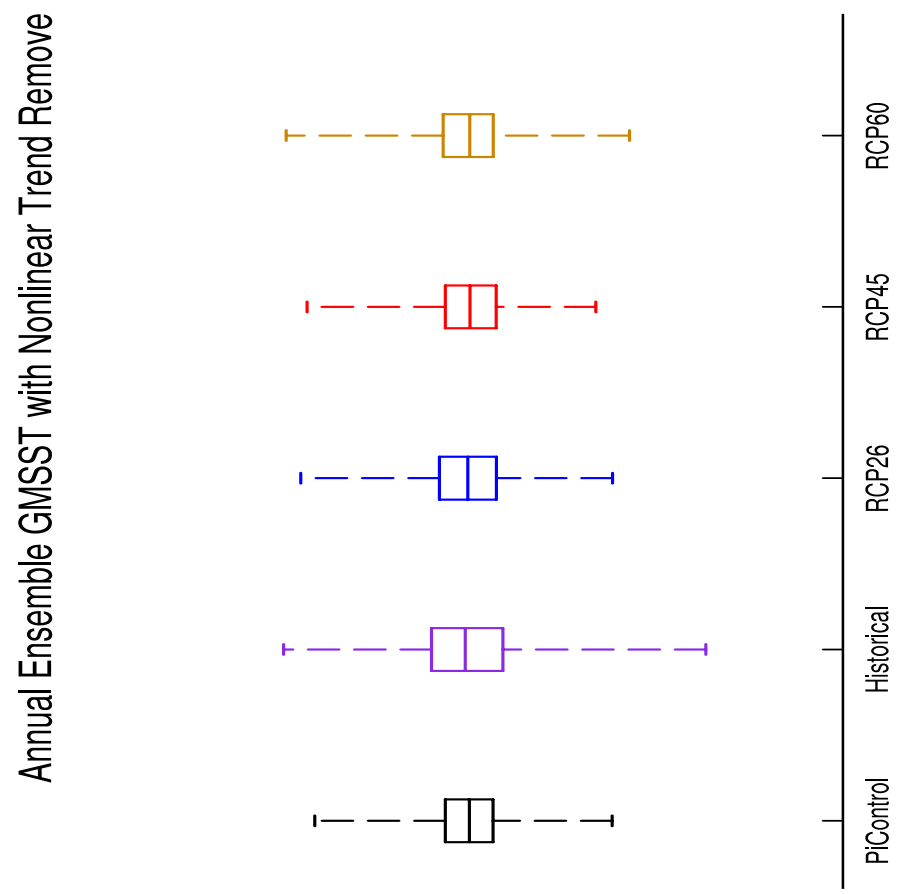

Figure 4.6: Box and whisker plots of the annually averaged multi-model ensemble GMSST after removing a least-squares quadratic trend for each individual ensemble member before concatenating. The multi-model ensemble consists of 19 ensemble members each detrended from the last 100 years in the PiControl run, from 1861-2005 in the Historical run, and from 2006-2100 in RCP2.6, RCP4.5, RCP6.0, RCP8.5. The multimodel ensemble includes a total of 1800 years for the PiControl run, 2610 years in the Historical and 1710 years for all warming scenarios. 


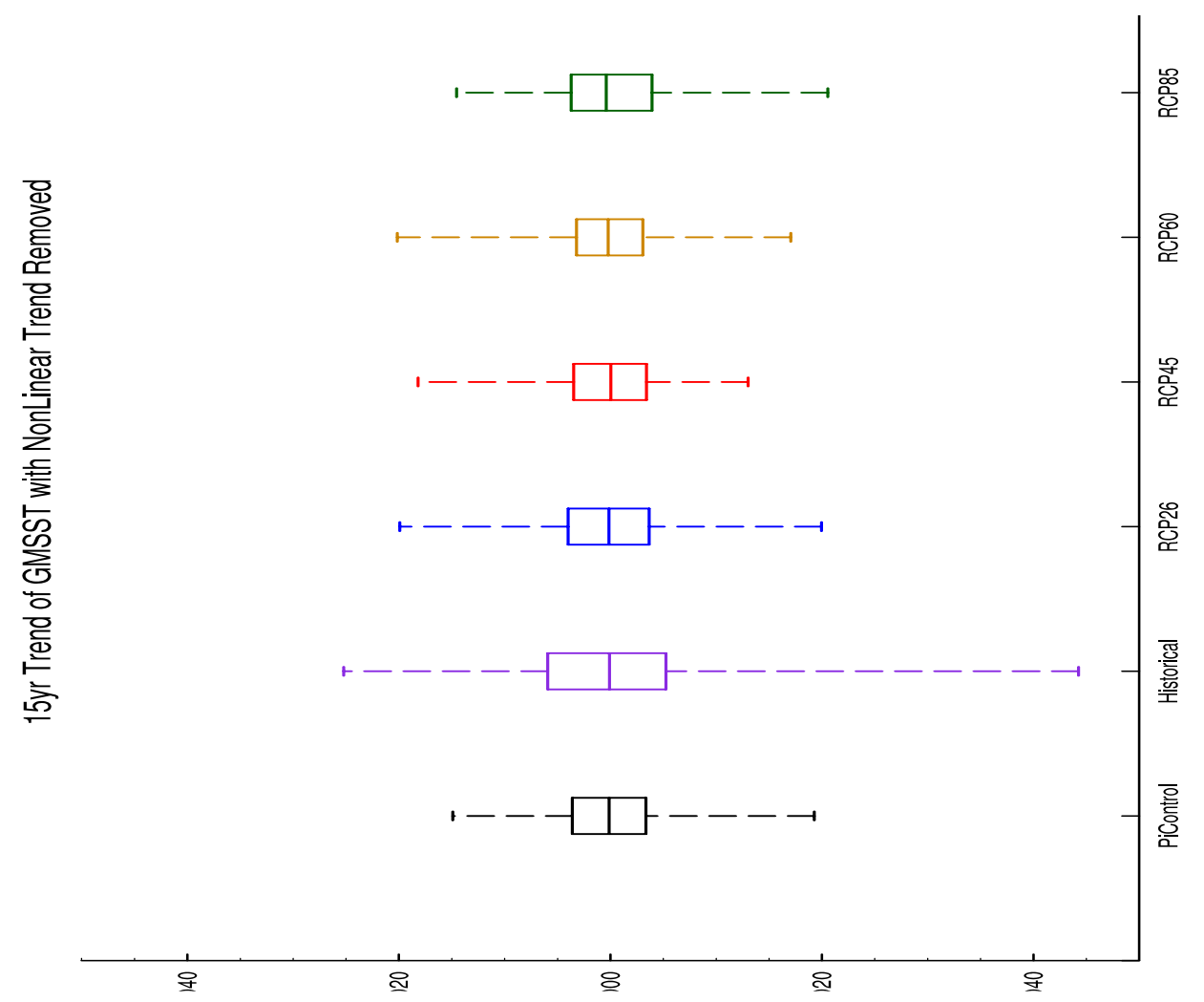

Figure 4.7: Box and whisker plots of the Monte Carlo sampled 15-year trends over the multi-model ensemble for the last 100 years in each PiControl model (1800 total simulated years), Historical from 1861-2005 (2610 years), and from 2006-2100 for RCP2.6, RCP4.5, RCP6.0, an RCP8.5 (1710 years for all warming scenarios) after removing a least squares quadratic trend from the individual ensemble members before concatenation. 


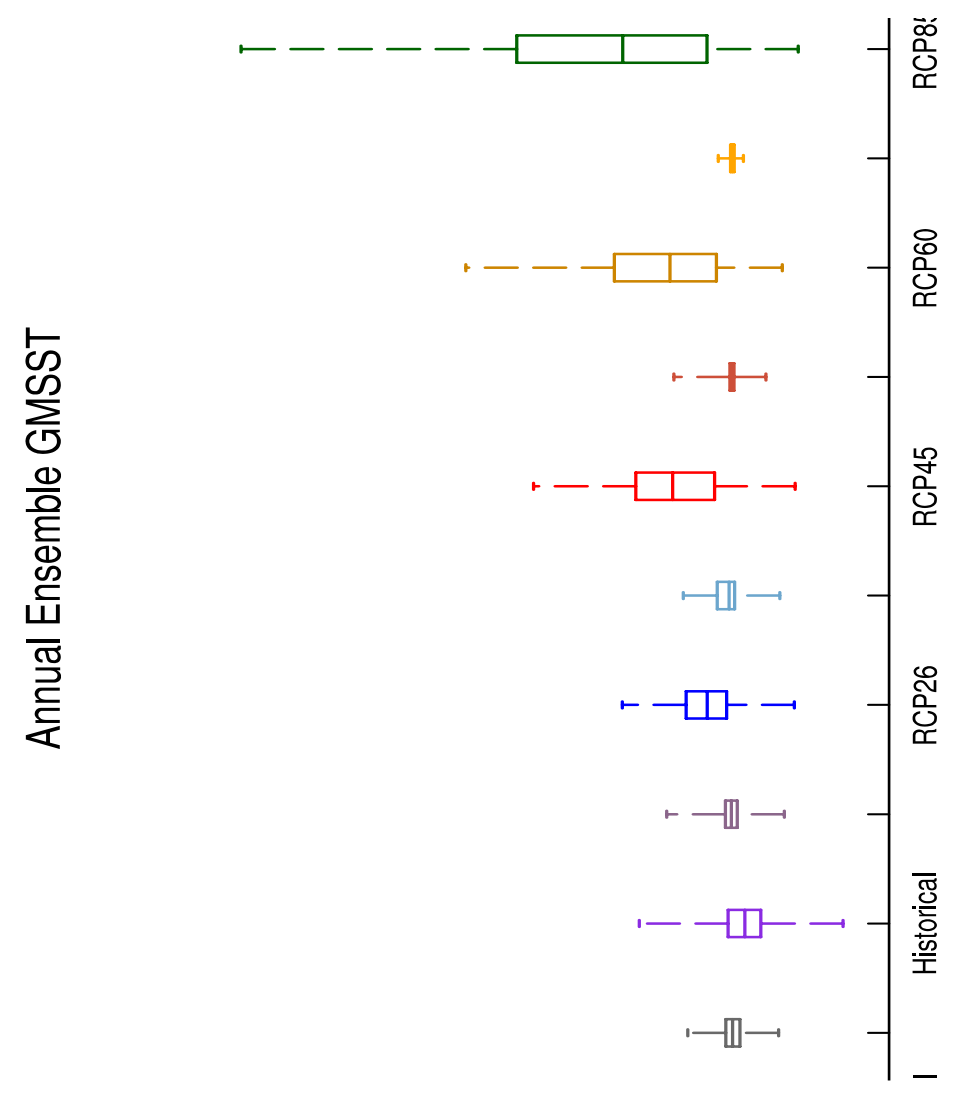

Figure 4.8: Box and whisker plots of the annually averaged multi-model ensemble GMSST alongside the multi-model GMSST from the EOF reconstruction with the largest trend removed. Both multi-model ensembles consist of 19 ensemble members each from the last 100 years in the PiControl run, from 1861-2005 in the Historical run, and from 2006-2100 in RCP2.6, RCP4.5, RCP6.0, and RCP8.5. The multi-model ensembles include a total of 1800 years for the PiControl run, 2610 years in the Historical and 1710 years for all warming scenarios. 


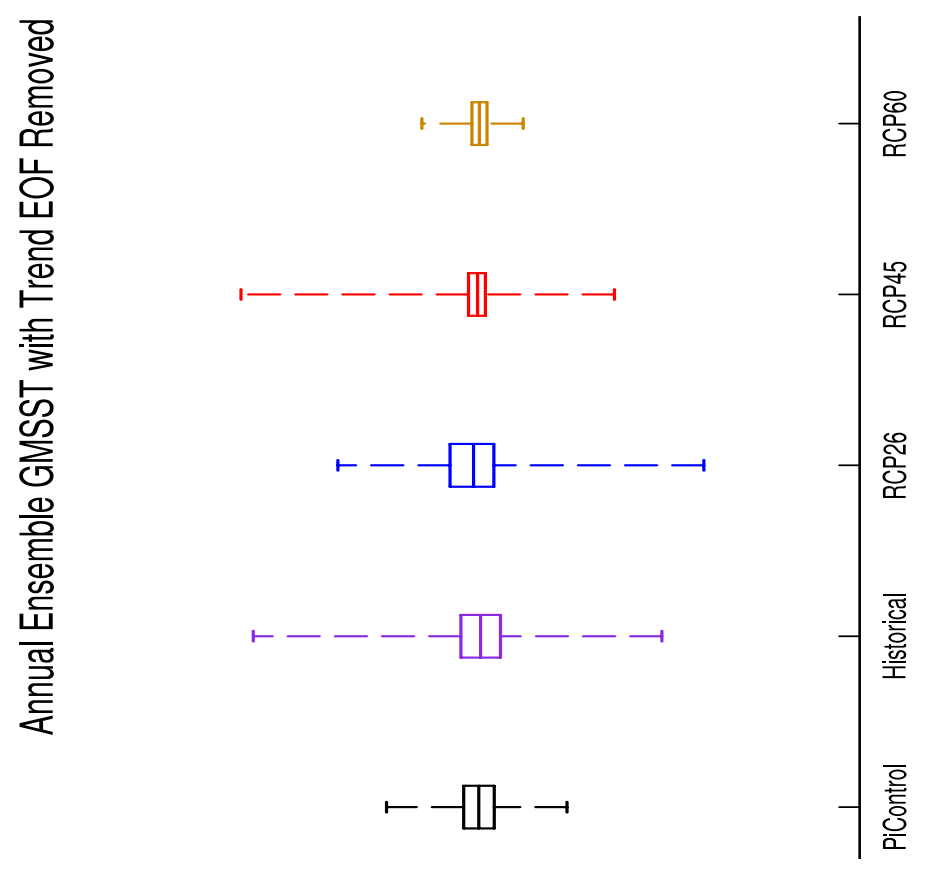

Figure 4.9: Box and whisker plots of the annually averaged multi-model GMSST from EOF reconstruction after the EOF with the largest trend is removed for the individual members before concatenating. The multi-model ensemble consists of 19 ensemble members each detrended from the last 100 years in the PiControl run, from 1861-2005 in the Historical run, and from 2006-2100 in RCP2.6, RCP4.5, RCP6.0, RCP8.5. The multimodel ensemble includes a total of 1800 years for the PiControl run, 2610 years in the Historical and 1710 years for all warming scenarios. 


\section{ERSST.v4 vs CMIP5 Models}
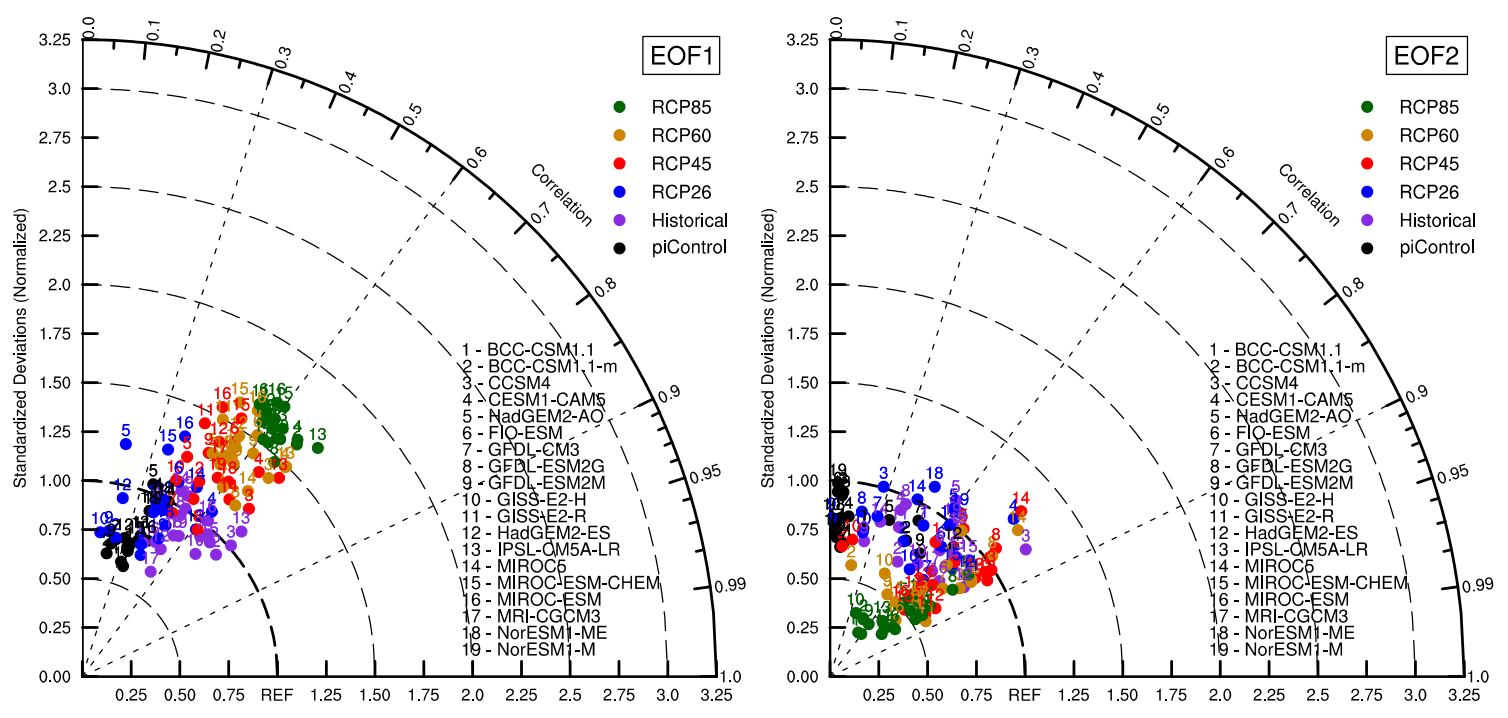

Figure 4.10: Taylor Diagram where each number on the diagram corresponds to an ensemble member and the colors denote the CMIP5 experiment the ensemble member is under. The radial distance from the origin to model member represents the ratio of the CMIP5 experiment to the observed ERSSTv4 standard deviation. The standard deviation is calculated using the explained variance for the respective EOF modes being compared. The azimuthal positions give the weighted centered pattern correlation for the CMIP5 first two EOF modes compared to the observed ERSSTv4 first two EOF modes. 

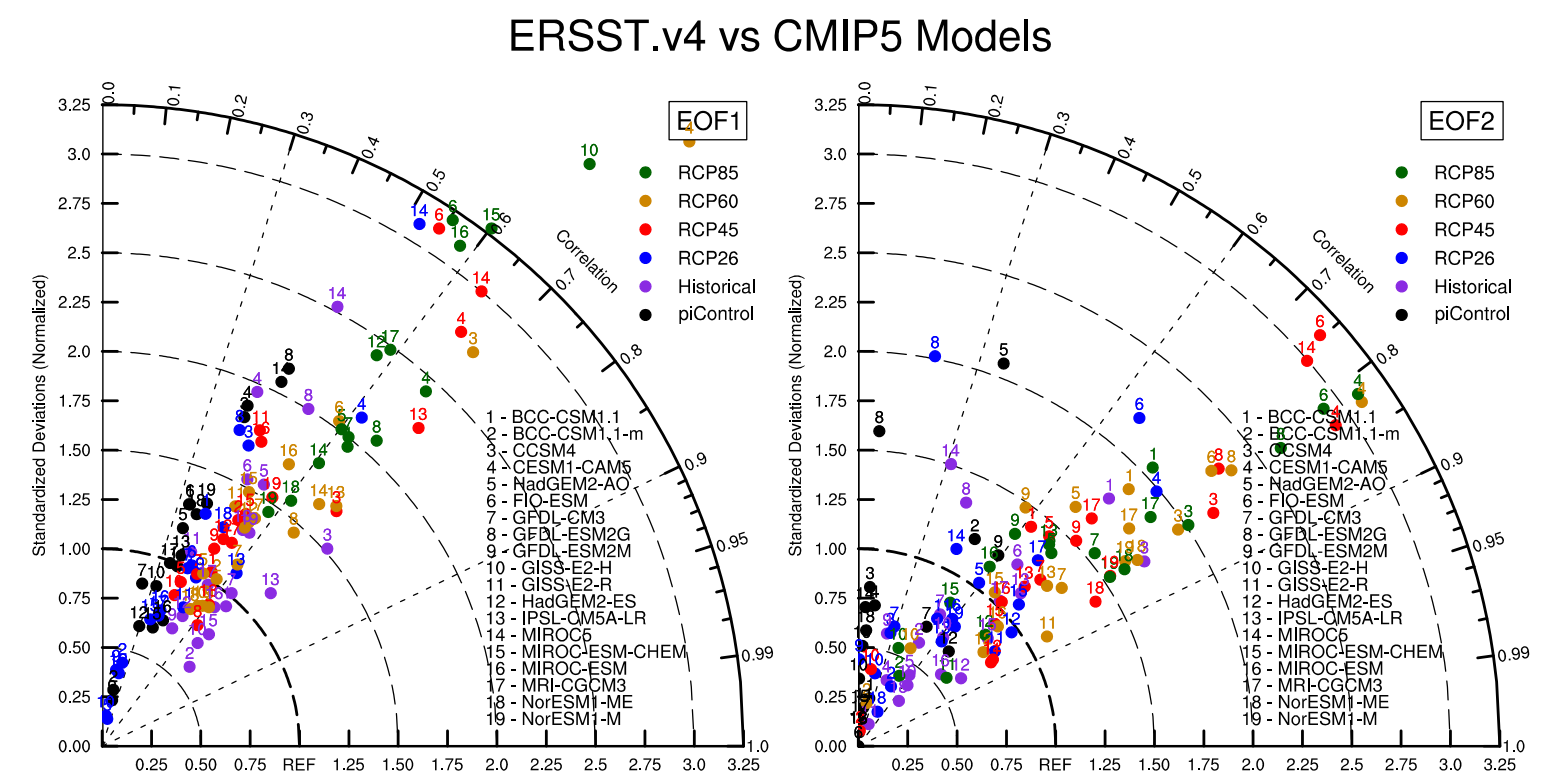

Figure 4.11: Taylor Diagram where each number on the diagram corresponds to an ensemble member and the colors denote the CMIP5 experiment the ensemble member is under. The radial distance from the origin to model member represents the ratio of the CMIP5 experiment to the observed ERSSTv4 standard deviation. The standard deviation is calculated using the global variance for the respective EOF modes being compared. The azimuthal positions give the weighted centered pattern correlation for the CMIP5 first two EOF modes compared to the observed ERSSTv4 first two EOF modes. 

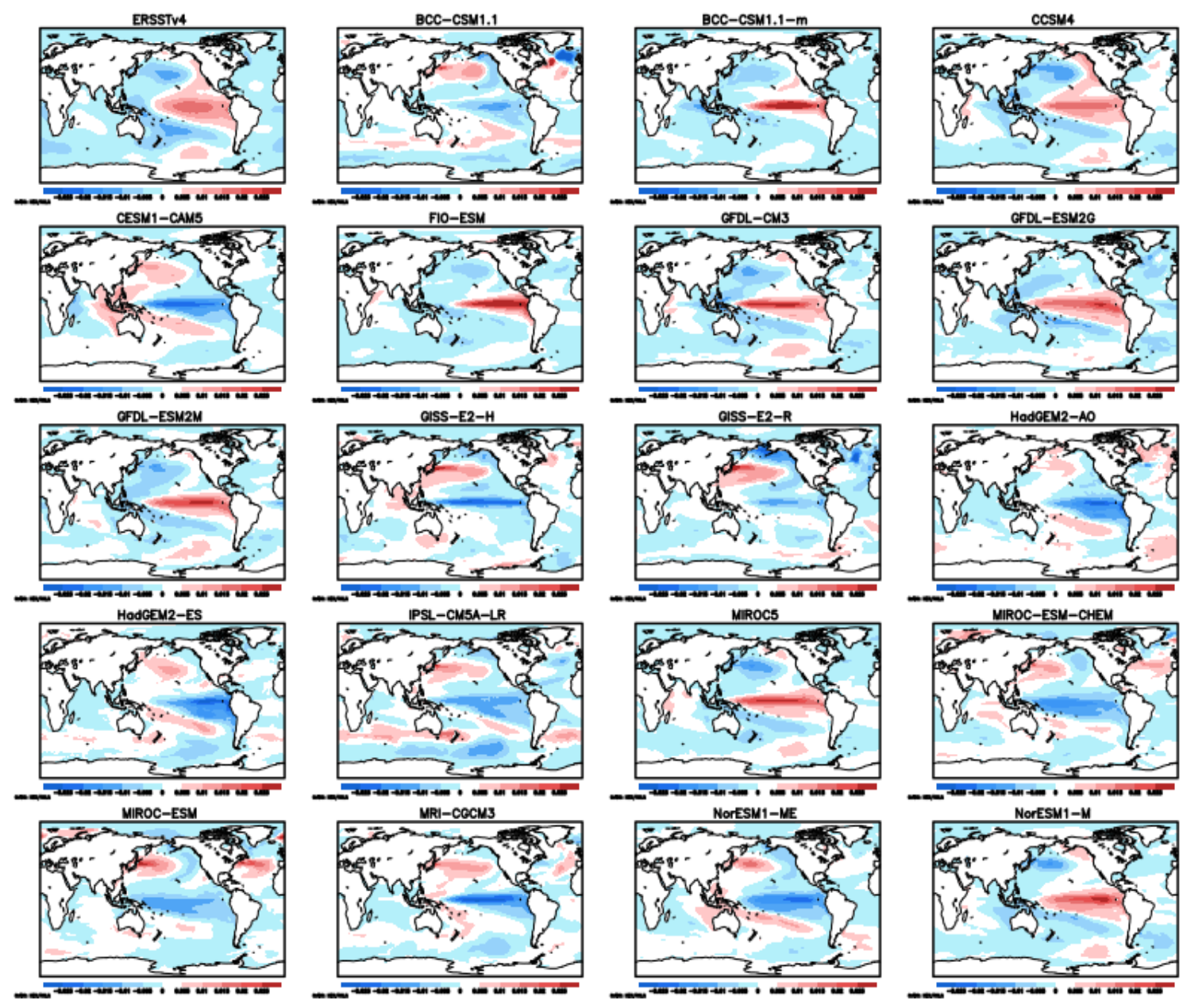

Figure 4.12: Spatial pattern of the second EOF for ERSSTV4 for the time period between 1850-2017 compared to the spatial pattern of the second EOF for 19 ensemble members under RCP8.5 radiative forcing from 2006-2100. 

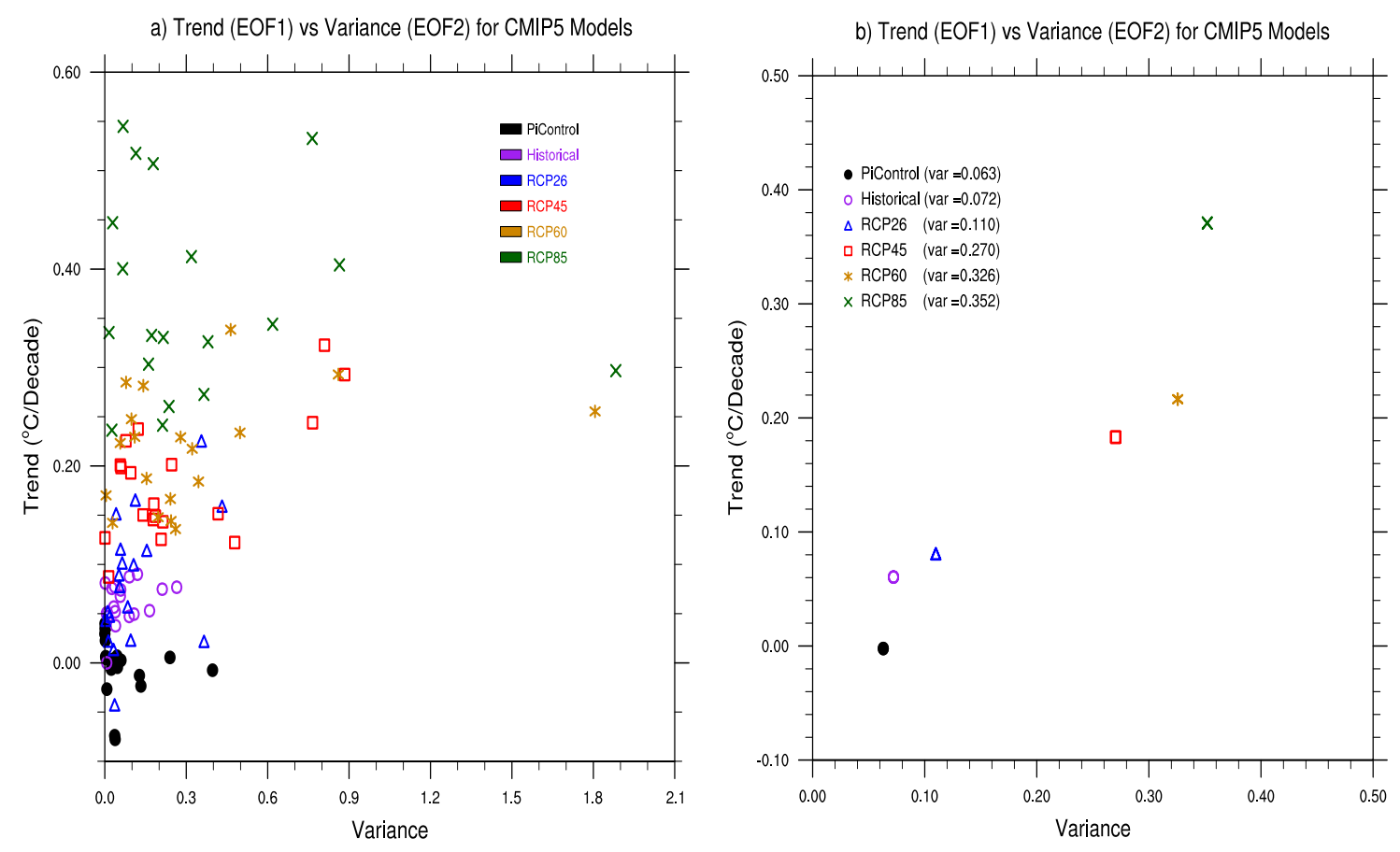

Figure 4.13: Scatter plot of linear trend per decade calculated using the trend found in the first EOF vs the global variance calculated in the second EOF for a) the individual model members and $b$ ) the multi-model ensemble average. 


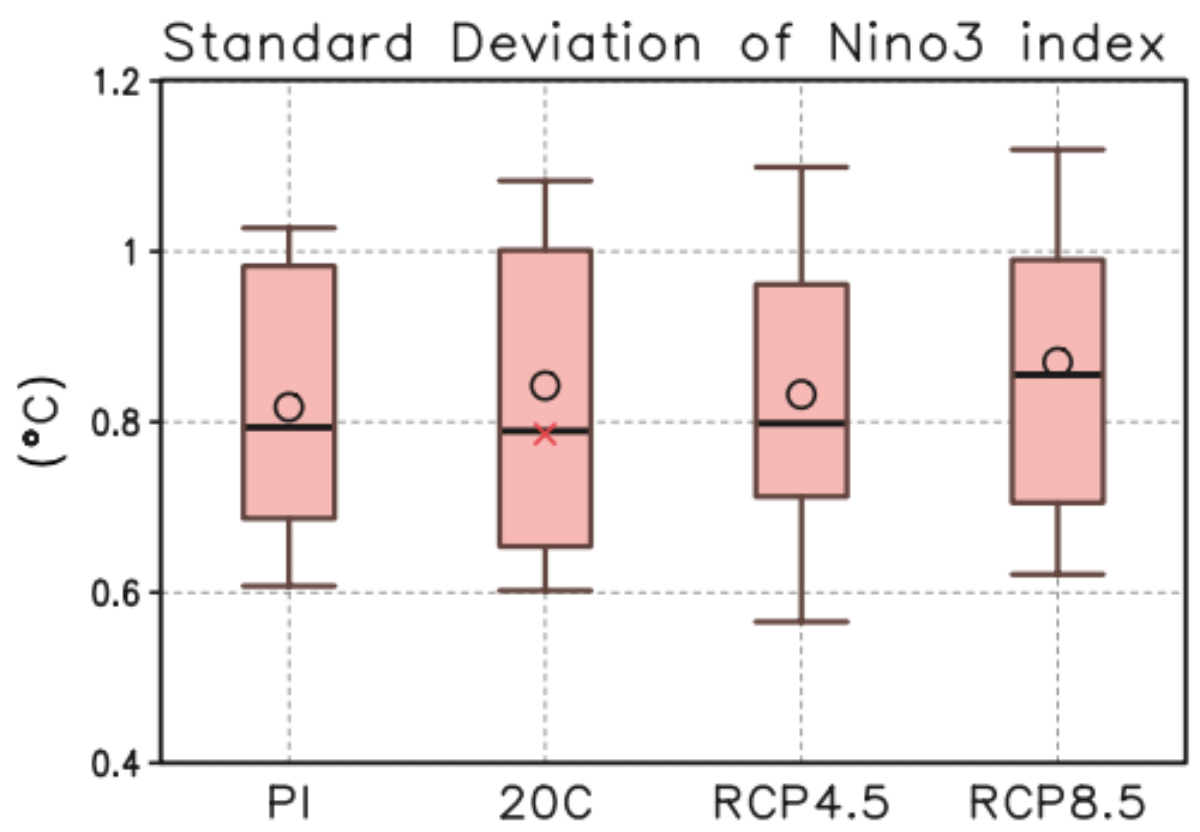

Figure 4.14: Taken from the IPCC report (2013); Standard deviation in CMIP5 multimodel ensembles of sea surface temperature variability over the eastern equatorial Pacific Ocean (Nino3 region: $5^{\circ} \mathrm{S}-5^{\circ} \mathrm{N}, 150^{\circ} \mathrm{W}-90^{\circ} \mathrm{W}$ ), a measure of $\mathrm{El}$ Nino amplitude, for the pre-industrial (PI) control and 20th century (20C) simulations, and 21st century projections using RCP4.5 and RCP8.5. Thirty-one models are used for the ensemble average. Open circles indicate multi-model ensemble means, and the red cross symbol is the observed standard deviation for January 1870 - December 2011 obtained from HadISSTv1. The linear trend and climatological mean of seasonal cycle have been removed. Box-whisker plots show the 16th, 25th, 50th, 75th, and 84th percentiles. 


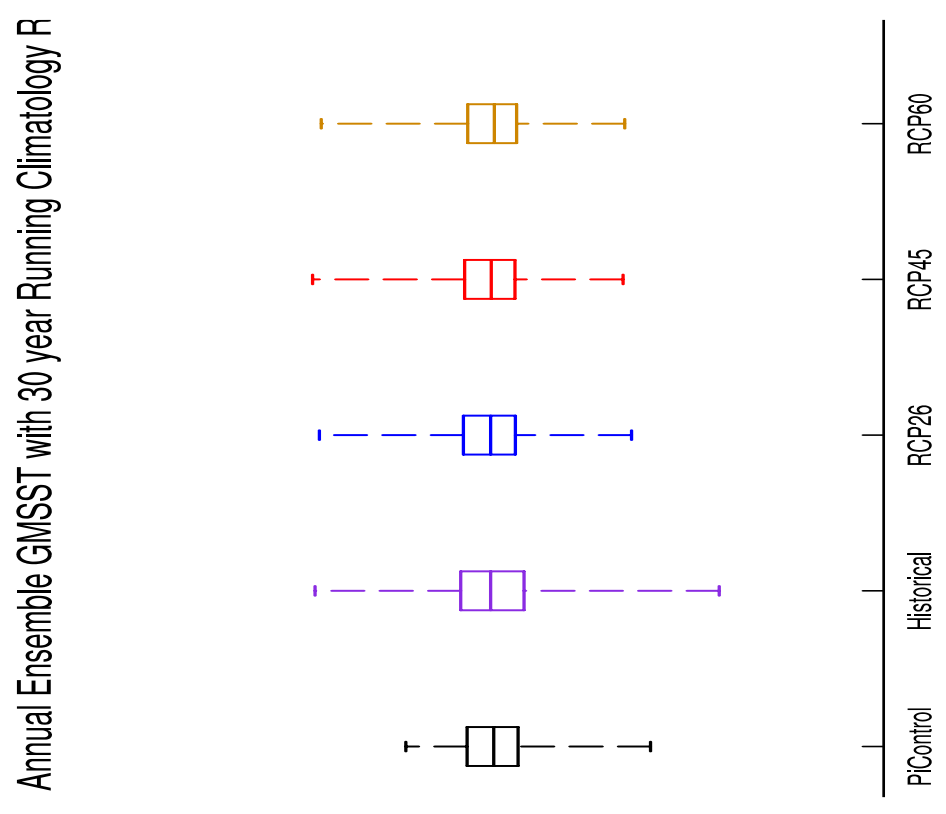

Figure 4.15: Box and whisker plots of the annually averaged multi-model ensemble GMSST with 30-year running climatology removed. The multi-model ensemble consists of 19 ensemble members each detrended from the last 100 years in the PiControl run, from 1861-2005 in the Historical run, and from 2006-2100 in RCP2.6, RCP4.5, RCP6.0, RCP8.5. The multi-model ensemble includes a total of 1260 years for the PiControl run, 2070 years in the Historical and 1170 years for all warming scenarios. 


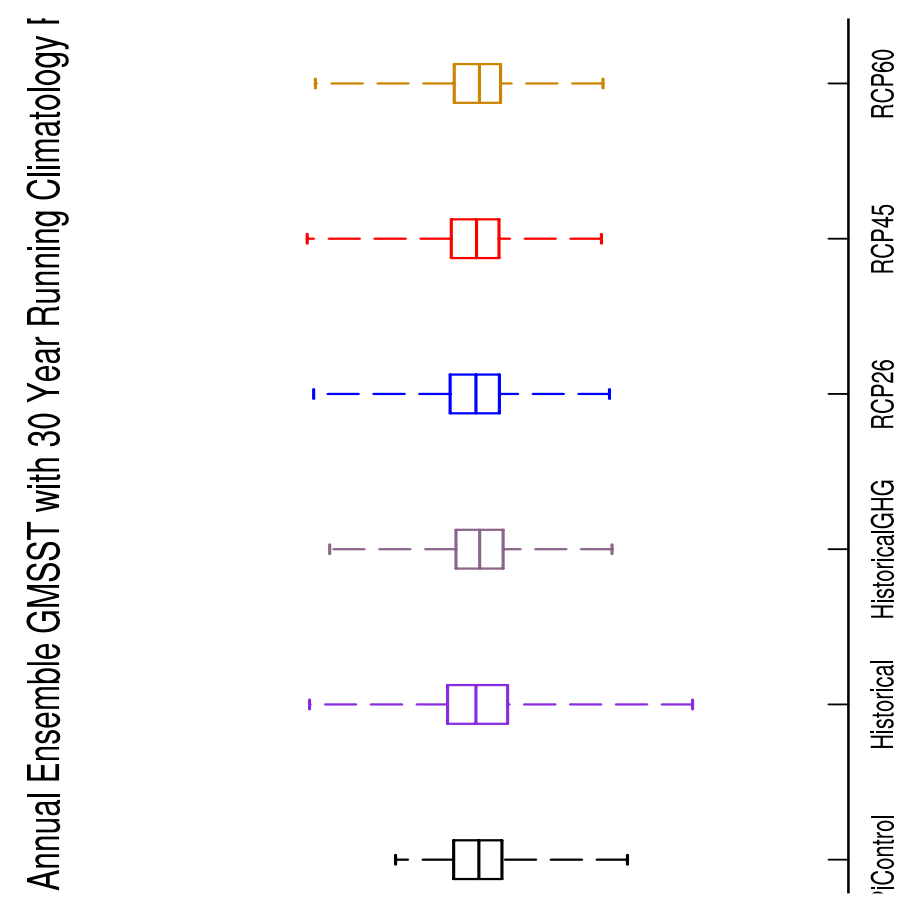

Figure 4.16: Box and whisker plots of the annually averaged multi-model ensemble GMSST with 30 year running climatology removed for the PiControl (1260 years), Historical (2070 years), a Historical greenhouse gas only run (1885 year), and RCP2.6, RCP4.5, RCP6.0, RCP8.5 (1170 years for all warming scenarios). 
Trend vs Variance for CMIP5 Models

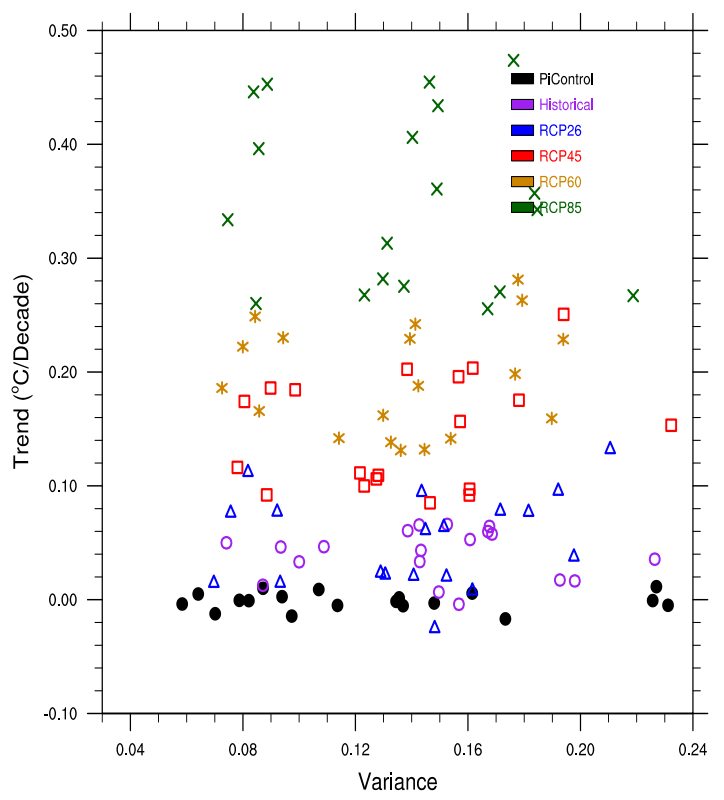

Trend vs Variance for CMIP5 Models

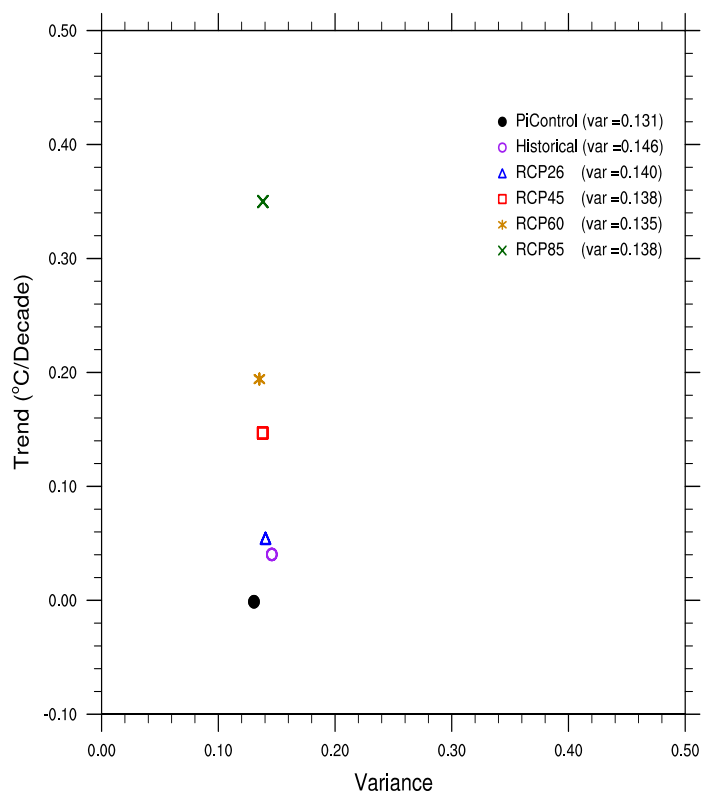

Figure 4.17: Scatter plot of linear trend per decade vs variance of each model after removing 30 year running mean for the individual model members on the left and the multi-model ensemble average on the right. 
a) Trend vs. Varaiance: Atlantic Ocean
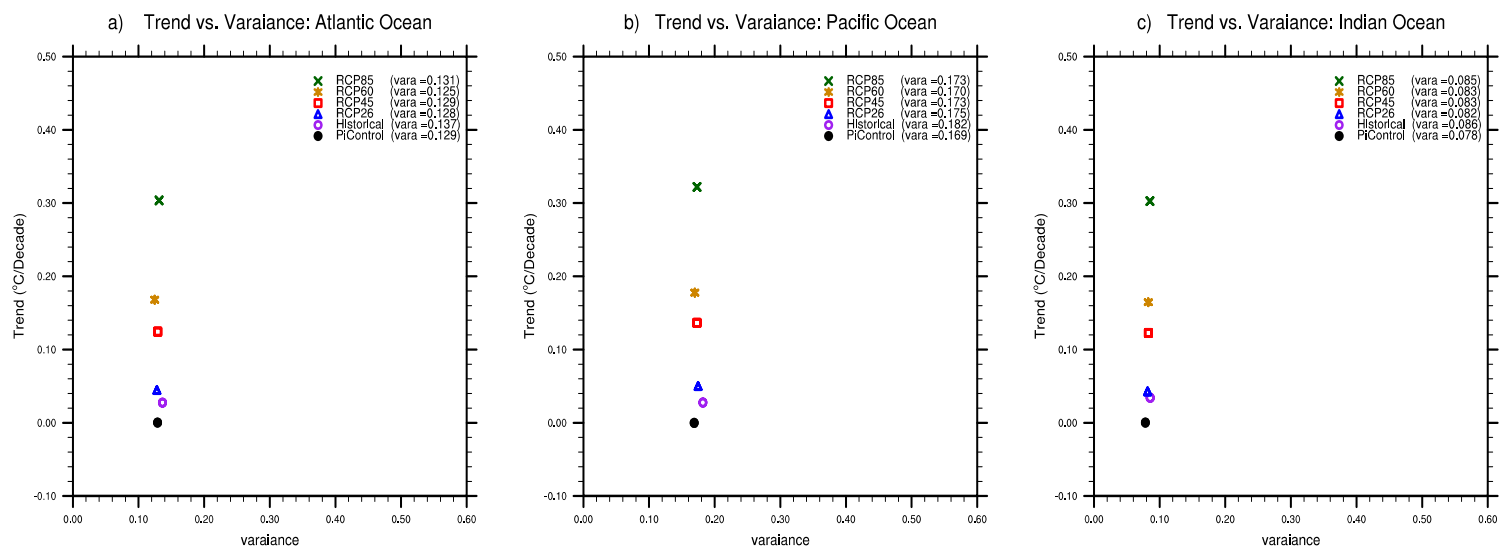

d) Trend vs. Varaiance: Southern Ocean
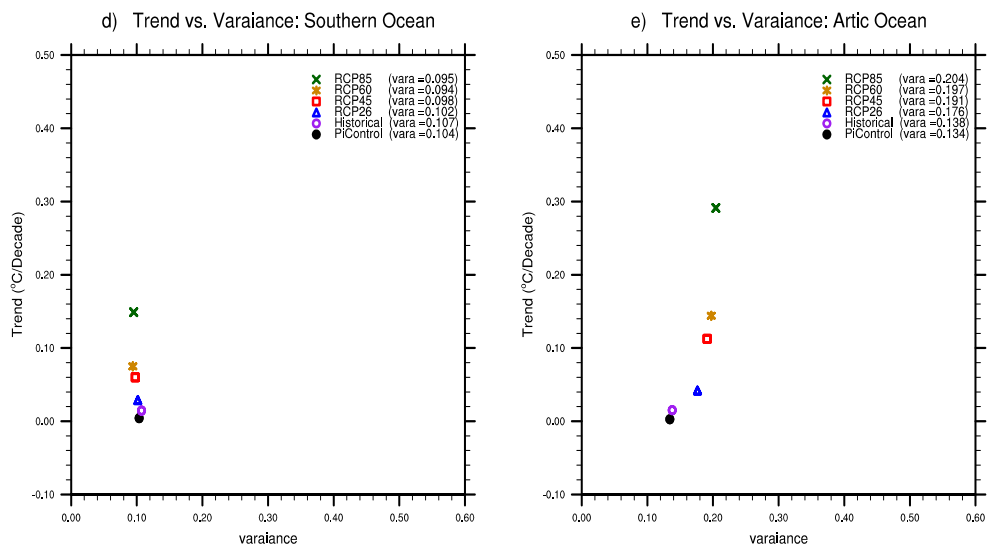

Figure 4.18: Scatter plot of the multi-model ensemble average linear trend per decade calculated from the raw SST data in the a) Atlantic Ocean b) Pacific Ocean c) Indian Ocean d) Southern Ocean e) Artic Ocean vs variance calculated in each basin for 19 models for PiControl, Historical, RCP2.6, RCP4.5, RCP6.0, and RCP8.5. 

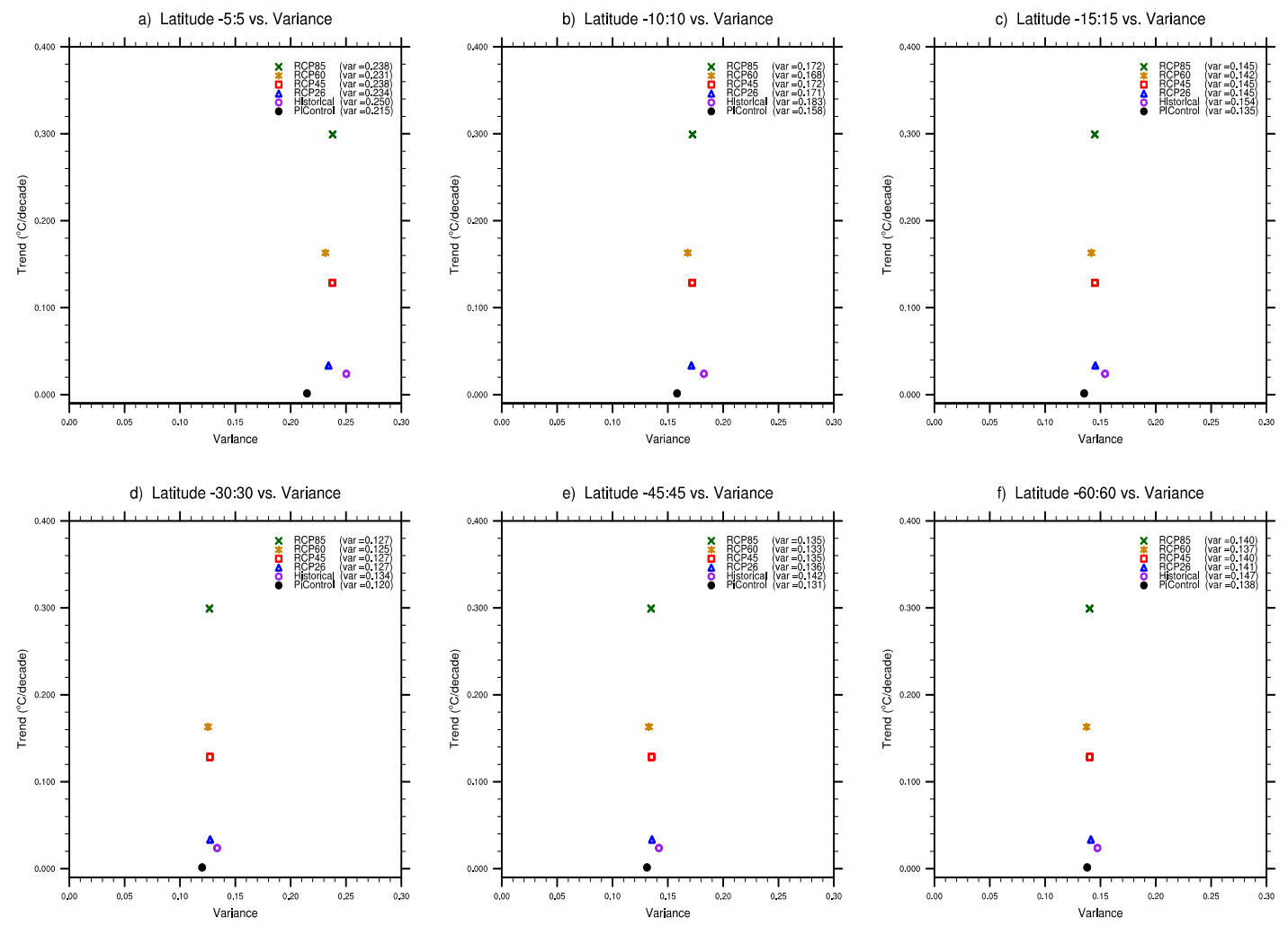

Figure 4.19: Scatter plot of the multi-model ensemble average linear trend per decade vs variance for 19 models for PiControl, Historical, RCP2.6, RCP4.5, RCP6.0, and RCP8.5 by a) latitude $5 \mathrm{~S}$ to $5 \mathrm{~N}$ b) latitude $10 \mathrm{~S}$ to $10 \mathrm{~N}$ c) latitude $15 \mathrm{~S}$ to $15 \mathrm{~N}$ d) latitude $30 \mathrm{~S}$ to $30 \mathrm{~N}$ e) latitude $45 \mathrm{~S}$ to $45 \mathrm{~N}$ f) latitude $60 \mathrm{~S}$ to $60 \mathrm{~N}$. 

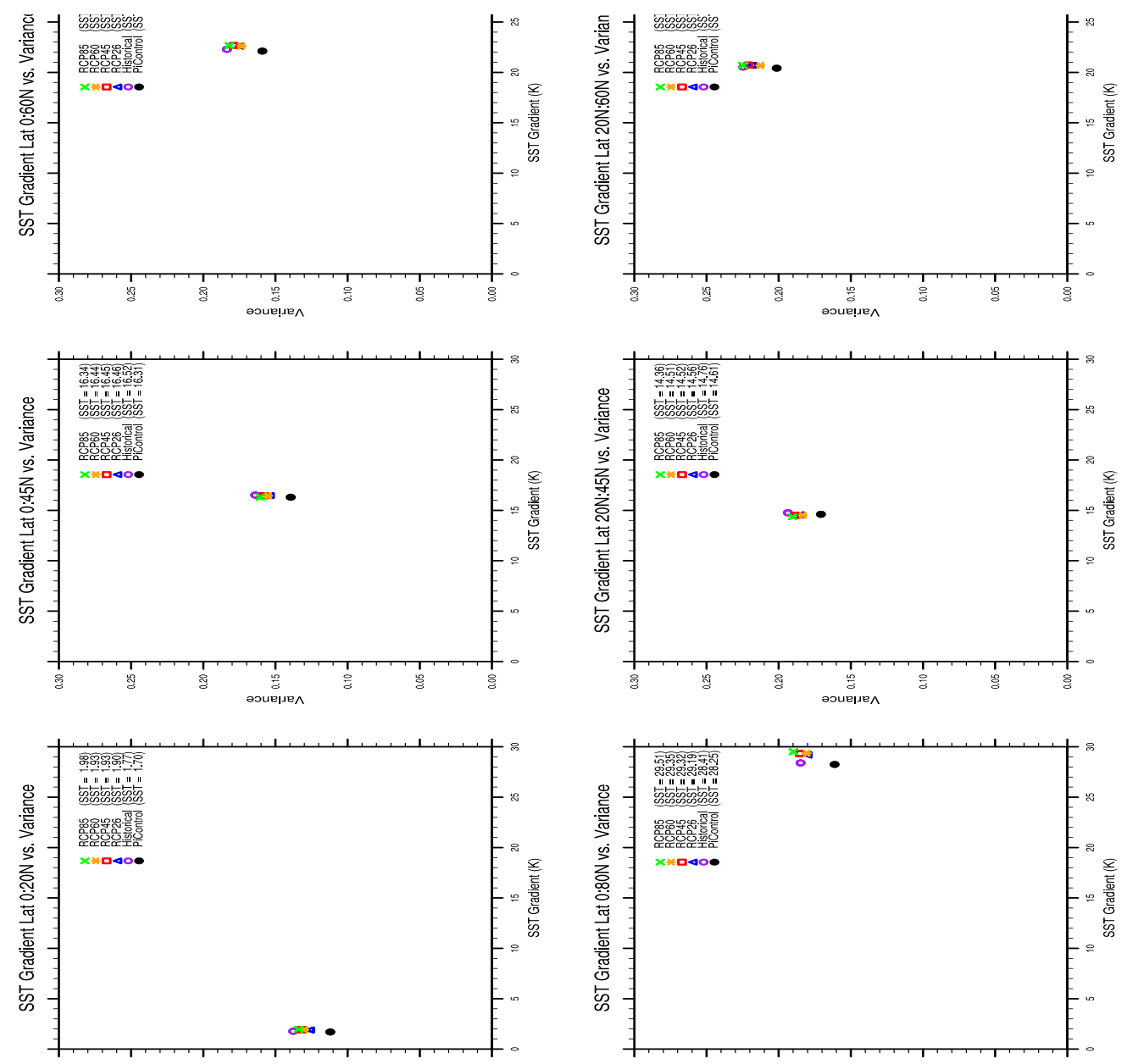

Figure 4.20: Scatter plot of ensemble variance vs sea surface temperature gradient for 19 models for PiControl, Historical, RCP2.6, RCP4.5, RCP6.0, and RCP8.5 by latitude a) 0$20 \mathrm{~N} \mathrm{b)} 0-45 \mathrm{~N}$ c) $0-60 \mathrm{~N} \mathrm{~d}) 0: 80 \mathrm{~N}$ e) $20 \mathrm{~N}-45 \mathrm{~N}$ f) $20 \mathrm{~N}-60 \mathrm{~N}$. 

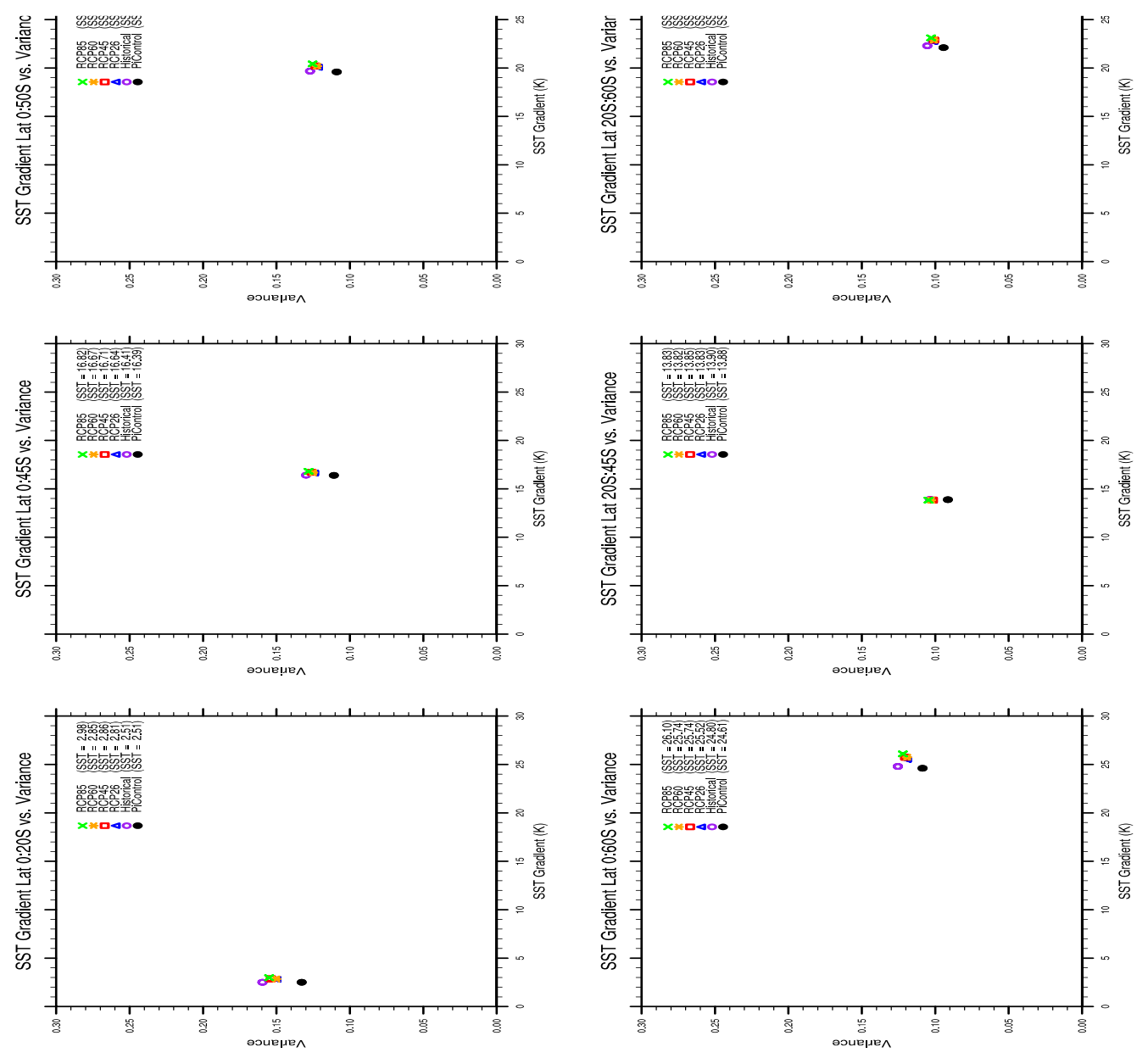

Figure 4.21: Scatter plot of ensemble variance vs sea surface temperature gradient for 19 models for PiControl, Historical, RCP2.6, RCP4.5, RCP6.0, and RCP8.5 by latitude a) 0$20 \mathrm{~S} \mathrm{b)} 0-45 \mathrm{~S}$ c) $0-50 \mathrm{~S}$ d) $0: 60 \mathrm{~S}$ e) $20 \mathrm{~S}-45 \mathrm{~S} \mathrm{f}) 20 \mathrm{~S}-60 \mathrm{~S}$. 
a) Variance Ratio: (RCP2.6/Historical)

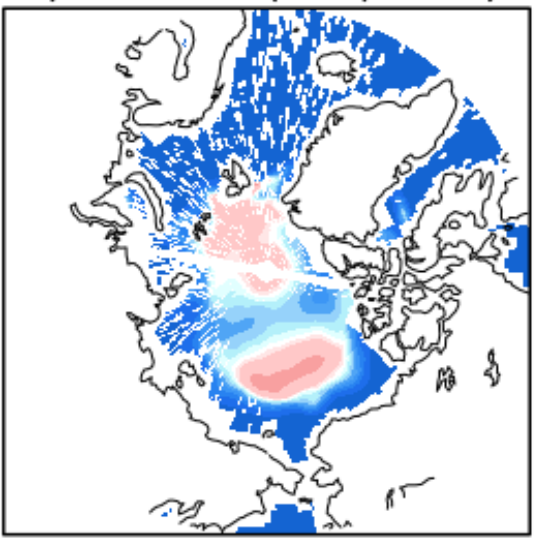

c) Variance Ratio: (RCP6.0/Historical)

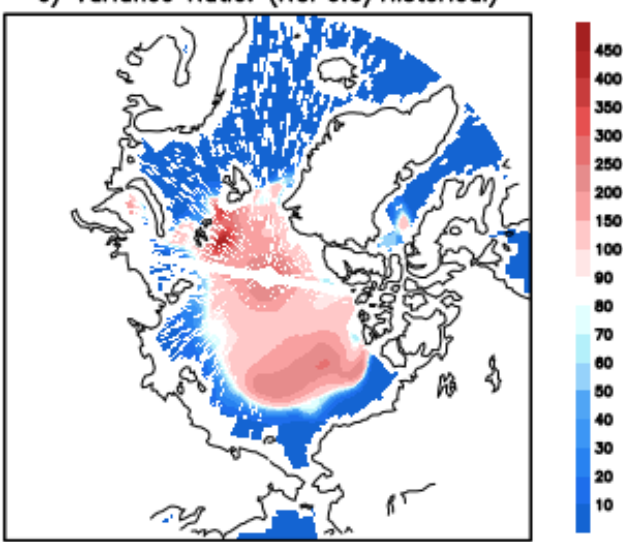

b) Variance Ratio: (RCP4.5/Historical)

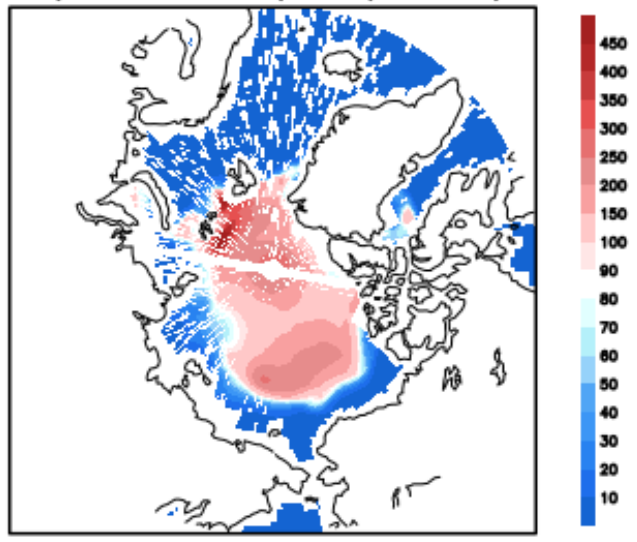

d) Variance Ratio: (RCP8.5/Historical)

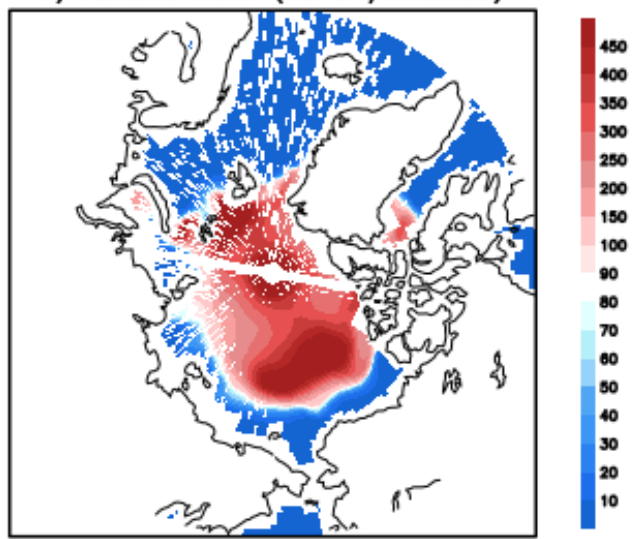

Figure 4.22: Ratio of two variances of SSTA to the Historical run in the Artic between the a) RCP26 simulation, b) RCP45 simulation, c) RCP60 simulation, d) RCP85 simulation. 
a) Voriance Ratio: (RCP2.6/Historical)
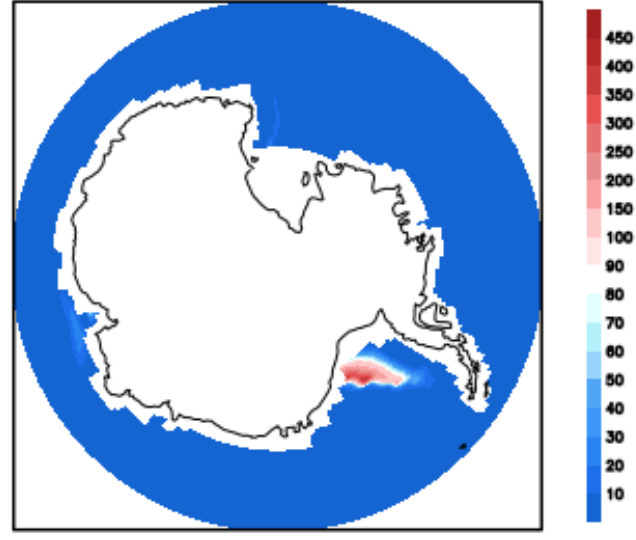

c) Variance Ratio: (RCP6.0/Historical)

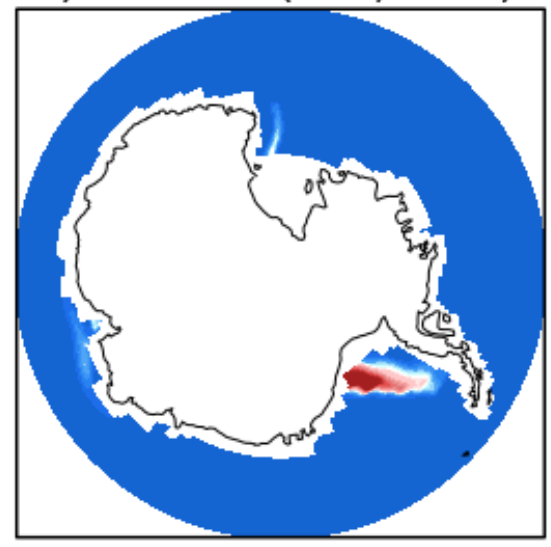

b) Variance Ratio: (RCP4.5/Historical)

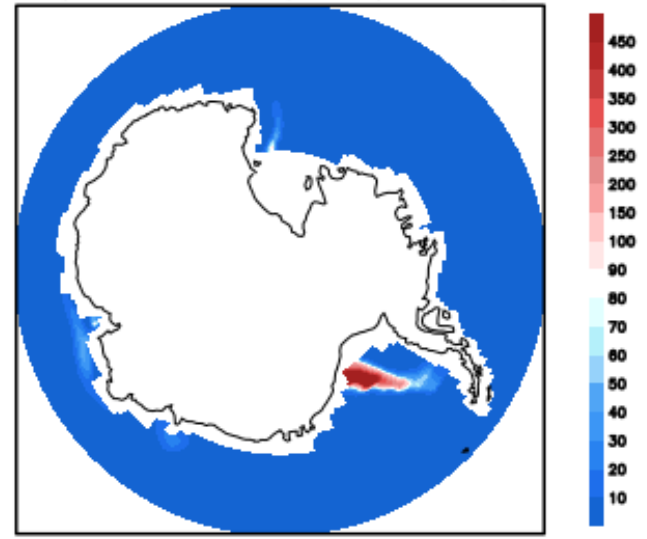

d) Variance Ratio: (RCP8.5/Historical)

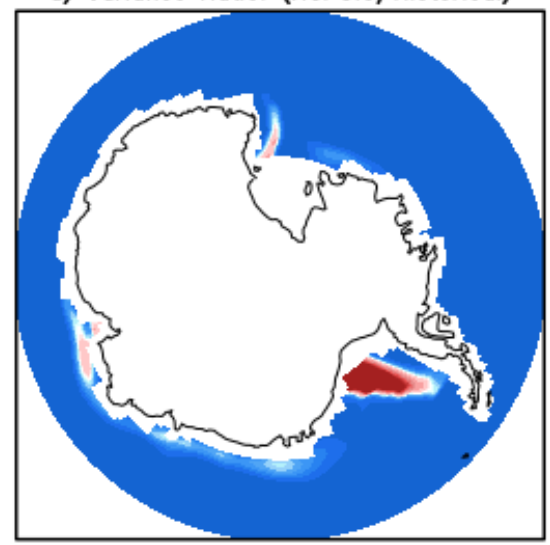

Figure 4.23: Ratio of two variances of SSTA to the Historical run in the Antarctic between the a) RCP26 simulation, b) RCP45 simulation, c) RCP60 simulation, d) RCP85 simulation. 
a) Variance Ratio: (RCP2.6/Historical)

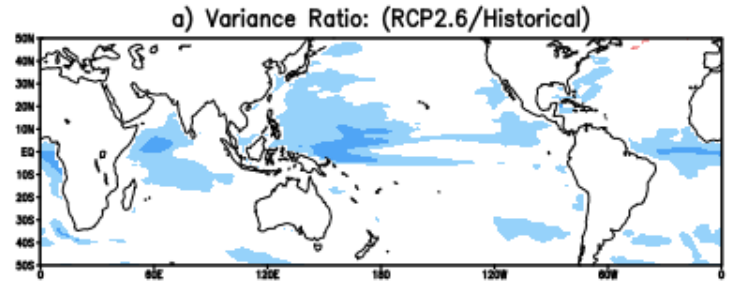

$\begin{array}{llll}0.5 & 0.7 & 0.8 & 0.8\end{array}$
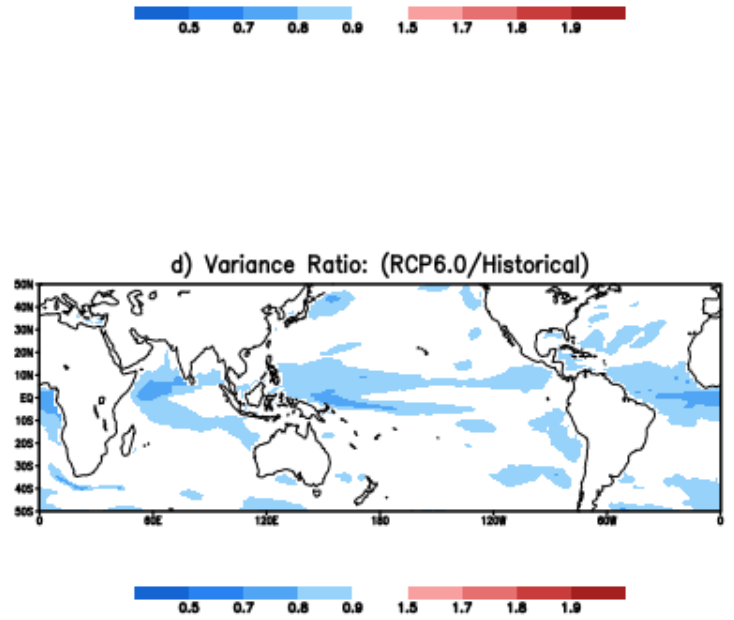

b) Variance Ratio: (RCP4.5/Historical)

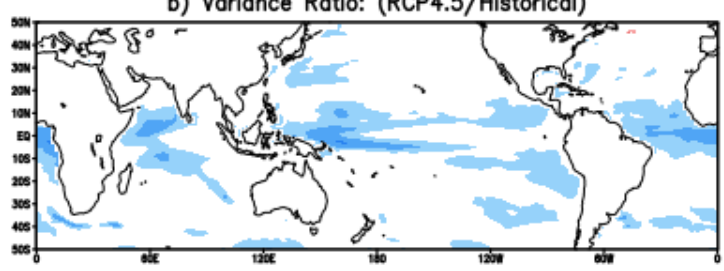

$\begin{array}{llll}0.5 & 0.7 & 0.8 & 0.9\end{array}$
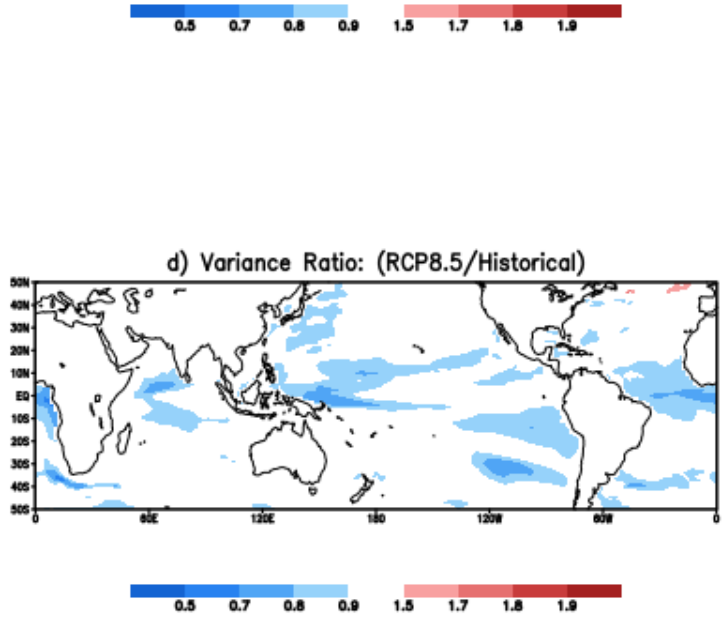

Figure 4.24: Ratio of two variances of SSTA to the Historical run from $60 \mathrm{~S}-60 \mathrm{~N}$ in the a) RCP26 simulation, b) RCP45 simulation, c) RCP60 simulation, d) RCP85 simulation. Blue shading denotes decreases in variance and Red indicated increases in variance. 

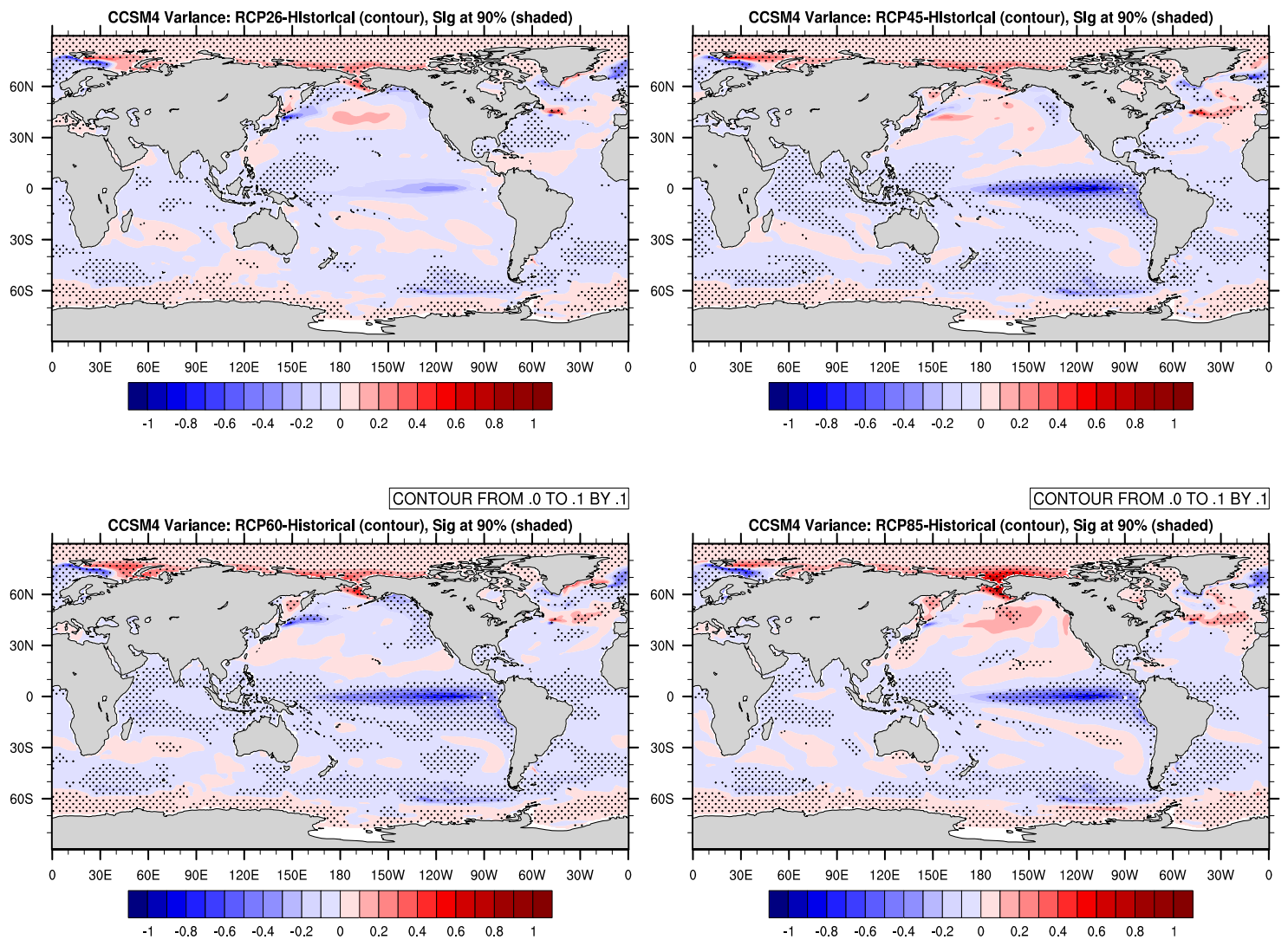

CONTOUR FROM .0 TO $1 \mathrm{BY} .1$

CONTOUR FROM .0 TO 1 BY.1

Figure 4.25: Variance difference by RCP scenario from Historical scenario for CCSM4 (contoured). Red denotes an increase in variance and Blue denotes a decrease in variance. Hatching indicates statistically significant areas at $90 \%$ significance level. 

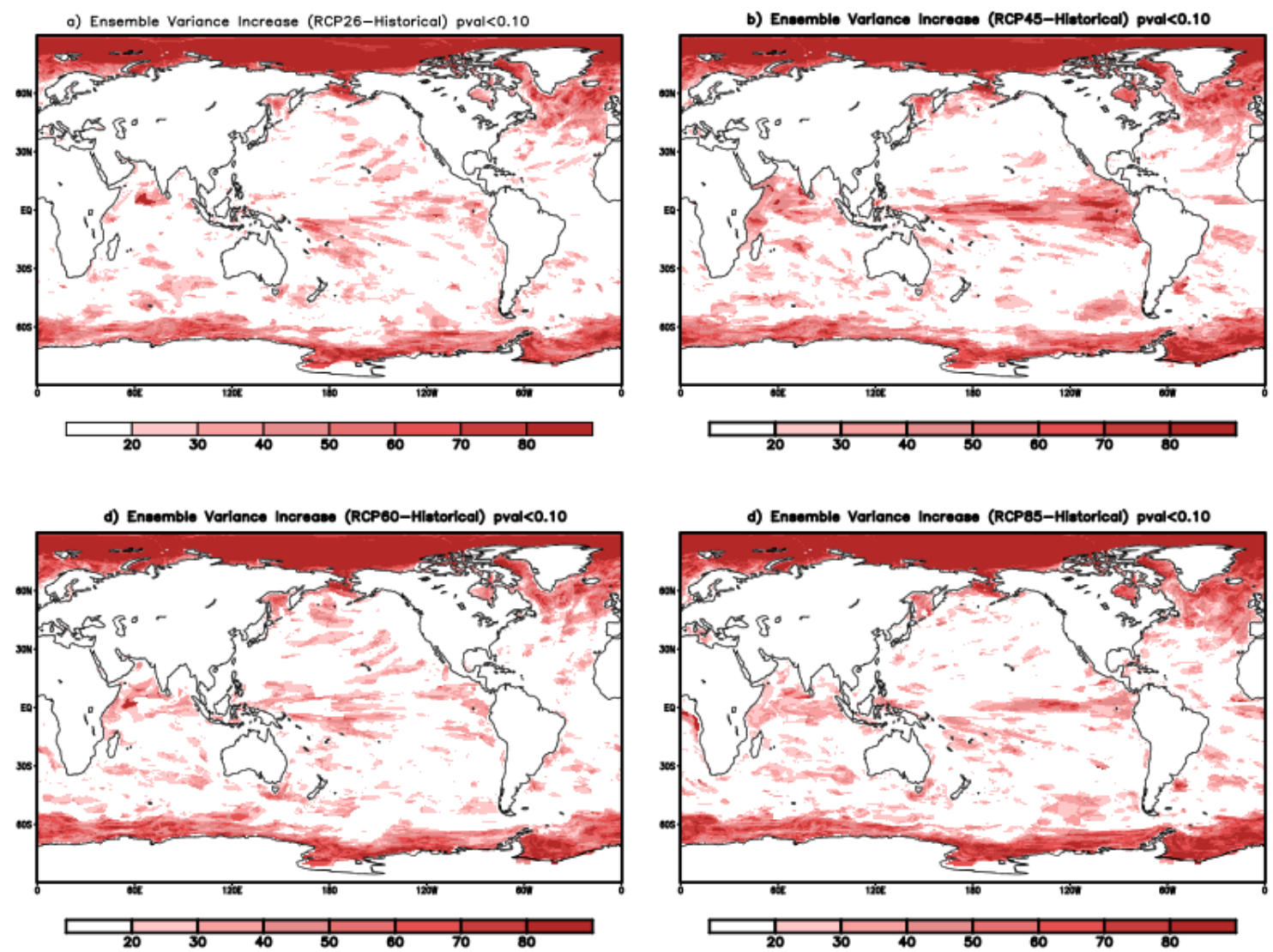

Figure 4.26: Percentage of ensemble members predicting an increase in variance that is statistically significant $(\mathrm{p}<0.10)$ from a) RCP26-Historical b) RCP45-Historical, c) RCP60-Historical, and d) RCP85-Historical. 
a) Ensemble Variance Decrease (RCP26-Historical) pva1<0.10

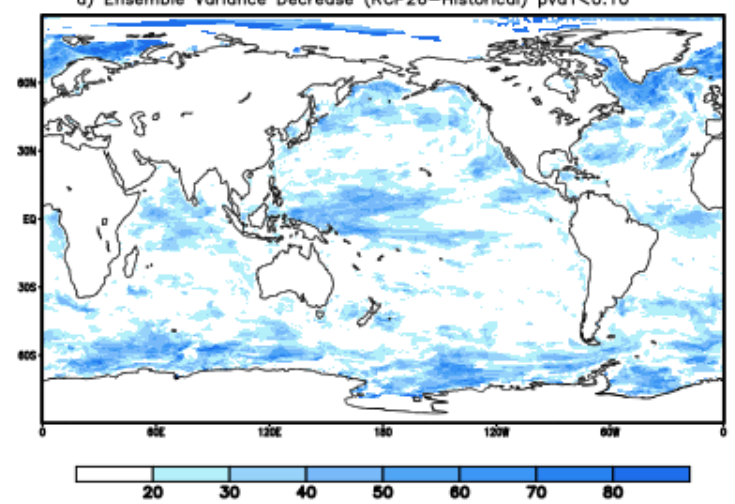

c) Ensemble Variance Decrease (RCP60-Hletorical) pval<0.010

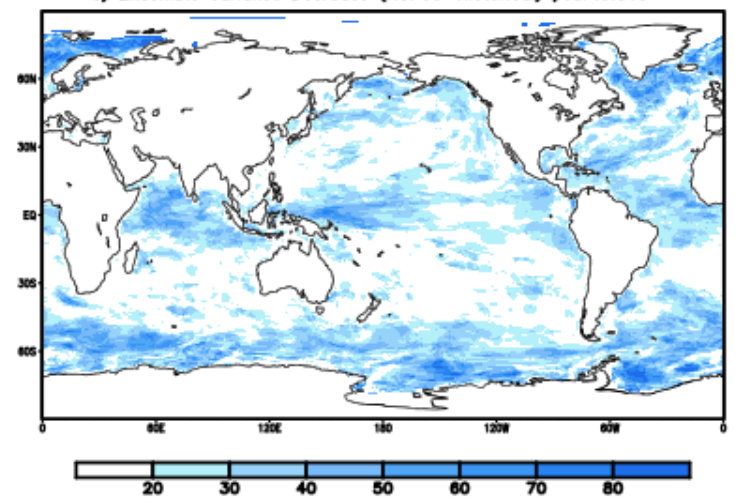

b) Ensemble Vorlance Decrease (RCP45-Hatorleal) pva1<0.10

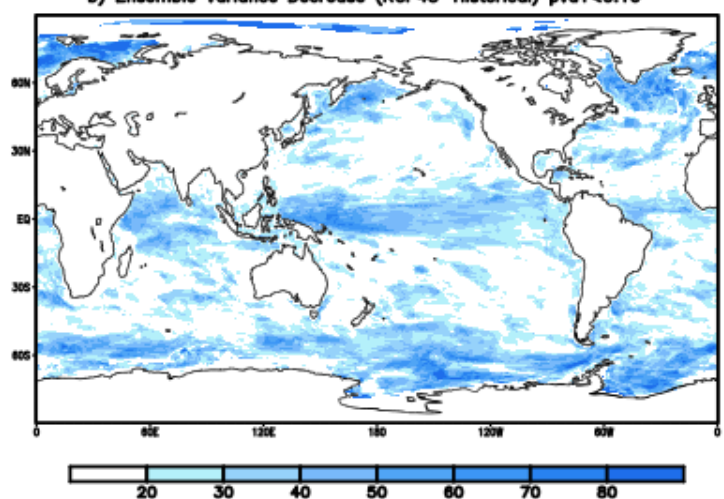

d) Ensemble Varlance Decrease (RCP85-Hletorleal) pva1<0.10

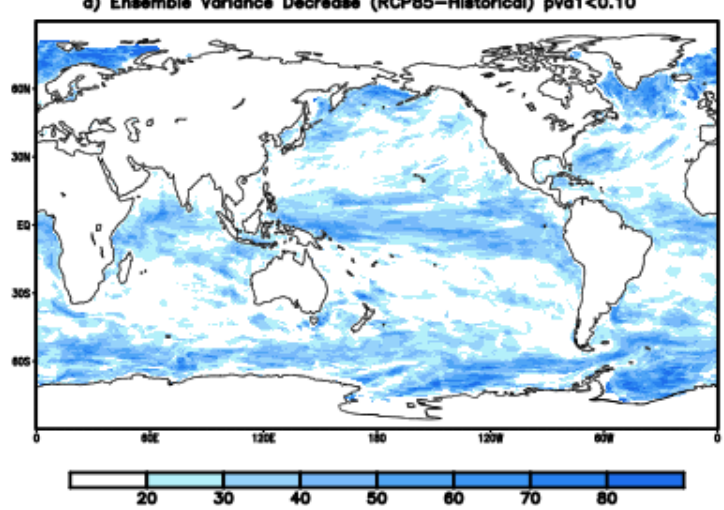

Figure 4.27: Similar to Figure 4.25 but denoting the percent of ensemble members predicting a decrease in variance that is statistically significant $(\mathrm{p}<0.10)$ from a) RCP26Historical b) RCP45-Historical, c) RCP60-Historical, and d) RCP85-Historical. 

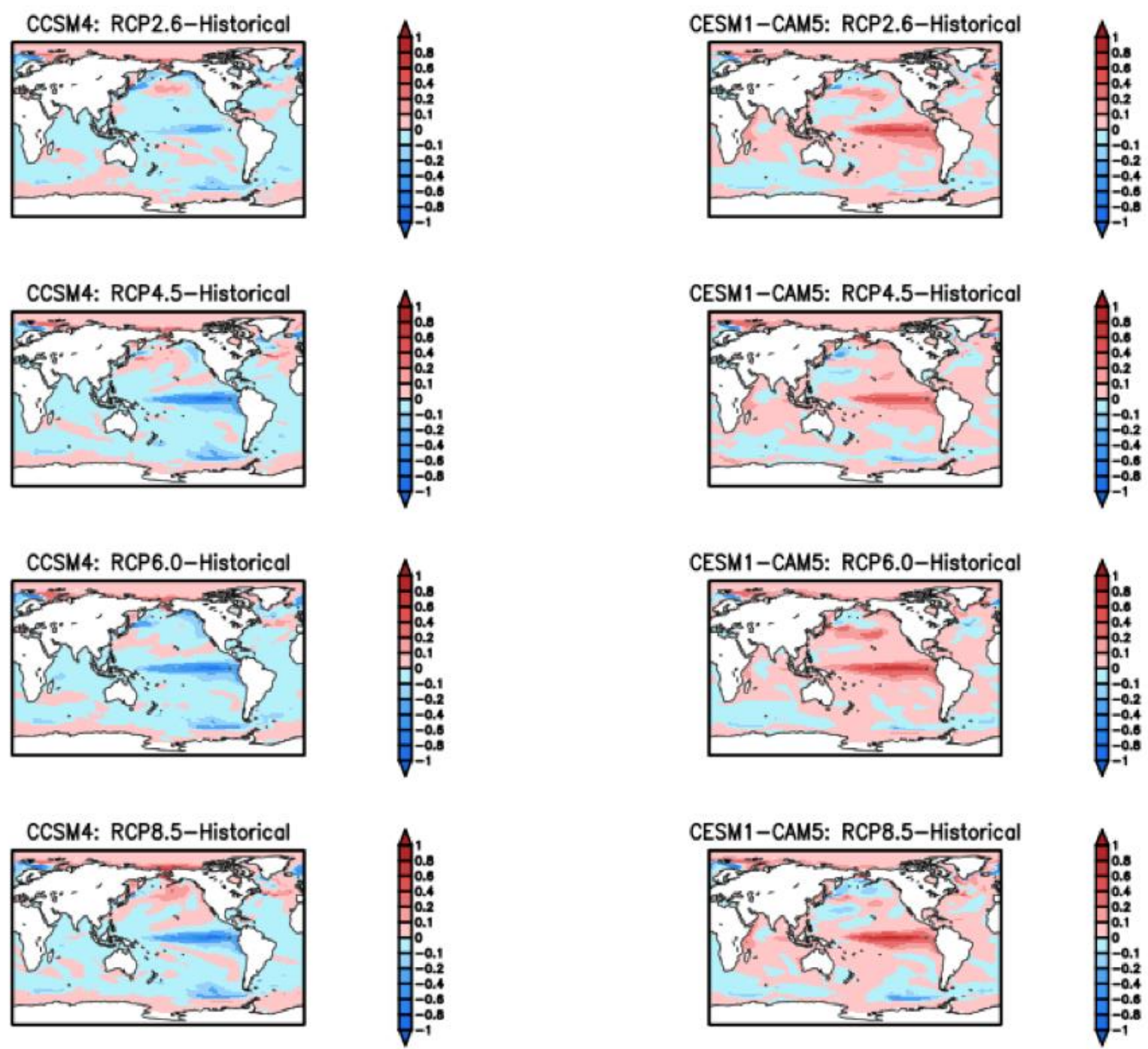

Figure 4.28: Variance difference for CCSM4 on the left column for a) RCP26-Historical c) RCP45-Historical, e) RCP60-Historical, and g) RCP85-Historical and CESM1-CAM5 on the right column for b) RCP26-Historical d) RCP45-Historical, f) RCP60-Historical, and h) RCP85-Historical. Blue indicates decreases in variance and Red denotes increases in variance. 


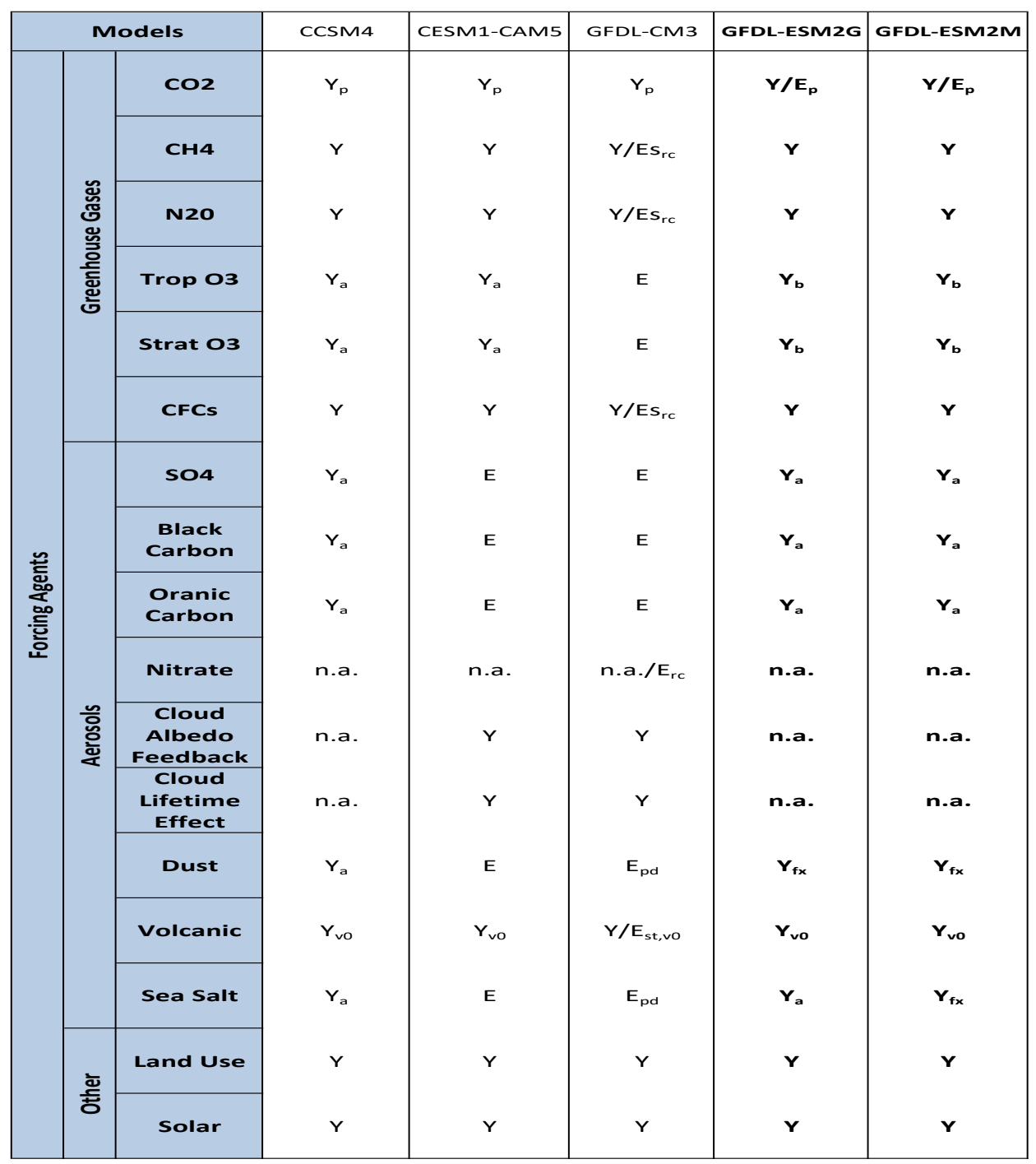

Table 4.1: Radiative forcing agents in CCM4, CESM1-CAM5, GFDL-CM3, GFDLESM2G, and ESM2M (adapted from IPCC, 2013). Earth System Models (ESMs) are highlighted in bold. Entries mean: n.a.: Forcing agent not included in either the Historical or future scenario experiments; Y: Forcing agent included via prescribed concentrations, distributions or time series data; E: Concentrations of forcing agent calculated interactively driven by prescribed emissions; Es: Concentrations of forcing agent calculated interactively constrained by prescribed surface concentrations. Variations in forcing implementations are denoted by subscripts. Subscripts mean: a: 3D distributions specified as monthly 10 year mean concentrations; b: Ozone prescribed using original or slightly modified dataset; p: Physiological forcing effect of $\mathrm{CO} 2$ via plant stomatal response and evapotranspiration included; rc: Separate entries denote different treatments used for radiation and chemistry; fx: Fixed prescribed climatology of dust/seas salt aerosol concentrations with no year-to-year variability; st: Separate entries denote stratosphere and troposphere; v0: Explosive volcanic aerosol returns rapidly in future to zero (or near-zero) background like that in the PiControl experiment 

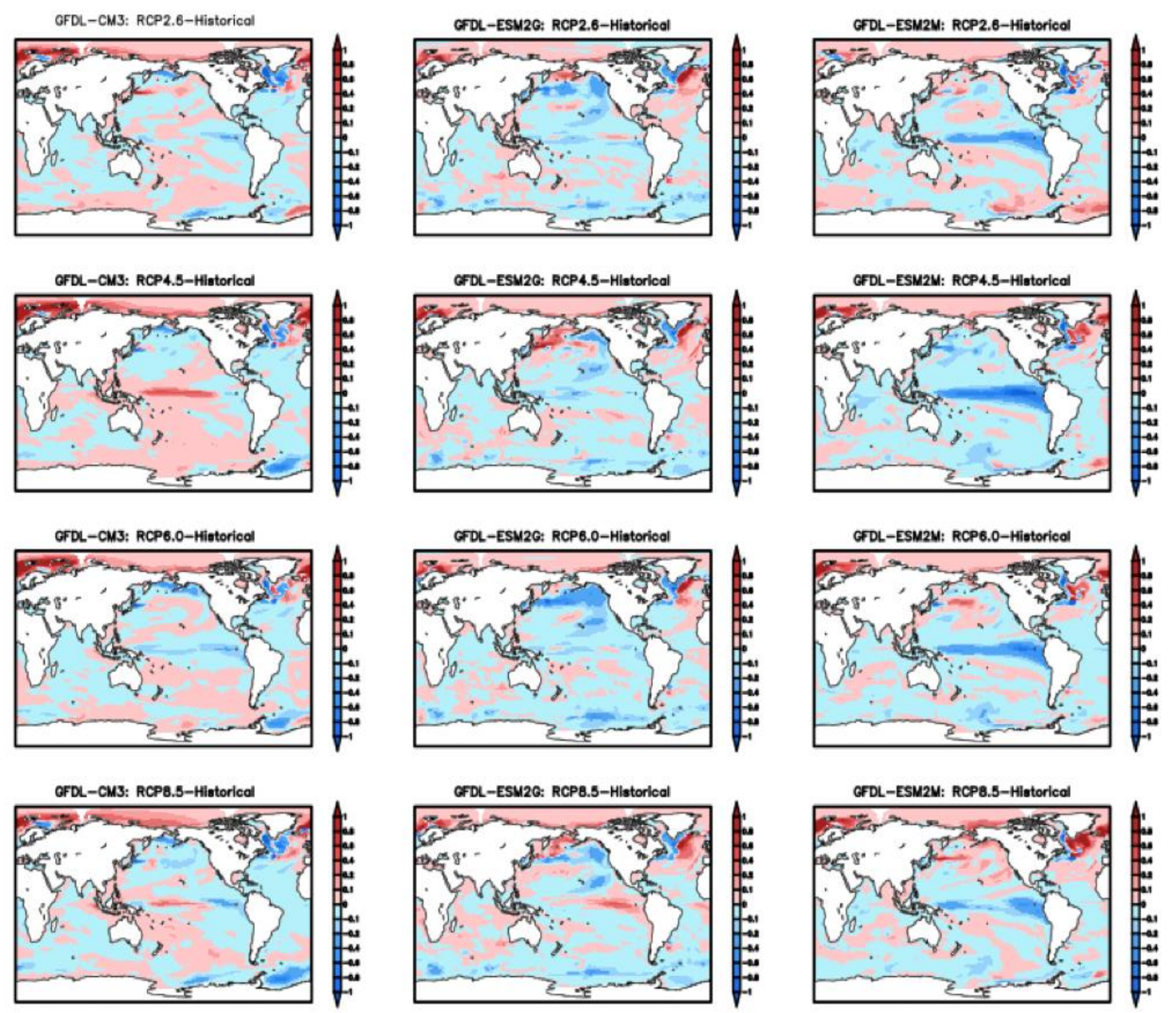

Figure 4.29: Variance difference for GFDL-CM3 is on the left column for a) RCP26Historical d) RCP45-Historical, g) RCP60-Historical, and j) RCP85-Historical. The middle column is the variance difference for GFDL-ESM2G for b) RCP26-Historical, e) RCP45-Historical, h) RCP60-Historical, and k) RCP85-Historical and GFDL-ESM2M on the right column for c) RCP26-Historical f) RCP45-Historical, i) RCP60-Historical, and 1) RCP85-Historical. Blue indicates decreases in variance and Red denotes increases in variance. 


\section{REFERENCES}

Abram, Nerilie J., Michael K. Gagan, Zhengyu Liu, Wahyoe S. Hantoro, Malcolm T. McCulloch, and Bambang W. Suwargadi. "Seasonal characteristics of the Indian Ocean Dipole during the Holocene epoch." Nature 445, no. 7125 (2007): 299-302.

Allen, Robert J., Joel R. Norris, and Mahesh Kovilakam. "Influence of anthropogenic aerosols and the Pacific Decadal Oscillation on tropical belt width." Nature Geoscience 7, no. 4 (2014): ngeo2091.

Ashok, Karumuri, Zhaoyong Guan, and Toshio Yamagata. "Impact of the Indian Ocean dipole on the relationship between the Indian monsoon rainfall and ENSO." Geophysical Research Letters 28, no. 23 (2001): 4499-4502.

Ashok, Karumuri, Guan Zhaoyong, and Toshio Yamagata. "A look at the relationship between the ENSO and the Indian Ocean dipole." Journal of the Meteorological Society of Japan. Ser. II 81, no. 1 (2003): 41-56.

Ashok, Karumuri, Zhaoyong Guan, N. H. Saji, and Toshio Yamagata. "Individual and combined influences of ENSO and the Indian Ocean dipole on the Indian summer monsoon." Journal of Climate 17, no. 16 (2004): 3141-3155.

Balmaseda, Magdalena A., Kevin E. Trenberth, and Erland Källén. "Distinctive climate signals in reanalysis of global ocean heat content." Geophysical Research Letters 40, no. 9 (2013): 1754-1759.

Barbosa, Susana M., and Ole B. Andersen. "Trend patterns in global sea surface temperature." International Journal of Climatology 29, no. 14 (2009): 2049-2055.

Barnett, T. P., B. D. Santer, P. D. Jones, R. S. Bradley, and K. R. Briffa. "Estimates of low frequency natural variability in near-surface air temperature." The Holocene 6, no. 3 (1996): 255-263.

Battisti, David S., and Anthony C. Hirst. "Interannual variability in a tropical atmosphere-ocean model: Influence of the basic state, ocean geometry and nonlinearity." Journal of the atmospheric sciences 46, no. 12 (1989): 1687-1712.

Behera, S. K., R. Krishnan, and T. Yamagata. "Unusual ocean-atmosphere conditions in the tropical Indian Ocean during 1994." Geophysical Research Letters 26, no. 19 (1999): 3001-3004.

Behera, Swadhin K., and Toshio Yamagata. "Subtropical SST dipole events in the southern Indian Ocean." Geophysical Research Letters 28, no. 2 (2001): 327-330. 
Betts, Alan K., and W. Ridgway. "Climatic equilibrium of the atmospheric convective boundary layer over a tropical ocean." Journal of the Atmospheric Sciences 46, no. 17 (1989): 2621-2641.

Biondi, Franco, Alexander Gershunov, and Daniel R. Cayan. "North Pacific decadal climate variability since 1661." Journal of Climate 14, no. 1 (2001): 5-10.

Bjerknes, J_. "1969: Atmospheric teleconnections from the equatorial Pacific." Monthly Weather Review 97: 163-172.

Björnsson, H., and S. A. Venegas. "A manual for EOF and SVD analyses of climatic data." CCGCR Report 97, no. 1 (1997): 112-134.

Box and whisker plots for local climate datasets: Interpretation and creation using Excel 2007/2010. US Department of Commerce, National Oceanic and Atmospheric Administration, National Weather Service, 2011.

Burgman, R. J., Amy C. Clement, C. M. Mitas, J. Chen, and K. Esslinger. "Evidence for atmospheric variability over the Pacific on decadal timescales." Geophysical Research Letters 35, no. 1 (2008).

Burgman, Robert J., Paul S. Schopf, and Ben P. Kirtman. "Decadal modulation of ENSO in a hybrid coupled model." Journal of Climate 21, no. 21 (2008): 5482-5500.

Burgman, Robert, Richard Seager, Amy Clement, and Celine Herweijer. "Role of tropical Pacific SSTs in global medieval hydroclimate: A modeling study." Geophysical Research Letters 37, no. 6 (2010).

Burgman, Robert J., and Youkyoung Jang. "Simulated US Drought Response to Interannual and Decadal Pacific SST Variability." Journal of Climate 28, no. 12 (2015): 4688-4705.

Cai, Wenju, Xiao-Tong Zheng, Evan Weller, Mat Collins, Tim Cowan, Matthieu Lengaigne, Weidong Yu, and Toshio Yamagata. "Projected response of the Indian Ocean Dipole to greenhouse warming." Nature geoscience 6, no. 12 (2013): 999-1007.

Cane, Mark A., Amy C. Clement, Alexey Kaplan, Yochanan Kushnir, Dmitri Pozdnyakov, Richard Seager, Stephen E. Zebiak, and Ragu Murtugudde. "Twentiethcentury sea surface temperature trends." Science 275, no. 5302 (1997): 957-960.

Carton, James A., and Bohua Huang. "Warm events in the tropical Atlantic." Journal of Physical Oceanography 24, no. 5 (1994): 888-903. 
Carton, James A., Xianhe Cao, Benjamin S. Giese, and Arlindo M. Da Silva. "Decadal and interannual SST variability in the tropical Atlantic Ocean." Journal of Physical Oceanography 26, no. 7 (1996): 1165-1175.

Cavalieri, D. J., and C. L. Parkinson. "Antarctic sea ice variability and trends, 19792006." Journal of Geophysical Research: Oceans 113, no. C7 (2008).

Chang, Ping, Link Ji, and Hong Li. "A decadal climate variation in the tropical Atlantic Ocean from thermodynamic air-sea interactions." Nature 385, no. 6616 (1997): 516.

Chen, Xianyao, and Ka-Kit Tung. "Varying planetary heat sink led to global-warming slowdown and acceleration." Science345, no. 6199 (2014): 897-903.

Chiang, John CH, Yochanan Kushnir, and Alessandra Giannini. "Deconstructing Atlantic Intertropical Convergence Zone variability: Influence of the local cross-equatorial sea surface temperature gradient and remote forcing from the eastern equatorial Pacific." Journal of Geophysical Research: Atmospheres 107, no. D1 (2002).

Chiang, John CH, and Daniel J. Vimont. "Analogous Pacific and Atlantic meridional modes of tropical atmosphere-ocean variability." Journal of Climate 17, no. 21 (2004): 4143-4158.

Chu, Jung-Eun, Kyung-Ja Ha, June-Yi Lee, Bin Wang, Byeong-Hee Kim, and Chul Eddy Chung. "Future change of the Indian Ocean basin-wide and dipole modes in the CMIP5." Climate dynamics 43, no. 1-2 (2014): 535-551.

Clement, Amy C., Richard Seager, Mark A. Cane, and Stephen E. Zebiak. "An ocean dynamical thermostat." Journal of Climate 9, no. 9 (1996): 2190-2196.

Clement, Amy C., Robert Burgman, and Joel R. Norris. "Observational and model evidence for positive low-level cloud feedback." Science 325, no. 5939 (2009): 460-464.

Collins, Matthew. "El Niño-or La Niña-like climate change?." Climate Dynamics 24, no. 1 (2005): 89-104.

Comrie, Andrew C., and Gregory J. McCabe. "Global air temperature variability independent of sea-surface temperature influences." Progress in Physical Geography 37, no. 1 (2013): 29-35.

Comrie, Andrew C., and Gregory J. McCabe. "Global air temperature variability independent of sea-surface temperature influences." Progress in Physical Geography 37, no. 1 (2013): 29-35.

Comiso, Josefino C., Claire L. Parkinson, Robert Gersten, and Larry Stock. "Accelerated decline in the Arctic sea ice cover." Geophysical research letters 35, no. 1 (2008). 
Comiso, Josefino C., and Fumihiko Nishio. "Trends in the sea ice cover using enhanced and compatible AMSR-E, SSM/I, and SMMR data." Journal of Geophysical Research: Oceans 113, no. C2 (2008).

D'Arrigo, Rosanne, Gregory Wiles, Gordon Jacoby, and Ricardo Villalba. "North Pacific sea surface temperatures: past variations inferred from tree rings." Geophysical Research Letters 26, no. 17 (1999): 2757-2760.

Delworth, T., S. Manabe, and R. J. Stouffer. "Interdecadal variations of the thermohaline circulation in a coupled ocean-atmosphere model." Journal of Climate 6, no. 11 (1993): 1993-2011.

Delworth, Thomas L., and Michael E. Mann. "Observed and simulated multidecadal variability in the Northern Hemisphere." Climate Dynamics 16, no. 9 (2000): 661-676.

Deser, Clara, John E. Walsh, and Michael S. Timlin. "Arctic sea ice variability in the context of recent atmospheric circulation trends." Journal of Climate 13, no. 3 (2000): 617-633.

Deser, Clara, Michael A. Alexander, Shang-Ping Xie, and Adam S. Phillips. "Sea surface temperature variability: Patterns and mechanisms." Annual Review of Marine Science 2 (2010): 115-143.

Deser, Clara, Robert Tomas, Michael Alexander, and David Lawrence. "The seasonal atmospheric response to projected Arctic sea ice loss in the late twenty-first century." Journal of Climate 23, no. 2 (2010): 333-351.

Deser, Clara, Reto Knutti, Susan Solomon, and Adam S. Phillips. "Communication of the role of natural variability in future North American climate." Nature Climate Change 2, no. 11 (2012): 775-779.

Deser, Clara, Adam Phillips, Vincent Bourdette, and Haiyan Teng. "Uncertainty in climate change projections: the role of internal variability." Climate dynamics 38, no. 3-4 (2012): 527-546.

DiNezio, Pedro N., Amy C. Clement, Gabriel A. Vecchi, Brian J. Soden, Benjamin P. Kirtman, and Sang-Ki Lee. "Climate response of the equatorial Pacific to global warming." Journal of Climate 22, no. 18 (2009): 4873-4892.

DiNezio, Pedro, Amy Clement, and Gabriel Vecchi. "Reconciling differing views of tropical Pacific climate change." Eos, Transactions American Geophysical Union 91, no. 16 (2010): 141-142. 
Dommenget, Dietmar, and Mojib Latif. "Interannual to decadal variability in the tropical Atlantic." Journal of Climate 13, no. 4 (2000): 777-792.

Dommenget, Dietmar, and Mojib Latif. "Generation of hyper climate modes." Geophysical Research Letters 35, no. 2 (2008).

Drijfhout, S. S., A. T. Blaker, S. A. Josey, A. J. G. Nurser, B. Sinha, and M. A. Balmaseda. "Surface warming hiatus caused by increased heat uptake across multiple ocean basins." Geophysical Research Letters 41, no. 22 (2014): 7868-7874.

Du, Yan, Shang-Ping Xie, Gang Huang, and Kaiming Hu. "Role of air-sea interaction in the long persistence of El Niño-induced north Indian Ocean warming." Journal of Climate 22, no. 8 (2009): 2023-2038.

Dunne, John P., Jasmin G. John, Alistair J. Adcroft, Stephen M. Griffies, Robert W. Hallberg, Elena Shevliakova, Ronald J. Stouffer et al. "GFDL's ESM2 global coupled climate-carbon earth system models. Part I: Physical formulation and baseline simulation characteristics." Journal of Climate 25, no. 19 (2012): 6646-6665.

Easterling, David R., and Michael F. Wehner. "Is the climate warming or cooling?." Geophysical Research Letters 36, no. 8 (2009).

Enfield, David B., and Dennis A. Mayer. "Tropical Atlantic sea surface temperature variability and its relation to El Niño-Southern Oscillation." Journal of Geophysical Research: Oceans 102, no. C1 (1997): 929-945.

Enfield, David B., and Alberto M. Mestas-Nuñez. "Multiscale variabilities in global sea surface temperatures and their relationships with tropospheric climate patterns." Journal of Climate 12, no. 9 (1999): 2719-2733.

Enfield, DAVID B., and ALBERTO M. Mestas-Nuñez. Global modes of ENSO and nonENSO sea surface temperature variability and their associations with climate. Cambridge University Press, 2000.

Enfield, David B., Alberto M. Mestas-Nuñez, and Paul J. Trimble. "The Atlantic multidecadal oscillation and its relation to rainfall and river flows in the continental US." Geophysical Research Letters 28, no. 10 (2001): 2077-2080.

England, Matthew H., Shayne McGregor, Paul Spence, Gerald A. Meehl, Axel Timmermann, Wenju Cai, Alex Sen Gupta, Michael J. McPhaden, Ariaan Purich, and Agus Santoso. "Recent intensification of wind-driven circulation in the Pacific and the ongoing warming hiatus." Nature Climate Change 4, no. 3 (2014): 222-227. 
Estrada, Francisco, and Pierre Perron. "Extracting and analyzing the warming trend in global and hemispheric temperatures." Journal of Time Series Analysis 38, no. 5 (2017): 711-732.

Folland, C. K., T. N. Palmer, and D. E. Parker. "Sahel rainfall and worldwide sea temperatures, 1901-85." Nature 320, no. 6063 (1986): 602-607.

Foster, Grant, and Stefan Rahmstorf. "Global temperature evolution 1979? 2010." Environmental Research Letters 6, no. 4 (2011): 044022.

Franzke, Christian. "Nonlinear trends, long-range dependence, and climate noise properties of surface temperature." Journal of Climate 25, no. 12 (2012): 4172-4183.

Furtado, Jason C., Emanuele Di Lorenzo, Niklas Schneider, and Nicholas A. Bond. "North Pacific decadal variability and climate change in the IPCC AR4 models." Journal of Climate 24, no. 12 (2011): 3049-3067.

Fyfe, John C., Nathan P. Gillett, and Francis W. Zwiers. "Overestimated global warming over the past 20 years." Nature Climate Change 3, no. 9 (2013): 767-769.

Garreaud, RenéD, and David S. Battisti. "Interannual (ENSO) and interdecadal (ENSOlike) variability in the Southern Hemisphere tropospheric circulation*." Journal of Climate 12, no. 7 (1999): 2113-2123.

Gent, Peter R., Gokhan Danabasoglu, Leo J. Donner, Marika M. Holland, Elizabeth C. Hunke, Steve R. Jayne, David M. Lawrence et al. "The community climate system model version 4." Journal of Climate 24, no. 19 (2011): 4973-4991.

Ghil, Michael. "Natural climate variability." Encyclopedia of global environmental change 1 (2002): 544-549.

Giannini, A., R. Saravanan, and P. Chang. "The preconditioning role of tropical Atlantic variability in the development of the ENSO teleconnection: implications for the prediction of Nordeste rainfall." Climate Dynamics 22, no. 8 (2004): 839-855.

Goldenberg, Stanley B., Christopher W. Landsea, Alberto M. Mestas-Nuñez, and William M. Gray. "The recent increase in Atlantic hurricane activity: Causes and implications." Science 293, no. 5529 (2001): 474-479.

Gornitz, Vivien, Sergej Lebedeff, and James Hansen. "Global sea level trend in the past century." Science 215, no. 4540 (1982): 1611-1614.

Grossmann, Iris, and Philip J. Klotzbach. "A review of North Atlantic modes of natural variability and their driving mechanisms." Journal of Geophysical Research: Atmospheres 114, no. D24 (2009). 
Gu, Daifang, and S. GH Philander. "Interdecadal climate fluctuations that depend on exchanges between the tropics and extratropics." Science 275, no. 5301 (1997): 805-807.

Guemas, Virginie, Francisco J. Doblas-Reyes, Isabel Andreu-Burillo, and Muhammad Asif. "Retrospective prediction of the global warming slowdown in the past decade." Nature Climate Change 3, no. 7 (2013): 649-653.

Guilyardi, Eric, Hugo Bellenger, Mat Collins, Samantha Ferrett, Wenju Cai, and Andrew Wittenberg. "A first look at ENSO in CMIP5." Clivar Exchanges 17, no. 1 (2012): 29-32.

Hagedorn, Renate, FRANCISCO J. DOBLAS-REYES, and T. N. Palmer. "The rationale behind the success of multi-model ensembles in seasonal forecasting-I. Basic concept." Tellus A57, no. 3 (2005): 219-233.

Han, Weiqing, Jérôme Vialard, Michael J. McPhaden, Tong Lee, Yukio Masumoto, Ming Feng, and Will PM De Ruijter. "Indian Ocean decadal variability: A review." Bulletin of the American Meteorological Society 95, no. 11 (2014): 1679-1703.

Hannachi, A. "A Primer for EOF Analysis of Climate Data. Department of Meteorology, University of Reading." Reading RG6 6BB, UK (2004).

Hannachi, A., I. T. Jolliffe, and D. B. Stephenson. "Empirical orthogonal functions and related techniques in atmospheric science: A review." International journal of climatology 27, no. 9 (2007): 1119-1152.

Hasselmann, Klaus. "Stochastic climate models part I. Theory." tellus 28, no. 6 (1976): 473-485.

Hawkins, Ed, and Rowan Sutton. "The potential to narrow uncertainty in regional climate predictions." Bulletin of the American Meteorological Society 90, no. 8 (2009): 10951107.

Hawkins, E., and R. Sutton. "Time of emergence of climate signals." Geophysical Research Letters 39, no. 1 (2012).

Held, Isaac M., and Brian J. Soden. "Robust responses of the hydrological cycle to global warming." Journal of Climate 19, no. 21 (2006): 5686-5699.

Hetzinger, Steffen, J. Halfar, J. V. Mecking, Noel S. Keenlyside, A. Kronz, R. S. Steneck, W. H. Adey, and P. A. Lebednik. "Marine proxy evidence linking decadal North Pacific and Atlantic climate." Climate Dynamics 39, no. 6 (2012): 1447-1455.

Holland, Paul R., and Ron Kwok. "Wind-driven trends in Antarctic sea-ice drift." Nature Geoscience 5, no. 12 (2012): 872. 
Hsu, Pang-chi, Tim Li, Jing-Jia Luo, Hiroyuki Murakami, Akio Kitoh, and Ming Zhao. "Increase of global monsoon area and precipitation under global warming: A robust signal?." Geophysical Research Letters 39, no. 6 (2012).

Hsu, Pang-chi, Tim Li, Hiroyuki Murakami, and Akio Kitoh. "Future change of the global monsoon revealed from 19 CMIP5 models." Journal of Geophysical Research: Atmospheres 118, no. 3 (2013): 1247-1260.

$\mathrm{Hu}$, Y., and Q. Fu. "Observed poleward expansion of the Hadley circulation since 1979." Atmospheric Chemistry and Physics 7, no. 19 (2007): 5229-5236.

Huang, Norden E., Zheng Shen, Steven R. Long, Manli C. Wu, Hsing H. Shih, Quanan Zheng, Nai-Chyuan Yen, Chi Chao Tung, and Henry H. Liu. "The empirical mode decomposition and the Hilbert spectrum for nonlinear and non-stationary time series analysis." In Proceedings of the Royal Society of London A: mathematical, physical and engineering sciences, vol. 454, no. 1971, pp. 903-995. The Royal Society, 1998.

Huang, Bohua, and James L. Kinter. "Interannual variability in the tropical Indian Ocean." Journal of Geophysical Research: Oceans 107, no. C11 (2002).

Huang, Bohua, Paul S. Schopf, and Ziqin Pan. "The ENSO effect on the tropical Atlantic variability: A regionally coupled model study." Geophysical Research Letters 29, no. 21 (2002).

Huang, Boyin, Viva F. Banzon, Eric Freeman, Jay Lawrimore, Wei Liu, Thomas C. Peterson, Thomas M. Smith, Peter W. Thorne, Scott D. Woodruff, and Huai-Min Zhang. "Extended reconstructed sea surface temperature version 4 (ERSST. v4). Part I: upgrades and intercomparisons." Journal of Climate 28, no. 3 (2015): 911-930.

IPCC, 2007: Climate Change 2007: The Physical Science Basis. Contribution of Working Group I to the Fourth Assessment Report of the Intergovernmental Panel on Climate Change [Solomon, S., D. Qin, M. Manning, Z. Chen, M. Marquis, K.B. Averyt, M. Tignor and H.L. Miller (eds.)]. Cambridge University Press, Cambridge, United Kingdom and New York, NY, USA, 996 pp.

IPCC, 2013: Climate Change 2013: The Physical Science Basis. Contribution of Working Group I to the Fifth Assessment Report of the Intergovern- mental Panel on Climate Change [Stocker, T.F., D. Qin, G.-K. Plattner, M. Tignor, S.K. Allen, J. Boschung, A. Nauels, Y. Xia, V. Bex and P.M. Midgley (eds.)]. Cambridge University Press, Cambridge, United Kingdom and New York, NY, USA, 1535 pp.

Jin, Fei-Fei. "Low-Frequency Modes of Tropical Ocean Dynamics*." Journal of Climate 14, no. 18 (2001): 3874-3881. 
Johannessen, Ola M., Lennart Bengtsson, Martin W. Miles, Svetlana I. Kuzmina, Vladimir A. Semenov, Genrikh V. Alekseev, Andrei P. Nagurnyi et al. "Arctic climate change: Observed and modelled temperature and sea-ice variability." Tellus A 56, no. 4 (2004): 328-341.

Johanson, Celeste M., and Qiang Fu. "Hadley cell widening: Model simulations versus observations." Journal of Climate22, no. 10 (2009): 2713-2725.

Kaufmann, Robert K., Heikki Kauppi, and James H. Stock. "Does temperature contain a stochastic trend? Evaluating conflicting statistical results." Climatic change 101, no. 3-4 (2010): 395-405.

Kaufmann, Robert K., Heikki Kauppi, Michael L. Mann, and James H. Stock. "Reconciling anthropogenic climate change with observed temperature 1998-2008." Proceedings of the National Academy of Sciences 108, no. 29 (2011): 11790-11793.

Kaufmann, Robert K., Heikki Kauppi, Michael L. Mann, and James H. Stock. "Does temperature contain a stochastic trend: linking statistical results to physical mechanisms." Climatic change 118, no. 3-4 (2013): 729-743.

Keenlyside, Noel S., and Mojib Latif. "Understanding equatorial Atlantic interannual variability." Journal of climate 20, no. 1 (2007): 131-142.

Kennedy J.J., Rayner, N.A., Smith, R.O., Saunby, M. and Parker, D.E. (2011b). Reassessing biases and other uncertainties in sea-surface temperature observations since 1850 part 1: measurement and sampling errors. J. Geophys. Res., 116, D14103, doi:10.1029/2010JD015218

Kerr, Richard A. "A North Atlantic climate pacemaker for the centuries." Science 288, no. 5473 (2000): 1984-1985.

Kilbourne, K. H., T. M. Quinn, R. Webb, T. Guilderson, J. Nyberg, and A. Winter. "Paleoclimate proxy perspective on Caribbean climate since the year 1751: Evidence of cooler temperatures and multidecadal variability." Paleoceanography 23, no. 3 (2008).

Kim, Seon Tae, and Jin-Yi Yu. "The two types of ENSO in CMIP5 models." Geophysical Research Letters 39, no. 11 (2012).

Kirtman, Ben P. "Oceanic Rossby wave dynamics and the ENSO period in a coupled model." Journal of climate 10, no. 7 (1997): 1690-1704.

Kirtman, Ben P., and Paul S. Schopf. "Decadal variability in ENSO predictability and prediction." Journal of Climate 11, no. 11 (1998): 2804-2822. 
Kirtman, Ben P., and J. Shukla. "Interactive coupled ensemble: A new coupling strategy for CGCMs." Geophysical research letters 29, no. 10 (2002).

Kirtman, Ben P., Cecilia Bitz, Frank Bryan, William Collins, John Dennis, Nathan Hearn, James L. Kinter III et al. "Impact of ocean model resolution on CCSM climate simulations." Climate dynamics 39, no. 6 (2012): 1303-1328.

Kitoh, Akio, Hirokazu Endo, K. Krishna Kumar, Iracema FA Cavalcanti, Prashant Goswami, and Tianjun Zhou. "Monsoons in a changing world: a regional perspective in a global context." Journal of Geophysical Research: Atmospheres 118, no. 8 (2013): 30533065.

Kleeman, Richard, Julian P. McCreary, and Barry A. Klinger. "A mechanism for generating ENSO decadal variability." Geophysical Research Letters 26, no. 12 (1999): 1743-1746.

Klein, Stephen A., Brian J. Soden, and Ngar-Cheung Lau. "Remote sea surface temperature variations during ENSO: Evidence for a tropical atmospheric bridge." Journal of Climate 12, no. 4 (1999): 917-932.

Knight, Jeff R., Robert J. Allan, Chris K. Folland, Michael Vellinga, and Michael E. Mann. "A signature of persistent natural thermohaline circulation cycles in observed climate." Geophysical Research Letters 32, no. 20 (2005).

Knight, Jeff R., Chris K. Folland, and Adam A. Scaife. "Climate impacts of the Atlantic multidecadal oscillation." Geophysical Research Letters 33, no. 17 (2006).

Knutson, Thomas R., and Syukuro Manabe. "Time-mean response over the tropical Pacific to increased C02 in a coupled ocean-atmosphere model." Journal of Climate 8, no. 9 (1995): 2181-2199.

Knutson, Thomas R., and Syukuro Manabe. "Model assessment of decadal variability and trends in the tropical Pacific Ocean." Journal of Climate 11, no. 9 (1998): 2273-2296.

Kosaka, Yu, and Shang-Ping Xie. "Recent global-warming hiatus tied to equatorial Pacific surface cooling." Nature 501, no. 7467 (2013): 403-407.

Krishnamurti, T. N., C. M. Kishtawal, Timothy E. LaRow, David R. Bachiochi, Zhan Zhang, C. Eric Williford, Sulochana Gadgil, and Sajani Surendran. "Improved weather and seasonal climate forecasts from multimodel superensemble." Science285, no. 5433 (1999): 1548-1550.

Krishnamurthy, Lakshmi, and V. Krishnamurthy. "Decadal and interannual variability of the Indian Ocean SST." Climate Dynamics 46, no. 1-2 (2016): 57-70. 
Kushnir, Yochanan. "Interdecadal variations in North Atlantic sea surface temperature and associated atmospheric conditions." Journal of Climate 7, no. 1 (1994): 141-157.

Mahlstein, I., R. Knutti, S. Solomon, and R. W. Portmann. "Early onset of significant local warming in low latitude countries." Environmental Research Letters 6, no. 3 (2011): 034009.

Lai, Kon S., and Mann Yoon. "Nonlinear trend stationarity in global and hemispheric temperatures." Applied Economics Letters 25, no. 1 (2018): 15-18.

Latif, Mojib, and Timothy P. Barnett. "Interactions of the tropical oceans." Journal of Climate 8, no. 4 (1995): 952-964.

Lawrence, S. P., D. T. Llewellyn-Jones, and S. J. Smith. "The measurement of climate change using data from the Advanced Very High Resolution and Along Track Scanning Radiometers." Journal of Geophysical Research: Oceans 109, no. C8 (2004).

Lennartz, S., and A. Bunde. "Trend evaluation in records with long-term memory: Application to global warming." Geophysical Research Letters 36, no. 16 (2009).

Li, Tony W., and Noel C. Baker. "Detecting Warming Hiatus Periods in CMIP5 Climate Model Projections." International Journal of Atmospheric Sciences 2016 (2016).

Liu, Jiping, Judith A. Curry, Huijun Wang, Mirong Song, and Radley M. Horton. "Impact of declining Arctic sea ice on winter snowfall." Proceedings of the National Academy of Sciences 109, no. 11 (2012): 4074-4079.

Lu, Jian, Gabriel A. Vecchi, and Thomas Reichler. "Expansion of the Hadley cell under global warming." Geophysical Research Letters 34, no. 6 (2007).

Lu, Jian, Clara Deser, and Thomas Reichler. "Cause of the widening of the tropical belt since 1958." Geophysical Research Letters 36, no. 3 (2009).

Mahlstein, I., R. Knutti, S. Solomon, and R. W. Portmann. "Early onset of significant local warming in low latitude countries." Environmental Research Letters 6, no. 3 (2011): 034009.

Mahlstein, Irina, Peter R. Gent, and Susan Solomon. "Historical Antarctic mean sea ice area, sea ice trends, and winds in CMIP5 simulations." Journal of Geophysical Research: Atmospheres 118, no. 11 (2013): 5105-5110.

Mantua, Nathan J., Steven R. Hare, Yuan Zhang, John M. Wallace, and Robert C. Francis. "A Pacific interdecadal climate oscillation with impacts on salmon production." Bulletin of the american Meteorological Society 78, no. 6 (1997): 1069-1079. 
Mantua, Nathan J., and Steven R. Hare. "The Pacific decadal oscillation." Journal of oceanography 58, no. 1 (2002): 35-44.

Marshall, John, Yochanan Kushnir, David Battisti, Ping Chang, Arnaud Czaja, Robert Dickson, James Hurrell, M. I. C. H. A. E. L. McCARTNEY, R. Saravanan, and Martin Visbeck. "North Atlantic climate variability: phenomena, impacts and mechanisms." International Journal of Climatology21, no. 15 (2001): 1863-1898.

Masui, Toshihiko, Kenichi Matsumoto, Yasuaki Hijioka, Tsuguki Kinoshita, Toru Nozawa, Sawako Ishiwatari, Etsushi Kato, P. R. Shukla, Yoshiki Yamagata, and Mikiko Kainuma. "An emission pathway for stabilization at $6 \mathrm{Wm}-2$ radiative forcing." Climatic change 109, no. 1-2 (2011): 59.

McCabe, Gregory J., Michael A. Palecki, and Julio L. Betancourt. "Pacific and Atlantic Ocean influences on multidecadal drought frequency in the United States." Proceedings of the National Academy of Sciences 101, no. 12 (2004): 4136-4141.

McCormick, M. Patrick, Larry W. Thomason, and Charles R. Trepte. "Atmospheric effects of the Mt Pinatubo eruption." Nature 373, no. 6513 (1995): 399.

Meehl, Gerald A., and Warren M. Washington. "El Niño-like climate change in a model with increased atmospheric CO2 concentrations." Nature 382, no. 6586 (1996): 56.

Meehl, Gerald A., Julie M. Arblaster, John T. Fasullo, Aixue Hu, and Kevin E. Trenberth. "Model-based evidence of deep-ocean heat uptake during surface-temperature hiatus periods." Nature Climate Change 1, no. 7 (2011): 360-364.

Meehl, Gerald A., Warren M. Washington, Julie M. Arblaster, Aixue Hu, Haiyan Teng, Claudia Tebaldi, Benjamin N. Sanderson et al. "Climate system response to external forcings and climate change projections in CCSM4." Journal of Climate 25, no. 11 (2012): 3661-3683.

Meehl, G. A., Washington, W. M., Arblaster, J. M., Hu, A., Teng, H., Kay, J. E., ... \& Strand, W. G. (2013). Climate change projections in CESM1 (CAM5) compared to CCSM4. Journal of Climate, 26(17), 6287-6308.

Merryfield, William J. "Changes to ENSO under $\mathrm{CO} 2$ doubling in a multimodel ensemble." Journal of Climate 19, no. 16 (2006): 4009-4027.

Meyers, S. D., J. J. O'Brien, and E. Thelin. "Reconstruction of Monthly SST in the Tropical Pacific Ocean during 1868-1993Using Adaptive Climate Basis Functions." Monthly Weather Review 127, no. 7 (1999): 1599-1612. 
Minnis, P., E. F. Harrison, L. L. Stowe, G. G. Gibson, F. M. Denn, D. R. Doelling, and W. L. Smith. "Radiative climate forcing by the Mount Pinatubo eruption." Science 259, no. 5100 (1993): 1411-1415.

Minobe, Shoshiro, Niklas Schneider, Clara Deser, Zhengyu Liu, Nathan Mantua, Hisashi Nakamura, and Masami Nonaka. "Pacific Decadal Variability: A Review." In First International CLIVAR Conference, Baltimore, MD, USA. 2004.

Mitas, Christos M., and Amy Clement. "Has the Hadley cell been strengthening in recent decades?." Geophysical Research Letters 32, no. 3 (2005).

Morice, Colin P., John J. Kennedy, Nick A. Rayner, and Phil D. Jones. "Quantifying uncertainties in global and regional temperature change using an ensemble of observational estimates: The HadCRUT4 data set." Journal of Geophysical Research: Atmospheres (1984-2012) 117, no. D8 (2012).

Morioka, Yushi, Tomoki Tozuka, Sebastien Masson, Pascal Terray, Jing-Jia Luo, and Toshio Yamagata. "Subtropical dipole modes simulated in a coupled general circulation model." Journal of Climate 25, no. 12 (2012): 4029-4047.

Morioka, Yushi, Tomoki Tozuka, and Toshio Yamagata. "How is the Indian Ocean subtropical dipole excited?." Climate dynamics 41, no. 7-8 (2013): 1955-1968.

Moss, Richard H., Jae A. Edmonds, Kathy A. Hibbard, Martin R. Manning, Steven K. Rose, Detlef P. Van Vuuren, Timothy R. Carter et al. "The next generation of scenarios for climate change research and assessment." Nature 463, no. 7282 (2010): 747.

Moura, Antonio D., and Jagadish Shukla. "On the dynamics of droughts in northeast Brazil: Observations, theory and numerical experiments with a general circulation model." Journal of the Atmospheric Sciences 38, no. 12 (1981): 2653-2675.

Neale, Richard B., Chih-Chieh Chen, Andrew Gettelman, Peter H. Lauritzen, Sungsu Park, David L. Williamson, Andrew J. Conley et al. "Description of the NCAR community atmosphere model (CAM 5.0)." NCAR Tech. Note NCAR/TN-486+ STR 1, no. 1 (2010): 1-12.

Neelin, J. David, David S. Battisti, Anthony C. Hirst, Fei-Fei Jin, Yoshinobu Wakata, Toshio Yamagata, and Stephen E. Zebiak. "ENSO theory." Journal of Geophysical Research: Oceans 103, no. C7 (1998): 14261-14290.

NOAA National Centers for Environmental Information, State of the Climate: Global Analysis for Annual 2015, published online January 2016, retrieved on February 3, 2016 from http://www.ncdc.noaa.gov/sotc/global/201513. 
Nobre, Paulo, and J. Shukla. "Variations of sea surface temperature, wind stress, and rainfall over the tropical Atlantic and South America." Journal of climate 9, no. 10 (1996): 2464-2479.

Okumura, Yuko, and Shang-Ping Xie. "Some overlooked features of tropical Atlantic climate leading to a new Niño-like phenomenon." Journal of climate19, no. 22 (2006): 5859-5874.

Østvand, Lene, Kristoffer Rypdal, and Martin Rypdal. "Statistical significance of rising and oscillatory trends in global ocean and land temperature in the past 160 years." arXiv preprint arXiv:1307.6991 (2013).

Overland, James E., Jennifer A. Francis, Edward Hanna, and Muyin Wang. "The recent shift in early summer Arctic atmospheric circulation." Geophysical Research Letters 39, no. 19 (2012).

Palmer, T. N., A. Alessandri, U. Andersen, P. Cantelaube, M. Davey, Pascale Delécluse, Michel Déqué et al. "Development of a European multimodel ensemble system for seasonal-to-interannual prediction (DEMETER)." Bulletin of the American Meteorological Society 85, no. 6 (2004): 853-872.

Palmer, T. N., F. J. Doblas-Reyes, R. Hagedorn, and A. Weisheimer. "Probabilistic prediction of climate using multi-model ensembles: from basics to applications." Philosophical Transactions of the Royal Society of London B: Biological Sciences 360, no. 1463 (2005): 1991-1998.

Park, Wonsun, and Mojib Latif. "Pacific and Atlantic multidecadal variability in the Kiel Climate Model." Geophysical Research Letters 37, no. 24 (2010).

Parker, D. E., H. Wilson, Phil D. Jones, J. R. Christy, and Chris K. Folland. "The impact of Mount Pinatubo on world-wide temperatures." International Journal of Climatology: A Journal of the Royal Meteorological Society 16, no. 5 (1996): 487-497.

Prinsenberg, S. J., I. K. Peterson, S. Narayanan, and J. U. Umoh. "Interaction between atmosphere, ice cover, and ocean off Labrador and Newfoundland from 1962 to 1992." Canadian Journal of Fisheries and Aquatic Sciences 54, no. S1 (1997): 30-39.

Rahmstorf, Stefan. "Shifting seas in the greenhouse?." Nature399, no. 6736 (1999): 523.

Rahmstorf, Stefan, Grant Foster, and Niamh Cahill. "Global temperature evolution: recent trends and some pitfalls." Environmental Research Letters 12, no. 5 (2017): 054001. 
Ramanathan, Vi, and W. Collins. "Thermodynamic regulation of ocean warming by cirrus clouds deduced from observations of the 1987 El Nino." Nature 351, no. 6321 (1991): 27.

Reichler, Thomas, and Junsu Kim. "How well do coupled models simulate today's climate?." Bulletin of the American Meteorological Society 89, no. 3 (2008): 303-311.

Riahi, K., V. Krey, S. Rao, V. Chirkov, G. Fischer, P. Kolp, G. Kindermann, N. Nakicenovic, and P. Rafai. "RCP-8.5: exploring the consequence of high emission trajectories." Climatic Change. doi 10 (2011): 1007.

Roberts, Craig D., Matthew D. Palmer, Doug McNeall, and Matthew Collins. "Quantifying the likelihood of a continued hiatus in global warming." Nature Climate Change 5, no. 4 (2015): 337.

Robock, Alan, and Jianping Mao. "The volcanic signal in surface temperature observations." Journal of Climate 8, no. 5 (1995): 1086-1103.

Rosenlof, Karen H., and George C. Reid. "Trends in the temperature and water vapor content of the tropical lower stratosphere: Sea surface connection." Journal of Geophysical Research: Atmospheres 113, no. D6 (2008).

Ruiz-Barradas, Alfredo, James A. Carton, and Sumant Nigam. "Structure of interannualto-decadal climate variability in the tropical Atlantic sector." Journal of Climate 13, no. 18 (2000): 3285-3297.

Rypdal, Kristoffer. "Attribution in the presence of a long-memory climate response." Earth System Dynamics 6, no. 2 (2015): 719.

Saji, N. H., B. N. Goswami, P. N. Vinayachandran, and T. Yamagata. "A dipole mode in the tropical Indian Ocean." Nature 401, no. 6751 (1999): 360-363.

Saji, N. H., S. P. Xie, and T. Yamagata. "Tropical Indian Ocean variability in the IPCC twentieth-century climate simulations." Journal of Climate 19, no. 17 (2006): 4397-4417.

Santer, Benjamin D., T. M. L. Wigley, J. S. Boyle, Dian J. Gaffen, J. J. Hnilo, D. Nychka, D. E. Parker, and K. E. Taylor. "Statistical significance of trends and trend differences in layer-average atmospheric temperature time series." Journal of Geophysical Research: Atmospheres 105, no. D6 (2000): 7337-7356.

Santer, Benjamin David, T. M. L. Wigley, C. Doutriaux, J. S. Boyle, J. E. Hansen, P. D. Jones, G. A. Meehl, E. Roeckner, S. Sengupta, and K. E. Taylor. "Accounting for the effects of volcanoes and ENSO in comparisons of modeled and observed temperature trends." Journal of Geophysical Research: Atmospheres 106, no. D22 (2001): 2803328059. 
Sarachik, E. S., and D. J. Vimont. "Decadal variability in the Pacific." Chaos in geophysical flows (2003): 125-167.

Schlesinger, Michael E., and Navin Ramankutty. "An oscillation in the global climate system of period 65-70 years." Nature 367, no. 6465 (1994): 723-726.

Schneider, Niklas, Arthur J. Miller, and David W. Pierce. "Anatomy of North Pacific decadal variability." Journal of climate 15, no. 6 (2002): 586-605.

Schurer, Andrew P., Gabriele C. Hegerl, and Stephen P. Obrochta. "Determining the likelihood of pauses and surges in global warming." Geophysical Research Letters 42, no. 14 (2015): 5974-5982.

Seager, Richard, Naomi Naik, and Laura Vogel. "Does global warming cause intensified interannual hydroclimate variability?." Journal of Climate 25, no. 9 (2012): 3355-3372.

Seidel, D.J., Fu, Q., Randel, W.J. and Reichler, T.J., 2008. Widening of the tropical belt in a changing climate. Nature geoscience, 1(1), p.21.

Servain, Jacques, Ilana Wainer, Julian P. McCreary, and Alain Dessier. "Relationship between the equatorial and meridional modes of climatic variability in the tropical Atlantic." Geophysical Research Letters 26, no. 4 (1999): 485-488.

Sheinbaum, Julio. "Current theories on El Nino-southern oscillation: a review." GEOFISICA INTERNACIONAL-MEXICO- 42, no. 3 (2003): 291-306.

Singarayer, Joy S., Jonathan L. Bamber, and Paul J. Valdes. "Twenty-first-century climate impacts from a declining Arctic sea ice cover." Journal of Climate 19, no. 7 (2006): 1109-1125.

Smith, Thomas M., Richard W. Reynolds, Robert E. Livezey, and Diane C. Stokes. "Reconstruction of historical sea surface temperatures using empirical orthogonal functions." Journal of Climate 9, no. 6 (1996): 1403-1420.

Smith, T.M., R.W. Reynolds, T.C. Peterson, and J. Lawrimore, 2008: Improvements NOAAs Historical Merged Land-Ocean Temp Analysis (1880-2006). Journal of Climate, 21, 2283-2296.

Smith, Karen L., Lorenzo M. Polvani, and Daniel R. Marsh. "Mitigation of 21st century Antarctic sea ice loss by stratospheric ozone recovery." Geophysical Research Letters39, no. 20 (2012). 
Smith, Doug M., Adam A. Scaife, George J. Boer, Mihaela Caian, Francisco J. DoblasReyes, Virginie Guemas, Ed Hawkins et al. "Real-time multi-model decadal climate predictions." Climate dynamics 41, no. 11-12 (2013): 2875-2888.

Soden, Brian J., Richard T. Wetherald, Georgiy L. Stenchikov, and Alan Robock. "Global cooling after the eruption of Mount Pinatubo: A test of climate feedback by water vapor." science296, no. 5568 (2002): 727-730.

Soden, Brian J., and Isaac M. Held. "An assessment of climate feedbacks in coupled ocean-atmosphere models." Journal of Climate 19, no. 14 (2006): 3354-3360.

Solomon, S., Rosenlof, K. H., Portmann, R. W., Daniel, J. S., Davis, S. M., Sanford, T. J., \& Plattner, G. K. (2010). Contributions of stratospheric water vapor to decadal changes in the rate of global warming. Science, 327(5970), 1219-1223.

Solomon, Susan, John S. Daniel, R. R. Neely, J-P. Vernier, Ellsworth G. Dutton, and Larry W. Thomason. "The persistently variable "background" stratospheric aerosol layer and global climate change." Science 333, no. 6044 (2011): 866-870.

Steinman, Byron A., Michael E. Mann, and Sonya K. Miller. "Atlantic and Pacific multidecadal oscillations and Northern Hemisphere temperatures." Science 347, no. 6225 (2015): 988-991.

Stevenson, S. L. "Significant changes to ENSO strength and impacts in the twenty-first century: Results from CMIP5." Geophysical Research Letters 39, no. 17 (2012).

Stowe, L. L., R. M. Carey, and P. P. Pellegrino. "Monitoring the Mt. Pinatubo aerosol layer with NOAA/11 AVHRR data." Geophysical research letters 19, no. 2 (1992): 159162.

Stroeve, Julienne, Marika M. Holland, Walt Meier, Ted Scambos, and Mark Serreze. "Arctic sea ice decline: Faster than forecast." Geophysical research letters 34, no. 9 (2007).

Taylor, Karl E. "Summarizing multiple aspects of model performance in a single diagram." Journal of Geophysical Research: Atmospheres 106, no. D7 (2001): 71837192.

Taylor, Karl E., Ronald J. Stouffer, and Gerald A. Meehl. "An overview of CMIP5 and the experiment design." Bulletin of the American Meteorological Society 93, no. 4 (2012): 485.

Tebaldi, Claudia, and Reto Knutti. "The use of the multi-model ensemble in probabilistic climate projections." Philosophical Transactions of the Royal Society of London A: Mathematical, Physical and Engineering Sciences 365, no. 1857 (2007): 2053-2075. 
Tebaldi, Claudia, Julie M. Arblaster, and Reto Knutti. "Mapping model agreement on future climate projections." Geophysical Research Letters 38, no. 23 (2011).

Thomson, Allison M., Katherine V. Calvin, Steven J. Smith, G. Page Kyle, April Volke, Pralit Patel, Sabrina Delgado-Arias et al. "RCP4. 5: a pathway for stabilization of radiative forcing by 2100." Climatic change 109, no. 1-2 (2011): 77.

Timmermann, Axel, Josef Oberhuber, Andreas Bacher, Monika Esch, Mojib Latif, and Erich Roeckner. "Increased El Niño frequency in a climate model forced by future greenhouse warming." Nature 398, no. 6729 (1999): 694-697.

Tokinaga, Hiroki, and Shang-Ping Xie. "Weakening of the equatorial Atlantic cold tongue over the past six decades." Nature Geoscience 4, no. 4 (2011): 222-226.

Trenberth, Kevin E., Julie M. Caron, David P. Stepaniak, and Steve Worley. "Evolution of El Niño-Southern Oscillation and global atmospheric surface temperatures." Journal of Geophysical Research: Atmospheres 107, no. D8 (2002).

Trenberth, K.E., 2009: An imperative for adapting to climate change: Tracking Earth's global energy. Current Opinion in Environmental Sustainability, 1, 19-27. DOI 10.1016/j.cosust.2009.06.001.

Trenberth, K.E., and J.T. Fasullo (2013), An apparent hiatus in global warming? Earth's Future, 1, 19-32, doi: 10.1002/2013EF000165

Trenberth, Kevin E. "Has there been a hiatus." Science 349, no. 6249 (2015): 691.

Turner, John, Josefino C. Comiso, Gareth J. Marshall, Tom A. Lachlan-Cope, Tom Bracegirdle, Ted Maksym, Michael P. Meredith, Zhaomin Wang, and Andrew Orr. "Nonannular atmospheric circulation change induced by stratospheric ozone depletion and its role in the recent increase of Antarctic sea ice extent." Geophysical Research Letters 36, no. 8 (2009).

van Oldenborgh, Geert Jan, Francisco J. Doblas-Reyes, Bert Wouters, and Wilco Hazeleger. "Decadal prediction skill in a multi-model ensemble." Climate dynamics 38, no. 7-8 (2012): 1263-1280.

Van Vuuren, Detlef P., Jae Edmonds, Mikiko Kainuma, Keywan Riahi, Allison Thomson, Kathy Hibbard, George C. Hurtt et al. "The representative concentration pathways: an overview." Climatic change 109, no. 1-2 (2011): 5.

Van Vuuren, Detlef P., Elke Stehfest, Michel GJ den Elzen, Tom Kram, Jasper van Vliet, Sebastiaan Deetman, Morna Isaac et al. "RCP2. 6: exploring the possibility to keep global mean temperature increase below 2 C." Climatic Change 109, no. 1-2 (2011): 95. 
Vecchi, Gabriel A., Brian J. Soden, Andrew T. Wittenberg, Isaac M. Held, Ants Leetmaa, and Matthew J. Harrison. "Weakening of tropical Pacific atmospheric circulation due to anthropogenic forcing." Nature 441, no. 7089 (2006): 73-76.

Vecchi, Gabriel A., and Brian J. Soden. "Global warming and the weakening of the tropical circulation." Journal of Climate 20, no. 17 (2007): 4316-4340.

Vecchi, Gabriel A., Amy Clement, and Brian J. Soden. "Examining the tropical Pacific's response to global warming." Eos, Transactions American Geophysical Union 89, no. 9 (2008): 81-83.

von Storch, Hans, and Francis W. Zwiers. "Statistical analysis in climate research." (2002).

Vyushin, D. I., P. J. Kushner, and Francis Zwiers. "Modeling and understanding persistence of climate variability." Journal of Geophysical Research: Atmospheres 117, no. D21 (2012).

Wang, Muyin, and James E. Overland. "A sea ice free summer Arctic within 30 years?." Geophysical Research Letters 36, no. 7 (2009).

Wang, Chunzai, Clara Deser, Jin-Yi Yu, Pedro DiNezio, and Amy Clement. "El Nino and southern oscillation (ENSO): a review." Coral Reefs of the Eastern Pacific (2012): 3-19.

Wang, Chunzai, Liping Zhang, Sang-Ki Lee, Lixin Wu, and Carlos R. Mechoso. "A global perspective on CMIP5 climate model biases." Nature Climate Change 4, no. 3 (2014): 201-205.

Watanabe, Masahiro, Hideo Shiogama, Hiroaki Tatebe, Michiya Hayashi, Masayoshi Ishii, and Masahide Kimoto. "Contribution of natural decadal variability to global warming acceleration and hiatus." Nature Climate Change 4, no. 10 (2014): 893.

Webster, Peter J., Andrew M. Moore, Johannes P. Loschnigg, and Robert R. Leben. "Coupled ocean-atmosphere dynamics in the Indian Ocean during 1997-98." Nature 401, no. 6751 (1999): 356-360.

Wang, Muyin, and James E. Overland. "A sea ice free summer Arctic within 30 years?." Geophysical Research Letters 36, no. 7 (2009).

Weigel, Andreas P., M. A. Liniger, and C. Appenzeller. "Can multi-model combination really enhance the prediction skill of probabilistic ensemble forecasts?." Quarterly Journal of the Royal Meteorological Society 134, no. 630 (2008): 241-260. 
Weisheimer, A., F. J. Doblas-Reyes, T. N. Palmer, A. Alessandri, A. Arribas, M. Déqué, Noel Keenlyside, M. MacVean, A. Navarra, and P. Rogel. "ENSEMBLES: A new multimodel ensemble for seasonal-to-annual predictions-Skill and progress beyond DEMETER in forecasting tropical Pacific SSTs." Geophysical research letters 36, no. 21 (2009).

White, Warren B., and Ray G. Peterson. "An Antarctic circumpolar wave in surface pressure, wind, temperature and sea-ice extent." Nature 380, no. 6576 (1996): 699.

Wigley, T. M. L. "ENSO, volcanoes and record-breaking temperatures." Geophysical Research Letters 27, no. 24 (2000): 4101-4104.

Wild, Martin, Doris Folini, Christoph Schär, Norman Loeb, Ellsworth G. Dutton, and Gert König-Langlo. "The global energy balance from a surface perspective." Climate dynamics40, no. 11-12 (2013): 3107-3134.

Wilks, Daniel S. Statistical methods in the atmospheric sciences. Vol. 100. Academic press, 2011.

Wu, L., Z. Liu, R. Gallimore, R. Jacob, D. Lee, and Y. Zhong. "Pacific Decadal Variability: The Tropical Pacific Mode and the North Pacific Mode*." Journal of Climate 16, no. 8 (2003): 1101-1120.

Wu, Zhaohua, Norden E. Huang, Steven R. Long, and Chung-Kang Peng. "On the trend, detrending, and variability of nonlinear and nonstationary time series." Proceedings of the National Academy of Sciences 104, no. 38 (2007): 14889-14894.

Xie, Shang-Ping, and James A. Carton. "Tropical Atlantic variability: Patterns, mechanisms, and impacts." Earth's Climate (2004): 121-142.

Yamagata, Toshio, Swadhin K. Behera, Jing-Jia Luo, Sebastien Masson, Mark R. Jury, and Suryachandra A. Rao. "Coupled ocean-atmosphere variability in the tropical Indian Ocean." Earth's Climate (2004): 189-211.

Yeh, Sang-Wook, and Ben P. Kirtman. "Tropical Pacific decadal variability and ENSO amplitude modulation in a CGCM." Journal of Geophysical Research: Oceans (19782012) 109, no. C11 (2004).

Yeh, Sang-Wook, and Ben P. Kirtman. "Pacific decadal variability and decadal ENSO amplitude modulation." Geophysical research letters 32, no. 5 (2005).

Yeh, Sang-Wook, and Ben P. Kirtman. "ENSO amplitude changes due to climate change projections in different coupled models." Journal of climate 20, no. 2 (2007): 203-217. 
Yun, W. T., Lydia Stefanova, and T. N. Krishnamurti. "Improvement of the multimodel superensemble technique for seasonal forecasts." Journal of Climate 16, no. 22 (2003): 3834-3840.

Zebiak, Stephen E., and Mark A. Cane. "A Model El Niño-Southern Oscillation." Monthly Weather Review 115, no. 10 (1987): 2262-2278.

Zebiak, Stephen E. "Air-sea interaction in the equatorial Atlantic region." Journal of Climate 6, no. 8 (1993): 1567-1586.

Zhang, Rong, and Thomas L. Delworth. "Impact of Atlantic multidecadal oscillations on India/Sahel rainfall and Atlantic hurricanes." Geophysical Research Letters 33, no. 17 (2006).

Zhang, Minghua, and Hua Song. "Evidence of deceleration of atmospheric vertical overturning circulation over the tropical Pacific." Geophysical research letters 33, no. 12 (2006).

Zhang, Xiangdong, and John E. Walsh. "Toward a seasonally ice-covered Arctic Ocean: Scenarios from the IPCC AR4 model simulations." Journal of Climate 19, no. 9 (2006): 1730-1747.

Zhang, Rong, and Thomas L. Delworth. "Impact of the Atlantic multidecadal oscillation on North Pacific climate variability." Geophysical Research Letters 34, no. 23 (2007).

Zhang, Jinlun. "Increasing Antarctic sea ice under warming atmospheric and oceanic conditions." Journal of Climate 20, no. 11 (2007): 2515-2529.

Zheng, Xiao-Tong, Shang-Ping Xie, Gabriel A. Vecchi, Qinyu Liu, and Jan Hafner. "Indian Ocean dipole response to global warming: Analysis of ocean-atmospheric feedbacks in a coupled model." Journal of Climate 23, no. 5 (2010): 1240-1253.

Zinke, Jens, W-Chr Dullo, G. A. Heiss, and Anton Eisenhauer. "ENSO and Indian Ocean subtropical dipole variability is recorded in a coral record off southwest Madagascar for the period 1659 to 1995." Earth and Planetary Science Letters 228, no. 1 (2004): 177194.

Zwally, H. Jay, Josefino C. Comiso, Claire L. Parkinson, Donald J. Cavalieri, and Per Gloersen. "Variability of Antarctic sea ice 1979-1998." Journal of Geophysical Research: Oceans 107, no. C5 (2002). 
VITA

HEATHER VAZQUEZ

Born, Gainesville, FL, USA

2009-2012

B.S., Geoscience with Atmospheric Track

Florida International University

Miami, Florida

2013

Graduate Teaching Assistant

Florida International University

Miami, Florida

Second Place Student Poster Presentation, 93 ${ }^{\text {rd }}$ American Meteorological Society Annual Meeting

2015

Best Ph.D. Oral presentation, runner up, Earth and Environment Graduate Research Symposium, Florida International University

2016

M.S., Geoscience with Atmospheric Track

Florida International University

Miami, Florida

Doctoral Candidate

Florida International University

Miami, Florida

2017

Best Ph.D. Oral presentation, Earth and Environment Graduate Research Symposium, Florida International University

\section{PUBLICATIONS AND PRESENTATIONS}

Burgman, R. J., B. P. Kirtman, A. C. Clement, and H. Vazquez, (2017), Model evidence for low-level cloud feedback driving persistent changes in atmospheric circulation and regional hydroclimate. Geophysical Research Letters, 44(1), 428-437.

Vazquez, H., R.J. Burgman. Evaluating Changes of Natural Variability in the Oceans on a Warmer Globe in CMIP5 Models. Earth and Environment Graduate Student Symposium, Miami, FL February 16, 2018 
Vazquez, H., R.J. Burgman. Evaluating Changes of Natural Variability in the Oceans on a Warmer Globe in CMIP5 Models. 98 ${ }^{\text {th }}$ American Meteorological Society Annual Meeting, Austin, TX, January 8-11, 2018

Vazquez, H., R.J. Burgman. Evaluating Changes of Natural Variability in the Oceans on a Warmer Globe in CMIP5 Models. American Geophysical Union, New Orleans, LA, December 11-15, 2017

Vazquez, H., R.J. Burgman. The role of Natural Variability in Climate Change. Florida International University, Earth and Environment Graduate Student Symposium, Miami, FL February 24, 2017

Vazquez, H., R.J. Burgman. The role of Natural Variability in Climate Change. Florida International University, Earth and Environment Graduate Student Symposium, Miami, FL February 20, 2015

Vazquez, H., J. Cangialosi, J. Estupiñán. An examination of the National Hurricane Center track forecasts of tropical cyclones over Florida and the Gulf of Mexico. Poster Presentation: $41^{\text {st }}$ Conference on Hurricane and Tropical Meteorology, San Diego, CA, March 30 - April 4, 2014

Vazquez, H., R. Schumacher. Variability of Heavy Precipitation by Long-Lived Mesoscale Convective Vortices found in the Southern Plains. Poster Presentation: 93 ${ }^{\text {rd }}$ American Meteorological Society Annual Meeting, Austin, TX, January 6-10, 2013 